\title{
Chapter 8 \\ Systematic Survey of Zoonotic and Sapronotic Microbial Agents
}

Viruses, bacteria, fungi, protozoa, and metazoa (parasitic worms and arthropods) are among the aetiological agents of zoonoses and sapronoses. Our survey only concerns the agents of microbial diseases (i.e., not the metazoan invasions and infestations), and is arranged according to systematic position. In addition, a new zoonotic disease caused by prions - variant of Creutzfeld-Jakob disease - is included.

Sapronotic agents are marked with an asterisk ${ }^{*}$, and those agents the source of which can be simultaneously both animals and extraanimal substrates (i.e., the agents of zoonoses plus sapronoses), are marked with an asterisk in parentheses $\left(^{*}\right)$. Occasionally also those anthroponotic agents are mentioned, biological vectors of which are haematophagous arthropods - those are labelled with ${ }^{* *}$, while $\left.{ }^{* *}\right)$ marks the anthroponotic agents which can occasionally also cause a zoonosis under certain circumstance (e.g., avian influenza A virus).

In the paragraph named "Bio-containment", the grades of Biosafety Level (BSL) practices and containment are given, which are related to laboratory work with the particular agent (Anonymous 2007). The scores mean, in short:

BSL-1 - the agent does not usually cause any illness, and standard microbiological practices are required (work on open bench);

BSL-2 - the agent can cause an illness and care is required to control aerosols and contamination (class I or II biosafety cabinets [BSC] are required for aerosol producing procedures; limited access);

BSL-3 - the agent causes a serious illness and it can spread aerogenically; class II BSC required for all manipulations with infectious material; restricted access, air lock facility, controlled uni-directional air flow, exhaust air discharged away from building (with certain viruses via HEPA filtration);

BSL-4 - the agent causes serious, life-threatening disease and it can spread aerogenically, there is no known effective therapy nor vaccination against it; the work with such agent necessitates rigorous containment of all manipulations, change of clothing and shower; class II BSC is adequate only when all laboratory personnel are immunized or insusceptible to the agent, otherwise 
class III BSC or hermetically closed positive pressure suits are required; the facility must be equivalent to a separate building with HEPA filtration of all exhaust air, and provided with a double-door autoclave.

For dangerous agents of the bio-containment grade BSL-3, the BSL-2 practices and facilities can be generally used for the less risky nonpropagative laboratory procedures such as serology with inactivated antigens or for staining of fixed (inactivated) impression smears, while BSL-3 practices and facilities have to be used for propagative techniques such as inoculation (and harvesting) of cell cultures, embryonated eggs or experimental animals, the necropsy of infected animals and manipulations with infected tissues. A more detailed information on BSL and risk groups of microbes is given below (CDC 2007).

Classification of infectious microorganisms by risk group

\begin{tabular}{|c|c|c|}
\hline $\begin{array}{l}\text { Risk group } \\
\text { classification }\end{array}$ & $\begin{array}{l}\text { NIH guidelines for research involving } \\
\text { recombinant DNA molecules } 2002\end{array}$ & $\begin{array}{l}\text { World Health Organization laboratory } \\
\text { biosafety manual 3rd edition } 2004\end{array}$ \\
\hline Risk group 1 & $\begin{array}{l}\text { Agents that are not associated with } \\
\text { disease in healthy adult humans }\end{array}$ & $\begin{array}{l}\text { (No or low individual and community } \\
\text { risk) } \\
\text { A microorganism that is unlikely to } \\
\text { cause human or animal disease }\end{array}$ \\
\hline Risk group 2 & $\begin{array}{l}\text { Agents that are associated with } \\
\text { human disease which is rarely } \\
\text { serious and for which preventive or } \\
\text { therapeutic interventions are often } \\
\text { available }\end{array}$ & $\begin{array}{l}\text { (Moderate individual risk; low } \\
\text { community risk). A pathogen that } \\
\text { can cause human or animal disease } \\
\text { but is unlikely to be a serious } \\
\text { hazard to laboratory workers, the } \\
\text { community, livestock or the } \\
\text { environment. Laboratory exposures } \\
\text { may cause serious infection, but } \\
\text { effective treatment and preventive } \\
\text { measures are available and the risk } \\
\text { of spread of infection is limited }\end{array}$ \\
\hline Risk group 3 & $\begin{array}{l}\text { Agents that are associated with } \\
\text { serious or lethal human disease for } \\
\text { which preventive or therapeutic } \\
\text { interventions may be available } \\
\text { (high individual risk but low } \\
\text { community risk) }\end{array}$ & $\begin{array}{l}\text { (High individual risk; low community } \\
\text { risk). A pathogen that usually } \\
\text { causes serious human or animal } \\
\text { disease but does not ordinarily } \\
\text { spread from one infected individual } \\
\text { to another. Effective treatment and } \\
\text { preventive measures are available }\end{array}$ \\
\hline Risk group 4 & $\begin{array}{l}\text { Agents that are likely to cause serious } \\
\text { or lethal human disease for which } \\
\text { preventive or therapeutic } \\
\text { interventions are not usually } \\
\text { available (high individual risk and } \\
\text { high community risk) }\end{array}$ & $\begin{array}{l}\text { A pathogen that usually causes } \\
\text { serious human or animal disease } \\
\text { and that can be readily transmitted } \\
\text { from one individual to another, } \\
\text { directly or indirectly. Effective } \\
\text { treatment and preventive measures } \\
\text { are not usually available }\end{array}$ \\
\hline
\end{tabular}


Biosafety Level 1 is suitable for work involving well-characterized agents not known to consistently cause disease in immunocompetent adult humans, and present minimal potential hazard to laboratory personnel and the environment. BSL-1 laboratories are not necessarily separated from the general traffic patterns in the building. Work is typically conducted on open bench tops using standard microbiological practices. Special containment equipment or facility design is not required, but may be used as determined by appropriate risk assessment. Laboratory personnel must have specific training in the procedures conducted in the laboratory and must be supervised by a scientist with training in microbiology or a related science.

Biosafety Level 2 is suitable for work involving agents that pose moderate hazards to personnel and the environment. It differs from BSL-1 in that (1) laboratory personnel have specific training in handling pathogenic agents and are supervised by scientists competent in handling infectious agents and associated procedures; (2) access to the laboratory is restricted when work is being conducted; and (3) all procedures in which infectious aerosols or splashes may be created are conducted in BSCs or other physical containment equipment.

Biosafety Level 3 is applicable to clinical, diagnostic, teaching, research, or production facilities where work is performed with indigenous or exotic agents that may cause serious or potentially lethal disease through inhalation route exposure. Laboratory personnel must receive specific training in handling pathogenic and potentially lethal agents, and must be supervised by scientists competent in handling infectious agents and associated procedures. All procedures involving the manipulation of infectious materials must be conducted within BSCs (preferably Class II or Class III), or other physical containment devices, other physical containment devices, or by personnel wearing appropriate personal protective equipment. The laboratory must be separated from areas that are open to unrestricted traffic flow within the building.

Biosafety Level 4 is required for work with dangerous and exotic agents that pose a high individual risk of life-threatening disease, aerosol transmission, or related agent with unknown risk of transmission. Agents with a close or identical antigenic relationship to agents requiring BSL-4 containment must be handled at this level until sufficient data are obtained either to confirm continued work at this level, or re-designate the level. Laboratory staff must have specific and thorough training in handling extremely hazardous infectious agents. Laboratory staff must understand the primary and secondary containment functions of standard and special practices, containment equipment, and laboratory design characteristics. All laboratory staff and supervisors must be competent in handling agents and procedures requiring BSL-4 containment. Access to the laboratory is controlled by the laboratory supervisor in accordance with institutional policies. There are two models for BSL-4 laboratories: 
(1) A Cabinet Laboratory where all handling of agents must be performed in a Class III BSC.

(2) A Suit Laboratory where personnel must wear a positive pressure protective suit.

BSL-4 Cabinet and Suit Laboratories have special engineering and design features to prevent microorganisms from being disseminated into the environment. The BSL-4 cabinet laboratory consists of either a separate building or a clearly demarcated and isolated zone within a building. For BSL-4 containment agents, the BSL-3 laboratory procedures can be sometimes used when all personnel is effectively vaccinated against the disease and stringent laboratory protocols are applied (example: TBE virus).

\subsection{Prions}

Since 1986, an explosive epizootic of bovine spongiform encephalopathy (BSE) has occurred in the UK: about 166,000 cattle were affected up to 1997, with peak incidence between 1992 and 1993. According to the main symptom - loss of neuromuscular coordination - the syndrome has informally been called "mad cow disease". It originated from the use of ovine meat-bone meal added to the fodder of cattle; some of the source sheep cadavers had been infected with scrapie, another spongiform encephalopathy, known and described in Great Britain since 1732. Scrapie was widely dispersed in Britain by the mass use of a vaccine against louping ill (1938 and later) unintentionally contaminated with scrapie. In cattle a disease similar to scrapie was never observed before 1986; in the 1980s, scrapie obviously overcame the species barrier and adapted to cattle (a "host-jumping event").

The infectious agent in BSE and in similar "slow" or "unconventional" diseases, called generally transmissive spongiform encephalopathies (TSE), with an incubation period usually over 3 years in diverse mammals (mink, cat, deer) including man (Creutzfeld-Jakob disease, or kuru) are so-called prions (Stanley B. Prusiner 1982: "proteinaceous infectious particles", a modified acronym), causing vacuolisation of the brain tissue that looks like a sea sponge or an Emmental cheese. It is interesting that the prion $\mathrm{PrP}^{\mathrm{c}}$ is a physiological normal protein in the $\mathrm{CNS}$, which however can change its conformation (spatial configuration) from the molecule with a prevailing $\alpha$-helix structure to the pathogenic $\beta$-folded flat structure $\mathrm{PrP}^{\mathrm{Sc}}$ (the index Sc means scrapie). Following this structural rearrangement, the molecule becomes hydrophobic and resistant to disintegration. An accumulation of these $\mathrm{PrP}^{\mathrm{Sc}}$ prions in the $\mathrm{CNS}$ leads to the subsequent destruction of neurons. The pathological isoform of the prion posseses several other quite unusual characteristics: it is non-antigenic (no humoral antibodies are produced against the prion), resistant against proteases (this is why it accumulates in tissue not being metabolised), high temperature (it does not inactivate at $121^{\circ} \mathrm{C}$ for $60 \mathrm{~min}$ nor at $240^{\circ} \mathrm{C}$ for $1 \mathrm{~min}$ ) and common disinfection liquids (peracetic acid, phenol, alcohols, formalin, ultraviolet and microvave irradiation), while undiluted sodium hypochlorite, $4 \% \mathrm{NaOH}$, or autoclaving at $134^{\circ} \mathrm{C}$ for $60 \mathrm{~min}$ are effective. 


\subsubsection{Prion vCJD}

Creutzfeld-Jakob disease (CJD) was described in 1920-1921. It is a rare, but absolutely fatal disease with a mean incidence of about 0.4 cases per 10 million population, usually hereditary but there is also a transmissible form e.g. during corneal transplantation or on application of hypophyseal growth hormone. A new variant of human CJD (abbreviated as "vCJD") has been observed in Britain since 1994, differing from classic CJD by a substantially shorter incubation period (months rather than years), a much higher incidence rate (the original inter-annual increase of the incidence was 20-30\%) and in also affecting young people. This vCJD was detected disproportionately more often in families of farmers in areas where BSE was endemic and after contact with diseased cattle. There is a justified hypothesis that vCJD is, in fact, BSE transmitted to humans, it means a zoonosis. This hypothesis has been promoted by results of several experiments with BSE on animals (monkeys and mice). The CNS, bone marrow, neural ganglions, ileum, retina, and so-called mechanically-recovered meat (remnants of meat detached from backbone and ribs, used for preparation of hamburgers, sausages and minced meat in tins for humans and pet animals) are regarded as risky bovine tissues for consumption. Until August 2010, "only" 212 people had died of vCJD (against the originally expected many hundreds or thousands of victims), out of which 43 patients were from outside Great Britain (France 25 cases, Ireland 6, the Netherlands 2, Spain 1, Portugal 1, Italy 2, Canada 1, USA 3, Japan 1, and Saudi Arabia 1). The epidemic was efficiently controlled by drastic veterinary measures: culling, and the ban on feeding meat-bone meal to cattle.

Bio-containment: BSL-2.

Diagnosis of transmissive spongiform encephalopathies is carried out using electroencephalography, CT, histopathology, immunohistochemistry, molecular biology (PCR), ELISA and WB.

\subsection{Viruses}

Most zoonotic viruses have an RNA genome, although some zoonoses are also caused by DNA herpetic and pox viruses. Numerically most common viral agents of zoonoses are arboviruses. The acronym arbovirus (for "arthropod-borne virus", coined by W. M. Hammon in 1958) was suggested by W. C. Reeves, and recommended for general use by the WHO in 1960. Arboviruses do not constitute a taxonomic unit, but an ecological group with a common mode of transmission to vertebrates. Their existence in nature is conditioned by replication in blood-feeding (haematophagous) arthropods and by an interaction between these arthropods and vertebrates. It is important that transmission from the vector arthropod to the recipient vertebrate is biological, not mechanical. The ability of replication in two phylogenetically and physiologically diverse organisms - poikilothermic arthropods 
and homeothermic vertebrates - and, at the same time, at largely varying ambient temperatures, is a remarkable characteristic and one that is in other viruses unusual.

For the transmission of arboviruses by competent arthropod vectors the following conditions must be fulfilled:

(1) the vector is infected by feeding on a viraemic vertebrate host or during cofeeding with infected vectors,

(2) the infectious dose must pass to the vector's gut lumen,

(3) the virions are adsorbed on the membrane of epithelial gut cells, penetrate into them by endocytosis or fusion of the virion envelope with the cell membrane, and replicate intracellularly,

(4) infectious virions disseminate from epithelial gut cells in the haemocel of the vector,

(5) they further migrate into salivary glands,

(6) the virions are transmitted in saliva by feeding of the vector on a susceptible host.

Nearly 500 arboviruses are registered (International Catalogue of Arboviruses) at present. They belong to 9 families: Bunyaviridae (52\% of all arboviruses), Reoviridae (17\%), Flaviviridae (12\%), Rhabdoviridae (10\%), Togaviridae (6\%), Orthomyxoviridae, Poxviridae, Asfarviridae and Nodaviridae (the latter four all less than $1 \%$ ). However, only about 100 arboviruses have been reported as pathogenic to humans.

The diagnosis of arbovirus and other virus infections is largely based on serological laboratory analysis, optimally by using paired blood serum samples taken at an interval of 2-3 weeks. Recent infection is said to be demonstrated when the patient seroconverts between the two intervals (i.e., the 1st sample is without antibodies, while the 2 nd sample contains antibodies to a particular virus) or when there is an at least fourfold increase of antibody titre against the virus, as revealed in different serological tests, e.g. ELISA, HIT, CFT, VNT, IFA etc. (Lennette, Schmidt et al. 1974). If there is only one (convalescent) serum sample of the patient available, the differentiation of recent infection from a past one can be done by testing for IgM and IgG immunoglobulins - in recent infections IgM antibodies prevail over IgG. A very significant, but difficult and time-consuming technique is the isolation of the virus agent from the blood, CSF or bioptic samples of the patient using inoculation of suckling mice, cell cultures or chicken embryos. In the last decade, isolation techniques have started to be intensively replaced by molecular techniques detecting specific nucleic acids of particular viral agents: traditional or real-time PCR and RT-PCR, nested (RT-)PCR, RLB, sequencing, and others. These methods are excellent tools, although they also have some specific limitations (e.g., they do not show whether the nucleic acid fragments originate from a viable virus). Various immunohistochemistry techniques are also very convenient for the detection of viruses in the vertebrate tissues, and usually they are highly specific.

Specific therapy for viral diseases usually does not exist, and therefore symptomatic treatment, rest in bed, a sufficient supply of fluids, infusions and antipyretics 
are recommended; in certain cases antiserum (a specific immunoglobulin) may help, when applied immediately after infection. Only a few virus diseases can be treated with antivirals such as nucleotide analogues, e.g. ribavirin in RNA virus infections, or acyclovir in some DNA viruses. The optimum specific measure against virus infection is vaccination. Unfortunately, very few vaccines are available against zoonotic virus diseases at present: YF, JE, TBE, WEE/EEE/VEE, RVF, and lyssa.

\subsubsection{Family Togaviridae}

\section{Alphaviruses EEE, WEE, VEE (American Equine Encephalomyelitides)}

Virions of the genus Alphavirus are spherical (60-70 nm), enveloped, contain one molecule of ss(+)RNA sized 10-12 kbp, and 2-3 surface glycoproteins E1, E2 (E3). Alphaviruses occurring in New and Old World differentiated phylogenetically some 2-3 thousands of years. The first virus of this group (WEE) was isolated from a dead horse in 1933.

Source of infection (natural host range): birds (EEE, WEE: house sparrow, house finch, and others; less in VEE), horse, rodents (VEE and EEE: Peromyscus, Oryzomys, Proechimys; WEE: Spermophilus, Sciurus, Microtus), leporids (WEE: Lepus californicus), marsupials (VEE, EEE), bats (VEE), snakes and frogs (VEE, WEE). Migratory birds can transport EEE virus.

Animal disease: encephalomyelitis in horses (EEE, WEE, VEE), some other mammals, and in pheasants (EEE).

Transmission mode (Figs. 8.1, 8.2): culicine mosquitoes, less other arthropods. Main cycles in nature: sylvatic (endemic) and urban (epidemic, synanthropic): WEE - Culex tarsalis and Ae. melanimon (but also Cx. pipiens, Cx. quinquefasciatus, Cx. restuans, Ae. dorsalis, Ae. nigromaculis, Culiseta inornata, An. freeborni as secondary or occasional vectors) + birds as vertebrate hosts (house sparrows in the urban epidemic cycle); Ae. melanimon acts as a bridge vector of WEE virus $\rightarrow$ horse and man; reptiles may also host the virus (virus overwintering in them is possible). In EEE, the main vector is Culiseta melanura (also Cx. salinarius, Ae. mitchellae, An. crucians, Cx. restuans as secondary vectors) + birds as vertebrate hosts; Ae. sollicitans, Ae. vexans, and Coquillettidia perturbans act as bridge vectors of EEE $\rightarrow$ man and horse. VEE (including the enzootic subtypes Mucambo, Tonate, Everglades, and Pixuna) is vectored by Ae. taeniorhynchus (primary vector), Psorophora columbiae, P. discolor, Culex spp., An. crucians, Coquillettidia perturbans, Mansonia titillans (Coquillettidia and Aedes spp. in epidemic cycle) + rodents. Congenital infections have also been described (foetal abnormalities, abortion).

Human disease: EEE, WEE, VEE - fever (also chills, headache, body aches, lethargy, nausea, vomiting, prostration, inflammation of throat, cervical 
Fig. 8.1 Natural cycle of WEE and EEE viruses $(C x$. tarsalis is the principal vector of WEE, while Cs. melanura in EEE) (drawing by Ivo Rudolf)
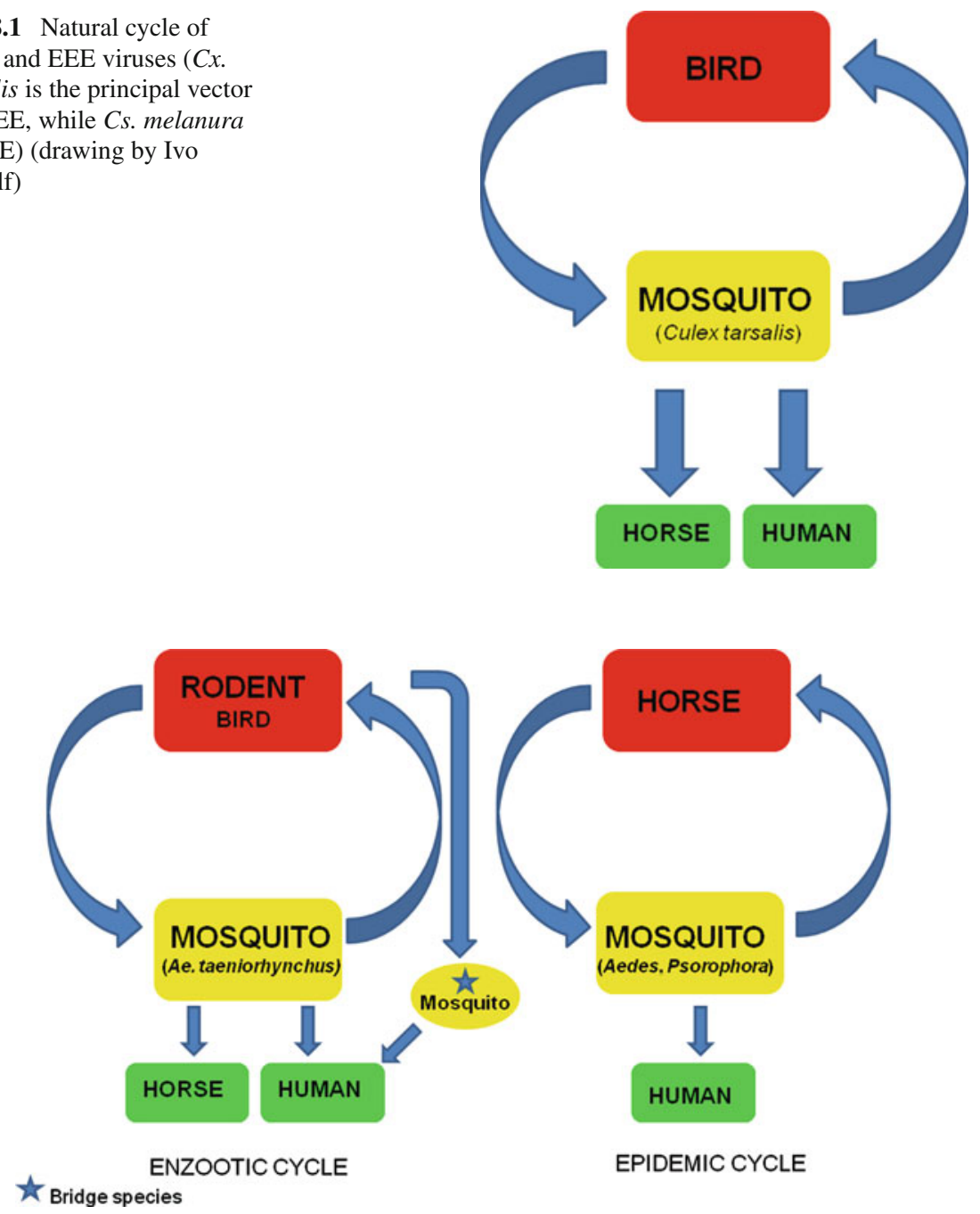

Fig. 8.2 The cycles of VEE virus (drawing by Ivo Rudolf)

lymphadenitis, dizziness, encephalitis). The highest fatality rate has been observed in EEE (35-75\%, against about 20\% in WEE), and persistent sequelae (paralysis, pareses) are comparatively frequent, especially in children - some of them may remain mentally deficient. A total of 221 humans cases of EEE were confirmed in the USA in 1964-2005. South-American strains of EEE virus are obviously less virulent, only 2 fatal cases have been reported. WEE epidemics: 1941 USA (3,000 human cases); 1964-1997, USA a total 639 cases. A major outbreak of VEE occurred in Venezuela and Columbia in 1962-1964 when about 30,000 persons were ill 
and 300 of them died, a smaller one in Mexico and Texas in 1971 (84 cases), and a huge one in 1995 in Columbia and Venezuela with 75,000-100,000 cases.

Bio-containment: BSL-2 (WEE, EEE), BSL-3 (VEE: a high risk of laboratory infections).

Prevention: there are mono-, bi- and tri-valent inactivated vaccines (horse, human), their use is however limited (usually applicable for laboratory personnel).

Geographical distribution: North, Central and South America. However, only the less virulent subtypes of VEE occur in North America (Tonate, Everglades). Rio Negro and Pixuna viruses are other subtypes of VEE, occurring in Argentina and Brazil, respectively. Brand new data indicate that EEE virus strains that circulate in Central and South America might represent a distinct species compared to North American strains, needing a possible reclassification of EEE (J. Virol. 84: 1014, 2010).

\section{Alphavirus Chikungunya (CHIK)}

Antigenically related with the next alphavirus; three genotypes of CHIK virus are differentiated: West African, and South/Central/East African, and Asian. In Svahili language, "chikungunya" means "something what causes people becoming bended".

Source of infection (natural host range): mammals (wild primates, bats), man. Animal disease: asymptomatic course.

Transmission mode (Fig. 8.3): culicine mosquitoes (Aedes aegypti, Ae. albopictus, Mansonia spp.). The natural cycles are: (a) sylvatic (gray vervet monkeys, baboons + canopy mosquitoes Ae. africanus, Ae. furcifer, Ae. taylori); (b) urban (synanthropic: man + Ae. aegypti, Ae. albopictus, Culex pipiens fatigans).

Human disease: chikungunya fever with a sudden onset and chills, strong headaches, severe arthralgia, myalgia, backache, nausea, vomiting, erythema on face and trunk, up to maculopapular rash, lymphadenitis, photophobia,

Fig. 8.3 The cycles of chikungunya virus (drawing by Ivo Rudolf)

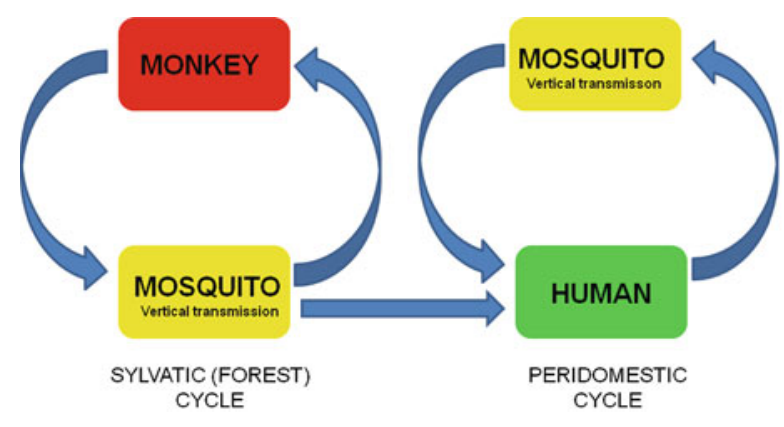


sometimes (e.g. with Asian strains) haemorrhages; fatality rate is very low, but arthralgia may develop into severe arthritis and persist for several months (even years). Epidemics of CHIK: 1952-1953, first recognized outbreak in eastern Africa; 1955, another big epidemic in Africa; 1964-1965, major outbreaks in India (at least half a million of cases) and Thailand; 2001-2003, a number of smaller outbreaks in Indonesia; 2004, an epidemic in Kenya; 2005-2006, a huge outbreak (about 280,000 cases including 213 fatalities) on islands of Indian Ocean which started on the Comoros and Réunion Island where 255,000 persons out of 775,000 inhabitants were infected (about 130 cases have been still reported in 2010); many additional cases were reported on the Seychelles archipelago $(9,000)$, Mayotte $(5,800)$, Mauritius $(6,000)$, Madagascar, and continued in India (Andhra Pradesh, Karnataka, Maharashtra - with a total of about 1.4 million of cases), later it also struck Sri Lanka, Malaysia, Thailand, Indonesia (even in 2009-2010 >12,000 people ill in province Lampung, SE. Sumatra), Madagascar and continental Africa and, moreover, tens of human cases (tourists etc.) were imported in Europe and North America from Asia; the vector in this epidemics was Ae. albopictus, and in India Ae. aegypti. In August and September 2007 broke out the first European epidemic (about 330 cases, 204 were laboratoryconfirmed) of chikungunya fever - in northern Italy (in the town Ravena and environs); the index case was a resident returning from India (he picked up the infection in Kerala, and arrived on 21 June); the local vector in Italy was Ae. albopictus, a mosquito species introduced into the area several years ago. Bio-containment: BSL-3.

Geographical distribution: tropical Africa (Uganda, Kenya, Tanzania, Mozambique, Zimbabwe, Malawi, Senegal, DR Congo), southern and SE. Asia (Photo 5.14).

\section{Alphavirus O'nyong nyong (ONN)}

Antigenically closely related to CHIKV. Igbo-Ora virus, isolated from febrile patients in Nigeria, is now considered a variant of ONN virus. In Svahili language, "o nyong nyong" means a "grinder or crusher/breaker of joints".

Source of infection (natural host range): mammals (wild primates, bats), man. Animal disease: asymptomatic course.

Transmission mode: Anophelinae mosquitoes (Anopheles funestus, An. gambiae). The natural transmission of the virus also includes sylvatic and urban cycle as in chikungunya.

Human disease: o'nyong nyong fever, clinically indistinguishable from CHIK. Epidemics of ONN: 1959-1961, 2 million people infected in eastern Africa (Uganda, Kenya, Tanzania, Zambia); 1996-1997, a return of ONN after 35 years in Uganda, with hundreds of ill persons (60-80\% of population infected); the epidemic later spread into neighbouring Tanzania; 2004, a smaller outbreak in Kenya. 
Bio-containment: BSL-2.

Geographical distribution: tropical Africa (Uganda, Kenya, Tanzania, Zambia, Mozambique, Malawi, Senegal, DR Congo).

\section{Alphavirus Semliki Forest}

Synonym or a subtype: Zingilamo virus.

Source of infection (natural host range): rodents, insectivores (Atelerix albiventris), wild birds.

Animal disease: asymptomatic course.

Transmission mode: mosquitoes Aedes spp., Culex pipiens.

Human disease: febrile illness with severe persistent headache, myalgia, arthralgia, occasional encephalitis; weakness in convalescence. Numerous human cases have been observed.

Bio-containment: BSL-3.

Geographical distribution: tropical Africa.

\section{Alphavirus Mayaro}

Recent molecular analyses have recognized two lineages: genotypes D and L.

Source of infection (natural host range): mammals (largely monkeys -Alouatta, Colobus, Pithecia, Saimiri, Callithrix, and rodents), birds, reptiles (Ameiva, Tropidurus).

Animal disease: asymptomatic course.

Transmission mode: Culicinae mosquitoes (Haemagogus etc.). Natural (sylvatic) cycle: between mosquitoes and monkeys.

Human disease: Mayaro fever - dengue-like illness with sudden high fever, headache, severe arthralgia (lasting for several weeks and affecting principally ankles, wrists, and toes, but occasionally also major joints), arthritis, oedema, myalgia and rash; retroorbital pain, vomiting; fatal cases have not been reported. On average, 20-50\% of Indians in the Amazon river basin have antibodies to the virus. In 1955, the first epidemic was observed in Brazil and Bolivia, and since that time other three big and a number of smaller outbreaks have been reported - the last one in Belém, northern Brazil, with 105 patients, and a recent outbreak (2010) in Venezuela affected 77 people.

Bio-containment: BSL-3.

Geographical distribution: South America (Trinidad, Surinam, French Guyana, Columbia, Panama, Brazil, Peru, Bolivia, Venezuela).

\section{Alphavirus Sindbis (Synonyms: Ockelbo, Pogosta, Karelian Fever Virus; Babanki: An African Subtype)}

This virus is related to the American equine encephalitis viruses, it belongs to the WEE antigenic group. 
Source of infection (natural host range): birds (e.g. Turdidae), less often mammals (e.g. rodents, marmoset Callithrix) or amphibians.

Animal disease: inapparent course (sometimes symptoms in pigeon, chicken and rodents).

Transmission mode: largely mosquitoes (Culex spp. - e.g. Cx. univittatus, $C x$. pipiens, Cx. torrentium, Culiseta spp.). Principal cycle in nature: between ornithophilic mosquitoes and birds (for instance, $27 \%$ of resident tetraonids Tetrao urogallus, T. tetrix, Bonasa bonasia, Lagopus lagopus in Scandinavia were found with antibodies in 2003).

Human disease: Sindbis fever (syn. Karelian/Ockelbo/Pogosta fever in Fennoscandia) with headache and arthralgia or arthritis, rash on thorax and extremities ("fever-arthritis-rash" triade); no fatal cases have been reported, but convalescence is long (the joints may be painful for months or even years; arthralgia, arthritis and rheumatic symptoms are observed in nearly $25 \%$ of patients for up to 3 years after the acute disease). Interestingly, the epidemics of Pogosta disease have repeated at least since 1974 (first noted epidemic) nearly regularly at the interval of 7 years, in a coincidence with the population cycles of tetraonid birds. In summary, extensive epidemics were recorded in 1974, 1981 (thousands of cases in Scandinavia and Karelia), 1988, and 1995. A total of 2,183 laboratory-confirmed cases were reported during 1981-1996. The last large epidemic in Finland took place in 2002 with almost 600 reported cases, while in 2009 only 105 laboratory-confirmed cases were reported. Seasonal peaks of the disease in Scandinavia are in the months August-September. Relatively frequent are Sindbis fever cases in African countries.

Bio-containment: BSL-2.

Geographical distribution: nearly worldwide (except for Americas).

\section{Alphavirus Ross River}

The RR virus was first isolated from mosquitoes in Australia in 1963.

Source of infection (natural host range): mammals (kangaroo Macropus agilis, brushtail possum Trichosurus vulpecula; rats; horse), birds (Grallina cyanoleuca, Microeca fascinans).

Animal disease: inapparent course.

Transmission mode: mosquitoes Aedes vigilax (coastal areas, TOT), Ae. tremulus (TOT), Culex annulirostris (inland), but also Verrallina funerea (competent vector), Ae. camptorhynchus, Ae. normanensis, Ae. polynesiensis, Ae. alterans, Ae bancroftianus, Ae. daliensis, Ae. flavifrons, Ae. funereus, Cx. quinquefasciatus, Cx. australicus, Cx. sitiens, Coquillettidia linealis, Anopheles amictus, An. annulipes, and other species.

Human disease: epidemic polyarthritis, with fever, headache, malaise, arthralgia (the joint pain involves fingers, toes, ankles, knees and elbows), myalgia, 
rash, apathy, lymphadenitis, affection of kidneys (haematuria, glomerulonephritis), splenomegalia; fatalities have not been reported; persistent or recurrent arthralgia and arthritis (polyarthritis) and lethargy can occur for up to 6-12 months. Ross River fever is the most frequent arbovirosis in Australia, with a mean incidence of about 4,800 patients annually (for instance 47,500 cases were reported in the period 1991-2000). Five big epidemics of RR occurred: in 1927/1928; during the 2nd WW; 1956; $1979 / 1980$ an explosive outbreak, with $>65,000$ persons affected on the Polynesian islands and archipelagos of Fiji, Samoa, New Caledonia, Cook and Tonga, and 2003/2004 (re-emergence on Fiji). Diagnostics and treatment of patients with RR fever costs Australia annually 2.7-5.6 million dollars. Risk factors for outbreaks include among others heavy rainfalls and higher maximum tides, causing increased mosquito population densities.

Bio-containment: BSL-2.

Geographical distribution: Australia, New Guinea, Polynesia (Solomon Islands, New Caledonia, Fiji, American Samoa, Tonga, Cook Islands).

\section{Alphavirus Barmah Forest}

The virus was first isolated from mosquitoes in the Barmah Forest in Victoria, Australia, 1974.

Source of infection (natural host range): mammals (e.g. kangaroo Macropus agilis), birds.

Animal disease: inapparent course.

Transmission mode: mosquitoes Aedes vigilax (competent vector), Culex annulirostris, Ae. funereus, Ae. camptorhynchus, Ae. normanensis, Ae bancroftianus, Ae. eidsvoldensis, Cx. globocoxitus, Verrallina funerea (competent vector), Anopheles amictus, An. annulipes, and other species.

Human disease: epidemic polyarthritis, with symptoms similar to those caused by Ross River virus - fever, headache, arthralgia, myalgia, rash (the rash more marked than in RR), apathy, lymphadenitis, affection of kidneys (haematuria, glomerulonephritis), splenomegalia; fatalities have not been reported; persistent arthralgia and polyarthritis occur for shorter time than in RR (a few months at maximum). First big epidemic of Barmah Forest arthritis was reported in 1993/1994 (but before, in 1992, also a number of cases occurred), and a total of 1,084 cases were reported between 1992 and 1999 (at the same time, the number of Ross River fever patients attained 5,863); a major epidemic occurred in 2005/2006, with 1,895 reported cases.

Bio-containment: BSL-2.

Geographical distribution: Australia. 


\subsubsection{Family Flaviviridae}

\subsubsection{Mosquito-Borne Flaviviruses}

\section{$\left.{ }^{* *}\right)$ Flavivirus Yellow Fever (YF)}

Virions of the genus Flavivirus [Lat. flavus, yellow] are spherical $(40-50 \mathrm{~nm})$, enveloped, they contain one molecule of ss(+)RNA sized 10-12 kbp, and surface proteins $\mathrm{E}$ and $\mathrm{M}$. This genus involves 75 viruses of 9 antigenic groups, most of them being transmissible by arthropods (mosquitoes and ticks). The strains of YF virus can be divided in Ethiopian and Neotropical (Theiler and Downs 1973), and according to sequencing analyses of genes for protein E in 3 genotypes: IA - West-African, IB - South-American, II - East-African. More detailed classifications differentiate even 7 genotypes.

Source of infection (natural host range): monkeys (species of the genera Colobus, Cercopithecus, Erythrocebus, Cercocebus, baboons, probably also galagoes in Africa, while Alouatta, Ateles, Saimiri, Pithecia, and Callithrix in South America), other mammals of tropical forests, opossum (Didelphis marsupialis); man (the main amplifying host of the virus in the urban cycle).

Animal disease: inapparent course (however, some species of New World monkeys die after experimental inoculation).

Transmission mode (Fig. 8.4): mosquitoes - Aedes aegypti (first demonstrated by the Walter Reed commission in 1902; TOT in Aedes spp. mosquitoes was demonstrated later) transmission to humans in the urban cycle, while "canopy" mosquito species Haemagogus spp. (in Brazil e.g. H. janthinomys) and Aedes spp. (in Africa e.g. Ae. africanus, Ae simpsoni) in the sylvatic (jungle) cycle between wild primates of the jungle ecosystem. In the sylvatic cycle, man acquires infection accidentally during occupational (e.g., hunters) or recreational (tourism) activities. In Africa, an intermediate (savannah) cycle involves transmission of YF from tree holebreeding Aedes spp. (e.g., Ae. opok, Ae. luteocephalus) to humans living or working in jungle border areas. For typical habitats of sylvatic YF, see Photos 5.1 and 5.2.

Human disease: yellow fever - high fever with chills, headache, backache, vomiting (in severe cases black vomit containing blood), haemorrhages, necrotic hepatitis (YF virus reveals a marked hepatotropism) with icterus, nephritis, albuminuria, sometimes also encephalitis; fatality rate is $5-40 \%$. YF was introduced in the Caribbean from western Africa during the slave trade (introduction of viraemic persons and infected Ae. aegypti mosquitoes on slave ships): the first extensive epidemic occurred here in 1647-1648 and involved Little Antilles (Barbados, St. Cristof, Guadeloupe), Yucatan and Cuba (in Havana one-third of citizens died); epidemics occurred frequently in Central America also in the eighteenth and nineteenth centuries. In 1741 and 1802-1803, big outbreaks of YF were reported in Portugal and Spain (80,000 victims - also an import on ships, with a consequent spread from seaports to inland); in 1793, 15\% citizens of Philadelphia died on YF; in 1853, 8,000 persons from 29,000 infected died in New Orleans; 1900, Havana: 


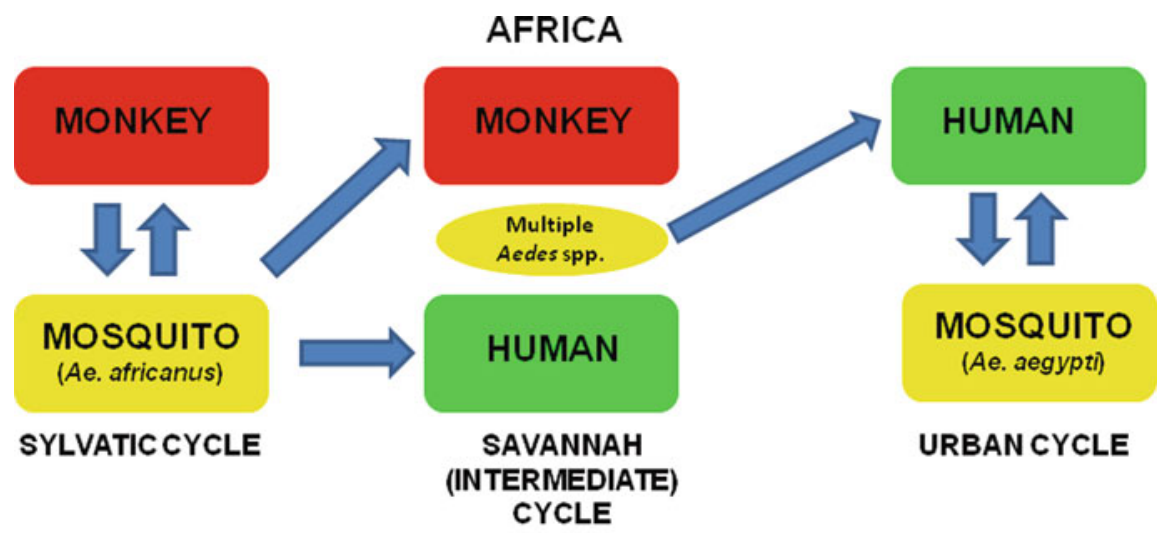

SOUTH AMERICA

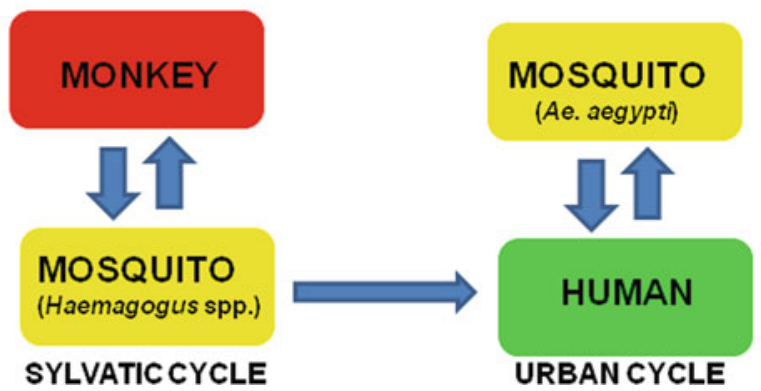

Fig. 8.4 Different cycles of Yellow fever virus (drawing by Ivo Rudolf)

Walter Reed Commission examined YF in Havana and evaluated the transmission mode of this disease; 1901, eradication of the Ae. aegypti vector of YF in Havana (Gorgas), later also in the Panama Canal area; since 1940 periodic epidemics have occurred in eastern Africa: 1940, Sudan 15,000 cases; 1951, Ethiopia 100,000 cases (30,000 people died); 1959, Sudan 1,800 cases; 1960-1962, Ethiopia 300,000 cases; 1965, Senegal 20,000 cases; 1969, Ethiopia 2,200 cases, and also a major epidemic in West Africa (Nigeria, Mali, Burkina Faso, Togo, Ghana); 1978 Gambia 8,400 cases (1,600 fatal); 1987, Nigeria 1,450 cases (565 fatal); 2000, Guinea 688 cases (225 fatal); 2005, Sudan 600 cases. 1998-2002, Brazil a total of 251 cases (96 fatal). According to the WHO data of 2000, mean annual incidence of YF in Africa is about 180,000 cases (of them about 27,000 fatal), elsewhere c. 20,000 cases $(3,000$ fatal).

Bio-containment: BSL-3.

Prevention: attenuated vaccine (attenuated clonal strain 17D prepared by Max Theiler) is recommended for travel in endemic tropical countries.

Geographical distribution: tropical Africa and America (in the urban cycle mainly seaport areas). 


\section{${ }^{(* *)}$ Flavivirus Dengue 1-4}

Four virus species (sometimes regarded as serotypes), each involves several genotypes. The name of dengue is a Spanish homonym derived from the Svahili "ki denga pepo", meaning "sudden convulsive attacks (cramplike seizure) caused by an evil spirit". First recognized (identified) in the 1950s during epidemics in the Philippines and Thailand.

Source of infection (natural host range): man (the main amplifying host of the virus), exceptionally monkey and other primates. Interestingly, RNAs representing the four DENV serotypes were detected in the livers and/or sera of wild mammals (rodents, marsupials, bats) caught in neotropical forests.

Animal disease: inapparent course, sometimes pathogenic for monkeys.

Transmission mode: mosquitoes Aedes aegypti, Ae. albopictus (TOT and sexual transmission in the mosquitoes), Ae. niveus in Malaysia, Ae. scutellaris in Polynesia, and other species; blood transfusion. Sylvatic cycle occurs in southeastern Asia - monkeys (Macaca and Presbytis spp.) + Ae. niveus; West Africa (Senegal, Nigeria - DEN-2) - monkeys Erythrocebus patas + Ae. furcifer, less often other species of Aedes (Ae.africanus, Ae. taylori etc.). For a habitat, see Photo 5.6.

Human disease: dengue, usually biphasic fever with an intense headache, myalgia, arthralgia, acute pain of eyes (retroorbital pressure), stiff neck, rash, nausea, insomnia; convalescence long (fatigue and depression for several weeks). Much more severe form of the disease is the dengue haemorrhagic fever (DHF), sometimes combined with syndrome of haemorrhagic shock (petechial bleeding into the skin and visceral organs) and fatality rate 5-20\%. DHF manifests most frequently when infection with DEN-2 virus follows about 20 years after primoinfection with DEN-1 virus (demonstrated in Cuba). Infection with one dengue serotype results in a long-term immunity against reinfection with the same type, there is, however, no cross-protection among other serotypes (i.e., a person could experience four independent infection). Dengue is an important tropical pandemic, comparable to malaria. Epidemics: 1897-1902 Australia; 1927/1928 Greece (650,000 persons from 704,000 citizens in Athens and Piraeus became ill); 1931 Tchaiwan; 1943-1944 Pacific islands and New Guinea - a frequent disease of American soldiers (a major health problem during war operations); 1954 the Philippines; 1980-1987 South and Central America recorded 40,000-390,000 cases annually (the Cuba alone reported >500,000 cases of DEN-1 in 1977, >400,000 cases of DEN-2 (DHF 10,000, 158 patients died on DHF shock) in 1981); and 5,208 cases of DEN-2 (DHF 205 cases, 12 patients succumbed) in 1997. Brazil reported 56,000 patients in 1994, 530,000 in 1998, and 290,000 in 2002 (all four dengue serotypes). In Argentina, dengue re-emerged in 1997, after the last epidemic in 1917. Dengue epidemics are less frequent in Africa, but the activity has increased also here: 1977 Seychelles; 1982 Kenya (DEN-2); 1985 Mozambique (DEN3); 1982 and 1993 Somalia (DEN-2); 1994 Saudi Arabia (DEN-2). Epidemic 
occurrence of dengue is also known from India, Singapore, Malaysia and elsewhere in tropical countries of Asia and Oceania. 1998 south Vietnam 120,000 cases (including DHF), 342 of them fatal. According to WHO, dengue occurs in $>100$ tropical and subtropical countries at present, about $40 \%$ of the world population is at risk, and approximately 50 million people are infected annually, 300-400 thousands have DHF and 22,000 die. Recent expansion of DEN-3 was reported in West Africa (Cape Verde Islands, 2009, more than 17,000 cases). According to WHO reports, about 160 per 100,000 tourists visiting endemic areas acquire dengue infection and export it worldwide annually.

Bio-containment: BSL-2.

Vaccine: not available at present.

Geographical distribution: the whole tropical and subtropical belt - largely southeastern Asian region (original area), Africa, and Central and South America, less often Mexico and southern Texas (2004), and since the 1990s also northeastern Australia.

\section{Flavivirus Japanese Encephalitis}

JE virus forms the antigenic group JE together with WN, SLE, MVE and some other viruses.

Source of infection (natural host range): birds (reservoir: colonial ardeids), pig (amplifier host), horse, bats.

Animal disease: abortions in swines, horse encephalitis (China).

Transmission mode (Fig. 8.5): mosquitoes of the genus Culex: Cx. tritaeniorhynchus (Japan, China - a common species in rice fields), Cx. pipiens group (Cx. pallens, $C x$. quinquefasciatus,), Cx. gelidus (a zoophilic species Malaysia), Cx. vishnui, $C x$. pseudovishnui, $C x$. japonicus. Many of them are species breeding in rice fields.

Fig. 8.5 The cycles of Japanese encephalitis virus (drawing by Ivo Rudolf)

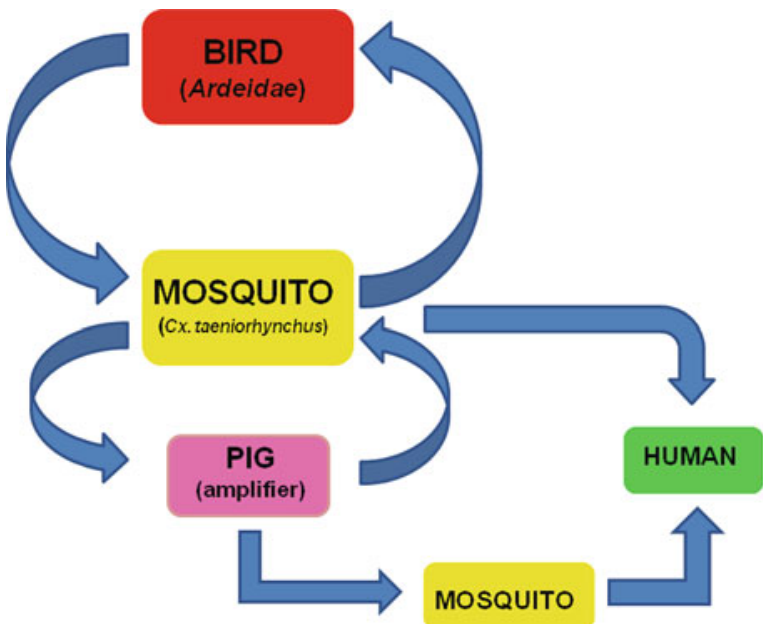


Human disease: Japanese encephalitis with fever, headaches, myalgia, decreased consciousness, convulsions, pareses, aggravated breathing; fatality rate $20-40 \%$; common are severe irreversible sequelae in $30-40 \%$ of patients (and even higher proportion among children). The first recorded extensive JE epidemic occurred in 1924, with 6,125 cases including 3,197 deaths. The present incidence in Asia is thousands of patients yearly: in the 1990s it was on average 45,000 (about 10,000 fatal), while in the last years 20-50 thousands annually, out of them 6,000-10,000 fatal (for instance, China reported a total of 38,000 cases in 1990 , about 15,000 in $1995,8,100$ in 1999 , and 5,104 cases with 214 deaths in 2005) (most of the deaths occurred in infants); India - Uttar Pradesh 4,544 cases (1,413 fatal) in 1990, and 5,737 cases (1,344 fatal) in 2005 .

Bio-containment: BSL-3.

Prevention: vaccine (inactivated Japanese strain Nakayama, or attenuated Chinese SA14-14-2).

Geographical distribution: east and southern Asia (Japan, Korea, China, India, Pakistan, Bangladesh, Nepal, Sri Lanka, Cambodia, Laos, Vietnam, Thailand, Indonesia, New Guinea). In northern Australia (Queensland) JE virus appeared in 2005 .

\section{Flavivirus West Nile (Subtypes: Kunjin, Rabensburg)}

Three genomic lineages of WNV are known at present (1-3), considered are two additional lineages (Indian and Caucasian), although the Caucasian (Russian) strain isolated from ixodid ticks seems to be closer to Koutango virus than to WNV. The Australian Kunjin is a member of the lineage $1 \mathrm{~b}$, most virulent are strains of the lineage $1 \mathrm{a}$.

Source of infection (natural host range): birds (waterbirds, corvids); occasionally probably rodents (Arvicanthis), equids, ruminants (camel), bats (Rousettus leschenaulti, Eptesicus fuscus), amphibians (Rana ridibunda) and reptiles (Natrix natrix, alligator). Migratory birds and corvids contribute to the spread of WNV to distant areas. Rabbits and squirrels have been shown experimentally to develop viraemia titres capable of infecting biting mosquitoes.

Animal disease: encephalomyelitis of equids with a high fatality rate, 25-30\% (France, Italy, Morocco, USA etc.). Virulent strains of WNV (e.g. NY-99) cause systemic disease in free-living birds (corvids - Photo 7.81 - and some other passerines, birds of prey etc.) and domestic birds (goose) with a mass dying, and also alligators in Florida were severely affected during the US epizootic.

Transmission mode (Fig. 8.6): largely by ornithophilic mosquitoes (Culex univittatus, $C x$. pipiens, $C x$. modestus, $C x$. quinquefasciatus, $C x$. salinarius, $C x$. restuans, etc.), exceptionally ixodid (Hyalomma) and argasid (Argas) ticks. WNV cycles: (1) sylvatic (exoanthropic, natural foci) involving wild, mainly wetland and water birds as amplifying hosts of the virus and ornithophilic 
Fig. 8.6 The cycles of West Nile virus (drawing by Ivo Rudolf)

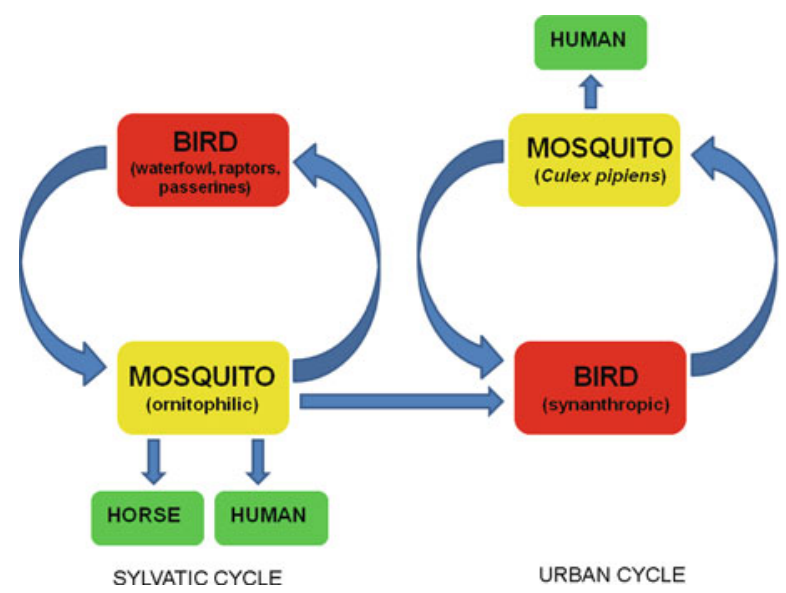

mosquitoes as vectors; (2) urban (synanthropic), with synanthropic and domestic birds as hosts and mosquito species feeding on both birds and mammals as vectors. Palaearctic natural foci of WNV infections are bound to wetland ecosystems, e.g. big river deltas (Volga, Danube, Rhône in Europe, or Djudj in west Africa). WNV transmission is also possible by blood transfusion, organ transplantation, or aerosol (laboratory infections). Contact and alimentary transmission between birds (raptors, corvids) has also been documented.

Human disease: West Nile fever (West Nile encephalitis, West Nile disease) with a sudden onset of fever, pharyngitis, headaches, myalgia, arthralgia, fatigue, nausea, sometimes conjunctivitis, lymphadenitis, quite often maculopapulose rash on the trunk and extremities with erythema in face, encephalitis (in about $10 \%$ of patients, i.e. roughly $1 \%$ of all infected persons); fatality rate is $5-10 \%$ (most frequently succumb persons older than 60 years). Convalescence used to be long in adults, but longterm sequellae are rare. Epidemics: 1950-1957 hundreds of cases in Israel, especially among immigrants; 1974 a big outbreak in South Africa (about 3,000 sick persons); 1994 Algeria, human cases; $1996>800$ patients (50 died) in Romania mainly in Bucharest and environs (in the consequent years 1997-2006 a total of only 90 cases with 6 fatalities); 1997 Tunisia, tens of human cases; 1997 south Moravia (Czechland) 5 cases; 1999 Volgograd, Astrakhan and Krasnodar regions 826 ill persons (40 died), and 2000-2006 here 316 additional patients; 1999-2000 Israel >430 cases (37 patients died); 1999 first introduction of WNV (Israeli strain) into USA - New York (62 ill persons, 7 of them died) while in subsequent years followed thousands of WNF cases in the USA (and several cases in Canada), and hundreds of patients succumbed (at the same time, thousands of horses and birds died as well); the virus spread rapidly across the continent - in 2002 it reached western coasts of North America being detected then in virtually all US states 
Table 8.1 Spread of West Nile virus in the USA and its establishment in the country, 1999-2009numbers of affected and reported individuals (CDC data)

\begin{tabular}{lrrrrrrrrrrr}
\hline & 1999 & 2000 & 2001 & 2002 & 2003 & 2004 & 2005 & 2006 & 2007 & 2008 & 2009 \\
\hline US & 4 & 12 & 27 & 44 & 46 & 47 & 48 & 46 & 43 & 40 & 35 \\
States & & & & & & & & & & & \\
Humans & 62 & 21 & 66 & 4,156 & 9,862 & 2,539 & 3,000 & 4,269 & 3,630 & 1,356 & 722 \\
Died & 7 & 2 & 9 & 284 & 264 & 100 & 119 & 177 & 124 & 44 & 25 \\
Horses & 25 & 63 & 738 & 9,157 & 4,146 & 1,341 & 1,072 & 1,121 & 507 & 224 & 298 \\
Birds & 295 & 4,323 & 7,333 & 14,122 & 11,350 & 7,074 & 5,204 & 4,106 & 2,182 & 3,026 & 759 \\
\hline
\end{tabular}

(Table 8.1). WNV has also occurred in Mexico, Central America including the Caribbean, and in 2006 as far southwards as in Argentina. 2003 France, 2 human cases. 2004 several patients in western Siberia. In 2008, several human neuroinvasive WN cases were reported from Italy (3), Hungary (12), and Romania (2). In 2009, 11 human cases of WNV encephalitis were recorded in Italy (Emilia-Romagna), and recently a WNF outbreak has occurred in northern Greece where 164 cases including 14 deaths have been reported as of September 6, 2010.

Bio-containment: BSL-3.

Prevention: There is a WNV vaccine for horses, but not yet a vaccine for humans. However, human vaccine against JE cross-protects against WNV.

Geographical distribution: nowadays worldwide - mainly Africa, the Mediterranean region, south Asia, Australia and Indonesia; less often southern and central Europe, and since beginning of the twenty-first century also the Americas. For habitats, see Photos 5.3-5.5, 5.42.

\section{Flavivirus St. Louis Encephalitis}

Source of infection (natural host range): wild synanthropic (peridomestic) birds such as house sparrow, pigeon etc., bats (Tadarida brasiliensis), fox.

Animal disease: mostly an inapparent course.

Transmission mode (Fig. 8.7): mosquitoes of the genus Culex: Cx. quinquefasciatus in the urban cycle, $C x$. tarsalis (TOT), Cx. nigripalpus, and $C x$. pipiens in the rural cycle; SLEV was also isolated from Psorophora ferox.

Human disease: St. Louis encephalitis with a fatality rate up to $20 \%$ (higher mortality occurs especially in persons older than 55 years), but more commonly only fever or meningitis. In USA, tens of SLE cases occur annually. Epidemics: 1932-1933 USA 1,350 cases (266 fatalities, and isolation of SLEV from a patient); 1962 Florida; 1975 USA 1,815 cases; 1990-1991 Florida and SE. Texas 226 cases (11 fatal). A total of 480 cases including 8 deaths were reported in Cordoba, Argentina in 2005.

Bio-containment: BSL-3.

Geographical distribution: North America, sporadically Central (Panama, Trinidad, Jamaica) and South (Brazil, Argentina) America. 
Fig. 8.7 The cycles of St. Louis encephalitis virus (drawing by Ivo Rudolf)

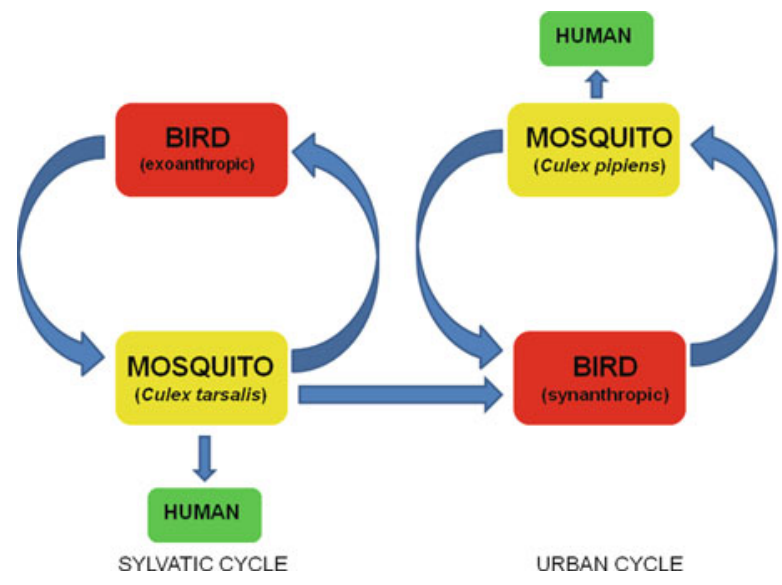

\section{Flavivirus Murray Valley Encephalitis}

Source of infection (natural host range): birds (especially waterbirds); (equids). Animal disease: usually inapparent course, sometimes encephalitis.

Transmission mode: mosquitoes (largely Culex annulirostris). In natural foci the virus circulates between wetland birds (herons, cormorants) and ornithophilic mosquitoes.

Human disease: Australian encephalitis (MVE), with fever, headaches, nausea and vomiting, fatality rate $20-60 \%$ and sequelae (paralysis of extremities, mental damage). Big epidemics were reported in 1917-1925 (>180 cases, $68 \%$ fatality rate), 1950-1951, 1956, 1971, and 1974. Outbreaks usually occur after heavy rains followed by extreme drought.

Bio-containment: BSL-3.

Geographical distribution: northern Australia, New Guinea.

\section{Flavivirus Bagaza}

Serologically it cross-reacts (in CFT) with distantly related WNV and JEV, being more closely related to Ntaya flavivirus. Interestingly, Bagaza virus might be genomically identical with Israel turkey meningoencephalitis virus.

Source of infection (natural host range): birds.

Animal disease: unknown in Bagaza virus (but Israel turkey meningoencephalitis is a serious veterinary disease).

Transmission mode: Culex spp. mosquitoes, e.g. Cx. neavei and Cx. poicilipes in Africa, and Cx. tritaeniorhynchus in India. In Senegal, the virus was also isolated repeatedly from Mimomyia mosquitoes (M. hispida, M. splendens, M. lacustris).

Human disease: fever and encephalitis; the disease has not yet been well characterised. Eight patients with encephalitis revealed neutralizing antibodies against Bagaza virus during an epidemic in the Kerala state (India) in 1996. 
Bio-containment: BSL-2.

Geographical distribution: Africa (Central African Republic, Cameroon, Senegal, Mauretania), India.

\section{Flavivirus Rocio}

Source of infection (natural host range): passerine birds.

Animal disease: inapparent course.

Transmission mode: by mosquitoes Psorophora spp.

Human disease: Rocio encephalitis with neurological and psychotic sequellae; fatality rate about 15 (5-30)\%. First epidemic recorded in Brazilian state Sao Paolo in 1975 (462 patients reported, 61 of them died; the total number of cases was however estimated at about 900).

Bio-containment: BSL-3.

Geographical distribution: South America (Brazil).

\section{Flavivirus Zika}

The closest relative is Spondweni virus (see below). Zika virus was first isolated from a febrile monkey caged on a tree platform in the Zika forest in Uganda, 1947.

Source of infection (natural host range): monkeys (Cercopithecus aethiops, Erythrocebus patas).

Animal disease: inapparent course.

Other mosquito-borne flaviviruses associated with human disease

\begin{tabular}{|c|c|c|c|c|c|c|}
\hline Virus & $\begin{array}{l}\text { Geographical } \\
\text { distribution }\end{array}$ & Vector & $\begin{array}{l}\text { Vertebrate } \\
\text { hosts }\end{array}$ & $\begin{array}{l}\text { Human } \\
\text { cases }\end{array}$ & Disease & BSL \\
\hline Banzi & South Africa & $\begin{array}{l}\text { Culex rubinotus, } \\
\text { Cx. neavei }\end{array}$ & $\begin{array}{l}\text { rodents, } \\
\text { possibly } \\
\text { also birds }\end{array}$ & 2 & Febrile illness & 2 \\
\hline Bussuquara & $\begin{array}{l}\text { Brazil, } \\
\text { Colombia, } \\
\text { Panama }\end{array}$ & Culex spp. & $\begin{array}{l}\text { monkeys; } \\
\text { birds? }\end{array}$ & 1 & Febrile illness & 2 \\
\hline Edge Hill & Australia & $\begin{array}{l}\text { Aedes vigilax, } \\
\text { Aedes spp. }\end{array}$ & wallabies & few & Febrile illness & 2 \\
\hline Ilheus & Brazil & $\begin{array}{l}\text { Aedes and } \\
\text { Haemagogus } \\
\text { spp. }\end{array}$ & primates & 8 & $\begin{array}{l}\text { Fever, CNS } \\
\text { signs }\end{array}$ & 2 \\
\hline Kokobera & Australia & Cx. annulirostris & birds? & 3 & Fever, arthritis & 2 \\
\hline Sepik & $\begin{array}{l}\text { Papua New } \\
\text { Guinea }\end{array}$ & $\begin{array}{l}\text { Mansonia } \\
\text { septempunctata }\end{array}$ & sheep & 1 & $\begin{array}{l}\text { Fever, } \\
\text { headache }\end{array}$ & 3 \\
\hline Spondweni & Tropical Africa & $\begin{array}{l}\text { Aedes } \\
\quad \text { circumluteolus }\end{array}$ & primates? & 7 & Febrile illness & 3 \\
\hline Usutu & Africa, Europe & Culex spp. & birds & 2 & Fever, rash & 2 \\
\hline Wesselsbron & $\begin{array}{l}\text { Africa, } \\
\text { Madagascar }\end{array}$ & Aedes spp. & $\begin{array}{l}\text { rodents, } \\
\quad \text { ruminants }\end{array}$ & 9 & $\begin{array}{l}\text { Biphasic fever, } \\
\text { CNS signs }\end{array}$ & 3 \\
\hline
\end{tabular}


Transmission mode: Aedes mosquitoes (Ae. furcifer, Ae. africanus, Ae. apicoargenteus, Ae. aegypti, Ae. luteocephalus, Ae. dalzieli).

Human disease: self-limited disease manifested by fever, maculopapular rash, arthralgia and conjunctivitis; other symptoms include malaise, back pain, diarrhoea and abdominal pain. In 2007, an extensive outbreak of Zika fever occurred on the Yap Island in Micronesia (first detection of the disease outside Africa and Asia), with at least 100 cases (49 were confirmed in laboratory, 59 were rated as probable). Prior to this epidemic, only about 14 human cases were reported in several African countries, India and Indonesia.

Bio-containment: BSL-2.

Geographical distribution: Africa (Uganda, Nigeria, Senegal, Tanzania, Central African Republic, Sierra Leone, Gabon, Egypt), Asia (India, Malaysia, Thailand, Vietnam, Indonesia), Oceania (the Philippines, Micronesia).

\subsubsection{Tick-Borne Flaviviruses}

\section{Flavivirus of Tick-Borne Encephalitis (CEE, LI, RSSE)}

There are four subtypes of TBEV: louping ill (LI); Central European encephalitis (CEE, e.g. topotype strains Hypr or Neudoerfl); Ural-Siberian, causing Russian spring-summer encephalitis (RSSE, e.g. strain Vasilchenko); and Far-Eastern (FEE, e.g. strain Sofyin). Dendrograms (Fig. 8.8) show antigenic and genomic similarity among viruses of the TBE complex: CEE virus is closer to LI virus than to RSSE virus, and thus LI should not be regarded as a separate virus, (because RSSE and CEE are considered as subtypes of one (TBE) virus). At the same time, Spanish sheep encephalitis virus is closely related to CEE virus.

Source of infection (natural host range): small forest mammals, especially rodents (Apodemus, Myodes, less often Microtus - CEE, RSSE, but probably not with LI virus), insectivores (hedgehog: CEE), goat (CEE) and sheep (CEE, LI), exceptionally cattle (CEE), certain forest birds (CEE, RSSE), red grouse Lagopus lagopus scoticus (LI) and mountain hare Lepus timidus (LI).

Animal disease: occasionally encephalitis in certain hosts (lamb, kid, dog, red grouse). LI virus causes disease in sheep and red grouse.

Transmission mode (Fig. 8.9): by ixodid ticks (reservoir - TOT), especially Ixodes ricinus (CEE, LI), I. persulcatus (RSSE); alimentary (consumption of raw, thermally untreated milk and dairy products from goats and sheep: 1951 Rožňava, Slovakia: about 500 patients), aerogenic (laboratory infections).

Circulation of TBE virus in a natural focus shows Fig. 8.9. About $0.5-3 \%$ of Ixodes ticks are usually infected in natural foci of TBE. For different natural foci of TBE (and LI), see Photos 5.7-5.13.

Human disease: tick-borne encephalitis usually with typical biphasic course: the 1st phase starts with fever and flu-like symptoms (severe headache, myalgia, arthralgia), sometimes conjunctivitis; after a short interval of usually 4-7 days of an apparent recovery, the 2nd phase starts with affection of the CNS (meningoencephalitis) accompanied with photophobia, dizziness, pareses of 

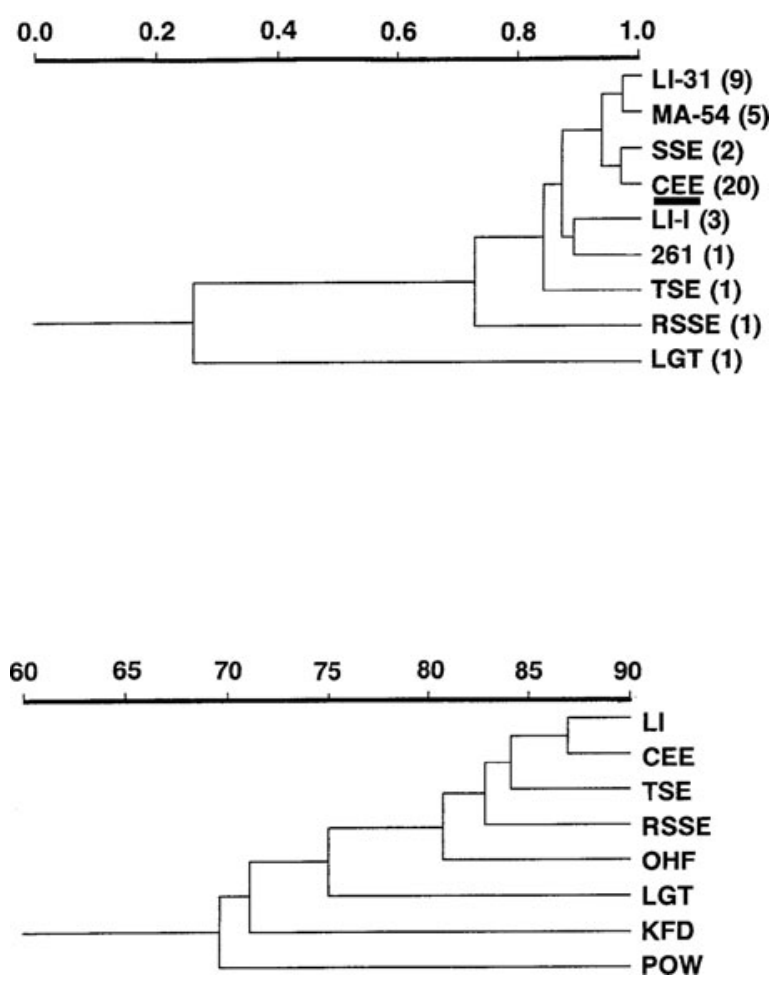

Fig. 8.8 Antigenic similarity among TBE complex viruses and strains based on IFA (the upper dendrogram: Acta Virol 39, 251-256, 1995). Louping ill strains are LI-31 (plus 8 related strains), MA-54 (plus 4 related strains; LI-I (plus two related strains), 261; SSE, Spanish sheep encephalitis; CEE, Hypr (plus 19 related strains); TSE; Turkish sheep encephalitis; LGT, Langat virus) The lower dendrogram: nucleotide sequence homology of the E gene among TBE complex viruses and strains (modified from Virus Res. 30: 129-144, 1993; and J. Gen. Virol. 75: 227-232, 1994) (drawing by Zdenek Hubalek)

cranial nerves and extremities, ataxia; fatality rate ranges from $1 \%$ (CEE, $\mathrm{LI})$ to $20-30 \%$ (RSSE, FE); convalescence is long-term, and neurological sequellae (residua) sometimes including pareses are quite common. Subtypes of TBE viruses were isolated in Eurasia first in 1930 (LI), 1937 (RSSE) and 1948 (CEE). In some European countries, TBE morbidity is quite frequent: for instance, on average 368 (140-744) cases of TBE was reported in Czechland (population about 10 million) annually in the two-decade period 1970-1999; In the year 2006 the TBE incidence in Czechland peaked at 1,026 patients. In 1999, an unusual epidemic of TBE (caused by a variant of FE subtype) occurred in the Novosibirsk area of Russia: out of 447 cases, 8 patients died with haemorrhagic syndrome. 1993-2000 were described first TBE human cases in Japan (caused by FE subtype).

Bio-containment: for CEE, RSSE and FE subtypes BSL-4 (when the personnel is vaccinated, BSL-3 is enough). For other TBE virus subtypes: BSL-3. 


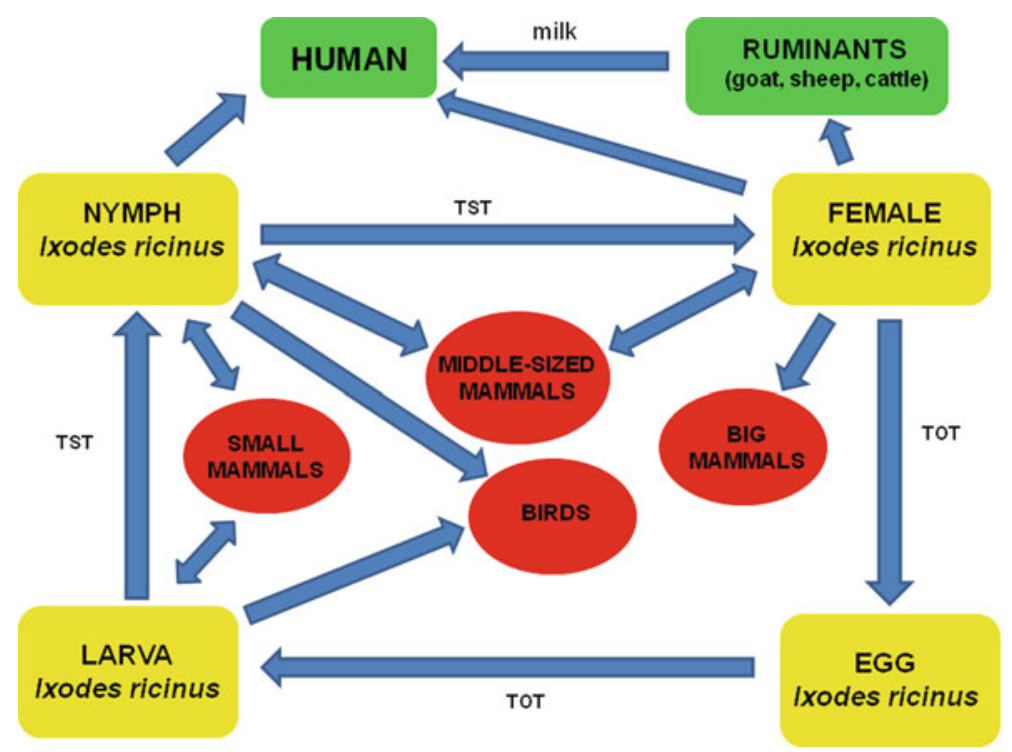

Fig. 8.9 Natural cycle of tick-borne encephalitis virus (drawing by Ivo Rudolf)

Diagnosis: serology (ELISA, HIT, CFT, VNT), detection of IgM in early phase or seroconversion in paired serum samples; less common is the isolation of the virus from the blood or CSF in cell cultures (e.g., PS pig embryo kidney cells) or in mice, detection of the virus' RNA by using RT-PCR.

Prevention: inactivated vaccine ("FSME-Immun" Immuno/Baxter; "Encepur" Behring); specific immunoglobulins (but effective only when applied immediately, i.e. within 1-2 days after infection, otherwise it could be even detrimental); repellents against ticks.

Geographical distribution: Eurasia (LI: UK and Norway, CEE: western and central Europe; RSSE: European and Asian Russia, Baltic countries, Finland; FEE: Far East Russia, northern China, Japan).

\section{Flavivirus Powassan}

This virus (with its subtype "Deer tick virus") is distantly related to TBE virus. The complete nucleotide sequence of the genome was determined (a total of 10,839 nucleotides).

Source of infection (natural host range): small and medium-sized mainly forest mammals, especially rodents (Marmota monax, Peromyscus leucopus, Tamiasciurus hudsonicus), skunk, fox.

Animal disease: inapparent course. Experimental inoculation of adult laboratory mice and Macaca mulatta monkeys caused their encephalitis and death.

Transmission mode: ixodid ticks (Ixodes cookei, I. marxi, Dermacentor andersoni). Virus is secreted in milk of experimentally infected goats. 
Human disease: Powassan encephalitis with fever, headache, prostration, meningitis and encephalitis; pleocytosis in CSF; spastic pareses, rarely paralyses and death (fatality rate about 10\%); neurological sequelae often persist. An infrequent disease in North America. For instance, 36 cases (without fatalities) were reported in the USA between 1958 (first human case) and 2005, but since the late 1990s, the incidence of human disease seems to be increasing.

Bio-containment: BSL-3.

Diagnosis: serology (ELISA, HIT, CFT, VNT).

Prevention: TBE vaccine could be cross-protective against Powassan virus infection.

Geographical distribution: North America (northern USA, Canada), Far East (Russia).

\section{Flavivirus Omsk Haemorrhagic Fever (OHF)}

The virus belongs to the so-called TBE complex. It was first isolated in 1947.

Source of infection (natural host range): rodents (muskrat Ondatra zibethica - imported to Siberia from Canada in 1928; Arvicola terrestris, Microtus gregalis), frogs and lizards.

Animal disease: occasional epizootics - mass dying of muskrats.

Transmission mode: ticks Dermacentor reticulatus, Ixodes apronophorus; alimentary (consumption of raw milk of goats and sheep, or drinking contaminated water); direct contact - e.g. in muskrat hunters.

Human disease: Omsk haemorrhagic fever - high fever, headaches, myalgia, nasal bleeding, pharyngitis, encephalitis (occasionally), haemorrhages; fatality rate 1-3\%; long convalescence. During 1988-1997, a total of 165 cases were reported from Siberia.

Bio-containment: BSL-4.

Diagnosis: serology (ELISA, VNT); virus isolation, RT-PCR.

Prevention: TBE vaccine might partially cross-protect.

Geographical distribution: steppe ecosystem in southern and western Siberia regions Omsk, Novosibirsk, Kurgan and Tyumen.

\section{Flavivirus Kyasanur Forest Disease (KFD)}

This virus also belongs to the so-called TBE complex. Very closely related to KFD virus (in fact, its subtype) is the virus Alkhurma (its overall genomic homology with KFDV is $89 \%$ ) and Nanjianyin virus (China).

Source of infection (natural host range): monkeys, rat Rattus blanfordi, striped forest squirrel Funambulus tristriatus, bats and insectivores (Suncus muri$n u s$ ) in KFD; and probably sheep and goat with Alkhurma virus in Saudi Arabia. 
Animal disease: occasional epizootics - mass dying of primates. For instance, high mortality due to KFD was observed in the black-faced langur (Semnopithecus entellus) and the red-faced bonnet monkey (Macaca radiata).

Transmission mode: ticks Haemaphysalis spinigera in KFD (reservoir - TOT demonstrated) and probably Ornithodoros savignyi in Alkhurma virus; alimentary (consumption of raw milk of goats and sheep, or drinking contaminated water).

Human disease: Kyasanur Forest disease - fever, headaches, pain in extremities, erythema on face, pharyngitis, encephalitis (in about $20 \%$ of cases), hepatitis, haemorrhages (nasal and gastrointestinal bleeding); fatality rate 8-15\%; long convalescence. Big outbreaks of KFD in Indian state Mysore in 1957, and Karnataka (213 cases with 14 fatalities) in 1986; the KFD foci activated in India in the 1990s; an average of 400-500 human cases have been reported annually over the last decades. In Saudi Arabia, 45 cases of Alkhurma haemorrhagic fever occurred in 2001-2003, the fatality rate was $25 \%$; additional 4 cases were reported in Makkah province in 2009. There is an occupational risk with Alkhurma virus (e.g., slaughtering of sheep).

Bio-containment: BSL-4.

Diagnosis: serology (ELISA, VNT); virus isolation, RT-PCR.

Prevention: TBE vaccine might partially cross-protect.

Geographical distribution: forests in India and West China - province Yunnan (KFD - Nanjianyin virus), and semidesert habitats in Saudi Arabia (Alkhurma).

\subsubsection{Bat-Borne Flaviviruses}

\section{Flavivirus Dakar Bat}

Source of infection (natural host range): bats Tadarida condylura, Scotophilus nigrita.

Animal disease: inapparent course.

Transmission mode: contact or aerogenic (?)

Human disease: febrile illness - two cases have been reported.

Bio-containment: BSL-2.

Geographical distribution: tropical Africa.

\section{Flavivirus Rio Bravo}

Source of infection (natural host range): the bats Tadarida brasiliensis, Eptesicus fuscus.

Animal disease: inapparent course.

Transmission mode: contact or aerogenic (?)

Human disease: febrile illness with respiratory involvement and lymphadenopathy - several cases have been reported.

Bio-containment: BSL-2.

Geographical distribution: Mexico and USA (Texas, New Mexico, California). 


\subsubsection{Family Bunyaviridae}

Virions are spherical (80-120 nm), enveloped, containing segmented molecule of a ss(-)RNA (the three segments are labeled L, M and S) sized 11-19 kbp (genus Orthobunyavirus - $12 \mathrm{kbp}$ ), with two surface glycoproteins G1 and G2, nucleoprotein $\mathrm{N}$ and transcriptase protein L. During a mixed simultaneous infection of cells in a vector or vertebrate host with different but related bunyviruses, reassortant (hybrid) virions can arise through incorporation of RNA segments from different viruses.

\section{Orthobunyaviruses of California Group: LaCrosse (LAC), California Encephalitis (CE), Snowshoe Hare (SSH), Ťahyňa (TAH), Inkoo (INK), Jamestown Canyon (JC)}

Another, a more distant member of this group is Guaroa virus. Ťahyňa virus (synonym: Lumbo) is the first mosquito-borne virus isolated in Europe (by V. Bárdoš and V. Danielová in 1958).

Source of infection (natural host range): leporids, rodents (Tamias, Spermophilus, Sciurus; Dicrostonyx), hedgehog, white-tailed deer (JC).

Animal disease: inapparent course.

Transmission mode (Fig. 8.10): mosquitoes of the genera Aedes and Culiseta (reservoir). LAC: Ae. triseriatus (TOT). CE: Ae. dorsalis (TOT, also sexual transmission in mosquitoes). TAH: Ae. vexans (TOT), Ae. caspius, Ae. cinereus, Ae. cantans and others; SSH, INK: Ae. communis, Ae. punctor, Ae. hexodontus.

Human disease: flu-like syndrome (e.g., Valtice fever: TAHV) lasting about a week, with fever, headache, myalgia, fatigue, lethargy, pharyngitis,

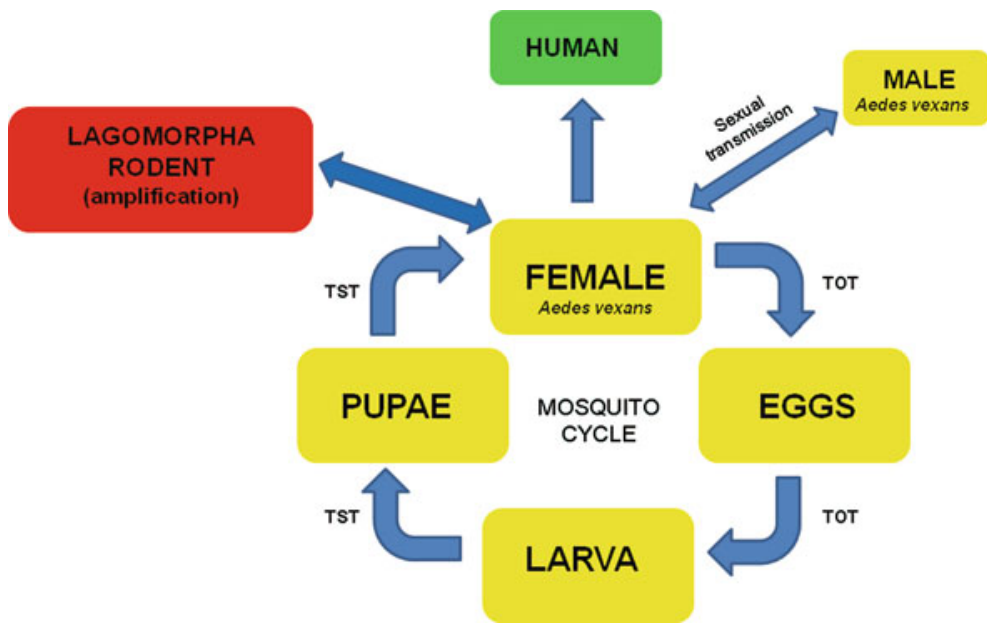

Fig. 8.10 Circulation of Ťahyňa virus in a natural focus of Valtice fever (drawing by Ivo Rudolf) 
conjunctivitis, nausea, gastrointestinal difficulties, sometimes also meningitis (TAHV) or encephalitis (California encephalitis: CEV, LACV), more common in children (however, $\overline{\mathrm{JC}}$ virus is more pathogenic for adults); fatalities are rare and occur in paediatric patients (LACV). The disease occurs usually in the late summer and early autumn when the vector mosquito density increases markedly. In the USA, the California group viruses are the most frequent cause of arboviral encephalitides (except for WN encephalitis since the year 2000). In the years 1963-1985, a total of 1,700 human cases of California encephalitis were reported from 23 states. During 2003-2007, a total of 355 patients ( $83 \%$ occurred in children $<16$ year old) were reported with probable and confirmed LACV infection in USA: $77 \%$ of them exhibited encephalitis or meningoencephalitis, $17 \%$ meningitis, and the rest were uncomplicated febrile cases; deaths occurred in $1.9 \%$ of confirmed cases, and in $8.6 \%$ of patients with encephalitis. In central and eastern Europe, many tens of cases of Valtice fever have been described since the 1970s, and a majority of people of the higher age groups living in, or close to, natural foci have antibodies to TAHV. However, many symptomatic cases of Valtice fever remain undiagnosed and characterized only as a (summer) status febrilis because there is no routine laboratory diagnosis of this disease in the region at present. Guaroa virus has been reported to cause 5 cases of febrile illness with headache, myalgia and prostration in Brazil.

Bio-containment: BSL-2.

Diagnosis: serology (ELISA IgM, HIT, VNT, IFA), exceptionally PCR or (technically more difficult) isolation attempts from the blood samples on Vero cells or on suckling mice.

Geographical distribution: Eurasia (TAH, INK) including China (TAH), Africa (TAH), North America (CE, LAC, SSH, JC), South America (Guaroa virus). Natural foci of TAHV infection in central Europe are characteristically situated in the floodplain-forest ecosystem with a mosaic of meadows and close arable fields (Photos 5.15 and 5.16).

\section{Orthobunyaviruses Batai (Synonyms: Čalovo, Olyka, Chittoor), Ilesha, Bwamba and Tensaw}

Source of infection (natural host range): livestock, domestic pig, primates.

Animal disease: usually inapparent course, some observed in goat and sheep.

Transmission mode: zoophilic mosquitoes of the genus Anopheles (An. maculipennis s.1., An. barbirostris, An. gambiae - Ilesha, Bwamba, An. gambiae - Bwamba, An. crucians - Tensaw virus), less Aedes and Culex spp. (Cx. gelidus).

Human disease: flu-like symptoms with fever, lethargy and lack of appetite. Incidence of this disease is low. The disease with Ilesha (7 cases) and Bwamba (9 cases) viruses included fever with headache, myalgia and rash of the observed Ilesha cases, one was fatal. One encephalitic case caused by Tensaw virus was reported. 
Bio-containment: BSL-2.

Diagnosis: serology (ELISA IgM, HIT, VNT, IFA).

Geographical distribution: Europe (Batai: Czechland, Slovakia, Austria, Hungary, Croatia, Serbia, Moldova, Ukraine, Russia, Finland, Sweden and Norway), Asia (Batai: Malaysia, India, China), central Africa (Ilesha, Bwamba), and southern USA (Tensaw: Florida, Alabama, Georgia).

\section{Orthobunyavirus Bunyamwera}

Antigenically related to Batai virus.

Source of infection (natural host range): monkeys, possibly rodents.

Animal disease: asymptomatic course.

Transmission mode: mosquitoes, mainly Aedes spp.

Human disease: febrile illness with rash - eight cases have been described.

Bio-containment: BSL-2.

Geographical distribution: tropical Africa.

\section{Orthobunyavirus Germiston}

Source of infection (natural host range): rodents.

Animal disease: asymptomatic course.

Transmission mode: Culex rubinotus.

Human disease: febrile illness with rash (3 cases), headache, backache, weakness and mental confusion.

Bio-containment: BSL-3.

Geographical distribution: tropical central, eastern and southern Africa.

\section{Orthobunyavirus Oropouche (Antigenic Group Simbu)}

Source of infection (natural host range): mammals (sloths, marsupials, monkeys Callithrix spp., man), birds.

Animal disease: inapparent course.

Transmission mode: biting midges (Culicoides paraensis - in urban cycle) and culicine mosquitoes (Aedes serratus, Culex quinquefasciatus). C. paraensis is the major biological vector of the virus to humans during urban epidemics of the disease. However, the vector(s) of the virus in its "silent" sylvatic cycle remain unknown.

Human disease: Oropouche fever - with chills, headache, myalgia, arthral-

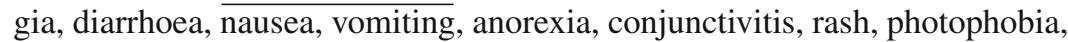
dizziness and meningitis or meningoencephalitis, leucopenia, infrequently haemorrhagic phenomena; no mortality nor sequellae have been reported. The virus was first isolated from the blood of febrile forest workers in Trinidad, 1954. A big outbreak (11,000 cases) occurred in Brazil in 1961; further Brazilian epidemics were reported in the years 1967/1968 and $1979 / 1980$ (200,000 cases), and a reemergence occurred in 2003/2004 and 
2006/2008 with several tens of cases. In 2010, 282 cases of Oropouche fever have been reported in Peru (as of August). A total of about 500,000 cases of Oropouche fever have been estimated to occur in 27 epidemics in Brazil, Peru and Panama since 1960; this is the second (after dengue) most frequent arbovirosis in South America.

Bio-containment: BSL-3.

Geographical distribution: South America - largely Amazonia (Brazil, Peru), Ecuador, Trinidad, and Central America (Panama).

\section{Orthobunyaviruses Guama and Catu (Antigenic Group Guama)}

Source of infection (natural host range): rodents (Oryzomys capito, Proechimys guyannensis and other spp.), monkeys, possibly also birds.

Animal disease: asymptomatic course.

Transmission mode: mosquitoes, mainly Aedes and Culex spp.

Human disease: febrile illness with headache, myalgia and arthralgia. Both viruses have caused 7-8 human cases each.

Bio-containment: BSL-2.

Geographical distribution: Brazil, Trinidad, French Guiana, Surinam, Panama.

\section{Bunyaviruses Apeu, Caraparu, Marituba, Murutucu, Nepuyo, Oriboca, Ossa and Restan (Antigenic Group C)}

Tropical mosquito-borne viruses.

Source of infection (natural host range): rodents (Caluromys philander, Proechimys guyannensis, P. spinosus, Oryzomys capito, O. laticeps, Didelphis marsupialis, Nectomys squamipes), monkeys (Cebus apella), and also fruit bats (Nepuyo virus: Artibeus jamaicensis, A. lituratus).

Animal disease: inapparent course.

Transmission mode: mosquitoes of the genera Aedes and Culex (Ae. arborealis, Cx. aikenii, Cx. portesi, Cx. vomerifer).

Human disease: up to 15 cases of a febrile illness have been reported for each of these viruses.

Bio-containment: BSL-2.

Geographical distribution: South America and Central America (tropical forests): Brazil, Trinidad, French Guiana, Surinam, Guatemala, Honduras, Panama, southern Mexico.

\section{Bunyavirus Keterah (Synonym: Issyk-Kul)}

This virus has not yet been assigned to a genus.

Source of infection (natural host range): bats; (birds?).

Animal disease: asymptomatic in green monkeys, but damage to visceral organs. 
Transmission mode: soft ticks (Argasidae), biting midges (Culicoides schultzei), and mosquitoes.

Human disease: a fever. An outbreak in Tadjikistan in 1982.

Bio-containment: BSL-3.

Geographical distribution: central Asia, Malaysia.

\section{Bunyavirus Bhanja (Synonym or Subtype: Palma)}

This virus has not yet been assigned to a genus.

Source of infection (natural host range): sheep, goat, cattle; hedgehog.

Animal disease: CNS affection in young ruminants (kid, lamb, calf).

Transmission mode: metastriate ticks (Haemaphysalis intermedia, H. punctata, Dermacentor marginatus, Hyalomma marginatum).

Human disease: fever, headache, conjunctivitis, sometimes meningoencephalitis. About 10 human cases have been described, one of them serious (quadriparesis).

Bio-containment: BSL-3.

Geographical distribution: Asia, Africa, southern Europe, eastern Slovakia. Examples of natural foci in Photos 5.18-5.21.

\section{Nairovirus Dugbe}

The virus is related to Ganjam virus (Nairobi sheep disease group).

Source of infection (natural host range): cattle (zebu), Cricetomys gambianus. Animal disease: not observed.

Transmission mode: Amblyomma variegatum and other metastriate ticks (Boophilus decoloratus and other Boophilus spp., Rhipicephalus muhsamae and other Rhipicephalus spp., Hyalomma marginatum, H. truncatum).

Human disease: fever, aseptic meningitis, thrombocytopenia. Two human cases have been reported.

Bio-containment: BSL-3.

Geographical distribution: tropical Africa - Nigeria, Senegal, Ivory Coast, Cameroon, Guinea, Central African Republic, Uganda.

\section{Nairovirus Crimean-Congo Haemorrhagic Fever}

It was recognized in 1969 that Congo virus, isolated in Africa, is identical to the agent of Crimean haemorrhagic fever, known in south Russia and southeast Europe, and Harry Hoogstraal thus proposed the combined name of the virus and disease $\mathrm{CCHF}$.

Source of infection (natural host range): leporids, hedgehog, other small mammals, cattle, horse, goat, sheep.

Animal disease: inapparent course. 


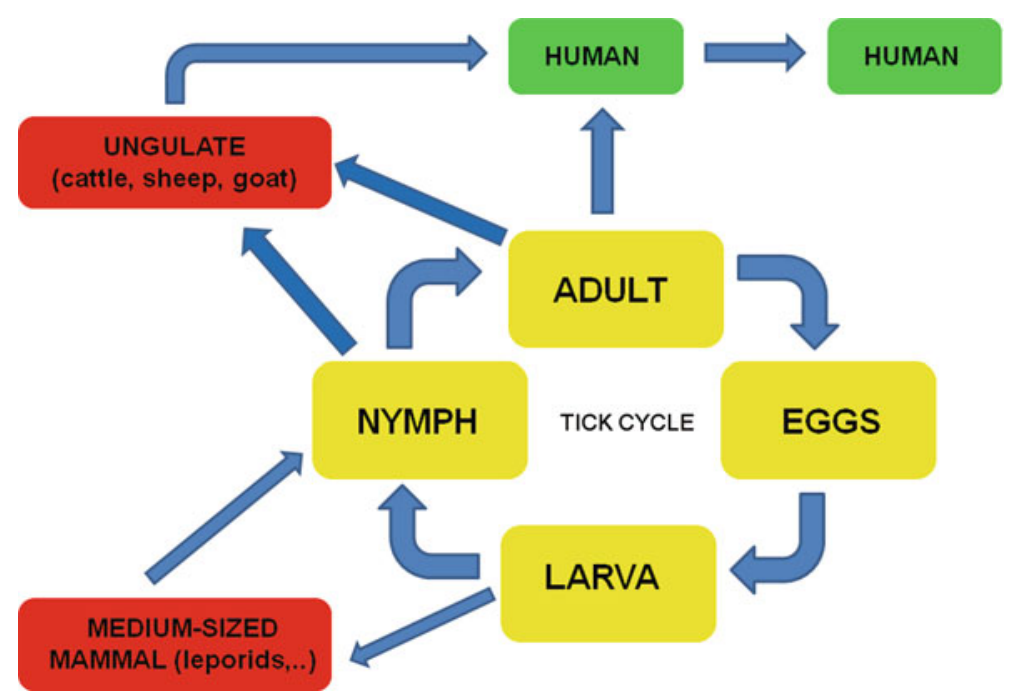

Fig. 8.11 Natural cycle of CCHF virus (drawing by Ivo Rudolf)

Transmission mode (Fig. 8.11): mostly bites by metastriate vector ticks - Hyalomma marginatum, H. anatolicum, Dermacentor marginatus, Rhipicephalus bursa and other spp. (reservoir - TOT; also venereal transmission in some species), frequently also by contact (nursing and care for patients, removal of feeding vector ticks, slaughtering of infected animals or those with attached ticks, sheep shearing). Occupational disease: cattle breeders, butchers, livestock industry, health professionals (nosocomial spread), specialized infectious laboratory workers (aerosol).

Human disease: Crimean-Congo haemorrhagic fever with severe headache, neckache, back pains, myalgia, dizziness, stiffness, petechial rash on the trunk, conjunctivitis, abdominal pain, vomiting, diarrhoea, photophobia, lymphadenopathy, hepatomegaly, hepatitis, psychotic signs (depression, sleepiness, lassitude), bleeding from mucous membranes (nose, gums, gastrointestinal tract) and kidneys, sometimes bleeding into brain, liver failure, pulmonary failure, haemorrhagic shock. Increased levels of transaminases, leucopenia, thrombocytopenia and coagulopathy. Long convalescence. Fatality rate 5-30\% (in nosocomial infections up to 50\%). Epidemics: e.g. in Bulgaria 487 notified cases in 1954/1955; Kosovo 119 cases (13\% fatal) during 1995-2001; 1997-2003 Bulgaria 138 cases (29 died); a total 1,568 cases were notified in Bulgaria from 1953 to 2008, with a mean fatality rate of 17\%. Since 1999 (but especially in 2006-2007), a reactivation of natural foci and re-emergence of CCHF occurred in southern Russia (regions Stavropol, Astrakhan, Rostov, Volgograd, Kalmykia, Dagestan) - a total of $>1,300$ patients were diagnosed with CCHF until 2007, the fatality rate has been $3-5 \%$. Albania reported 8 cases in 2001 and additional cases (an 
outbreak) occurred in Kosovo at the same time. A continuous epidemic process started in Turkey in 2002, and until 2009 a total of 4,430 cases were reported from 680 settlements mainly in the Tokat and Sivas provinces (but as many as 2,615 cases were notified solely in the last 2 years 2008 and 2009 ), with a mean overall fatality rate of $5 \%$; in addition, $16 \%$ of healthy population have antibodies to CCHF virus in Turkey at present (most often farmers and village residents). This exceptional epidemiological upsurge of CCHF in Turkey (largely in the Asian part of the country) has been associated ecologically with fragmentation and use of agricultural land and the formation, by this way, of optimal habitats for Hyalomma marginatum vector ticks. Some epidemics outside Europe: 2000-2004 Iran 248 cases; 2003 Mauretania 38 cases (11 fatal). In 2009, cases of CCHF were also reported from southern Russia, Georgia, Kazakhstan, Tajikistan, Iran, and Pakistan. Several US soldiers acquired CCHF during field operations in Afghanistan.

Bio-containment: BSL-4.

Diagnosis: RT-PCR, detection of antibodies or antigen (ELISA, IFA), isolation of the virus (extreme risk).

Treatment: in acute phase (if diagnosed very early) ribavirin, or hyperimmune serum (globulin).

Prevention: a vaccine of Bulgarian provenience (inactivated, suckling mouse brains; not commercial, a small scale production).

Geographical distribution: Africa, Arabian peninsula, Minor and central Asia, Iran, Pakistan, China (Xinjiang province), Albania, Kosovo, Bulgaria (Photo 5.17), Turkey, Greece, south Ukraine, south European Russia.

\section{Phlebovirus Rift Valley Fever (Synonym Zinga)}

Source of infection (natural host range): ruminants (sheep, goat, cattle, camel) - viraemia up to $10^{10} \mathrm{PFU} / \mathrm{ml}($ !), rodents.

Animal disease: sheep and goats, less often cattle suffer from necrotic hepatitis, abortions, teratogenic, often fatal (up to $90 \%$ of lambs die, but also adult sheep - c. 50\%). Big economic losses: e.g. in South Africa died about 100,000 sheep, and 500,000 ewes aborted in 1950. Susceptible (but the infection is inapparent) are equines, pig, dog, cat, guinea pig, rabbit.

Transmission mode (Fig. 8.12): mosquitoes Aedes spp. (TOT) (Ae. caballus, Ae. lineatopennis, Ae. vexans), Culex theileri, Cx. pipiens (Egypt), Cx. quinquefasciatus, Cx. poicilipes (Mauretania), Ae. caspius, less often (during epidemics) biting midges Culicoides, sandflies (strain Zinga of RVF virus) and other biting insects, and also ticks - e.g., Amblyomma variegatum on cattle in slaughterhouses; transmission also by direct or indirect contact with infected livestock, e.g. handling of sick animals and their tissue during slaughtering, disposal of carcasses, assisting with animal births (due to the high virus content in the blood and amniotic fluid of infected animals), aerogenic (e.g. laboratory infections), alimentary (consumption of raw milk, meat), iatrogenic (blood transfusion, injections). 


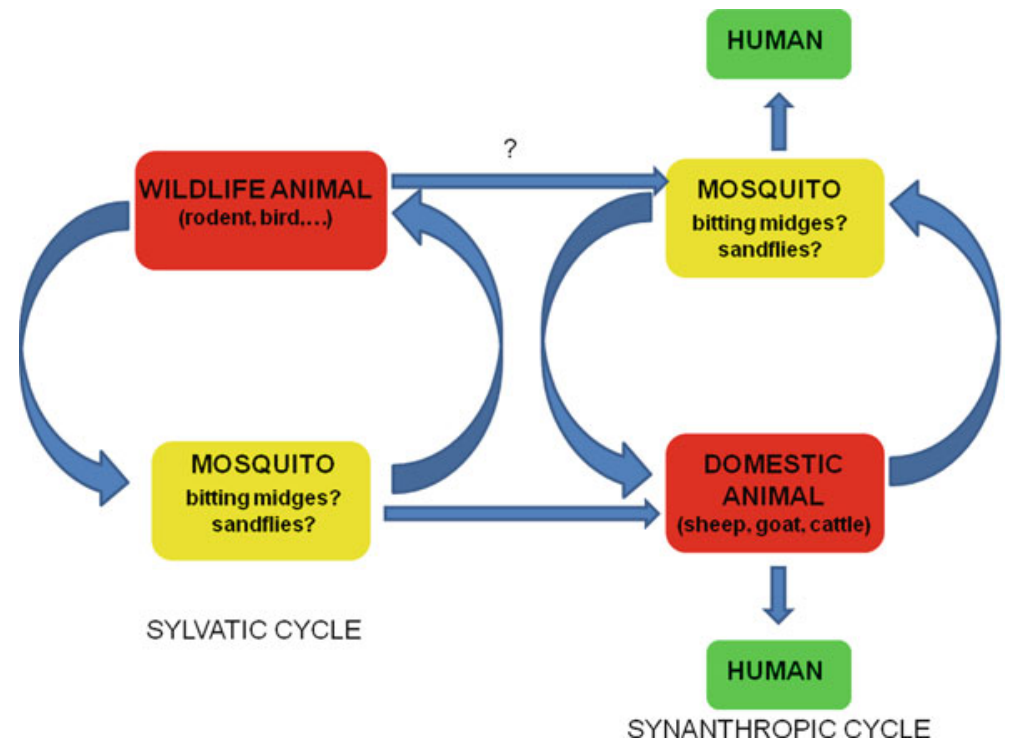

Fig. 8.12 The cycles of RVF virus (drawing by Ivo Rudolf)

Human disease: Rift Valley fever, usually biphasic course, severe headaches, arthralgia, photophobia, vision defects (up to blindness), retinitis, necrotic hepatitis, thromboses, sometimes haemorrhages (bleeding from nose) and encephalitis, occasionally lethal (fatality rate about 3\%). Occupational risk in herders, farmers, abbatoir workers, butchers, veterinarians. Epidemics of RVF have occurred in Kenya since beginning twentieth century, however the agent was identified only in 1930-1931; an outbreak in 1950-1951, with about 100,000 sheep and cattle, and 20,000 human cases; 1977-1978 Egypt 18,000 cases (600 fatal), but as much as about 200,000 persons were demonstrated serologically to be infected; 1980, South Africa about 20,000 cases; 1987-1988 Mauretania (220 patients died); 1993 and 1997-1998 Egypt, Somalia, Tanzania, Kenya and Mauretania - 90,000 cases (770 fatal); 2000-2001 for the first time in Arabian peninsula - Yemen 1,087 cases (121 died) and Saudi Arabia 884 diseased persons (124 died); 2003 Mauretania min. 25 cases ( 4 fatal). More recent outbreaks: Tanzania and Kenya 2007, Mayotte 2007-2008, Somalia 2010, an extensive outbreak affected livestock and humans in South Africa in 2010 (42 human laboratory-confirmed cases including 2 deaths).

Bio-containment: BSL-3.

Treatment: ribavirin helps when applied early.

Prevention and risk avoidance: vaccine (inactivated). Movement of cattle, goat and sheep herds is hazardous in epidemic situation. Cooking animal products before eating them is highly recommended. As a part of surveillance, reporting of abortion in cattle, sheep and goats and mortality in young animals is important. 
Geographical distribution: Africa (Kenya, South Africa, Sudan, Egypt, Zimbabwe, Mozambique Zambia, Senegal (Photos 5.24 and 5.25), Nigeria, Burkina Faso, Mauretania), Arabian peninsula.

\section{(**) Phlebovirus of Sandfly Fever: Sandfly Fever Naples (SFN), Sandfly Fever Sicilian (SFS), Toscana (TOS)}

Source of infection (natural host range): man.

Transmission mode: sandflies (Phlebotomus spp. - reservoir, TOT).

Human disease: sandfly fever, pappataci fever 2-4 days long, with lethargy, headaches and arthralgia, photophobia, retrobulbar pain, and heavy sweat; not lethal. Toscana virus causes also aseptic meningitis, while only sporadically encephalitis. Sandfly fevers escape attention and are most probably underdiagnosed.

Bio-containment: BSL-2.

Geographical distribution: the Mediterranean (Italy, Spain, southern France, Croatia - Dalmatia, Greece, Cyprus, Egypt; Photos 5.22 and 5.23), Portugal (TOS), Black Sea region (Crimea, Bulgaria, Turkey), central and south Asia. Common infections in holidaymakers or other visitors of the Mediterranean from central and northern Europe.

\section{${ }^{(* *)}$ Phleboviruses Chagres and Punta Toro}

Source of infection (natural host range): man, possibly also rodents.

Transmission mode: sandflies (Lutzomyia spp. - reservoir).

Human disease: fever with chills, headache, myalgia, retroorbital pain, vomiting - four cases have been reported with Chagres, and two other with Punta Toro virus.

Bio-containment: BSL-2.

Geographical distribution: Panama.

\section{Old World Hantaviruses: Hantaan, Dobrava, Saaremaa, Puumala, Seoul}

The name of the prototype virus is derived from the river Hantaan in Korea. Saaremaa virus is closely related to Dobrava (syn. Belgrade) virus. The number of all known hantaviruses approaches 45 at present, but a number of them (e.g. Tula virus, common in Eurasia) are practically nonpathogenic for man.

Source of infection (natural host range): murine and microtine rodents Apodemus agrarius (Hantaan, Saaremaa), A. flavicollis (Dobrava), A. ponticus (Dobrava), A. peninsulae (Amur), Myodes glareolus (Puumala), Microtus arvalis (Tula), M. fortis (Khabarovsk), Rattus rattus, R. norvegicus and Mus musculus (Seoul), Lemmus sibiricus (Topografov), etc. The rodents are not only the competent hosts of hantaviruses, but at the same time also the reservoir, due to their persistent latent infection and long-term excretion of hantaviruses by saliva, urine and faeces. 
Survey of human pathogenic Old World hantaviruses

\begin{tabular}{llll}
\hline Virus & Main rodent reservoir & Disease & Disease distribution \\
\hline Hantaan & Apodemus agrarius & HFRS & Asia \\
Saaremaa & Apodemus agrarius & HFRS & East Europe \\
Dobrava & Apodemus flavicolis & HFRS & The Balkans \\
Amur, Far East & Apodemus peninsulae & HFRS & Far eastern Russia \\
Seoul & Rattus norvegicus, & HFRS (mild) & Mainly Asia \\
& R. rattus & & \\
Puumala & Myodes glareolus & HFRS (mild) & Europe \\
\hline
\end{tabular}

Animal disease: inapparent course.

Transmission mode (Fig. 8.13): aerogenic (dried particles of rodents' infectious excreta), contact (rodent bite), and alimentary - from rodents. Hantaviruses are thus not arboviruses, but "rodent-borne" viruses.

Human disease: haemorrhagic fever with renal syndrome (HFRS: Hantaan virus - Korean haemorrhagic fever, Dobrava virus) with fever, headache, backaches, nausea, abdominal pain, vomiting, oedema and petechiae in mouth cavity and on face, later haemorrhages in gastrointestinal and urogenital tract, proteinuria, haematuria, renal insufficiency, kidney failure; fatality rate about 5\% (higher one in Asia); a milder form, nephropathia epidemica (NE, Puumala, Saaremaa), often with myopia but rare fatalities, occurs

Fig. 8.13 Natural cycle of hantaviruses (drawing by Ivo Rudolf)

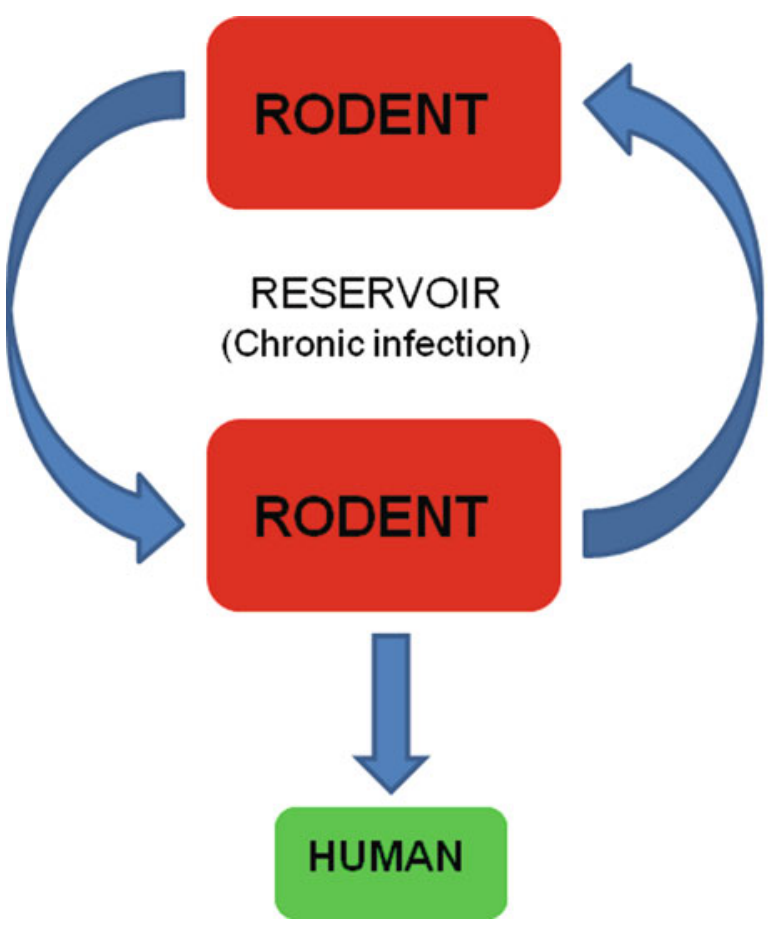


in Scandinavia, Belgium, the Netherlands, France, Germany, central and eastern Europe. HFRS was first reported in Korea in 1934 (Korean haemorrhagic fever); during the Korean War in 1951-1954, about 2,500 US soldiers acquired HFRS, and 120 of them died; Korea 1955-1957: 9,000 cases registered; on average, about 100,000 cases of HFRS are reported annually in China (some of them caused by Seoul virus); in the 1950s, several fatal cases of HFRS were also reported in east Slovakia and Serbia. Annual number of hantavirosis cases (mainly NE) in Europe including European Russia approaches 100,000 by expert estimations. In Sweden occur usually 200-600 NE cases annually, but in 2007 it was $>1000$ cases. An unusual epidemic of Puumala infection appeared in the urban park in Cologne (Germany) where 89 patients were reported in 2005. As many as 736 persons were infected with Puumala virus in Germany from January to August 2010.

Bio-containment: BSL-3.

Diagnosis: serology (ELISA, IFA), RT-PCR, immunohistochemistry (biopsy). Treatment: ribavirin helps when applied early.

Geographical distribution: Eurasia. (Photos 5.26 and 5.27)

\section{New World Hantaviruses: Sin Nombre, Andes, Choclo, Lechiguanas, etc.}

Source of infection (natural host range): neotomine and sigmodontine rodents - Peromyscus maniculatus and P. truei (Sin Nombre), P. leucopus (New York or Shelter Island virus), Oligoryzomys longicaudatus (Andes), O. fulvescens (Choclo), O. flavescens (Lechiguanas), Akodon azarae (Lechiguanas), Sigmodon hispidus (Black Creek Canal), Oryzomys palustris (Bayou) etc. Also these American species of rodents are not only the competent hosts of hantaviruses, but at the same time also the reservoir, due to their persistent infection combined with long-term excretion of the virus by saliva, urine and faeces.

Animal disease: inapparent course.

Transmission mode: aerogenic (dried particles of rodents' infectious excreta - urine, faeces, saliva), contact (rodent bite), and alimentary - food contaminated by infected rodents.

Epidemiological cycle is analogical to that of the Old World hantaviruses. The main risk factor for acquiring HPS or HCPS is being a "male farmer seeing or killing rats in the fields or dwellings".

Human disease: hantavirus pulmonary syndrome (HPS) - high fever, chills, headaches, myalgia, arthralgia, diarrhoea, nausea, sweat, thrombocytopenia, severe hypotension, cough, pneumonia (marked oedema of the lungs) intensive care including assisted ventilation is necessary, carditis (when also carditis is associated with the syndrome, the disease is called hantavirus cardio-pulmonary syndrome, HCPS); high fatality rate, 45-70\%. Sin Nombre: 186 cases in USA, 1993-1998. Andes virus has caused several epidemics with a high lethality; e.g., a total of 628 cases have been reported in Chile, with a mean fatality rate of $30-35 \%$. 
Survey of human pathogenic New World hantaviruses

\begin{tabular}{|c|c|c|c|}
\hline Virus & Main rodent reservoir & Disease & Disease distribution \\
\hline Laguna Negra & Calomys laucha, C. callosus & HCPS & $\begin{array}{l}\text { Paraguay, Bolivia, } \\
\text { Argentina }\end{array}$ \\
\hline Lechiguanas & Oligoryzomys flavescens & HCPS & Central Argentina \\
\hline Andes & Oligoryzomys longicaudatus & HPS & Southwest Argentina \\
\hline Bayou & Oryzomys palustris & HPS & Southeast USA \\
\hline New York-1 & Peromyscus leисориs & HPS & Eastern USA \\
\hline Sin Nombre & Peromyscus maniculatus & HPS & USA and West Canada \\
\hline Black Creek Canal & Sigmodon hispidus & HPS & Southern Florida \\
\hline Muleshoe & Sigmodon hispidus & HPS & Texas \\
\hline Araraquara & Bolomys lasiurus & HPS & Southeastern Brazil \\
\hline Castelo dos Sonhos & Unknown & HPS & Central Brazil \\
\hline Bermejo & Oligoryzomys chacoensis & HPS & Argentina, Bolivia \\
\hline Oran & Oligoryzomys longicaudatus & HPS & Argentina, Bolivia \\
\hline Anajatuba & Oligoryzomys fornesi & HCPS & Northern Brazil \\
\hline Rio Mearim & Holochilus sciureus & HCPS & Brazilian Amazon \\
\hline Rio Mamore & Oligoryzomys microtis & HPS & $\begin{array}{l}\text { Bolivia, Peru, French } \\
\text { Guiana }\end{array}$ \\
\hline Central Plata & Oligoryzomys flavescens & HPS & Southern Uruguay \\
\hline Choclo & Oligoryzomys fulvescens & HPS & Panama \\
\hline Calabazo & Zygodontomys brevicauda & HPS & South America \\
\hline Monongahela & Peromyscus maniculatus & HPS & North America \\
\hline
\end{tabular}

Bio-containment: BSL-3.

Diagnosis: serology (ELISA, IFA), RT-PCR, immunohistochemistry (biopsy). Treatment: ribavirin helps when applied early.

Geographical distribution: North, Central and South America - Sin Nombre virus in USA; Andes virus in Argentina, Uruguay and Chile, Choclo virus in Panama.

\subsubsection{Family Reoviridae}

Virions spherical (60-100 nm), they contain 10-12 segments of dsRNA with a total size of 19-21 kbp; reoviruses do not have lipoprotein envelope, and thus are insensitive to diethyl ether contrary to other arboviruses. They also do not produce haemagglutinin (HIT cannot be used in diagnostics). Due to the highly segmented genome, they are genomically (and phylogenetically) markedly variable.

\section{Coltivirus Colorado Tick Fever}

Virions $(60-80 \mathrm{~nm})$ contain 12 segments of dsRNA with a total size of $21 \mathrm{kbp}$.

Source of infection (natural host range): rodents (reservoir hosts: mainly Spermophilus lateralis, Tamias minimus, Erethizon dorsatum, Neotoma cinerea, Peromyscus maniculatus).

Animal disease: inapparent course (but teratogenic in mice). 
Transmission mode: ixodid ticks (Dermacentor andersoni, D. occidentalis, D. parumapertus, D. albipictus); iatrogenic - blood transfusion (the virus causes in humans persistent viraemia up to 120 days being localized in erythrocytes).

Human disease: Colorado tick fever (CTF) - usually biphasic, with headache, myalgia and arthralgia, conjunctivitis, photophobia, sometimes orchitis and affection of the CNS (mainly in children), temporary rash occurs less often (5-10\% of patients) than in RMSF, occasionally myopericarditis, pneumonia, hepatitis; leucopenia. Rare complications with this disease have included aseptic meningitis, encephalitis, and haemorrhagic fever. Laboratory findings include leucopenia, thrombocytopenia, and mildly elevated liver enzyme levels. Mortality is low, but the convalescence long (fatigue, lethargy).

Bio-containment: BSL-2.

Diagnosis: serology (IgM ELISA, IFA, VNT; not HIT); the virus is detectable since the 4th day of disease (and for a long time) in erythrocytes (by isolation in suckling mice, IF, PCR) - this is a specific pattern of CTF.

Geographical distribution: North America (natural foci in the Rocky Mts. USA, Canada, most often at altitudes of 1,200-3,000 $\mathrm{m}$ above sea level).

\section{Coltivirus Eyach}

Closely related to CTF virus.

Source of infection (natural host range): rodents and leporids.

Animal disease: inapparent course.

Transmission mode: ticks Ixodes ricinus and I. ventalloi.

Human disease: probably neuroinfections (not yet reliably demonstrated).

Bio-containment: BSL-2.

Diagnosis: serology (IgM ELISA, IFA, VNT; but not HIT); virus isolation.

Geographical distribution: Germany (1972), France; antibodies in several neurological patients in Czechland but the virus was not isolated. There is a hypothesis that this virus, a descendant of CTF agent, could have been imported from North America with U.S. Army dogs and their Dermacentor ticks to a military base situated in Germany after the 2nd WW, and evolved into Eyach virus under selective pressure of European ecosystems. Another hypothesis suggests that CTF virus could have been introduced in Europe with imported Sylvilagus floridanus cottontail rabbits, and evolved to Eyach virus.

\section{Orbiviruses Kemerovo and Tribeč}

Contrary to coltiviruses, orbiviruses of the Kemerovo group have only 10 segments of dsRNA with a total size of $19 \mathrm{kbp}$. Synonyms of Tribeč: Lipovník, Koliba, Cvilín, Brezová (subtype), Mircha, Kharagysh. 
Source of infection (natural host range): birds (European starling, chaffinch), rodents, goat.

Animal disease: inapparent course.

Transmission mode: ticks Ixodes ricinus and I. persulcatus.

Human disease: fever, sometimes meningitis.

Bio-containment: BSL-2.

Diagnosis: serology (CFT, VNT; but not HIT because these viruses do not form haemagglutinin).

Geographical distribution: Eurasia, exceptionally northern Africa.

\section{Orbivirus Orungo}

Source of infection (natural host range): probably primates including man.

Animal disease: not observed.

Transmission mode: Anopheles funestus, An. gambiae, and other mosquitoes.

Human disease: febrile illness with headache, conjunctivitis, myalgia, vomiting and rash - 60 cases have been reported.

Bio-containment: BSL-2.

Geographical distribution: central Africa and Senegal.

\section{Seadornavirus Banna}

This reovirus, a prototype species of the genus Seadornavirus, with a dsRNA genome consisting of 12 segments, is considered an emerging mosquito-borne virus in eastern and southeastern Asia. It was first isolated in China (Yunnan province) in 1987. Two groups (A, B) and subgroups (A1, A2) have been disclosed occurring in different areas.

Source of infection (natural host range): pig, cattle.

Animal disease: CNS affection in pigs.

Transmission mode: mosquitoes Culex tritaeniorhynchus, $C x$. pipiens, $C x$. pseudovishnui, Cx. modestus, Cx. annulus, Aedes vagus, Ae. albopictus, Ae. vexans, Ae. dorsalis, and Anopheles sinensis.

Human disease: encephalitis, with symptoms sometimes similar to Japanese encephalitis - febrile illness with myalgia and the CNS affection. The disease could be misdiagnosed as JE. Public health impact of this virus is probably underestimated.

Bio-containment: BSL-3.

Geographical distribution: China, Vietnam, Indonesia (from tropics to temperate ecosystems).

\section{Orthoreovirus Pulau (Synonym Melaka)}

The virus was first isolated from fruit bats in Tioman Island, Malaysia in 1999. Melaka virus, isolated in 2007 from a patient, and Pulau virus are genomically identical. 
Source of infection (natural host range): fruit bats.

Animal disease: not observed.

Transmission mode: aerogenically.

Human disease: high fever, sore throat and acute respiratory illness, with headache, myalgia, malaise, anorexia, and severe prostration - 3 cases were reported in Melaka, Malaysia, in 2007. Antibodies against the virus were found in about $13 \%$ inhabitants of the Tioman Island.

Bio-containment: BSL-2.

Geographical distribution: Malaysia.

\section{Rotavirus (Serotypes A and C)}

Virions of this genus of the family Reoviridae are spherical (80-100 nm), not enveloped, with 11 segments of dsRNA sized $19 \mathrm{kbp}$.

Source of infection (natural host range): cattle, pig (some genotypes of the serotype $\mathrm{C}$ of swine rotaviruses are obviously zoonotic; also zoonotic are some strains of serotype A which causes disease in small children under 5 years old), dog; man.

Animal disease: diarrhoea in calves and piglets.

Transmission mode: alimentary (this is, of course, not an arbovirus).

Human disease: gastroenteritis.

Bio-containment: BSL-2.

Geographical distribution: worldwide.

\subsubsection{Family Rhabdoviridae}

Virions are rod-like [Greek $\rho \alpha \beta \delta$ os (rhabdos) $=$ rod or rivet] with a characteristic bullet shape, about 100-500 $\times 45-100 \mathrm{~nm}$, enveloped, containing one molecule of ss(-)RNA sized 11-12 kbp.

\section{Vesiculovirus - Vesicular Stomatitis Complex (VSV) - VSI (Indiana), VSNJ (New Jersey), Alagoas, Piry, Chandipura}

Virions are $100-430 \times 45-100 \mathrm{~nm}$, the molecule of RNA has $11 \mathrm{kbp}$.

Source of infection (natural host range): equids, cattle, pig, wild mammals. Animal disease: vesicular stomatitis of cattle and horses.

Transmission mode: percutaneous, through conjunctiva, aerogenic, and also by haematophagous diptera (mosquitoes, sandflies [Lutzomyia trapidoi: TOT in VSI], biting midges, tabanids, blackflies). Non-viraemic transmission of VSV by co-feeding was experimentally demonstrated among blackflies.

Human disease: vesicular stomatitis - a short-term flu-like disease with myalgia, occasionally with vesicules in the oral and nasal cavities. Chandipura virus can cause encephalitis in children in India. 
Bio-containment: BSL-2.

Diagnosis: isolation on cell cultures, IF, serology (ELISA, IFA, VNT).

Geographical distribution: America, Africa (Senegal), India.

\section{Lyssavirus s.s.}

Genotype 1 of lyssavirus, rabies virus. Virions $100-435 \times 45-100 \mathrm{~nm}$, enveloped, containing one molecule of ss(-)RNA sized $12 \mathrm{kbp}$.

Source of infection (natural host range): canids and other carnivores (fox, dog, jackal, coyote, raccoon, skunk, badger Melogale moschata (China)); bats (in North America mainly Lasiurus cinereus and Myotis evotis while in South America Desmodus rotundus and other species of vampire bats). The source of human rabies were in Europe in the 1980s: wild mammals $78.8 \%$ (of them fox $74.1 \%$, badger and other mustelids $4.0 \%$, roe deer $3.9 \%$ ), cattle $9.4 \%$, $\operatorname{dog} 7.4 \%$ (the dog most often in Turkey, Greece, and Spain; in Czechland only $3.2 \%$ ), cat $4.3 \%$, man $0.1 \%$ (23 cases: the first patient mostly infected by a dog, 8 times through transplantation of cornea). In Africa, Asia and South America the dog prevails as the source of human infection ("dog rabies": the proportion is $75 \%$ and more), followed by domestic ruminants and man, while in North America, similarly as in Europe, wild mammals (76.5\%). According to the source of human infection we can distinguish:

(1) "urban (canine) rabies": main source are stray dogs; this form is dominating in Asia, Africa and South America, with mortality 11-83 human cases per 1,000 cases of rabies in animals;

(2) sylvatic (wildlife) rabies: main source are wild mammals; dominating in Europe and North America, with mortality $<1(0.3-0.5)$ human cases per 1,000 cases of rabies in animals.

Animal disease: lyssa, rabies - paralysis, aggressive behaviour. Rabies was also occasionally diagnosed in cattle, donkey, cat, roe deer, rabbit, and some rodents (squirrel, kangaroo rat, cotton rat, vole, hamster).

Transmission mode: percutaneous - biting of an infected mammal (virus in saliva), transplantation of cornea ( 8 cases), liver and kidneys ( 2 cases in Germany, infection of the donor originated in India).

Human disease: rabies (lyssa) - encephalomyelitis with excitation, aerophobia (patient cannot stand breath of air over face), hydrophobia, anxiety, hallucinations and bizzare aggressive behaviour, eventually followed by paralysis, coma and terminating with death at full consciousness (without treatment, the fatality rate is $100 \%$ ). Acute neurologic disease is divided into furious and paralytic (dumb) forms. Incubation period is long, 10 days to 6 months. The disease was known already in the Old Babylon, Egypt, Israel, India, China, ancient Greece and Rome. According to WHO, about 50,000-60,000 human deaths from rabies are estimated to occur annually, most often in Asia: e.g., 
about 20,000 cases in India (the Buddhist and Hindu ethics forbid culling the stray dogs; also in Thailand the killing of stray dogs is prohibited).

Bio-containment: BSL-2/3.

Diagnosis: anamnesis (animal bite); observation of the captured biting animal for the period of 5-10 days by a veterinarian, or post-mortem examination of the animal (histological slides of the brain and salivary glands - detection of antigen by the technique IF, Negri bodies or intracerebral inoculation of mouse); in the human patient, IF microscopy of corneal, mucosal and bioptic samples; also isolation on mice.

Treatment: post-exposure prophylaxis (PEP) is an immunization with several doses of vaccine combined with antiserum application (antirabic equine immunoglobuline).

Prevention: vaccination of exposed persons - several effective inactivated rabies vaccines are currently available worldwide (the virus is cultivated in human diploid cells); obligatory immunization of pet dogs, reduction of foxes or their vaccination (baited oral vaccine with strain SAD, suggested by George M. Baer); the latter method has $80 \%$ effectivity in the fox populations. Oral vaccination of foxes started in Europe in Switzerland (1978), and it has then been used in France, Germany, Czechland and other countries.

Geographical distribution: Lyssavirus s.s. occurs worldwide with the exception of Great Britain (the UK has applied a very strict policy since 1886: a 6-month quarantine for import or visit of dogs and cats), Ireland, Iceland, Japan, Australia, New Zealand and some other islands. The highest prevalence of the virus is in South and East Asia, and Central Africa.

\section{Bat Lyssaviruses}

Virions are similar to those of Lyssavirus s.s.

Genotype 2: Lagos bat virus (isolated from African bats Eidolon helvum, Micropterus pusillus, Epomophorus wahlbergi, Epomops dobsonii, and from the carnivore Atilax paludinosus) - probably not pathogenic to humans.

Genotype 3: Mokola virus (from African rodent Lophuromys sikapusi, shrews Crocidura spp.; also domestic dogs and cats) - few fatal human cases have been reported.

Genotype 4: Duvenhage virus (from bats Miniopterus schreibersii, Nycteris gambiensis, N. thebaica) - exceptional fatal cases in humans (South Africa 2006, Kenya 2007 - a Dutch tourist).

Genotype 5: European bat lyssavirus type 1 (EBLV-1: bats, mainly Eptesicus serotinus, further E. isabellinus, Tadarida teniotis, Myotis myotis, M. nattereri, Miniopterus schreibersii, Rhinolophus ferrumequinum); bat to cat transmission of EBLV-1 was documented in France (2003); two human fatal cases have been reported (Ukraine 1977, Russia 1985).

Genotype 6: European bat lyssavirus type 2 (EBLV-2: bats Myotis dasycneme, M. daubentoni) - two human fatal cases were reported (Finland 1985, 
Scotland 2002). Relatively closely inter-related are the genotypes 4 and 5, EBL1 is closer to Duvenhage than is EBL2.

Genotype 7: Australian bat lyssavirus (ABLV: fruit bats Pteropus alecto, P. scapulatus, P. poliocephalus, P. conspicillatus, Saccolaimus flaviventris) - two fatal human cases were reported in Queensland, 1996 and 1998.

Genotype 8: "Marmoset" lyssavirus (from the marmoset Callithrix jacchus kept as a pet animal in Brazil) - a total of eight fatal cases in humans in the years 1991-1998.

Some additional bat (Microchiroptera) lyssaviruses were isolated in Asia (Aravan, Khujand, Irkut, and West Caucasian) but their pathogenicity for man is unknown, no human cases have been reported.

Source of infection (natural host range): bats - EBL1 largely Eptesicus serotinus (in the Netherlands in 21\% from 1,219 bats of this species in 2005, also in central Europe), further Vespertilio, Nyctalus, less Miniopterus schreibersii, Rhinolophus ferrumequinum; EBL2 Myotis spp. (M. daubentoni, M. dasycneme); ABL fruit bat Aethalops alecto. Numbers of bats with demonstrated EBL1 or EBL2 in Europe, 1954-1992: the Netherlands 194, Denmark 164, Germany 76, Spain 7, former Yugoslavia 7, Poland 3, Sweden 3, France 2, Switzerland, Czechland, Russia and Turkey one each.

Animal disease: in bats - sometimes paralysis, aggressive behaviour.

Transmission mode: percutaneous - bites of an infected bat (virus in saliva); aerogenic (by aerosol in caves populated by rabies-infected bats).

Human disease: rabies (lyssa) - encephalomyelitis with excitation etc. Some of the bat lyssaviruses can kill humans (see above).

Bio-containment: BSL-2/3.

Diagnosis: anamnesis (animal bite); examination of the animal (histological slides of the brain and salivary glands - detection of antigen by IF or intracerebral inoculation of mouse); in the human patient, IF microscopy of corneal, mucosal and bioptic samples; also isolation on mice.

Treatment: post-exposure prophylaxis is an immunization with several doses of vaccine combined with antiserum application (antirabic equine immunoglobuline).

Geographical distribution: mainly in Europe and Africa, e.g. EBL1 in Poland, Czechland, Ukraine, Russia, Finland, France, Germany, Denmark, the Netherlands, former Yugoslavia, Spain; EBL2 in Great Britain, Finland, the Netherlands, Germany, Switzerland. However, some human pathogenic bat lyssaviruses occur also on other continents (see above).

\subsubsection{Family Arenaviridae}

Virions spherical to pleomorphic (85-130 nm), enveloped, contain two segments of $\mathrm{ss}( \pm)$ RNA sized $11 \mathrm{kbp}$. Interestingly, they also contain ribosomes (20-25 nm) that cause their "sandy" appearance under electron microscopy. Zoonotic arenaviruses 
belong to two complexes: (1) Old World (LCM-Lassa) complex with Murinae rodents as reservoir (with the viruses LCM, Lassa, Mopeia, Mobala, Dandenong, Morogoro, Ippy, Kodoko, and Lujo), and New World (Tacaribe) complex, with Sigmodontinae rodents as reservoir (with the viruses Tamiami, Whitewater Arroyo, Pichinde, Amapari, Flexal, Guanarito, Junin, Latino, Oliveros, Machupo, Parana, Pirital, Sabiá, Bear Canyon, Catarina, Allpahuayo, Chapare, Cupixi, and Tacaribe).

\section{Arenavirus Lymphocytic Choriomeningitis (LCM virus)}

Source of infection (natural host range): some species of wild and pet rodents (mainly Mus musculus and M. domesticus, but also M. spretus, Apodemus agrarius, A. flavicollis, A. sylvaticus, A. mystacinus, Micromys minutus, Microtus levis, Chionomys roberti, Myodes glareolus, Arvicola schermani, Mesocricetus auratus, Cavia porcellus) are reservoir of the infection (congenital infection with the long-life carriership and occasional excretion of LCM virus with saliva, faeces and urine, also horizontal and vertical transmission among rodents).

Animal disease: LCM (only in adult mice - young rodents, when infected, form subsequently an immunotolerant state with persistent infection).

Transmission mode: contact, percutaneous (biting), aerogenic, alimentary (contaminated food), congenitally (from infected mother to her foetus); organ transplantations.

Human disease: lymphocytic choriomeningitis - flu-like aseptic meningitis or only fever (usually biphasic) with headache, rhinitis, bronchitis, photophobia; miscarriage, embryo malformations (hydrocephalus, chorioretinitis); fatality rate about $1 \%$, convalescence long. Immunocompromised patients are highly susceptible to the infection. Occupational disease in pet rodent breeders. Dandenong is a new arenavirus related to LCM that was recently isolated from patients after transplantation from deceased donor, who had traveled in eastern Europe.

Bio-containment: BSL-2/3.

Diagnosis: serology (indirect IFA, ELISA, CFT, VNT).

Geographical distribution: probably worldwide.

\section{Arenavirus Lassa}

Source of infection (natural host range): rat Mastomys natalensis (reservoir: viraemia, virus excretion in urine; Photo 7.56), M. erythroleucus (in Sierra Leone), less often other rodents; man.

Animal disease: inapparent course (carriership - reservoir).

Transmission mode (Fig. 8.14): contact, alimentary (capture and consumption of Mastomys), aerogenic (aerosolized Mastomys urine); high contagiosity potential transmission to medical personnel who can acquire the disease in the hospital or in village settings from the index patients (blood, excreta).

Human disease: Lassa haemorrhagic fever with shivers (chills), pharyngitis, lymphadenitis, headaches, tinitus, myalgia, arthralgia, abdominal pain, diarrhoea, vomiting, rash, haemorrhages (bleeding into gastrointestinal tract 
Fig. 8.14 Natural cycle of Lassa virus (drawing by Ivo Rudolf)

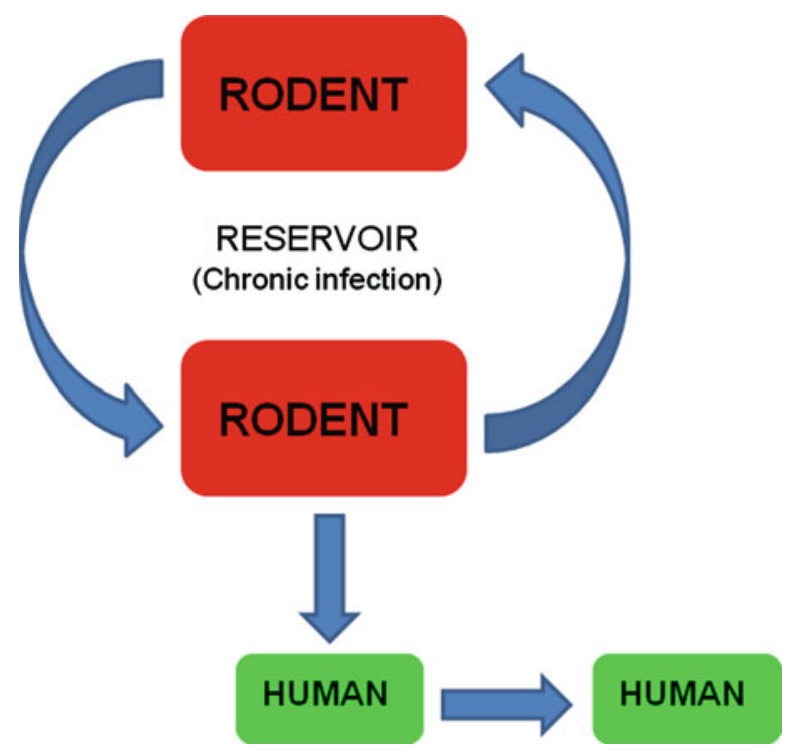

etc.), facial and neck oedema, tinnitus to deafness (common complication), hypovolaemia, abortion; fatality rate $10-50 \%$, in severe cases up to $70 \%$. In 1969-1975, a number of epidemics were recorded in tropical Africa (Nigeria, Liberia, Sierra Leone); hundreds of cases have been observed in northern Nigeria each year. 1996-1997 Sierra Leone: 823 cases, 53 died. In Sierra Leone, up to $40 \%$ of adult persons were found to be seropositive to Lassa virus in some areas (while the national mean seropositivity rate was 9\%). It is estimated that $100,000-300,000$ human infections per year occur in West Africa (with approximately 5,000 deaths). It is interesting that only 6-20\% of persons infected with Lassa virus become clinically ill (according to serological survey in the latter two African countries). Imported cases of Lassa fever are occasionally reported in tourists and hunters returning from endemic regions.

Bio-containment: BSL-4.

Diagnosis: ELISA (IgM and IgG), IFA, RT-PCR, immunohistochemistry (postmortem).

Treatment: ribavirin (helps only when applied in the first 3 or so days of disease: fatality rate decreases to 5-10\%); immune serum; supportive care (e.g. fluid and electrolyte balance, oxygenation, blood pressure monitoring).

Prevention: avoiding contact with Mastomys spp. rodents.

Geographical distribution: western and central Africa (Nigeria, Liberia, Sierra Leone, DR Congo, Mali, Guinea, Senegal and the Central African Republic).

\section{Arenavirus Lujo}

Source of infection (natural host range): rodents.

Animal disease: unknown. 
Transmission mode: contact or aerosol (highly contagious).

Human disease: haemorrhagic fever with severe headache, myalgia, vomiting, chest pain, sorethroat (pharyngitis), thrombocytopenia, granulocytosis; acute respiratory distress syndrome, and renal dysfunction. Outbreak of disease involving 5 patients, 4 of whom died, occurred in South Africa, 2008 (the first patient had been transferred from Zambia to South Africa for medical management, 3 cases involved secondary spill over of infection from the first patient, and one was a tertiary infection).

Bio-containment: BSL-4.

Diagnosis: ELISA (IgM and IgG), RT-PCR, immunohistochemistry (post mortem).

Treatment: ribavirin or immune plasma in the acute phase.

Geographical distribution: Zambia (South Africa).

\section{Arenavirus Junin}

Source of infection (natural host range): drylands vesper mouse Calomys musculinus (reservoir) - chronic infection with a persistent viraemia and excretion of the virus by urine, saliva and faeces.

Animal disease: inapparent course (but lethal in guinea pig).

Transmission mode: alimentary, contact (percutaneously - bites), aerogenic (in farmers); high risk of laboratory infections (aerosol).

Human disease: Argentine haemorrhagic fever - high fever, headaches, pain in abdomen and extremities, conjunctivitis, erythema on head and chest, petechial bleeding in the mouth, gastrointestinal and urogenital tracts, petechiae also in the skin, neurological defects (palsy, epilepsy), hypovolemic shock; fatality rate up to $30 \%$, very long convalescence. Epidemics and cases have occurred in Argentina since 1958 (discovery date); up to now a total of at least 25,000 clinical cases.

Bio-containment: BSL-4.

Diagnosis: ELISA (IgM and $\operatorname{IgG})$, RT-PCR, immunohistochemistry (post mortem).

Treatment: should start early with immune serum (plasma) and ribavirin.

Prevention: vaccine.

Geographical distribution: South America (Argentina).

\section{Arenavirus Machupo}

Source of infection (natural host range): rodents Calomys spp. (mainly C. callosus, reservoir) - chronic infection with persistent viraemia, excretion of the virus via urine, saliva and faeces.

Animal disease: inapparent course (but lethal for guinea pig).

Transmission mode: alimentary, contact (percutaneous - bites), aerogenic (in farmers); exceptionally high risk of laboratory infection (aerosol).

Human disease: Bolivian haemorrhagic fever - high fever, headaches, pain in abdomen and extremities, conjunctivitis, erythema on head and chest, petechial bleeding in the skin, mouth, gastrointestinal and urogenital tracts, 
neurological defects (palsy, epilepsy), hypovolemic shock; fatality rate 10$30 \%$; very long convalescence. Epidemics and sporadic cases have occurred in Bolivia since 1964 (discovery date), e.g. in 1994 or the latest in 2007 with 20 cases ( 3 fatal); in total, several thousand cases have been estimated to occur in Bolivia.

Bio-containment: BSL-4.

Diagnosis: ELISA (IgM and IgG), RT-PCR, immunohistochemistry (post mortem).

Treatment: immunne serum (immediately after infection), ribavirin in the early phase.

Prevention: vaccine (Junin, partially cross-protecting).

Geographical distribution: South America (Bolivia).

\section{Arenavirus Guanarito}

Source of infection (natural host range): short-tailed cane mouse Zygodontomys brevicauda (reservoir), Sigmodon alstoni.

Animal disease: unknown.

Transmission mode: alimentary, contact (percutaneous), aerogenic (in farmers).

Human disease: Venezuelan haemorrhagic fever with malaise, myalgia, anorexia, headache, back pain, dizziness, nausea, vomiting, prostration, petechiae, bleeding gums, later bleeding from mucous membranes, tremor, lethargy, convulsions, occasional coma and death.

Bio-containment: BSL-4.

Diagnosis: ELISA (IgM and $\operatorname{IgG})$, RT-PCR, immunohistochemistry (post mortem).

Treatment: immune plasma or ribavirin in the early phase.

Geographical distribution: South America (Venezuela).

\section{Arenaviruses Sabiá and Chapare}

Closely related viruses, but distinct species.

Source of infection (natural host range): rodents (reservoir).

Animal disease: unknown.

Transmission mode: contact, alimentary, aerogenic.

Human disease: haemorrhagic fever with a course similar to the other New World arenaviruses. First case of Sabiá virus disease (fatal) reported in Sao Paolo in 1990, and several laboratory infections have been also described. Chapare virus caused a small epidemic in Bolivia (2003/2004) with at least one fatal case.

Bio-containment: BSL-4.

Diagnosis: ELISA (IgM and IgG), RT-PCR, immunohistochemistry (post mortem).

Treatment: immune plasma or ribavirin in the early phase.

Geographical distribution: South America - Brazil (Sabiá) and Bolivia (Chapare). 


\section{Arenavirus Whitewater Arroyo}

Source of infection (natural host range): white-throated woodrat Neotoma albigula.

Animal disease: unknown.

Transmission mode: contact or aerosol.

Human disease: haemorrhagic fever (3 fatal human cases reported in California).

Bio-containment: BSL-2.

Diagnosis: ELISA (IgM and $\operatorname{IgG})$, RT-PCR, immunohistochemistry (post mortem).

Treatment: ribavirin in the acute phase.

Geographical distribution: Southwest USA.

\subsubsection{Family Filoviridae}

Filoviruses are characteristic for their long filamentous virions (under electron microscopy, some of them appear ring-like, others in the shape of the figure " 9 ", or even branching), $800-1000 \times 80 \mathrm{~nm}$ or longer, up to $14 \mu \mathrm{m}(!)$, enveloped, containing one molecule of ss(-) RNA sized $19 \mathrm{kbp}$.

\section{Marburgvirus}

Source of infection (natural host range): monkeys (Cercopithecus aethiops and C. ascanius), fruit bats (the reservoir - 2007 Gabon, Uganda, Kenya: detection of the virus RNA in 1-5\% of examined Rousettus aegyptiacus (Photo 5.28), several virus isolations from bat tissues, and IgG antibodies detected in 2-12\% bat individuals), insectivorous bats Rhinolophus eloquens, Miniopterus inflatus in DR Congo; man.

Animal disease: sometimes inapparent, but often fatal haemorrhagic disease in primates such as Cercopithecus aethiops or chimpanzee (up to 50\% fatality rate) and also at experimental infection of some other monkey species of the Old World. Asymptomatic course in fruit bats.

Transmission mode (Fig. 8.15): contact (primate saliva, blood and excreta; faeces of infected fruit bats: experimentally infected bats shed the virus for up to 3 weeks), iatrogenic (injections, blood transfusion), less often aerogenic; a high contagiosity - a number of nosocomial and several laboratory infections.

Human disease: Marburg haemorrhagic fever, one of the most fatal infectious diseases, with chills, severe headaches, myalgia, pharyngitis, nausea, abdominal pain, diarrhoea, extreme fatigue, maculopapular rash most prominent on the trunk (chest, back, abdomen), skin hypersensitivity, neurological disturbances, conjunctivitis, lymphadenitis, splenomegaly, leucopenia, thrombocytopenia, massive haemorrhages - bleeding from nose, ears and eyes, bloody faeces and bloody (black) vomites, severe cachexia, multiorgan dysfunction 
Fig. 8.15 The cycle of Marburg virus (drawing by Ivo Rudolf)

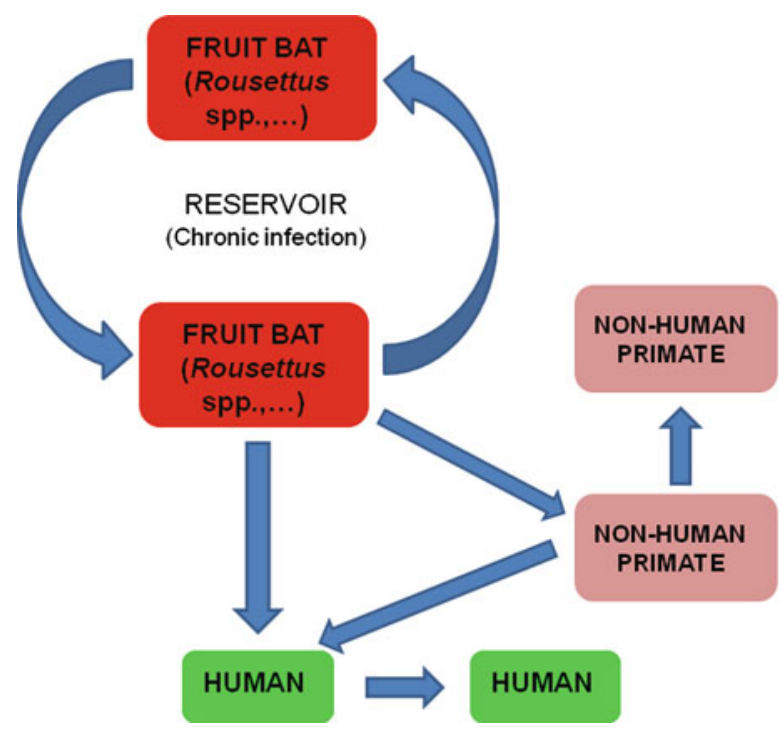

(liver etc.), hypovolemic shock and renal failure, delirium, the face looking as a mask; fatality rate 50-90\%. Long-term sequelae in many convalescents (chronic hepatitis, transverse myelitis, uveitis, parotid gland, orchitis, impotence in some men). Epidemics: 1967-1968 Germany (Marburg, Frankfurt) and Serbia (Beograd): marmosets Cercopithecus aethiops imported from Uganda - 31 laboratory personnel fell ill, 7 died; 1975 South Africa 3 cases in Australian tourists; 1980 and 1987 Kenya (Mt. Elgon) 3 tourists visiting the Kitum Cave died; 1999-2000 DR Congo (Durba: a gold mine) - 149 cases, 86\% of patients died; 2004-2005 north Angola: 252 ill persons, 90\% succumbed; 2007 Uganda: 10 miners working in a lead mine died; 2008, a Dutch tourist (visiting a cave with fruit bats in Uganda) died.

Bio-containment: BSL-4.

Diagnosis: isolation of the virus from the blood or other body fluids on Vero cells or in guinea pigs (a risky procedure), electron microscopy, RT-PCR; detection of $\operatorname{IgM}$ in the blood.

Treatment: therapy does not exist (ribavirin ineffective), only supportive treatment.

Geographical distribution: tropical Africa (Uganda, DR Congo, Zimbabwe).

\section{Ebolavirus}

Five genotypes: Sudan (1976), Zaire (1976), Côte d'Ivoire (1994), Bundibugyo (Uganda, 2007) and for man nonpathogenic Reston virus (1989: monkeys Macaca fascicularis imported in the USA from the Philippines).

Source of infection (natural host range): primates including man, bats. However, the reservoir are bats including fruit bats (experimental viraemia 
lasted up to 21 days in some species - Tadarida condylura, T. pumila, Epomophorus wahlbergi); hunted fruit bats can also be the source of infection. Recently, Ebolavirus RNA was detected in visceral organs of fruit bats Hypsignathus monstrosus, Epomops franquetti, and Myonycteris torquata.

Animal disease: usually inapparent except for chimpanzee (fatality rate up to $50 \%$ ), but experimental infection of monkeys often fatal. Reston virus is pathogenic to pig.

Transmission mode: contact and alimentary (saliva, blood and excretions of primates, hunter contacts with dead chimpanzees and monkeys and their meat; tree fruit contaminated with bat saliva and other excreta), iatrogenic (inoculation, transfusions), less often aerogenic; high contagiosity - nosocomial and laboratory infections occur quite frequently. Funeral rituals are also a common risk factor during the outbreaks.

Human disease: Ebola haemorrhagic fever, one of the most fatal human infectious diseases, with acute and severe headaches, pharyngitis, pains of muscles, sore back, abdominal pain, hiccups, severe diarrhoea, nausea, vomiting, extreme fatigue and weakness, rash over whole body, neurological and psychotic disturbances (disorientation etc.), conjunctivitis, leucopenia, thrombocytopenia, effective blocking of interferon production, systemic haemorrhages, bloody faeces and bloody vomites, cachexia, hypovolemic shock and renal failure, the face looking as a mask; fatality rate $50-80 \%$; a long-term convalescence. A number of mild or asymptomatic Ebolavirus infections have been observed in humans - in some enzootic areas (e.g., in Gabon) a relatively high proportion of population (about 10\%) has antibodies to ebolavirus Zaire without being clinically ill before. Epidemics in the years 1976, 1979 and 1995 in Sudan and Zaire caused a total of 818 cases with a very high mortality: e.g., during the 1995 outbreak in Zaire $79 \%$ and in Sudan 53\%. Other epidemic in Gabon 1994-1996 had 80 cases, 2000-2001 outbreak in Uganda with 428 cases (52\% fatality rate), 2002-2004 central Africa (Gabon, DR Congo) 428 cases (fatality rate 78\%). During the years 1976-2000 a total of 1,524 cases of Ebola fever were recorded, and 1,010 of the patients died. A more recent outbreak was reported from DR Congo (Luebo, Kasai province) in 2007: 372 cases (186 patients died); this outbreak was linked to a masssive migration of fruit bats hunted by villagers at the bat daily communal roosts situated often on palm trees close to the village. A large proportion of the human population living in forested areas of Gabon has both humoral and cellular immunity to Zaire ebolavirus. A new genotype, Bundibugyo virus, was the cause of 116 confirmed or probable haemorrhagic cases in western Uganda, 2007-2008, with a fatality rate of 34\%). The Côte d'Ivoire virus caused only one non-fatal haemorrhagic human case.

Bio-containment: BSL-4.

Diagnosis: isolation of the virus from the blood or other body fluids on Vero cells or in guinea pigs (extreme Biohazard level: BSL-4), electron microscopy, RT-PCR; detection of IgM in the blood (ELISA); immunohistochemistry (post-mortem). 
Treatment: therapy does not exist, ribavirin ineffective; only supportive care.

Prevention: avoiding contact with infected humans or non-human primates and fruit bats (and their habitats like roosting sites in caves) infected with the virus.

Geographical distribution: tropical Africa (Zaire = DR Congo (Photos 5.29, 5.30, 5.52), Gabon, Sudan, Uganda, Ivory Coast, Cameroon); the Philippines (Reston virus).

\subsubsection{Family Orthomyxoviridae}

\section{(**) Orthomyxovirus Influenza A}

Virions are spherical to pleomorphic 80-120 nm, enveloped, with 8 segments of ss(-)RNA sized 10-15 kbp and with two surface antigens (glycoproteins): haemagglutinin (16 $\mathrm{H}$ subtypes) and neuraminidase (nine $\mathrm{N}$ subtypes). All these antigenic subtypes have been detected in birds, while in mammals only some of them: for instance in humans they have been found only H1,2,3,5,7,9 and N1,2,7. Changes and evolution of influenza A viruses: antigenic "drift" (restricted mutations in $\mathrm{H})$. . . antigenic "shift" (changes in $\mathrm{H}$ and $\mathrm{N}$ associated with recombinations or reassortement of individual subtypes in vertebrates (duck, pig) leading to appearance of new subtypes).

Source of infection (natural host range): man; in zoonotic influenza pig, horse, and fowl. Free-living waterfowl, gulls and shorebirds are the main reservoir of influenza A viruses in nature. Some wild anseriforms (swans, geese, ducks) also participate in the spread of highly pathogenic avian influenza (HPAI) viruses.

Animal disease: inapparent (in a majority of waterbirds) or fever, sinusitis, bronchopneumonia, oedema of the face and neck, cyanosis - e.g., "fowl plague" in hens due to HPAI virus subtypes $\mathrm{H} 5$ and H7, with a fatality rate 70-100\%; these subtypes may cause clinical disease also in some terrestrial and aquatic mammals.

Transmission mode: aerogenic (a droplet infection), also by conjunctivae and contact. In the zoonotic HPAI H5N1 virus is the highest risk of human infection associated with processing of fowl (killing, removal of feather, cutting into portions), and in Asian animal markets with living fowl if it is infected. Human-to-human transmission of HPAI viruses has been absent or very rare.

Human disease: influenza (an anthroponosis), often complicated with pneumonia, with the fatality rate of approximately $1 \%$. The known anthroponotic influenza pandemics occurred in 1918/19 ('Spain flu', H1N1, which killed about 21 million persons but some sources give as many as 40 million victims); 1957/58 ('Asian flu', H2N2: >1 million victims; 1968/70 ('Hongkong flu', H3N2: at least 700,000 deaths; 1977 ('Russian flu', H1N1); and the recent pandemic of 'swine (Mexican) influenza' (H1N1) has encompassed 
213 countries and caused 18,449 deaths (as of 1 August 2010). Except for the recent outbreak, all pandemic influenza A strains had in its genome inserted characteristic fragments of avian influenza viruses; they therefore emerged as reassortants of avian and mammalian influenza strains during co-infection of a susceptible mammal host (usually domestic pig as a 'mixing vessel'). However, sporadical zoonotic influenza infections of humans with equine, swine and avian strains have been reported, usually without a further spread in human population. For instance in Wisconsin (1990) several pig breeders became ill, and one pregnant women died. This so-called swine influenza caused about 50 laboratory-confirmed cases (7 patients died) worldwide up to 2008. Another zoonotic problem is avian influenza; first cases of it were described in Hongkong (1997), where 18 persons were infected from chickens with the HPAI virus type H5N1 from chickens, and 6 of the patients died (one million hens had to be put down). In 2003, one veterinarian died after being infected with HPAI virus type H7N7 in the Netherlands (30 million chickens were then killed, and during this action 89 persons get fever with severe conjunctivitis). Further cases of infection with HPAI virus H5N1 acquired directly from chickens were reported in 2003-2010: from a total of 504 laboratory-confirmed human cases in 15 countries (reported to the WHO as of August 12, 2010), the highest incidence was in Indonesia (168 cases), Vietnam (119), and Egypt (111) - 299 patients died (case fatality rate of $59 \%$ ). The fatality rates of $\mathrm{H} 5 \mathrm{~N} 1$ avian influenza in humans have varied from $32 \%$ (Egypt) to 50\% (Vietnam), 67\% (China), 68\% (Thailand), and 83\% (Indonesia). During the same period, at least 200 million fowls were culled. Close contacts of humans with domestic ducks, chickens and pigs (common dwellings) in southeastern Asia present a permanent epidemiological risk into the future.

Bio-containment: BSL-2 (but HPAI H5N1: BSL-2/3).

Diagnosis: isolation and cultivation in chick embryos or on cell cultures, detection of antigen or RNA in the samples, serology (HIT).

Treatment: antivirotics amantadin, rimantadin, zanamivir (Relenza), oseltamivir (Tamiflu) might moderate the clinical course, but resistant strains of influenza A have already appeared.

Prevention: vaccine (human and fowl), culling of infected animal breeds.

Geographical distribution: worldwide; most strains of zoonotic influenza originate in Southeast Asia.

\section{Thogotoviruses Thogoto and Dhori}

Virions are spherical, 80-120 nm, enveloped, contain ss(-)RNA arranged in 6 (Thogoto) or 7 (Dhori) segments with a total size of $10 \mathrm{kbp}$, and one surface glycoprotein.

Source of infection (natural host range): cattle, camel.

Animal disease: inapparent course. 
Transmission mode: metastriate ticks Rhipicephalus and Amblyomma spp.

Human disease: severe fever, the course can be complicated occasionally.

Bio-containment: BSL-3.

Geographical distribution: Africa, southern Europe (Sicily, Portugal), Asia, southern Russia.

\subsubsection{Family Paramyxoviridae}

\section{Avulavirus Newcastle Disease (Avian Paramyxovirus 1)}

Virions are spherical or pleomorphic, $150 \mathrm{~nm}$ or more, with one molecule ss(-)RNA sized $15 \mathrm{kbp}$.

Source of infection (natural host range): domestic, wild and pet birds.

Animal disease: Newcastle disease (ND) of fowl and other birds, e.g. wild cormorants in North America, with the fatality rate of $10-90 \%$.

Transmission mode: contact, aerogenic (workers in fowl farms, laboratory accidents).

Human disease: sporadic - painful conjunctivitis, regional lymphadenitis, pharyngitis, bronchitis, atypical pneumonia, affection of the CNS.

Bio-containment: BSL-2/3.

Diagnosis: isolation of the virus from ocular irrigations or from the blood and urine, serology (VNT, ELISA, HIT).

Prevention: fowl vaccination.

Geographical distribution: worldwide.

\section{Henipavirus Hendra}

Also called equine morbillivirus, or bat paramyxovirus. Virions are spherical or pleomorphic, about $150 \mathrm{~nm}, 1$ molecule ss(-)RNA sized $15 \mathrm{kbp}$. The virus is distantly related to distemper morbillivirus.

Source of infection (natural host range): horse; fruit bats (reservoir: Pteropus alecto, $P$. poliocephalus, $P$. scapulatus, $P$. conspicillatus).

Animal disease: fruit bats asymptomatic; in horses fever and bronchopneumonia, haemorrhage, encephalitis; fatality rate high, $65 \%$. The disease has been known since 1994/1995, when 23 horse cases (16 died) were recorded on 3 places of Queensland, 13 of them in Hendra (a suburb of Brisbane). Pigs are susceptible to Hendra virus in experiment and develop fever, depression, respiratory and CNS affection signs.

Transmission mode (Fig. 8.16): contact - exposition to infected horses body fluids.

Human disease: affection of the respiratory tract (haemorrhagic pulmonary oedema), headaches, myalgia, encephalitis. Three cases (two fatal) were described in owners and trainers of 22 ill horses (14 died) from Hendra 
Fig. 8.16 Circulation of Hendra virus (drawing by Ivo Rudolf)

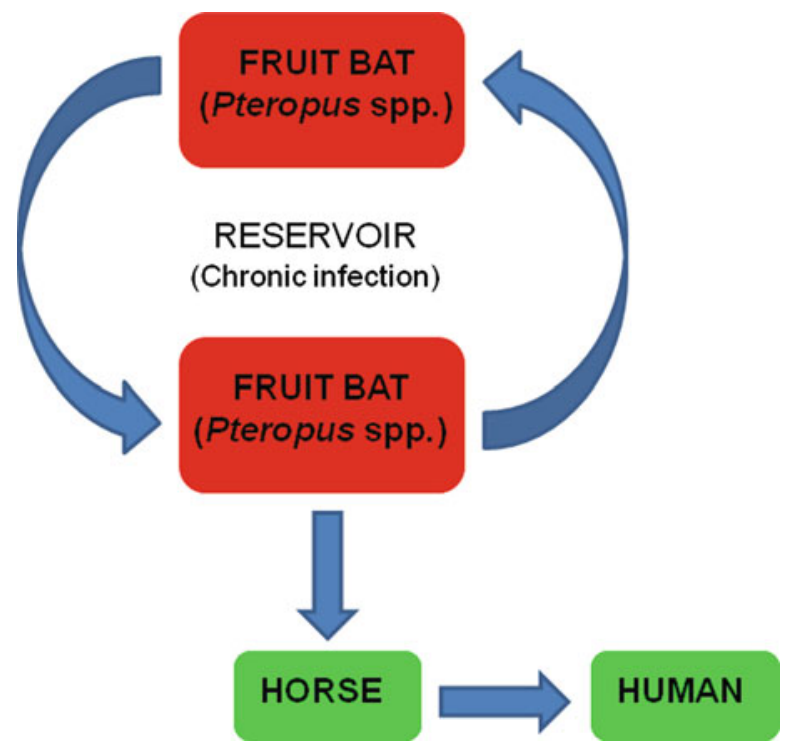

and Mackay in 1994, and similar 7 cases of horse owners and attending veterinarians (two were fatal) were documented in 2008-2009.

Bio-containment: BSL-4.

Diagnosis: isolation (urine, CSF, throat swab, nasal secretions) - risky procedure, RT-PCR, ELISA, VNT, IFA, immunohistochemistry.

Geographical distribution: Australia (Queensland), Papua New Guinea.

\section{Henipavirus Nipah}

Antigenically closely related to Hendra virus, the difference in nucleotide sequence of the genome is only $10-20 \%$.

Source of infection (natural host range): pig (cat, dog, goat, man); the reservoir are fruit bats - Pteropus hypomelanus, P. giganteus, P. lylei and $P$. vampyrus (the virus was isolated from their guano in Thailand, Cambodia and Indonesia, and antibodies have been found in up to $30 \%$ of the fruit bats).

Animal disease: barking pig disease with fever, bronchopneumonia, secretion of mucus with blood from nasal and oral cavities, necrotic vasculitis, often encephalitis, pareses of hind limbs, and death; fatal rate nearly $100 \%$ in pigs. Asymptomatic in fruit bats. Bats contaminate fruit which have been used as pig feed. Experimental infection: fatal pneumonia in horse, cat, dog and guinea pig, and squirrel monkey Saimiri sciureus.

Transmission mode (Fig. 8.17): contact, food-borne, aerogenic (high contagiosity; in contrast to Hendra virus, this henipavirus is excreted in urine and by coughing of infected animals), and alimentary (consumption of raw 
Fig. 8.17 Circulation of Nipah virus (drawing by Ivo Rudolf)

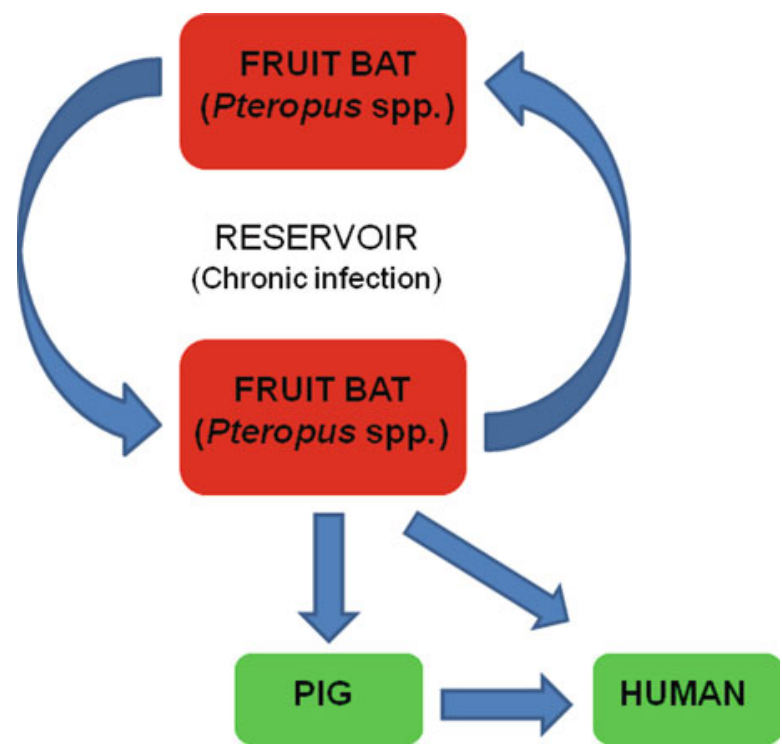

date palm sap - Bangladesh). Occupational disease: butchers (Singapore). In 2007, man-to-man transmission was documented for the first time - in Bangladesh (where more than half of the cases were nosocomial) and India.

Human disease: fever, headache, bronchopneumonia, vasculitis and thromboses, decreased consciousness, encephalitis; high fatality rate, $40 \%$. Epidemics: 1998-1999 Malaysia 265 cases (41\% fatal) and Singapore 11 cases (1 fatal); 2001-2007 Bangladesh several outbreaks with 192 cases in total and fatality rate of $69 \%$; 3 additional fatal cases here (Faridpur district) have been reported in 2010.

Bio-containment: BSL-4.

Diagnosis: isolation (urine, CSF, throat swab, nasal secretions) - risky procedure, RT-PCR, ELISA, VNT, IFA, immunohistochemistry.

Prevention: liquidation of pig breeds affected (in Malaysia, 1999, 900,000 domestic pigs were slaughtered originating from 900 farms).

Geographical distribution: Malaysia, Singapore (introduced in 1999 with import of pigs from Malaysia), Thailand, Cambodia, Indonesia, India, Bangladesh, southern China. According to some serosurveys, similar virus also occurs on Madagascar.

\section{Henipavirus Menangle}

Antigenically related to Nipah virus. The virus was first identified in a piggery in Menangle near Sydney (Australia) in 1997 where they experienced a high number of pig stillbirths and deformities during farrowing.

Source of infection (natural host range): pig, man; the reservoir are fruit bats Pteropus conspicillatus and Aethalops alecto. 
Animal disease: in pigs abortions and teratogenic defects; mummified and stillborn piglets with severe degeneration of the brain and spinal cord (almost absent in some piglets), arthrogryposis, brachygnathia, and occasionally fibrinous body cavity effusions and pulmonary hypoplasia, occasionally nonsuppurative myocarditis. Asymptomatic infection in fruit bats.

Transmission mode: contact (wounds on hands and forearms) with bodily fluids from infected animals (blood and possibly foetal matter-e.g., amniotic fluid); aerogenic.

Human disease: a febrile illness with chills, malaise, headache, and a measleslike rash; only two human cases have been described in Australia (19971998).

Bio-containment: BSL-3.

Diagnosis: isolation (urine, CSF, throat swab, nasal secretions) - risky procedure, RT-PCR, ELISA, VNT, IFA, immunohistochemistry.

Geographical distribution: Australia.

\subsubsection{Family Bornaviridae}

Spherical virions (85-125 nm) with one molecule of ss(-)RNA, unsegmented, $9 \mathrm{kbp}$ in size.

\section{Bornavirus}

Remark: many virologists do not regard that this is a zoonotic agent.

Source of infection (natural host range): horse; sheep, goat, cattle, rabbit, and other mammals (unclear). The natural virus reservoir is unknown at present (rodents?).

Animal disease: Borna disease, a fatal meningoencephalitis of horse and sheep (first epizootic described in Germany, 1885).

Transmission mode: contact with animal excreta (saliva, tears, nasal discharge), possibly also aerogenic and alimentary. Tenacity of the virus in the dry state is exceptionally long (1-3 years).

Human disease: neuropsychiatric disorders not sufficiently characterised, possibly also lymphocytic meningoencephalitis; bornavirus antibodies but also RNA were found in CSF and peripheral blood leucocytes of some psychiatric or immunocompromised patients in several studies.

Bio-containment: BSL-2.

Diagnosis: isolation in cell cultures; intracerebral inoculation of rabbit (fatal).

Geographical distribution: Germany, Austria, Switzerland, France; infrequently it occurs in Japan and USA.

\subsubsection{Family Coronaviridae}

\section{Coronavirus SARS}

Also called severe acute respiratory syndrome-associated coronavirus (SARS$\mathrm{CoV})$. Virions are spherical to oval (120-160 nm), enveloped, with a molecule of ss(+)RNA sized 28-31 kbp. 
Source of infection (natural host range): civet Paguma larvata, raccoon-like dog (Nyctereutes procyonoides), Chinese ferret-badger (Melogale moschata) and other medium-sized carnivores on South-Asian animal markets; man. However, probable reservoir are bats (SARS-like coronaviruses have been isolated from Rhinolophus spp., Miniopterus magnater, and Pipistrellus spp.).

Animal disease: asymptomatic infection.

Transmission mode: aerosol and contact. Man-to-man transmission is common. The virus has a considerable tenacity in environment - it survives up to 1 week on surface of various utensils, or in faeces.

Human disease: severe acute respiratory syndrome (SARS), which also affects gastrointestinal tract, and has high fatality rate (40-50\%). SARS in children has a less severe course than in adults. The proportion of asymptomatic infections in humans is low. First outbreak was reported from the Chinese province Guangdong in November 2002; the epidemic then jumped to Hongkong (hotel Metropol etc.) and to many other localities and countries over the world, becoming a dreadful pandemic. In the period to May 2003, a total of 20,791 patients were reported (9,662 of the cases were fatal) from 30 countries in 5 continents. One of the victims was also epidemiologist Carlo Urbani who put WHO on the alert at beginning of the epidemic.

Bio-containment: BSL-3.

Diagnosis: RT-PCR, isolation of the virus, serology (ELISA).

Geographical distribution: China, Hongkong, Taiwan, Singapore; exported on airplanes also to Vietnam, Canada (Toronto), USA and 22 other countries.

\subsubsection{Family Picornaviridae}

Virions are spherical $(30 \mathrm{~nm})$, not enveloped, with one molecule ss(+)RNA sized 7-8 kbp.

\section{Cardiovirus Encephalomyocarditis}

Some virologists doubt that EMC virus is zoonotic in that an antigenically very similar Saffold cardiovirus is anthroponotic.

Source of infection (natural host range): rodents (reservoir - but it is uncertain whether EMC virus from rodents is transmissible to humans), pig and other domestic mammals, lagomorphs, non-human primates.

Animal disease: encephalomyocarditis (EMC) or inapparent course; sporadic cases and outbreaks of myocarditis, encephalitis, and abortions in pigs.

Transmission mode: aerogenic.

Human disease: an uncommon disease with fever, headache, malaise, sweats, chills, myalgia, pallor, nausea, vomiting, and abdominal pain, weight loss, arthralgia, retro-ocular pain, photophobia, sometimes meningitis to encephalomyelitis; no mortality reported.

Bio-containment: BSL-2.

Geographical distribution: worldwide, but sporadic. 


\section{Parechovirus Ljungan}

The virus is related to cardioviruses, it was isolated and named after a river in central Sweden in 1998. Scandinavian and American strains differ, and there are known three genotypes of Ljungan virus at present.

Source of infection (natural host range): rodents, especially voles (Myodes glareolus, Microtus montanus) - reservoir; also detected in Apodemus flavicollis.

Animal disease: diabetes mellitus (usually during overpopulation; the virus attacks pancreas) and myocarditis in Myodes glareolus, or inapparent course.

Transmission mode: probably aerogenic.

Human disease: intrauterine foetal death (the virus infects the CNS of embryo) -2 cases have been described (Sweden, 2007, and USA 2008). Ljungan virus is suspect of causing also sudden infant death syndrome, CNS embryonal malformations (hydrocephalus, anencephaly - which has been confirmed in mouse), and it has been detected in adult patients with diabetes and myocarditis.

Bio-containment: BSL-2.

Geographical distribution: Sweden, Denmark, Italy, USA, but probably worldwide.

\subsubsection{Family Caliciviridae}

Virions are spherical (30-40 nm), not enveloped, with one molecule ss(+)RNA sized $8 \mathrm{kbp}$.

\section{Swine Norovirus}

Source of infection (natural host range): domestic pig (but only the strains of the genomic group II are zoonotic); oysters.

Animal disease: gastroenteritis, or asymptomatic infection.

Transmission mode: alimentary (pork, oysters, other seafood etc.).

Human disease: gastroenteritis. Simultaneous outbreaks occurred in UK, Norway, France, Sweden, and Denmark after consumption of contaminated oysters in 2009 and 2010 (334 cases in 65 clusters).

Bio-containment: BSL-2.

Geographical distribution: probably worldwide.

\subsubsection{Family Hepeviridae}

\section{(**) Hepevirus Hepatitis E}

Virions spherical $(30 \mathrm{~nm})$, not enveloped, with one molecule ss(+)RNA sized 7.2 $\mathrm{kbp}$; antigenically uniform, but four genotypes (types I and II are anthroponotic, while the types III and IV are zoonotic). 
Source of infection (natural host range): domestic pig, wild boar (reservoir), probably also red deer and rodents (brown rat, black rat); fowl, wild mongoose, horse, primates including man; shellfish.

Animal disease: inapparent course (piglets 2-3 months old) or gastroenteritis. Experimental infection successful in pig, monkey, sheep and rat.

Transmission mode: alimentary (food-borne: raw or undercooked swine liver and intestine, deer liver meat contaminated with the virus; or water-borne: drinking of water contaminated with contaminated sewage disposal); personto-person transmission (among household members during outbreaks); parenteral (blood transfusion during a viraemic period), mother-to-child transmission (scarcely documented).

Human disease: hepatitis E - after a long incubation period (15-60 d.,

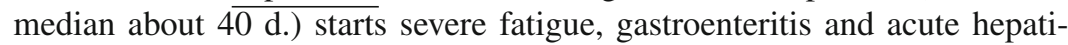
tis (increased levels of liver enzymes ALT and AST); fatality rate $<1 \%$. The infection is dangerous for pregnant women (with an up to $25 \%$ fatality rate). Big epidemics have been repeatedly reported in so-called endemic areas (and caused by anthroponotic strains): 1955 India 30,000 cases; 1976 Myanmar 20,000; 1978 Kashmir 52,000 (1,500 patients died); 1986 China 100,000 and Somalia 11,000; 1989 Mexico 4,000. The zoonotic hepatitis E, which occurs mainly in so-called non-endemic areas (Europe, North America, Japan), is probably an underdiagnosed disease (a number of subclinical cases occur). For instance, 116 cases of hepatitis E were diagnosed in Hungary from 2001 to 2006.

Bio-containment: BSL-2.

Diagnosis: isolation on cell cultures, IF; serology - VNT, ELISA (IgM antiHEV).

Geographical distribution: zoonotic genotype III probably worldwide, it has been demonstrated in Japan, Korea, Indonesia, China, France, Great Britain, Sweden, Spain, the Netherlands, Czechland, Hungary, Greece, USA and Africa, while the other zoonotic genotype IV is restricted to India and East Asia. Anthroponotic genotype I extensively circulates in Asia (including India, Pakistan, Nepal, Bangladesh, China, Kyrgyzstan, and Uzbekistan) and Africa (including Egypt, Algeria, Morocco, Namibia, Sudan and Chad); genotype II occurs in Mexico and in some African countries (Nigeria, Namibia, Chad, and Sudan).

\subsubsection{Family Retroviridae}

\section{**Lentivirus HIV-1, HIV-2}

Virions spherical $(110 \mathrm{~nm})$, enveloped, contain 2 identical molecules (dimer) ss(+)RNA, reverse transcriptase, and 2 surface proteins.

Source of infection (natural host range): man. HIV-1 ("human immunodeficiency virus") evolved from SIV ("simian immunodeficiency virus") of 
chimpanzee Pan troglodytes troglodytes (reservoir), and HIV-2 from SIV of sooty mangabey Cercocebus torquatus. HIV-1 and HIV-2 have therefore zoonotic origin, connected with crossing the species barrier (a host-jumping event).

Animal disease: decreased immunity with a milder course than is AIDS in humans.

Transmission mode: as the originating zoonosis by contact with the blood of killed wild primates ("cut-hunter hypothesis"); as anthroponosis transmitted sexually, by inoculation or transfusion (blood), transplantation of organs, and vertically from mother to child.

Human disease: acquired immune deficiency syndrome (AIDS) - first infections demonstrated sporadically already before 1959 (DR Congo - antibodies); start of AIDS epidemics were revealed in homosexual communities of New York and California in 1981, the number of patients increased continuously resulting in a pandemic: in the year 2001, 40 million persons were infected with HIV or had symptoms of AIDS, of this number 28 million in sub-Saharan Africa (local HIV prevalence rate was in the age 15-25 years $10 \%$, in adults $20 \%$ ) and 8 million in southeast Asia; 3 million patients succumbed to AIDS worldwide in the same year.

Bio-containment: BSL-2.

Treatment: zidovudin, Retrovir, Combivir.

Prevention: safe sexual behaviour, use of disposable injection syringes and needles in drug addicts.

Geographical distribution: worldwide.

\subsubsection{Family Herpesviridae}

Virions approximately spherical (100-230 nm), enveloped, contain a linear molecule dsDNA 125-240 kbp.

\section{Varicellovirus Herpesvirus Suis 1 (pseudorabies virus, suid herpesvirus 1)}

Source of infection (natural host range): pig (reservoir), ruminants, rodents, dog, cat.

Animal disease: pseudorabies (morbus Aujeszky) - encephalomyelitis with bronchopneumonia, fatal for piglets; in adult pigs the course is inapparent.

Transmission mode: direct contact, percutaneous (biting).

Human disease: nettle-rash, salivation, pharyngitis, affection of the CNS. However, the possibility of a zoonotic infection of man with this virus is unclear and doubted by many virologists.

Bio-containment: BSL-2.

Diagnosis: isolation in cell cultures, IF; VNT, ELISA.

Geographical distribution: worldwide. 


\section{Macacine Simplexvirus 1 (Herpesvirus B, Monkey B virus)}

Source of infection (natural host range): monkeys of the Old World (reservoir Macaca spp., mainly M. mulatta as well as M. fascicularis, but also M. fuscata, $M$. arctoides, $M$. cyclopsis and $M$. radiate): they excrete the virus with saliva and other secrets.

Animal disease: inapparent course or stomatitis (small vesicles on tongue and mucosa), rarely encephalomyelitis.

Transmission mode: contact - percutaneous (biting, scratching), also aerogenic; most commonly after a stress (caused e.g. by transport of the animals); there were reported also several laboratory infections during the work with monkey cell cultures.

Human disease: simian herpes B (herpes simiae) - vesicles, lymphadenitis, severe encephalomyelitis with the fatality rate of $80 \%$. Up to now a total of 40 cases have been reported. Risk groups: people getting into close contact with macaques (attendants, hunters, etc.).

Bio-containment: BSL-3.

Diagnosis: isolation on cell cultures, IF; VNT, ELISA.

Treatment: acyclovir (Zovirax).

Geographical distribution: southeastern Asia (also temples in Nepal and India), North Africa; secondarily other regions by transport of monkeys.

\subsubsection{Family Poxviridae}

\section{Orthopoxvirus simiae (Monkeypox Virus)}

Large subspherical or moderately pleomorphic virions (about $250 \times 200 \mathrm{~nm}$ ), enveloped, containing one linear molecule dsDNA sized 170-250 kbp.

Source of infection (natural host range): monkeys of the Old World, rodents (African rope squirrels Funisciurus congicus, F. anerythrus, red-legged sun squirrel Heliosciurus rufobrachium, and rats Cricetomys emini, $C$. gambianus), as well as some other wild mammals and domestic pig.

Animal disease: pox.

Transmission mode: contact with wild animals (especially in hunters); the man-to-man transmission is relatively rare, but after 1995 the proportion of secondary man-to-man infection has obviously increased.

Human disease: monkeypox, similar to smallpox, with fever, hyperhidrosis, headaches, myalgia, backaches, extreme fatigue, rash, lymphadenitis (the latter symptom differentiates both diseases); fatality rate can attain $10 \%$ (reports show 4-33\%) - most lethal is monkeypox for small children up to 4 years old. Monkeypox in humans was described in 1970, and to 1999 nearly 1,000 cases (40 fatal) were reported in Africa: e.g., 398 cases in DR Congo between 1981 and 1986, an outbreak in the same country in 1996/1997 (88 cases, fatality rate 4\%), and a total of 51 laboratory-confirmed cases between 2001 and 
2004. In 2003, monkeypox was imported with wild rodents of several genera (e.g. Cricetomys, Graphiurus) from Ghana to Wisconsin (USA) where several captive American prairie dogs (Cynomys ludovicianus) were infected and unintentionally distributed to other US states; 35 attending persons (12 veterinarians and several animal shop assistants) acquired the infection as well, and the number of humans infected with monkeypox increased then to 82 in USA.

Bio-containment: BSL-3.

Diagnosis (valid also for other poxvirus infections): symptomatology; histopathology and electron microscopy of the vesicular lesions, IF, and isolation (on chick embryos or cell cultures Vero, LLC-MK2) from skin lesions; serology (HIT, ELISA, RDPA).

Treatment: cidofovir.

Prevention: vaccine against smallpox protects against monkeypox.

Geographical distribution: tropical forests in central and western Africa (DR Congo, Cameroon, Ghana, Gabon), Southeast Asia.

\section{Orthopoxvirus bovis (Cowpox Virus)}

By passaging this virus originated the vaccinia virus $O$. officinalis, [Lat. vacca $=$ cow], used for vaccination against smallpox (variola, i.e. human pox). [This virus is also used for preparation of recombinant vaccines against different viruses, for instance the oral vaccine against fox rabies].

Source of infection (natural host range): cattle, camel, rabbit; brown rat including pet rats (Rattus norvegicus), and other rodents (reservoir), cat.

Animal disease: cowpox, variola vaccina. Zoo and circus mammals (elephants, rhinoceros, felids - often fatal), wild rodents.

Transmission mode: direct contact; infrequently also aerogenic and alimentary (risk - pet rodents).

Human disease: cowpox (uncommon) - papulae and pustulae on the skin, lymphatic changes on the hands, lesions on eyelids (the Netherlands 2002); exceptionally a generalized illness. Several human cases of cowpox have been reported since 2008 as acquired from pet rats in Germany and France. Occupational disease in farmers, milkers and their close contacts, also in zoo and circus animal attendants.

Bio-containment: BSL-2.

Geographical distribution: worldwide.

\section{Parapoxvirus bovis 1 (Bovine Papular Stomatitis Virus)}

Virions of parapoxviruses are oval, $220-300 \times 140-170 \mathrm{~nm}$. Size of DNA is 130-150 kbp.

Source of infection (natural host range): cattle.

Animal disease: papular stomatitis (bovine pustular stomatitis) - papulae and pustulae on the muzzle and in oral cavity. 
Transmission mode: direct contact (percutaneous).

Human disease: not commonly, bovine papular dermatitis - red-violet papulae and pustulae on fingers and forearm, the course is protracted.

Bio-containment: BSL-2.

Diagnosis: symptoms; isolation on cell cultures, electron microscopy of the lesion samples.

Geographical distribution: North America, Europe, Africa, Australia.

\section{Parapoxvirus bovis 2 (Pseudocowpox Virus)}

Source of infection (natural host range): cattle, sheep, pig.

Animal disease: paravaccine (pseudocowpox) - lesions on the udder of cows (papulae to pustulae).

Transmission mode: direct contact (percutaneously).

Human disease: milkers' nod(ul)es, paravaccinia - a disease milder than cowpox: small painful not suppurative papulae and nodules on the hands and forearms for up to 6 weeks.

Bio-containment: BSL-2.

Diagnosis: isolation on cell cultures, electron microscopy.

Geographical distribution: Europe, North America.

Parapoxvirus ovis (Orf parapoxvirus)

Source of infection (natural host range): sheep, goat.

Animal disease: contagious ecthyma, “orf" - pustulae and papulae on the face and udder.

Transmission mode: direct contact (percutaneous); the virus is resistant in the milieu (in scabs and crusts it can survive for years).

Human disease: contagious pustular dermatitis - painful dermatitis (papulae to pustulae) on fingers and arms and in the oral cavity, the course is protracted. The disease is rare, it occurs in sheep breeders and among butchers.

Bio-containment: BSL-2.

Diagnosis: electron microscopy of the pus from pustules and scabs.

Geographical distribution: worldwide.

\section{Yatapoxvirus tanapox (Tanapoxvirus)}

Virions $200 \times 300 \mathrm{~nm}$, DNA size is $145 \mathrm{kbp}$.

Source of infection (natural host range): monkeys (possibly also other wild and domestic animals).

Animal disease: papulae on the face.

Transmission mode: mosquitoes (Mansonia) - a mechanical transmission.

Human disease: fever with headaches and 1-2 pustular skin lesions for a period of up to 6 weeks.

Bio-containment: BSL-2. 
Diagnosis: electron microscopy, and virus isolation from skin lesions.

Geographical distribution: tropical Africa (Kenya).

\subsection{Bacteria}

\subsubsection{Family Chlamydiaceae [Order Chlamydiales, Class Chlamydiae]}

Members of the order Chlamydiales are very small Gram-negative spherical bacteria with a diameter of $0.3-0.5 \mu \mathrm{m}$, obligate intracellular parasites preferentially of the mucosal epithelium. They do have solid cell wall but without muramic acid (in contrast to other bacteria peptidoglycan between the wall and plasmatic membrane is absent). They do not posses their own energetic metabolism and depend on the ATP system of the host cell (they are therefore called "energetic parasites"); thanks to this adaption, their genome is markedly reduced. The peculiar life cycle of chlamydiae differs greatly from that of other bacteria, and involves small extracellular non-metabolising but infectious elementary bodies $(0.3-0.5 \mu \mathrm{m})$ that produce in the infected cell bigger and non-infectious reticulate bodies (also called "initial bodies", $0.7-1.1 \mu \mathrm{m}$ ); those divide by binary fission and form new elementary bodies which are able to survive extracellularly and also infect new hosts. Cell inclusions visible microscopically in chlamydial infections are, in fact, conglomerates (colonies) of replicating reticulate bodies together with the daughter elementary bodies. Finally, the infected cells crack or the bodies escape by exocytosis. Chlamydiae are well stained for microscopy according to Macchiavelli (initial bodies are stained blue, and reticulate bodies red) or Giemsa (blue and purple, respectively). The tenacity of chlamydiae in the milieu is very limited, especially for reticulate bodies (however, avian strains of Chlamydophila psittaci can survive in bird droppings for a long time). Chlamydiae are sensitive to diethyl ether because $50 \%$ of the cell wall content is formed by lipids, and are readily inactivated by certain antibiotics, largely those that inhibit proteosynthesis (chloramphenicol or tetracycline). The only typical zoonotic species among chlamydiae is Chlamydophila psittaci, while two other species cause anthroponotic infections: Chlamydophila pneumoniae causes respiratory infections (possibly also atherosclerosis and coronary disease), and Chlamydia trachomatis trachoma, but its serotypes $\mathrm{D}$ to $\mathrm{K}$ very frequent sexually transmissible infections of the human urogenital tract (and proctitis), and serotype $\mathrm{L}$ is the agent of lymphogranuloma venereum. In contrast to rickettsiae, chlamydiae are not transmitted by arthropod vectors.

\section{Chlamydophila psittaci}

A heterogenous species with a number of genotypes (A to F) differentiated according to the ompA gene (expressing outer membrane protein).

Source of infection (natural host range): domestic, wild and exotic (pet) birds in psittacines genotype A - psittacosis; in other birds ornithosis: genotype 
C prevails in anseriforms; genotype B in fowl ((hens, turkeys), feral pigeons (but also the genotype E, at least 500 human cases reported) and passerines; further gulls, egretts etc. - the disease was observed in 230 avian species belonging to 10 orders).

Animal disease: usually latent, sometimes subclinical or manifest - ornithosis of young fowl and pigeons, especially under stress conditions, with fluffed feathers, conjunctivitis (closed eyes), rhinitis, pneumonia, anorexia, lethargy, diarrhoea, pericarditis and enteritis, at a high fatality rate.

Transmission mode: aerogenic (inhalation of dust containing infected avian droppings); often occupational infectious disease (breeders, fowl farmers, butchers). Tenacity of the elementary bodies is relatively high.

Human disease: ornithosis/psittacosis ("parrot disease") - clinical symptoms are highly variable, from mild flu-like symptoms to severe pneumonia or systemic disease; the pneumonia is atypical (radiologic findings are substantially more pronounced than those physical - a nonproductive cough) with high prolonged fever that reveals relapses, headaches, myalgia and arthralgia, exhaustion, sometimes endocarditis, hepatosplenomegaly, encephalitis; fatality rate used to be high (e.g. it was $20 \%$ during the outbreaks transmitted by birds imported from Argentina in 1929/1930, but as high as $80 \%$ in pregnant women), today is only about $1 \%$. The highest incidence rate of psittacosis is reported from Australia, UK, Denmark, Sweden and the Netherlands.

Bio-containment: BSL-2/3.

Diagnosis: anamnesis (contact with ill birds); serology (CFT, ELISA - there might occur cross-reactions with $\mathrm{Q}$ fever); isolation on chick embryo and McCoy cells, PCR, detection by microscopy of chlamydiae in sputum samples or in inoculated cells using IF or staining (Giemsa, Macchiavelli, Giménez, Lugol's iodine); PCR.

Treatment: doxycycline, tetracycline (adults), erythromycin (children, 3 weeks).

Geographical distribution: worldwide.

\section{Chlamydophila abortus}

Source of infection: sheep, goat, cattle; occasionally pig, horse, deer.

Animal disease: ovine enzootic abortion; in cattle, abortion, stillbirth, mastitis, metritis, epididymitis, orchitis, pneumonia, and occasionally encephalomyelitis (transplacental transmission of the bacteria to embryo); in young domestic animals (sheep, cattle, pig, horse) enteritis, conjunctivitis, pneumonia, and polyarthritis. This disease has very high economic impacts.

Transmission mode: inhalation, less often by contact or alimentary.

Human disease: mammalian chlamydiosis is milder than psittacosis, with fever, headache, conjunctivitis, arthralgia, affections of urogenital tract; in severe cases pneumonia, renal failure, miscarriage but also women sterility as a consequence; rarely fatal. Risk procedures: assistance with lambing (especially women). 
Bio-containment: BSL-2.

Diagnosis and treatment: as for $C$. psittaci.

Geographical distribution: probably worldwide.

\section{Chlamydophila felis, C. caviae}

Source of infection: cat (C. felis), and guinea pig (C. caviae).

Animal disease: conjunctivitis, swollen eyelids, ocular and nasal discharge, pneumonia, occasionally chronic salpingitis.

Transmission mode: contact.

Human disease: conjunctivitis, in $C$. felis infection sometimes endocarditis and liver failure. A few zoonotic human infections have been reported.

Bio-containment: BSL-2.

Diagnosis and treatment: as for $C$. psittaci.

Geographical distribution: probably worldwide.

\subsubsection{Family Parachlamydiaceae [Order Chlamydiales]}

\section{*Parachlamydia acanthamoebae}

An obligate intracellular bacterium - endosymbiont of amoebae free-living in water.

Source of infection: river water, drinking water treatment plants, hospital water supplies.

Animal disease: abortions in ruminants.

Transmission mode: water-borne.

Human disease: pneumonia (purulent and interstitial), miscarriage, stillbirth and premature birth.

Bio-containment: BSL-2.

Diagnosis: PCR, isolation on cell cultures or by amoebal co-culture, serology (ELISA).

Treatment: specific treatment unknown, the bacterium is resistant to fluoroquinolones and other antibiotics.

Geographical distribution: probably worldwide.

\subsubsection{Family Simkaniaceae [Order Chlamydiales]}

\section{* Simkania genevensis}

An endosymbiont of free-living amoebae.

Source of infection: river water, drinking water treatment plants.

Animal disease: unknown.

Transmission mode: water-borne. 
Human disease: pneumonia.

Bio-containment: BSL-2.

Diagnosis: PCR, isolation on Vero cells or by amoebal co-culture, serology (ELISA).

Treatment: specific treatment unknown, the bacterium is resistant to fluoroquinolones and other antibiotics.

Geographical distribution: Israel, but probably worldwide.

\subsubsection{Family Waddliaceae [Order Chlamydiales]}

\section{Waddlia chondrophila}

Source of infection (natural host range): domestic ruminants.

Animal disease: abortions.

Transmission mode: aerogenic.

Human disease: miscarriage (described in 2007).

Bio-containment: BSL-2.

Geographical distribution: USA, Germany.

\subsubsection{Family Rickettsiaceae [Order Rickettsiales, Class Alphaproteobacteria]}

Rickettsial microorganisms of the families Rickettsiaceae and Anaplasmataceae are very small Gram-negative bacteria, coccobacilli to short rods about $0.6 \times 0.3 \mu \mathrm{m}$, intracellular parasites (in either nucleus - rickettsiae of spotted fever group, RSFG or cytoplasma of endothelial cells or leucocytes) and replicate by cross section. They possess most of enzymes the other bacteria have, form ATP (contrary to chlamydiae), exhibit a normal bacterial cell wall with muramic acid, and are stained with Giemsa, Giménez or Macchiavelli stains, poorly according to Gram. A natural reservoir of rickettsiae are arthropods of diverse systematic groups, in them they live usually as commensals, while in the organism of an "unnatural" host, e.g. man, they often cause an illness with fever, vasculitis (they attack selectively endothelial cells) and often also a rash or erythema (spotted fevers and typhus). According to the vector they can be divided into:

(a) louse-borne: Rickettsia prowazekii

(b) flea-borne: R. typhi, R. felis

(c) tick-borne: rickettsiae of the spotted-fever group (SFG): $R$. rickettsii, $R$. conorii, $R$. africae, $R$. sibirica, $R$. slovaca, $R$. japonica, $R$. australis; further Anaplasma phagocytophilum, Ehrlichia chaffeensis and other ehrlichiae

(d) mite-borne: $R$. akari, Orientia tsutsugamushi

(e) cercaria-borne (trematode larvae): Neorickettsia sennetsu. 


\section{(**) Rickettsia prowazekii}

Its genome is fully sequenced.

Source of infection (natural host range): man; infrequently the flying squirrel Glaucomys volans.

Animal disease: inapparent course.

Transmission mode: body louse (Pediculus humanus - TST demonstrated, TOT not) - rubbing infectious louse excreta into the skin; aerogenic (inhallation of dust containing louse excreta). About 15 cases of human infection from flying squirrel have been described in USA recently ( $R$. prowazekii was also isolated from the rodent) - it means that a limited sylvatic cycle of this rickettsiosis exists.

Human disease: epidemic typhus, with severe headaches, pains in limbs, high fever for 10-20 days, chills, rash on the trunk and limbs (4-7 days), meningeal signs, bronchopneumonia, haemorrhagies and a fatality rate in non-treated cases $10-50 \%$. Sometimes a recidiva even after 10-20 years, socalled Brill-Zinsser's disease, which is a re-activation of the agent persisting in lymphatic nodes or in adipose tissue. Big epidemics have been reported during wars, famin and natural disasters, especially in winter and early spring. Examples of the extensive outbreaks are "plague in Athens" 430 BC (most probably it was epidemic typhus, or combined with ergotism), 1490 Granada, 1528 Naples, 1542 Hungary, 1546 Beograd, 1618-1648 central Europe during 30-year War, 1640 England, 1914-1918 the Balkans during 1st WW (150,000 soldiers get infected solely in Austrian army), 19171922 Russia (30 million persons fell ill and 3 millions died), 1941-1945 Russia (about 20 million sick persons); 1997 sanatorium Lipeck in Russia (29 patients with Brill-Zinsser's disease); 1997-1998 Burundi and Rwanda: 50,000 to 100,000 patients in refugee camps, fatality rate up to $15 \% ; 1998$, an outbreak in Peru, and sporadic cases in Algeria, western Europe and USA (usually in homeless persons).

Bio-containment: BSL-3.

Diagnosis: symptoms; serology: CFT; AR (earlier also Weil-Felix AR with heterologous antigen used: Proteus OX-19), CFT, RIHA, ELISA, isolation (guinea pig), detection of rickettsiae in samples (IF, PCR).

Treatment: tetracycline, doxycycline, chloramphenicol.

Geographical distribution: Africa (Ethiopia, Algeria; Rwanda and Burundi, Zaire), Asia (e.g., Kazakhstan, 2000), South America, USA, Russia, western Europe.

\section{Rickettsia typhi (Syn. R. mooseri)}

Source of infection (natural host range): synanthropic rodents (brown rat, black rat), cat; dog; in the sylvatic cycle opossum (Didelphis marsupialis).

Animal disease: inapparent course. 
Transmission mode: fleas (TOT demonstrated) Xenopsylla cheopsis, Ctenocephalides felis and other species; aerogenic (fleas' excrements).

Human disease: murine typhus, with symptoms similar to epidemic typhus but milder - fever, heavy headaches, pains in limbs, dry cough for 2 weeks, usually a 6-day rash on chest and abdomen, elevated transaminases; fatality rate in untreated cases is $1-4 \%$; long convalescence (several months). For instance, 33 human cases of murine typhus were identified in USA in 2008.

Bio-containment: BSL-2/3.

Diagnosis: inoculation of blood samples in male guinea pig (scrotal reaction) or chick embryo; detection by PCR; serology (CFT, IFA, ELISA, in the past Weil-Felix with Proteus OX-19 and OX-2 antigen, latex agglutination), WB.

Treatment: doxycycline, tetracycline, chloramphenicol.

Geographical distribution: worldwide, but tropical and subtropical regions prevail; southern Europe, the Netherlands, Australia; especially harbours and coastal areas with markets (where rats are frequently present).

\section{Rickettsia rickettsii}

A prototype SFG rickettsia.

Source of infection (natural host range): rodents (Spermophilus, Marmota, Eutamias, Hydrochaeris), opossum, leporids, canids, occasionally birds.

Animal disease: inapparent course, but clinical disease in dogs.

Transmission mode (Fig. 8.18): ixodid ticks - in North America Dermacentor andersoni, D. variabilis (reservoir - TST and TOT demonstrated already

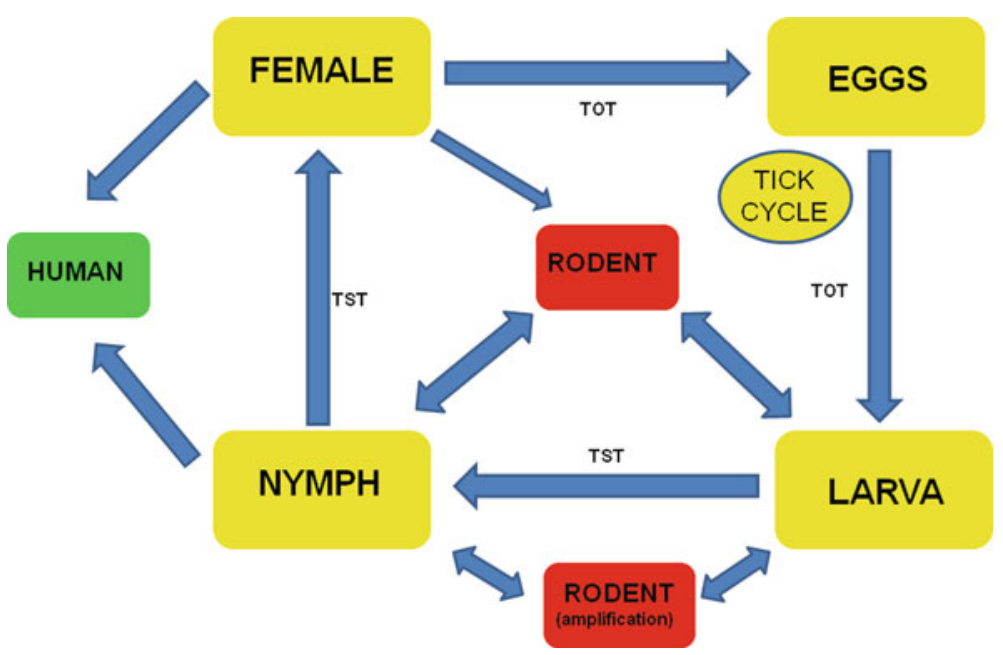

Fig. 8.18 Circulation of R. rickettsii in a natural focus of Rocky Mountain spotted fever (modified from Beaty and Marquardt 1996) (drawing by Ivo Rudolf) 
by H.T. Ricketts), also D. albipictus, D. parumapterus, infrequently Rhipicephalus sanguineus (Mexico, Brazil), Haemaphysalis leporispalustris (Costa Rica), Amblyomma cajennense and A. aureolatum (Brazil, Mexico); aerogenically (laboratory infections).

Human disease: Rocky Mountains spotted fever (RMSF), and Brazilian spotted fever - severe illness with symptoms similar to epidemic typhus, with a sudden and longterm fever, severe headache, myalgia, abdominal pains, vomiting, rash on ankles and wrists, bronchopneumonia, photophobia, CNS affection (meningism), haemorrhages of limbs and genitalia (gangrene of scrotum), hepatosplenomegaly, myocarditis, renal lesions (up to renal failure); thrombocytopenia, increased transaminases; fatality rate is about $20 \%$ in untreated sick persons (but in west Montana and in Brazil higher, $30-42 \%$ ), while in treated patients only $1-5 \%$. The annual incidence of RMSF in the USA increased five times from 0.14 to 0.70 per 100,000 population between 2000 and 2007.

Bio-containment: BSL-3.

Diagnosis: inoculation of blood samples in male guinea pig (scrotal reaction) or chick embryo; serology: in the past Weil-Felix AR with Proteus OX-19, OX-2; CFT, IFA, while at present microagglutination, IFA, PCR or immunohistochemistry of skin biopsies.

Treatment: doxycycline, tetracycline, chloramphenicol, ciprofloxacin.

Prevention: inactivated vaccine is slightly effective, it is not in use.

Geographical distribution: North America, Brazil (states Sao Paolo and Minas Gerais).

\section{Rickettsia slovaca}

A SFG rickettsia, genomically related to $R$. rickettsii. First described in D. marginatus ticks collected in Slovakia in 1968.

Source of infection (natural host range): rodents, cattle.

Animal disease: inapparent course.

Transmission mode: ixodid ticks Dermacentor marginatus, infrequently $D$. reticulatus and Rhipicephalus bursa.

Human disease: TiBoLa ("tick-borne lymphadenitis") or DeBoNEL ("Dermacentor-Borne-Necrosis-Erythema-Lymphadenopathy"), with an eschar (an infiltrate with a dark scar) in the place where the vector tick fed - often in the scalp, with alopecia and erythema around the eschar, fever, headache, often painful regional (usually cervical) lymphadenitis and maculo papular rash. Laboratory findings include thrombocytopenia and increased transaminase levels. The illness usually occurs in early spring (February to May) when the vector ticks start to be active. The first human case of TiBoLa was reported by T. Mittermayer et al. in eastern Slovakia in 1980.

Bio-containment: BSL-2. 
Diagnosis: symptoms, serology (ELISA, IFA), PCR.

Treatment: tetracycline, doxycycline.

Geographical distribution: Europe (Slovakia, Hungary, Poland, France, southern Germany, Austria, Switzerland, Slovenia, Croatia, Spain, Portugal, Italy, Greece, Bulgaria, Romania, Ukraine, Russia), Armenia, Africa.

\section{Rickettsia honei}

A SFG rickettsia, with a subtype called " $\boldsymbol{R}$. marmionii”.

Source of infection (natural host range): rodents (Rattus rattus).

Animal disease: inapparent course.

Transmission mode: ixodid ticks Haemaphysalis novaeguineae, Bothriocroton (Aponoma) hydrosauri and Ixodes granulatus.

Human disease: Flinders Island spotted fever. $R$. $m$. causes Australian spotted fever with headache, pharyngitis, cough, maculopapular rash, and arthralgia.

Bio-containment: BSL-2.

Diagnosis: symptoms, serology (ELISA, IFA), PCR.

Treatment: tetracycline, doxycycline.

Geographical distribution: Australia and Oceania, Thailand.

\section{Rickettsia conorii}

A SFG rickettsia with several subspecies: $R$. conorii conorii (its full genomic sequence is known), and closely related rickettsiae of Astrakhan fever ( $R$. conorii caspia), Israeli tick typhus ( $R$. conorii israelensis) and Indian tick typhus $(R$. conorii indica).

Source of infection (natural host range): dog and other canids; rodents, wild rabbit.

Animal disease: usually inapparent course, but fever, anorexia, lethargy, splenomegaly, and polyneuritis in dogs.

Transmission mode: ixodid ticks Rhipicephalus sanguineus (TOT), $R$. appendiculatus, R. pumilio (R. c. caspia), Haemaphysalis leachi (TOT), Hyalomma spp.

Human disease: Mediterranean spotted fever, boutonneuse fever (French: "fievre boutonneuse") was first described in 1910 - an infiltrate in the place where the vector tick fed with a dark scar (primary necrotic skin lesion "eschar", French "tache noir"), fever 1-2 weeks, headaches, arthralgia, myalgia, sometimes maculopapular rash, and regional lymphadenopathy in half of patients; increased transaminases AST and ALT; the complications might appear as pneumonia or intravenose coagulation; fatality rate is in untreated persons $1-5 \%$ but in places higher (e.g. in Portugal 32\% in 1997). The geographical forms of the disease are Israeli tick typhus and Indian tick typhus, and Astrakhan fever (maculopapular rash, petechiae and 
haemorrhagies due to thrombocytopenia) - the third geographical variant started to appear in south Russia in the 1970 s, until $1983>2,000$ cases were recorded (allegedly in connection with building of a petrochemical plant with a high level of $\mathrm{CO}_{2}$ emissions). In 1992, there was a large outbreak among American soldiers in Botswana.

Bio-containment: BSL-2.

Diagnosis: symptoms, serology (CFT, Weil-Felix Proteus OX-2 and OX-19 antigens, AR, IFA, WB), PCR, isolation on guinea pig.

Treatment: tetracycline, doxycycline, chloramphenicol.

Geographical distribution: the Mediterranean, Switzerland, the Balcans (Kosovo), the Black Sea region, North Africa, Uganda, Botswana, Asia (the Near East, India).

\section{Rickettsia sibirica}

SFG rickettsia related to $R$. conorii, and first detected in Siberia, 1939. $R$. mongolitimonae is newly classified as subspecies of $R$. sibirica ( $R$. sibirica mongolitimonae).

Source of infection (natural host range): rodents, leporids, hedgehogs, cattle. Animal disease: inapparent course.

Transmission mode: ixodid ticks (reservoir) Dermacentor (D. nuttallii, D. silvarum, D. marginatus), Haemaphysalis (H. concinna) and Hyalomma (H. asiaticum, $H$. truncatum).

Human disease: North-Asian (Siberian) tick typhus with fever, headache, regional lymphadenitis and papular rash (eschar).

Bio-containment: BSL-2/3.

Diagnosis: symptoms, serology (ELISA, IFA), PCR.

Treatment: tetracycline, doxycycline, minocycline.

Geographical distribution: northern Asia, Kazakhstan, Kirghizia, Japan, China, Korea, eastern Europe, Armenia, Pakistan; R.s. mongolitimonae was detected in Mongolia and China, but recently also as an autochthonous agent (several human cases) in Spain, Portugal, France, northern Africa, and Nigeria.

\section{Rickettsia africae}

A SFG rickettsia related to $R$. conorii and $R$. parkeri.

Source of infection (natural host range): canids, rodents, cattle; (equids?).

Animal disease: inapparent course.

Transmission mode: ixodid ticks Amblyomma hebraeum, A. variegatum (TST and TOT demonstrated) and Boophilus decoloratus.

Human disease: African tick bite fever (Kenyan, South-African, Nigerian) eschar (quite often multiple eschars, fever for about 1 week, headaches, arthralgia, myalgia, maculo-papular rash, regional lymphadenopathy; the course is usually milder than in the Mediterranean spotted fever). It occurs 
quite often in travellers to Africa (European hunters, safari participants, visitors of game parks) - the second most frequently identified cause for systemic febrile illness among travellers, following malaria.

Bio-containment: BSL-2.

Diagnosis: symptoms, serology (IFA, ELISA, WB), PCR from the skin biopsy (eschar), isolation in guinea pig.

Treatment: doxycycline (tetracycline, chloramphenicol).

Geographical distribution: sub-Saharan Africa, introduced in the Caribbean.

\section{Rickettsia parkeri}

A SFG rickettsia, closely related to $R$. africae, described in 1939 and previously thought to be non-pathogenic to man.

Source of infection (natural host range): rodents, domestic animals, birds.

Animal disease: inapparent course.

Transmission mode: ixodid ticks Amblyomma maculatum, A. triste, A. americanum, Dermacentor variabilis.

Human disease: American boutonneuse fever with eschar (area of necrosis) or multiple eschars at the site of tick bite, and rash. Other symptoms include mild headache, malaise, diffuse myalgia and arthralgia. The illness is emerging in southern and south-central states of USA since the beginning of twenty-first century.

Bio-containment: BSL-2.

Diagnosis: symptoms, serology (IFA, ELISA), PCR from the skin biopsy (eschar), isolation in guinea pig.

Treatment: doxycycline (tetracycline, chloramphenicol).

Geographical distribution: southern USA, South America (Uruguay, Brazil, Argentina).

\section{Rickettsia raoultii}

SFGR.

Source of infection (natural host range): probably mammals.

Animal disease: unknown.

Transmission mode: ixodid tick Dermacentor reticulatus, D. marginatus, and other Dermacentor spp.

Human disease: similar to TiBoLa - tick typhus, with an eschar, fever, headache, arthralgia, myalgia, maculopapular rash, regional lymphadenopathy.

Bio-containment: BSL-2.

Diagnosis: symptoms, serology (ELISA, IFA), PCR.

Treatment: tetracycline, doxycycline.

Geographical distribution: Africa - Morocco, Mali, Niger, Zimbabwe, Europe (Italy). 


\section{Rickettsia aeschlimannii}

SFGR.

Source of infection (natural host range): mammals (?).

Animal disease: unknown.

Transmission mode: ixodid ticks Hyalomma marginatum (H. m. marginatum, H. m. rufipes), Haemaphysalis punctata, Rhipicephalus appendiculatus, $R$. sanguineus, $R$. bursa, Ixodes ricinus.

Human disease: similar to the Mediterranean fever (tick typhus), with an eschar, fever, headaches, arthralgia, myalgia, maculopapular rash, and regional lymphadenopathy. Only four cases of $R$. aeschlimannii fever have been reported up to now; they were confirmed by IFA, WB, and cross-absorption studies.

Bio-containment: BSL-2.

Diagnosis: symptoms, serology (ELISA), PCR.

Treatment: doxycycline; resistant to rifampin.

Geographical distribution: southern Europe (Portugal, Spain, Croatia, Corsica, Sicily), Africa (Algeria, Morocco, Egypt, Mali, Niger, Zimbabwe, southern Africa), Kazakhstan.

\section{Rickettsia massiliae}

SFGR, first isolated in Marseille (France) from ticks in 1992.

Source of infection (natural host range): probably mammals.

Animal disease: unknown.

Transmission mode: ixodid ticks Rhipicephalus sanguineus, $R$. pusillus, $R$. turanicus (TOT in the latter species), $R$. muhsamae, $R$. lunulatus, $R$. sulcatus.

Human disease: several cases of a spotted fever-like illness with fever, eschar, and maculopapular rash due to $R$. massiliae have been reported in France and Spain since 2005.

Bio-containment: BSL-2.

Diagnosis: symptoms, serology (ELISA), PCR.

Treatment: tetracycline, doxycycline; resistant to rifampin.

Geographical distribution: France, Spain, Portugal, Italy (Sicily), Switzerland, Greece, Mali, Central Africa, USA.

\section{Rickettsia japonica, $R$. heilongjiangensis}

SFG rickettsiae, very similar to each other.

Source of infection (natural host range): rodents, hedgehog; dog, cattle (?).

Animal disease: inapparent course.

Transmission mode: ixodid ticks (reservoir) Haemaphysalis concinna and Dermacentor silvarum ( $R$. heilongjiangensis), H. japonica, H. longicornis, $H$. flava and Ixodes ovatus in R. japonica.

Human disease: Japanese (Oriental) spotted fever, increasingly occurring in Japan since 1984 . 
Bio-containment: BSL-2.

Treatment: tetracycline, doxycycline.

Geographical distribution: Japan, China, the Far East (Khabarovsk); R. heilongjiangensis occurs in northern Asia.

\section{Rickettsia helvetica, $R$. monacensis}

Closely related SFG rickettsiae ( $R$. monacensis was originally labeled as IRS3 strain).

Source of infection (natural host range): rodents, cattle.

Animal disease: mostly inapparent course, but pathogenic for Microtus and Apodemus spp.

Transmission mode: tick Ixodes ricinus, I. ovatus, I. persulcatus, I. monospinus, occasionally Dermacentor reticulatus and Haemaphysalis inermis (R. helvetica, Croatia and Hungary, respectively).

Human disease: fever with headache, myalgia, arthralgia, rarely chronic perimyocarditis or meningitis ( $R$. helvetica); rash not reported. $R$. helvetica was the cause of a subacute meningitis case in Sweden in 2006 (confirmed by nucleotide sequencing). With $R$. monacensis, two human cases were reported in Spain, 2006.

Bio-containment: BSL-2.

Treatment: tetracycline, doxycycline.

Geographical distribution: R. helvetica: Europe (Switzerland, France, Germany, Poland, Slovenia, Portugal, Italy, Austria, Hungary, Sweden, Denmark). $R$. monacensis: Spain, Italy, Germany, the Netherlands, Slovakia, Hungary, Albania, Bulgaria, Morocco, Tunisia, Japan.

\section{Other SFG Rickettsiae}

Presumptively associated with human illness are the tick-borne species and candidates:

Rickettsia canadensis (the vector is Haemaphysalis leporispalustris) in Canada; "R. amblyommii" (vectors A. americanum, A. cajennense, A. coelebs) in USA (Tennessee); "R. texiana", the probable agent of

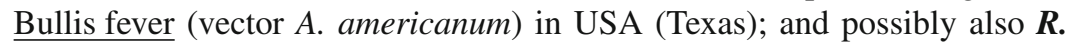
peacockii, $R$. rhipicephali, $R$. montana, and $R$. belli.

\section{Rickettsia australis}

This species is close to $R$. akari.

Source of infection (natural host range): rodents, marsupials (bush rats, bandicoots, opossum), canids.

Animal disease: inapparent course.

Transmission mode: ixodid ticks Ixodes holocyclus, I. tasmani, I. cornuatus. 
Human disease: Queensland tick typhus with fever up to 2 weeks, headache, eschar, regional lymphadenitis, and sometimes a generalized rash.

Bio-containment: BSL-2/3.

Diagnosis: isolation from the blood; serology (Weil-Felix AR, specific AR, CFT, RIHA).

Treatment: tetracycline.

Geographical distribution: Australia.

\section{Rickettsia akari}

Genomically similar to $R$. felis and $R$. australis.

Source of infection (natural host range): rodents - synanthropic (Mus, Rattus), less exoanthropic species.

Animal disease: lethal for mice.

Transmission mode: the mite Liponyssoides (Allodermanyssus) sanguineus (a mouse ectoparasite occasionally feeding also on man); laboratory infections.

Human disease: rickettsial-pox or vesicular rickettsiosis, with the course similar to African tick fever, but usually milder (however, sometimes lethal) - at first a primary ulcerating skin lesion (eschar) and regional lymphadenopathy, after a week follows fever with chills, headaches, myalgia, photophobia, and papulo-vesicular rash over the trunk and extremities (sometimes generalized) rash. Largely an urban zoonosis.

Bio-containment: BSL-2/3.

Diagnosis: symptoms, inoculaton of mice (the blood from febrile status), CFT, AR (Weil-Felix AR is negative).

Treatment: doxycycline, tetracycline (chloremphenicol).

Geographical distribution: towns in North America (New York: first described in 1946), Central America (Mexico), Eurasia (Russia, Ukraine, Croatia, southern Europe, Turkey, Korea) and South Africa.

\section{Rickettsia felis}

A SFG rickettsia originally labeled as "ELB agent", known since 1994, genomically closely related to $R$. akari.

Source of infection (natural host range): cat (dog, rodents), opossum (USA).

Animal disease: inapparent course.

Transmission mode: fleas Ctenocephalides felis (reservoir, TOT), Archaeopsylla erinacei (Germany), less often C. canis, Pulex irritans.

Human disease: flea-borne spotted fever - a febrile illness with rash, nausea, headache, joint pain, myalgia, and fatigue, occasionally skin rash, hepatitis; sometimes hospitalisation is necessary. In the years 1994-2006, 68 cases were reported, most frequently (40 cases) in Spain.

Bio-containment: BSL-2. 
Treatment: doxycycline, tetracycline (chloramphenicol).

Geographical distribution: Mexico, USA, Caribbean islands, Brazil, Argentina, Spain (also Canarian Islands), France, Germany (2 cases), Great Britain, Tunisia, Kenya, DR Congo, South Korea, Taiwan, Laos, Thailand.

\section{Orientia tsutsugamushi}

Source of infection (natural host range): exoanthropic rodents (wild rats), insectivores and other small mammals.

Animal disease: septicaemia in mice.

Transmission mode: larvae ("chiggers") of the mites, especially of the genus Leptotrombidium (reservoir - TST as well as TOT): L. deliense (in the whole geographical range; also a non-bacteraemic transmission by co-feeding has been described), L. akamushi (in Japan), L. pallidum (Japan, Korea, Russian Far East), L. scutellare (Japan, China, Malaysia), L. pavlovsky (the Far East), L. imphalum, L. chiangraiensis, L. fletcheri, L. aernicola, etc.

Human disease: scrub typhus, tsutsugamushi - sudden high fevers for up to 2 weeks, hyperhidrosis, severe headaches, lymphadenopathy, often eschar (e.g. in $90 \%$ of patients in China), macular rash on face, chest and abdomen, cough to pneumonia, gastrointestinal symptoms, splenomegaly, conjunctivitis, photophobia, CNS affection, often temporary deafness; average fatality rate $15 \%$ (range, $1-40 \%$, but up to $60 \%$ in untreated cases); Allied troops recorded a total of 20,000 tsutsugamushi cases during operations in 2ndWW, with a fatality rate of $25 \%$. In the 1970 s, scrub typhus was a frequent infectious disease among American soldiers also in the Vietnamese War. In South Korea, a total of 23,930 cases ( $68 \%$ serologically confirmed) were reported between 2001 and 2006.

Bio-containment: BSL-2/3.

Diagnosis: mouse inoculation (blood samples), serology (Weil-Felix AR, CFT, IFA, ELISA).

Treatment: tetracycline, doxycycline (chloramphenicol).

Prevention: effectivity of inactivated vaccine is low, it is not used.

Geographical distribution: southeastern, eastern and southern Asia (among others also the Himalayas in 2006), northern China, northern Australia, and Oceania, typically in grassy and bushy habitats that arose after cut down of tropical forests (Photo 5.32).

\subsubsection{Family Anaplasmataceae [Order Rickettsiales]}

\section{Ehrlichia chaffeensis, E. ewingii}

Genomic group E. canis s.l., also involves very similar E. muris and veterinary important Ehrlichia (Cowdria) ruminantium, the agent of heartwater disease of ruminants in Africa. 
Source of infection (natural host range): whitetail deer (a competent host, with ehrlichaemia, and reservoir as well), sika deer, dog (also E. ewingii), coyotte, cattle, goat; rodents (possibly only in east Asia, while not demonstrated as competent hosts in America).

Animal disease: chronic pancytopenia (thrombocytopenia) and a long-term ehrlichaemia in dogs infected with E. ewingii; E. canis, that is not transmissible to man, causes a severe disease in dogs while E. chaffeensis is only pathogenic to puppies.

Transmission mode: metastriate ixodid ticks Amblyomma americanum (E. chaffeensis - principal competent vector, E. ewingii), A. cajennense (E. chaffeensis), A. parvum (E. chaffeensis in Argentina), Dermacentor variabilis (E. chaffeensis in USA), Rhipicephalus sanguineus (E. chaffeensis in Cameroon), Haemaphysalis longicornis and H. flava (E. chaffeensis in Korea), H. yeni (E. chaffeensis in China).

Human disease: human monocytic ehrlichiosis (HME) - fever with chills, arthralgia, myalgia, headache, nausea, anorexia, diarrhoea, vomiting, aseptic meningitis (in about one-fifth of patients), rash similar to that in RMSF (30\% of cases - only with E. chaffeensis); thrombocytopenia, leucopenia, anaemia, increased level of AST; fatality rate 1-5\%. E. ewingii occurs more often in immunosuppressed individuals.

Bio-containment: BSL-2.

Diagnosis: blood smear (Giemsa: characteristic "morulae" of ehrlichiae in monocytes and macrophages); serology (IFA, ELISA, WB) with the antigen from E. canis; PCR with the blood samples, isolation on cell cultures (Vero, DH82 - canine histiocytes).

Treatment: doxycycline, tetracycline.

Geographical distribution: North America, Mexico, South America (Brazil, Chile, Venezuela, Argentina - E. chaffeensis), Japan, China, Korea, sporadically Europe (Portugal, Spain - E. chaffeensis), Africa (Cameroon, Mozambique, Mali, Burkina Faso).

\section{Anaplasma phagocytophilum s.l.}

The group of genomically very similar A. phagocytophilum, A. equi and HGE agent was earlier classified in the genus Ehrlichia as E. phagocytophila s.l. Another synonym is Cytoecetes phagocytophila. More distantly related species are A. platys and A. marginale. Within A. phagocytophilum s.l., there is a great variety of antigenic types (due to considerable variation of the outer membrane protein), genotypes, ecotypes and pathotypes.

Source of infection (natural host range): roe deer, red deer, fallow deer, sika deer, white-tailed deer (the deer are competent, amplifying hosts for variants such as Ap-1 that are probably not pathogenic for humans), moose, chamois; forest rodents like bank vole Myodes glareolus, yellownecked mouse Apodemus flavicollis, wood mouse A. sylvaticus, white-footed 
mouse Peromyscus leucopus (amplifying host and reservoir of the humanpathogenic variant Ap-ha), field vole Microtus agrestis, woodrat Neotoma fuscipes, squirrels, yellow-checked (redwood) chipmunk Tamias ochrogenys (a competent host).

Animal disease: tick-borne fever (TBF, first described in sheep and cattle in Scotland, 1932, although known in Norway for at least 200 years) - a severe febrile reaction accompanied with thrombocytopenia, leucopenia and anaemia of sheep, cattle, goat, dog, and horse. Infected hosts are more susceptible to other infections, e.g. to lameness caused by Staphylococcus aureus and called tick pyaemia. Persistent A.p. infections in lambs have been reported in Europe.

Transmission mode: ixodid ticks Ixodes ricinus, I. scapularis (TST, TOT), I. pacificus, I. spinipalpis, I. trianguliceps, I. persulcatus, I. ovatus.

Human disease: human granulocytic anaplasmosis/ehrlichiosis (HGA/HGE) with fever, headaches, myalgia, arthralgia, anorexia; bacteraemia, leucopenia, thrombocytopenia, increased levels of transaminases; fatality rate 5$10 \%$; convalescence long, usually 1 month (total weakness, fatigue). In addition, infected persons are more susceptible to other infections (due to immunosuppression). The disease was first described and elucidated in USA, 1994. Probably a majority of A. phagocytophilum strains are nonpathogenic for man (they are endosymbionts of ticks and their host is deer, e.g. the variant Ap-1), and only some strains belong to the pathogenic genotype (e.g., the variant Ap-ha). The situation is confusing especially in Europe, where often disproportionately high prevalence rates (detected by PCR) of A. phagocytophilum have been reported in ixodid ticks approaching those of Borrelia burgdorferi s.1., whereas incidence of HGA in Europe is substantially lower than for instance that of LB.

Bio-containment: BSL-2.

Diagnosis: blood smear (Giemsa stain: the bacteria form micro-colonies visible as morulae in neutrophil granulocytes), serology (IFA, ELISA with the antigen from A. equi); PCR, cultivation in human leukemic cells HL-60, vertebrate endothelial cells or in tick cell lines.

Treatment: doxycycline (or other oxytetracyclines) up to 3 weeks, children and pregnant women rifampicin.

Geographical distribution: North America, Europe (Great Britain, Ireland, Scandinavia, Switzerland, Italy, Slovenia, the Netherlands, France, Spain, Germany, Austria, Poland, Denmark, Slovakia, Czechland), Madeira, Siberia, China, Japan.

\section{Neoehrlichia mikurensis}

First isolated in Japan, 2004.

Source of infection (natural host range): rodents (Rattus norvegicus). Animal disease: unknown. 
Transmission mode: ixodid ticks Ixodes ovatus, I. ricinus.

Human disease: a case of septicaemia in Switzerland, 2009.

Bio-containment: BSL-2.

Diagnosis: blood smear, PCR.

Treatment: doxycycline.

Geographical distribution: Japan, China, Asian Russia, Slovakia, Switzerland, the Netherlands.

\section{Neorickettsia sennetsu (Syn. Ehrlichia sennetsu)}

Forms a genomic group with the nearly identical veterinary important $N$. risticii.

Source of infection (natural host range): parasites of freshwater fishes and molluscs - larvae (cercaria) of trematodes (for instance Nanophyetus salmincola in the USA).

Animal disease: Potomac horse fever - monocytic ehrlichiosis with fever and colitis (N. risticii).

Transmission mode: ingestion of cercariae that have as intermediate hosts freshwater molluscs and fish.

Human disease: sennetsu fever with lethargy, lymphadenopathy, haematological changes similar to those present in mononucleosis; mortality has not been recorded.

Bio-containment: BSL-2.

Diagnosis: serology (IFA, ELISA), inoculation in mouse (blood samples), blood smear (monocytes, macrophages), PCR. Cultivation impossible.

Treatment: tetracyclines.

Geographical distribution: Japan and SE. Asia; E.r. in USA and Europe.

\subsubsection{Family Bartonellaceae [Order Rhizobiales, Class Alphaproteobacteria]}

Six species have been documented to cause endocarditis in humans: Bartonella quintana, B. henselae, B. elizabethae, B. vinsonii subsp. berkhoffi, B. koehlerae, and B. alsatica.

\section{(**) Bartonella quintana}

Bartonellae were formerly classified as rickettsiae. However, they differ from them in many respects, e.g. by the ability of extracellular growth in vitro on (special) bacteriological media. B. quintana is genomically closely related to $B$. henselae, in fact its "descendant" that aroused by a reductive evolution of the genome. 
Source of infection (natural host range): man (reservoir); exceptionally cat and dog.

Transmission mode: excrements of body louse (Pediculus humanus); B. quintana replicates intensively in its intestinal epithelium extracellulary. In Africa (Gabon, 2005), DNA of B. quintana was detected in the flea Pulex irritans.

Human disease: trench fever (febris quintana) - chills, headaches, backaches and pains in limbs; 4-5 (or more) relapses at intervals of 4-6 days, sometimes even after years; endocarditis, bacillary angiomatosis (cutaneous and subcutaneous vascular lesions), rash (infrequently), long-term bacteraemia. During the 1 st WW, $>1$ million cases in the battlefields. Today the disease occurs occasionally among homeless people, alcoholics, drug abusers and AIDS patients.

Bio-containment: BSL-2.

Diagnosis: cultivation of blood samples in vitro (chocolate $\mathrm{BA}, 5 \% \mathrm{CO}_{2}, 35^{\circ} \mathrm{C}$ ), serology, xenodiagnostics (experimental infection of lice with the blood or urine from the patient).

Treatment: tetracycline, chloramphenicol.

Geographical distribution: Europe (Poland, Ukraine during the 1st WW, the Far East, north Africa, Mexico, the Andes (villages)); Seattle 1995, Moscow 1997-1998, Marseille 1998-2001, Utrecht 2001 (homeless people), central Africa (refugee camps).

\section{${ }^{* *}$ Bartonella bacilliformis}

Very small coccobacilli $0.3-1.5 \times 0.2-0.5 \mu \mathrm{m}$.

Source of infection (natural host range): man.

Transmission mode: sandflies (especially Lutzomyia verrucarum).

Human disease: bartonellosis, in two clinical phases: (1) Oroya fever (Carrión's disease - acute form - anaemia, with fatality rate 10- $40 \%$ when untreated); (2) verruga peruana (Peruvian wart - papular to verrucose secondary cutaneous form, with a low mortality) [Daniel Carrión autoinfected himself with the blood of a patient, and documented the two stages in 1885; he succumbed to the disease]. A total of about 7,000 workers died due to this disease during the building of the railway connecting Lima with Oroya in 1870.

Bio-containment: BSL-2.

Diagnosis: blood smear - Giemsa (intraerythrocytic localisation) in acute phase; skin biopsy in the secondary phase; haemocultivation (chocolate BA or agar with rabbit serum, 5-10\% $\mathrm{CO}_{2}$, the growth is slow); serology (CFT, AR).

Treatment: chloramphenicol, ampicillin, penicillin, streptomycin, tetracycline (in verruga peruana mainly against secondary microflora).

Geographical distribution: South America (valleys of the Cordillera mountains in Peru, Ecuador and Columbia). 


\section{Bartonella henselae, B. clarridgeiae, B. koehlerae, B. rochalimae, B. alsatica}

$B$. henselae is genomically closely related to B. quintana, in fact its "ancestor".

Source of infection (natural host range): cat (reservoir, 10-25\% of cats in USA and New Zealand are bacteraemic), rodents. In B. rochalimae, also red fox and gray fox (reservoir?), coyote, raccoon, rural domestic dogs. In B. alsatica, wild rabbit is a natural host.

Animal disease: usually inapparent course, but may cause reproductive failure in female cats, and endocarditis in dog (B. rochalimae).

Transmission mode: percutaneous by cats (scratching, biting); the cat flea Ctenocephalides felis (a competent vector, but flea-to-human transmission is obviously rare). [Often discussed possible transmission of $B$. henselae by ixodid ticks has not been proven, there are no clear supportive data].

Human disease: cat scratch disease (CSD) or felinosis, benign lymphoreticulosis, regional lymphadenopathy - especially in children, high fever with headaches, pharyngitis, exhaustion, sometimes conjunctivitis, nodular syndrome, arthralgia, a long-term rash (4-24 months), endocarditis, bacillary (epitheloid) angiomatosis (cutaneous, subcutaneous and other vascular lesions - especially in AIDS patients), persistent bacteraemia, infrequently chronic osteomyelitis, aseptic meningitis; no mortality. In the USA up to 20,000 cases annually, and from that number $>2,000$ need hospitalisation (mean incidence rate is $9 / 100,000$ population annually). In the Netherlands, the CSD incidence estimates are 2,000 per year, and 12/100,000 population. B. alsatica was first identified in 1999 in Alsace, France, as an agent of bacteraemia in healthy wild rabbits. In 2006, interest in B. alsatica increased when it caused a blood culture-negative endocarditis in a patient with lymphadenopathy who had contact with rabbits.

Bio-containment: BSL-2.

Diagnosis: clinical symptoms (primary cutaneous lesion - a papule, enlarged lymph nodes, etc.), anamnesis, biopsy of lymphatic glands, serology (IFA, CFT, ELISA - IgM: but there might occur cross reactions with $B$. quintana, Coxiella burnetii and Chlamydophila pneumoniae), intradermal test (Hanger-Rose); detection of antigen (IF, ELISA) or DNA (PCR) of the agent; the cultivation is quite difficult (BHI agar with $5 \%$ blood or chocolate BA, $5 \% \mathrm{CO}_{2}$ ) because the growth is slow $-10-40$ days $/ 35^{\circ} \mathrm{C}$.

Treatment: symptomatic; long-term antibiotic treatment (rifampicin, ciprofloxacin, azithromycin, gentamicin, or trimethoprim + sulphamethoxazole) is not always very effective.

Geographical distribution: B. henselae worldwide; B. clarridgeiae in USA, Chile, Israel, Japan, the Philippines, Indonesia, Thailand, France, Germany, the Netherlands and Spain; B. koehlerae in USA; B. rochalimae in Peru, Chile, USA and France; $B$. alsatica in France and Spain. 


\section{Bartonella elizabethae, B. vinsonii, B. grahamii}

Source of infection (natural host range): brown rat (reservoir for B. elizabethae) and other rodents (e.g., white-footed mice - reservoir, and groundhog in $B$. vinsonii, bank vole - reservoir, and yellow-necked mouse in B. grahamii); dog, coyote (B. vinsonii ssp. berkhoffii).

Animal disease: largely asymptomatic or subclinical course, but endocarditis in dogs infected with B. vinsonii.

Transmission mode: fleas and mites of rodents. For instance, main vector of B.g. is the flea Ctenophthalmus nobilis.

Human disease: endocarditis, neuroretinitis and other ocular manifestations (e.g. occlusion). B. elizabethae occurs in intravenous drug users and homeless people.

Bio-containment: BSL-2.

Diagnosis: serology (CFT), detection of antigen (IF, ELISA) or DNA (PCR) of the agent; the cultivation is quite difficult (BHI agar with $5 \%$ blood or chocolate BA, $5 \% \mathrm{CO}_{2}$ ), the growth is slow.

Treatment: symptomatic; the effect of antibiotics (doxycycline, rifampicin) is debatable.

Geographical distribution: the Americas (B. elizabethae, B. vinsonii), Sweden (B. elizabethae), Portugal (B. elizabethae), B. vinsonii also occurs in Europe. B. grahamii occurs in Eurasia (e.g., UK, Poland, Czechland, Sweden etc.), within the range of its reservoir host, Myodes glareolus.

\section{Bartonella schoenbuchensis, Candidatus Bartonella melophagi}

Source of infection (natural host range): deer (B. schoenbuchensis), sheep (B. melophagi).

Animal disease: asymptomatic course.

Transmission mode: in B. schoenbuchensis, deer keds (hippoboscids) Lipoptena cervi (Europe), L. mazamae (North America), while sheep keds Melophagus ovinus in $B$. melophagi.

Human disease: deer ked dermatitis (speculation at present, the aetiology has not yet been proved). $B$. melophagi was isolated from the blood of two women with skin lesion, myalgia, fatigue and dry cough (and pericarditis in one of them) in the USA. However, the clinical relevance of these findings remains to be established.

Bio-containment: BSL-2.

Diagnosis: serology (CFT), detection of antigen (IF, ELISA) or DNA (PCR) of the agent.

Treatment: symptomatic; the effect of antibiotics is debatable.

Geographical distribution: Germany and some other European countries, USA. 


\title{
8.3.8 Family Brucellaceae [Order Rhizobiales, Class Alphaproteobacteria]
}

\author{
Brucella abortus, B. melitensis, B. suis, B. canis
}

Non-motile Gram-negative coccobacilli or short small rods $0.6-1.5 \times 0.5-0.7 \mu \mathrm{m}$ without bipolar staining, not encapsulated. Several biotypes (biovars) in each species.

Source of infection (natural host range): domestic and wild ruminants - e.g., bison (B. abortus), elk (B. abortus), reindeer and caribou (B. suis biotype 4), domestic pig and wild boar (B. suis biotypes 1-3), brown hare (B. suis biotype 2), goat and sheep (B. melitensis), dog (B. canis).

Animal disease: Bang disease, brucellosis (B. abortus) - infectious abortions of cattle; arthritis; orchitis and epididymitis, sterility; chronic forms (often inapparent infections). Also other wild ruminants involved in the disease: bison, elk, reindeer. Porcine brucellosis (B. suis biovars 1, 2, or 3) causes reproductive failure in domestic and wild sows, orchitis in boars, arthritis or paralysis. B. suis biovar 2 is also the agent of the hare brucellosis - necrosis of spleen, liver and genital organs. Newly described species of brucellae cause illness in marine mammals (B. pinnipedialis, $\boldsymbol{B}$. ceti) or in terrestrial rodents (B. microti), but their pathogenicity for man is as yet unknown (although there are 3 reports on naturally acquired infection of humans with brucellae originating from marine mammals to date).

Transmission mode: alimentary (unpasteurized milk and cheese), percutaneous, aerogenic, per conjunctivae, or by contact; quite high tenacity in animal products, but not in external milieu. A number of laboratory infections have been described.

Human disease: brucellosis - a serious disease with acute or undulating fever (relapses are especially common with B. melitensis), hyperhidrosis, headaches, myalgia, arthralgia, lymphadenitis, hepatosplenomegaly, pericarditis, formation of granulomas, arthralgia, synovitis, myalgia, oedema of legs (B. melitensis), osteomyelitis, damage of kidneys and some other organs (orchitis, endocarditis); fatality rate in untreated cases is low, 2-5\%. Relatively frequent are chronic forms leading to permanent disability. Occupational disease: veterinarians, animal keepers and attendants, meat industry workers, hunters, medical and veterinary microbiologists are at risk.

Bio-containment: BSL-2/3.

Diagnosis: cultivation of the blood and tissue samples (liver agar, glucose agar with serum, $5 \% \mathrm{CO}_{2}$ ), inoculation of guinea pig (increased laboratory risk), PCR, serology (AR - cross reactions with Francisella tularensis and Yersinia enterocolitica serovar $\mathrm{O} 9$, sometimes it occurs so-called prozonal effect - the serum sample does not react with the brucella antigen at low dilutions but starts to react at higher dilutions, Rose Bengal AR; CFT - specific, Coombs test for incomplete antibodies, etc.), intradermal test. Some species of the 
genus Brucella can be misidentified as Ochrobactrum (e.g., O. intermedium) when using commercial biochemical test kits because their reactions are similar.

Treatment: difficult - doxycycline (tetracycline) + streptomycin; rifampicin + doxycycline combination for $\geq 6$ weeks; cotrimoxazole. No steroid administration.

Prevention: eradication programs in domestic animals (cattle), milk pasteurization.

Geographical distribution: nearly worldwide (in Europe especially the Mediterranean), in some places Bang disease was eradicated (e.g. in Czechland).

\subsubsection{Family Francisellaceae [Order Thiotrichales, Class Gammaproteobacteria]}

\section{Francisella tularensis}

Non-motile very small spherical to ovoid $(0.3-1.0 \times 0.2-0.5 \mu \mathrm{m})$ Gram-negative and facultatively intracellular bacteria. The species consists of several subspecies: F. tularensis tularensis (type A - "nearctica", the most virulent one for mammals), F. t. holarctica (type B), F. t. mediasiatica, and the low virulent F. t. novicida (only 5 patients with suspected infection with this subspecies have been reported). Another species of the genus Francisella is F. philomiragia, living saprophytically in water, being of low pathogenicity for man. There are also ichthyopathogenic francisellae recorded in marine fish farms in USA, Japan and Norway which are not pathogenic for endothermic vertebrates and are unable to grow at $37^{\circ} \mathrm{C}$. Very interesting are the francisellae living as endosymbionts in metastriate ixodid ticks especially of the genus Dermacentor in the Holarctis. The new findings indicate two probable trajectories of evolutionary adaptation from saprophytism to parasitism within the genus Francisella: (a) via water organisms; (b) via ixodid ticks.

Source of infection (natural host range): leporids, rodents (Microtus, Arvicola, Ondatra, in Canada also Castor - especially during overpopulation of the rodents and tularaemia epizootic in process within them); but never a tularaemic patient. Very recent data show that $F$. tularensis could also be an endosymbiont or commensal of water amoebae of the genus Acanthamoeba, the bacterium being able to replicate in these protozoa and survive in their cysts for several weeks. The interaction between aquatic and terrestrial cycles of $F$. tularensis is largely unknown.

Animal disease: tularaemia (usually lethal epizootics of hares, rabbits and rodents; sometimes sheep - only with the type A; subcutaneous nodules in dogs).

Transmission mode (Fig. 8.19): unusually varied - direct contact (e.g. skinning the infected animals); bites of ixodid ticks Dermacentor (reservoir, 
Fig. 8.19 Natural cycles of Francisella tularensis (drawing by Ivo Rudolf)

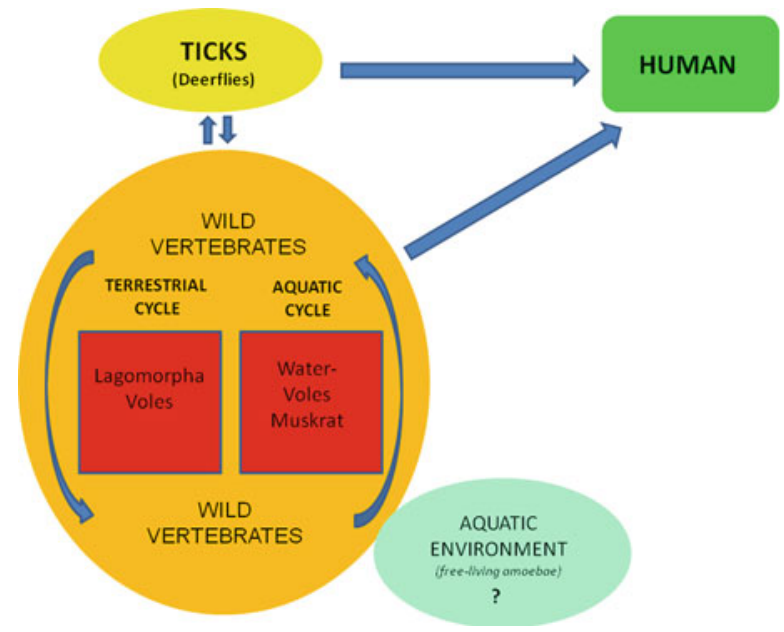

TST and TOT demonstrated) - D. variabilis, D. andersoni, D. reticulatus, Amblyomma americanum, rarely Ixodes ricinus complex, and in certain areas also mechanical transmission by bites of tabanids Chrysops spp. ("deerfly disease" - USA, Russia) while reports on transmission by mosquito bites are unsubstantiated at present (Scandinavia); alimentary (water or food contaminated by infected rodents); aerogenic (inhalation of contaminated aerosol or dust during the work with hay, straw or other agricultural products including sugar beet - outbreaks in sugar refineries in central and eastern Europe: Austria, Czechland, Slovakia, Ukraine). An unusual air-borne outbreak of tularaemia occurred among hunters (39 cases, 1 person died) in Hesse, Germany, 2005: the infection occurred when aerosol was generated during rinsing with a water hose of disemboweled hares (some of them being infected) after the hunt. High tenacity of the microbe at low temperatures at $4^{\circ} \mathrm{C} \mathrm{F}$. tularensis survives up to 4 months. Interestingly, F. tularensis survives in Acanthamoeba castellanii cysts for up to several weeks; trophozoites of this amoeba encyst rapidly in response to infection with $F$. tularensis.

Human disease: tularaemia - sudden fever, chills, headache, backache, hyperhidrosis, diarrhoea, anorexia; then according to the entrance gate different clinical forms: (1) (ulcero-/oculo-)glandular (bubonic) with the primary ulcer on the skin and regional painful lymphadenitis; (2) pulmonary (pneumonia); (3) abdominal (typhoid, oropharyngeal, gastrointestinal); or (4) generalized (septic); fatality rate is about $5 \%$ (but up to $30 \%$ in the typhoid form) in USA; while in Eurasia $<3 \%$ (due to absence of the highly virulent type A tularaemia); convalescence is long (2-3 months or more), especially in improperly treated illness. Occupational disease: farmers, hunters or trappers of leporids and big rodents (caught for skin, e.g. muskrats), workers in veterinary laboratories. There was a big outbreak of hare/rodent-borne tularaemia in Central Europe (Austria, Czechoslovakia) in 1936/1937, with 
many hundreds of patients. A recent (1999-2000) outbreak of largely alimentary tularaemia in Kosovo involved 327 confirmed cases; the main source were rodents, overpopulated during the Civil War in this region. The first two outbreaks of tularaemia in Spain occurred in 1997 and 1998, and were associated with hare hunting (585 patients) and crayfish (Procambarus clarkii) fishing in a contaminated freshwater stream, respectively. In the USA from 1990 to 2000, the average annual number of human cases of tularaemia was 124 (the range, 86-193); however, prior to 1950 there were many hundreds of cases annually (maximum in 1939: 2,291 reported patients). For comparison, in the Central-European Czechland (about 10 million population), the annual incidence averaged in the same decade 75 cases (the range, 12-225); an absolute maximum of tularaemia incidence in Czechland was recorded in 1962, with a total of 1,468 reported (serologically confirmed) patients.

Diagnosis: symptomatology, anamnesis, serology (AR, ELISA etc. - possible cross-reactivity with brucellae), subcutaneous inoculation of adult laboratory mouse (MLD is 1 cell!), cultivation of the blood or ulcer secreta samples on TGKA (glucose BA with thioglycollate) or cysteine BA, PCR; past infection could well be detected by an intradermal test with tularin; IF microscopy of lesion secreta, immunohistochemistry.

Bio-containment: BSL-2/3. (MID for man is as low as 10 viable cells applied intradermally or by inhalation). Considered as a bioterrorist agent (but only the type A tularaemia might be dangerous).

Treatment: streptomycin, gentamicin (tetracycline, chloramphenicol); exstirpation of enlarged lymph nodes.

Prevention: vaccination by scarification (the attentuated Russian strain, LVS) but routinely the vaccine is inaccessible.

Geographical distribution: holarctic (Eurasia and North America). F. t. tularensis (A) only occurs in North America, and F. t. mediasiatica only in Central Asia. For a natural focus of tularaemia, see Photo 5.42.

\subsubsection{Family Legionellaceae [Order Legionellales, Class Gammaproteobacteria]}

\section{${ }^{*}$ Legionella pneumophila (L. micdadei, L. bozemanii, L. longbeachae, L. anisa etc.)}

Poorly stainable Gram-negative aerobic rods $2-6(15) \times 0.3-0.9 \mu \mathrm{m}$, motile and facultatively intracellular. Up to now about 15 species (with a number of serotypes) of legionellae pathogenic to man have been described, the most important being the serotypes 1 and 6 of L. pneumoniae causing 80-90\% of cases. The group of so-called "legionella-like amoebal pathogens" (LLAP) is formed by parasites of amoebae; interestingly, antibodies to LLAP were detected in up to $20 \%$ of patients hospitalized with pneumonia of unclear aetiology in USA and Canada in 1995. 
Source of infection: water (for industrial purposes, heated water; water in air-conditioning systems, cooling towers in industrial plants, gas turbines, buildings' water systems, shower heads, whirlpools, warmwater mains, water sprayers, rainwater in puddles on asphalt roads during warm weather); a saprophytic and thermophilic microbe (the growth optimum is about $40^{\circ} \mathrm{C}$ ), often associated with other thermophilic bacteria and protozoa, e.g. cyanobacteria, Flavobacterium, amoebae (legionellae are endosymbionts of Acanthamoeba, capable to replicate in them). They often form biofilms in water habitats (water mains, cooling towers, etc.) and are resistant to standard disinfection compounds and measures. Potting soil is an alternative infections source of L. pneumophila and other Legionella species (especially in Australia and New Zealand, but rarely in Europe or North America).

Transmission mode: aerogenic - inhalation of aerosol (water droplets) containing the bacteria.

Human disease: two clinical forms - (1) legionellosis (Legionnaires' disease, LD) - pneumonia with high fever (up to $41^{\circ} \mathrm{C}$ ), chills and dry to spasmodic cough, headache, neurological and psychotic disturbances (confusion, anxiety), somnolence, vomiting, diarrhoea, damage to visceral organs (liver, kidneys); fatality rate $10-25 \%$; the disease is most dangerous for smokers and immunosuppressed persons; (2) Pontiac fever (the agent is, e.g., L. anisa), a flu-like illness with a sudden onset, myalgia and headache, but without any pulmonary affliction; mortality has not been reported. Very susceptible to both forms of legionellosis are immunosuppressed persons. Epidemics of legionellosis: first known in 1976 during a meeting of American legionnaires in Philadelphia: 221 patients (34 died; the agent, L. pneumophila was isolated and identified from the air-conditioning system in the meeting hotel); retrospectively (serologically), another outbreak of atypical pneumonia with 19 cases including 14 fatal in a psychiatric clinic in Washington was resolved as legionellosis; 1977, five smaller epidemics of legionellosis (total about 135 cases) in Ohio, Vermont, Tennessee, Los Angeles (USA) and Nothingham (UK); 1985, an outbreak (14 cases, 3 fatal) of LD after a banquet in Michigan; 1989, 34 LD cases (2 fatal) in Louisiana; 1998, 45 cases of Pontiac fever in Wisconsin (caused by a whirlpool); 1998, Institute of Clinical and Experimental Medicine in Prague 13 patients with transplanted organs ( 8 died; the source of LD was contaminated warm water in the institution); 1999, Bovenkarspel (the Netherlands) 233 LD cases (22 died) after a visit of exhibition of consumer goods including warmwater whirling baths (the source of infection); 1999, 22 cases of Pontiac fever in a hotel in Georgia, USA; 2000, 20 cases of Pontiac fever (from a hotel whirlpool) in Wisconsin; 2000, Melbourne 101 LD patients (5 died) after a visit of sea aquarium; 2000, Vigo (Spain) 28 LD patients were infected during hospitalisation due to other disease, and 3 died; 2002, 117 cases of Pontiac fever caused by L. anisa at a restaurant in Tennessee; 2004, 66 cases of Pontiac fever among visitors of a hotel in Oklahoma; 2010, Ulm 
(Germany) 65 patients (5 deaths; a community outbreak of LD associated with a water cooling system), and Barcelona (43 cases of Pontiac fever).

Bio-containment: BSL-2.

Diagnosis: serology (paired serum samples: IFA, AR, ELISA); detection of the agent in sputum and other excreta or by bronchoalveolar lavage (IF; PCR could detect viable, but non-cultivable cells); cultivation on a special BCYE agar medium containing yeast extract, cysteine, iron ionts and charcoal in 5\% $\mathrm{CO}_{2}$ (LLAP need the presence of amoebae for growth); antigen detection in urine (ELISA, PCR).

Treatment: clarithromycin, erythromycin, tetracycline, levofloxacin, azithromycin, rifampicin (penicillin and ampicillin are ineffective).

Geographical distribution: worldwide, but (surprisingly) mainly in the temperate zone. L.l. occurs in Australia and USA while in Europe sporadically.

\subsubsection{Family Coxiellaceae [Order Legionellales]}

\section{Coxiella burnetii}

Pleomorphic minute coccobacilli or short rods $0.4-1.0 \times 0.2-0.4 \mu \mathrm{m}$. Obligate intracellular parasite with a greatly reduced genome, able to multiply also in phagolysosomes of vertebrates. It reveals a phase variation: virulent phase I (S), and avirulent II (R). During a passage in the chick embryo, the coxiellae undergo the transformation I $\rightarrow$ II (which corresponds to a deletion mutation expressed by a surface lipopolysaccharide change), but the reverse transformation II $\rightarrow$ I is possible by passaging through a mammal or a tick. Only the phase I occurs in nature. The strains of $C$. burnetii obviously differ substantially in their virulence. (In the past, the genus Coxiella was assigned to rickettsiae.)

Source of infection (natural host range): all domestic ruminants (cattle, sheep, goat), less rodents, insectivores, and other wild mammals (including ruminants, and marsupials - kangaroos in Australia), occasionally pigeons.

Animal disease: coxiellosis - inapparent persistent infection; sometimes abortions or pneumonia.

Transmission mode (Fig. 8.20): aerogenic - by aerosol (foetal fluid and placenta of infected cows, ewes, and goats contain up to 109 coxiellae per gram - the infection exacerbates before delivery) and dust (straw, hay; dispersal by wind), often also alimentary (raw milk of goat and sheep: C. burnetii may resist even pasteurization $30 \mathrm{~min} / 60^{\circ} \mathrm{C}$ ), percutaneous (by bites of metastriate ticks, e.g. Rhipicephalus sanguineus, Dermacentor marginatus, Hyalomma lusitaniae - more often in subtropical regions; tick excrements contain up to 1010 coxiellae per gram; TOT in the ticks was demonstrated). C. burnetii has a very high tenacity in milieu: it resists drying and light exposure for many months: for instance on wool, cotton or in tick excrements it remains viable for a number of months, and in the soil for up to 


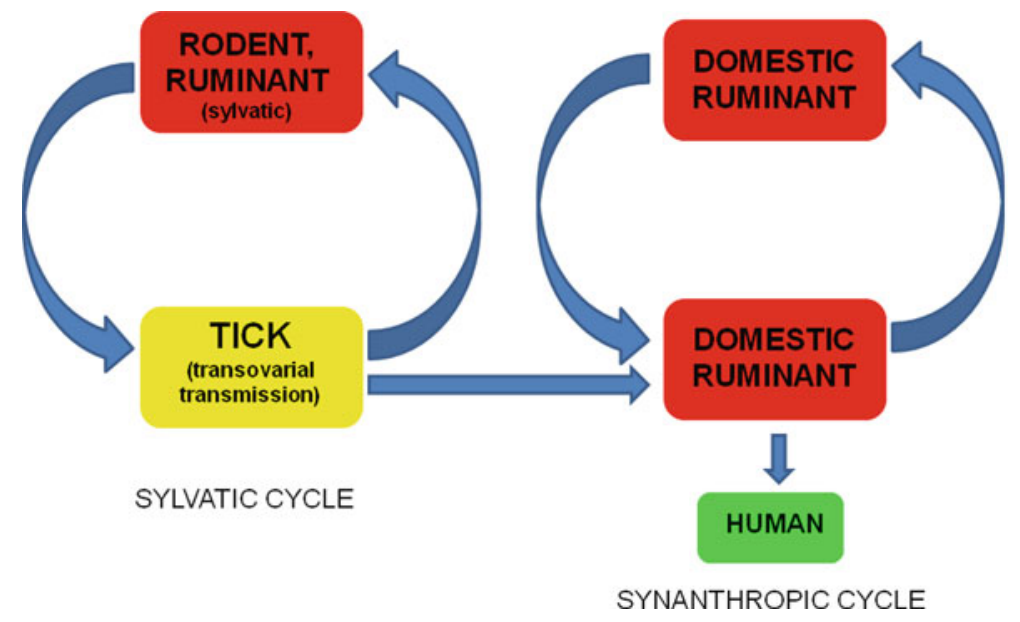

Fig. 8.20 The cycles of Coxiella burnetii (drawing by Ivo Rudolf)

20 days. The agent is extremely contagious and man is highly susceptible to infection. Exoanthropic cycle: rodents and wild ruminants (deer) + ixodid ticks (Metastriata). Synanthropic cycle: among domestic ruminants (contact). Some flies feeding on the faeces, milk, carcasses, or blood of infected domestic animals can spread $C$. burnetii mechanically.

Human disease: Q fever (Q meaning “query”), first described (by Edward Derrick) in butchers working in a slaughterhouse in Brisbane (Queensland, Australia) in 1935-1937 - sudden fever and chills, often recurrent, hyperhidrosis, weakness, intense headaches, fatigue, myalgia, arthralgia, atypical pneumonia (infiltrate, but not always), weight loss, myo- and endocarditis, rash does not occur (contrary to rickettsioses), sometimes meningitis; hepatitis (increased levels of transaminases); fatality rate $\leq 1 \%$; often inapparent or abortive infections (one-third to one-half of cases), on the other hand also chronical course occurs in 1-3\% of cases, accompanied with a latency period of several years (the agent persists in liver and spleen) and a characteristic subsequent endocarditis, sometimes granulomatous hepatitis (granulomas could also appear in the bone marrow). Reactivation of a latent or chronic infection can occur in gravidity (coxiellae are then present in placenta). Many smaller epidemics occur often in some Balkan countries, e.g. in Bulgaria have been reported hundreds of cases in conjuction with a rapid expansion of goat breeding since the 1990s. A very similar story is the recent (2008-2010, still ongoing) major outbreak of Q fever in the Netherlands where many farmers moved from cattle and pig breeding to goat (and sheep) breeding in recent years (there were $>350,000$ goats present in the Netherlands in 1999); 86 commercial dairy goat farms (i.e. $24 \%$ of all commercial goat farms) have been involved as of April 22, 2010, and the incidence of $\mathrm{Q}$ fever in humans has been unusually high. This epidemic started 
sneakingly, with only 5 human cases in 2005, 12 cases in 2006, 127 cases in 2007 , but as many as 1,014 cases were reported in 2008 (with one fatal case), 2,357 cases ( 6 fatalities) in 2009, and 421 cases in 2010 (as of end July); the situation resulted in a mass culling of goats ( $>10,000$ animals). Recently, new cases of Q fever also occur in Belgium, Germany (848 human cases were reported in 2006-2009), France, UK, Canada, USA, and Australia. In 2009, several patients with Q fever endocarditis were described in a settlement in eastern Greenland - the likely animal source included sled dogs and seals. $\mathrm{Q}$ fever is an occupational disease with a risk for breeders and attendants of small domestic ruminants (goat, sheep), veterinarians (delivery in ruminants), butchers; also many laboratory infections are known. For instance, a teacher and 33 students (of 48 exposed) acquired Q fever during veterinary practice in a Slovenian sheep farm in 2007.

Bio-containment: BSL-3.

Diagnosis: serology (micro-AR, IFA, CFT; antigen phase II indicates acute infection, while antigen I confirms the chronic stage of Q fever; in ELISA there can occur cross-reactions with Chlamydophila psittaci), intraperitoneal inoculation of the blood sample in guinea pig, mouse or on chick embryo (patients have intense bacteraemia during acute phase of the illness, but the isolation is a risky operation); staining after Giménez or Giemsa, immunohistochemistry; PCR.

Treatment: doxycycline, tetracycline, chloramphenicol (they shorten febrile period, but do not kill the intracellularly localized coxiellae), cotrimoxazole in children and pregnant women; a recommended combinations in chronic cases of $\mathrm{Q}$ fever are doxycycline with hydroxychloroquine, rifampicin or cotrimoxazole.

Prevention: Australian vaccine is approved for humans (it is prepared from the phase I); the protection is assumed to last 10 years. Culling of infected small ruminant herds according to the "test (bulk milk samples tested by PCR) and slaughter" policy. In addition, mandatory vaccination of small ruminants started in the Netherlands in 2009.

Geographical distribution: worldwide, most often in steppe habitats with brush of the Mediterranean and Black Sea regions (Photo 5.31), or in analogous Australian habitats.

\subsubsection{Family Enterobacteriaceae [Order Enterobacteriales, Class Gammaproteobacteria]}

\section{Salmonella enterica}

Motile facultatively anaerobic Gram-negative rods not fermenting lactose and producing hydrogen sulphide; the only for man pathogenic species of the genus, with 6 subspecies; virtually all serovars pathogenic for man are in the subspecies $S$. e. enter$i c a$, including also anthroponotic $S$. Typhi and $S$. Paratyphi. The serovars (today a 
total of $c .2560$ but those pathogenic for man are only about 100 from the groups B, C, D and E) are differentiated according to the Kauffmann-White scheme based on thermostable somatic antigens $\mathrm{O}$ (polysaccharides of cell wall) and thermolabile antigens H (from German "Hauch") of two phases (flagellar proteins); sometimes is also present thermolabile capsular Vi-antigen, masking the $\mathrm{O}$ antigen.

Source of infection (natural host range): fowl (especially $S$. Enteritidis: it contaminates the eggs in the ovary and sometimes even penetrates eggshell), rodents ( $S$. Typhimurium), pig ( $S$. Derby), cattle ( $S$. Typhimurium, $S$. Dublin, $S$. Enteritidis), sheep ( $S$. Montevideo), domestic mouse, synanthropic birds (gulls, house sparrow, collared dove, feral pigeon: $S$. Typhimurium, $S$. Enteritidis and other serovars), some ectothermic vertebrates.

Animal disease: usually inapparent course, or enteritis acute (young animals) or chronic (adults); abortions. Salmonellae are able to persist for a long time in visceral organs of mammals (spleen, liver, kidney, biliary duct) and in oviducts of fowl. $S$. Dublin and $S$. Pullorum (both not transmissible to man) cause severe disease in cattle and in chickens, respectively.

Transmission mode: alimentary - typical "food-borne disease" (mainly via contaminated eggs with $S$. Enteritidis and meat; MID for man is quite high, usually 106 , exceptionally $102-103$, but in some highly virulent strains allegedly 101 cells will do; some salmonellae easily replicate in foods at room temperature); direct contact (nosocomial infections). Great tenacity of $S$. Enteritidis (and other zoonotic salmonellae): e.g., in chicken droppings it survives viable $\geq 1$ year.

Human disease: salmonellosis, one of the most frequent and important zoonoses - gastroenteritis with a very short incubation period (6-48 h), watery diarrhoea (about 10 days), headache, abdominal pain, nausea, vomiting, fever, usually associated with haematogenic and lymphogenic dissemination of the agent, sometimes resulting in meningitis (especially in children); fatality rate is low, about $0.1 \%$ (mortality only occurs in toddlers and old patients - via critical dehydratation). The most frequent serovars are $S$. Enteritidis (frequent phagotypes are European PT4 and American PT8; - they have been panzootic and pandemic in the 1990s) and $S$. Typhimurium, less common are e.g. S. Virchow, S. Agona, and a number

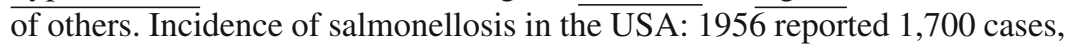
while 1965 already 20,865. A similar increase of incidence was recorded in European countries; for instance in Czechland: 1,981 reported cases in 1955, 4,975 in $1965,7,678$ in $1975,8,492$ in 1985 , and 52,588 in 1995. A considerable increase of the incidence in Czechland was reported in 1989, from 11 to 24 thousands of cases (it was caused by import of pandemic strain of the serovar $S$. Enteritidis from west Europe and USA), and the subsequent mean salmonella morbidity in the years 1990-2000 was 43,450 cases annually. While the incidence rate of salmonellosis per 100,000 population in Czechland was 385 in 1997, the average for Europe in the same year was 20130 , but it could be biased by underreporting (or no obligatory reporting) of 
the disease in some European countries. An instructive outbreak of salmonellosis occurred in Minnesota in 1994 with 224,000 patients (the largest salmonellosis outbreak in the U.S.A.): infective vehiculum was ice cream, and the mechanism was contamination with $S$. Enteritidis of a tank transporting egg melange used for preparation of that ice cream. The economical costs of salmonellosis can be high: it has been calculated that diagnosis and therapy of one human case of this disease in EU countries will cost on average 1,100€. [Remark: typhoid fever (typhus abdominalis) and paratyphoid fever, caused by $S$. Typhi and $S$. Paratyphi, respectively, are anthroponoses characteristic by a subsequent chronic asymptomatic carriership in 1-10\% of patients].

Bio-containment: BSL-2.

Diagnosis: cultivation of faecal samples in semiselective enrichment fluid media, e.g. tetrathionate or selenite enrichment broth, with a subsequent inoculation on selective solid agar media with deoxycholate, brilliant green etc. (MAL, XLD, SS, Rambach, Wilson-Blair and others); serotypization and phagotypization (PT), PCR (RFLP).

Treatment: rehydration (antibiotics prolong the carriership stage; they are used only in necessary cases and after sensitivity testing, especially in toddlers); Endiaron N.

Geographical distribution: worldwide.

\section{Escherichia coli - Enteropathogenic and Enterohaemorrhagic Strains}

Straight rods $1-6 \times 0.3-1.0 \mu \mathrm{m}$ with flagella (peritricha), fermenting lactose, with a number of serovars (160 antigenic types O, 56 types $\mathrm{H}, 80$ types K/Vi). The strains pathogenic for man are labeled as E. coli enteropathogenic (EPEC), enterohaemorrhagic (EHEC), enterotoxigenic (ETEC), enteroinvasive (EIEC) or enteroaggregative (EAEC); usually formed toxins include verotoxin (VTEC: e.g. in O157) or thermostable "shiga-like toxin" Stx 1 and Stx 2 (STEC); the most frequent antigenic types of these bacteria are O157:H7, further O111 and O26.

Source of infection (natural host range): cattle (reservoir, about $1 \%$ of the animals has EHEC in faeces); less often sheep, goat, pig, domestic dog and cat (pet contact), game animals (deer); VTEC isolates O157 also found in fowl. Seagulls can be competent hosts of multi-drug resistant $E$. coli. In addition, asymptomatic human carriers of some of these pathogenic strains have been revealed.

Animal disease: inapparent course or colibacillosis - diarrhoea in young domestic mammals (calf, lamb, piglet) and fowl.

Transmission mode: alimentary - contaminated hamburgers and other meat, contaminated vegetables (lettuce, spinach, salads from biofarms, vegetable sprouts) and fruit, contaminated water or milk; MID very low, only 70 cells during an epidemic in USA 1992/1993; by contact. EHEC strains tolerate 
even very acid conditions ( $\mathrm{pH} 2)$, temperature of $75^{\circ} \mathrm{C}$, and have marked tenacity in excreta (they survive $>100$ days). Infectious disease in man by EHEC strains from domestic animals was firstly reliably documented as a zoonosis in 1967. Mechanical transmission of E. coli O:157 by flies was also observed.

Human disease: colibacillosis - severe bloody diarrhoea (haemorrhagic colitis in VTEC), in 5-15\% of patients (STEC, VTEC), the infection sometimes progresses to renal failure (haemolytic- uraemic syndrome, HUS: most often with strains $\mathrm{O} 157, \mathrm{O} 26, \mathrm{O} 111, \mathrm{O} 103$ and $\mathrm{O} 145$, causing anaemia, thrombocytopenia, renal insufficiency to anuria brain oedema, and fatality rate of 3-5\%). In USA, EHEC has been observed since 1982, but the first patient was described already in 1975; an epidemic with >500 cases ( 4 children died) occurred in 1992/1993. In the years 1982-1998, 4,384 cases were recorded in USA. Annual incidence rate of EHEC in USA approximates 1-6 per 100,000 population (the average is 2 , and 250 patients die); in Czechland is the mean 12 per 100,000, and about 100 patients are infected with $\mathrm{O} 157$ annually. The number of VTEC cases in the European Union was 2,905 in 2007. A major outbreak of EHEC occurred in Toronto area in Canada in 2000 when $>2000$ people got infection (and 6 children died) - the infection vehiculum was a contaminated water from a farm.

Bio-containment: BSL-2.

Diagnosis: cultivation of faecal samples (differentiating MacConkey agar with sorbitol, so-called SMAC, or even more selective Rainbow O157 agar), with a subsequent typization and toxigenicity testing.

Treatment: rehydration, exceptionally antibiotics (ampicillin, cephalosporins). Geographical distribution: worldwide.

\section{Yersinia enterocolitica}

Slowly motile (but non-motile at $37^{\circ} \mathrm{C}$ ) ovoid bacterium $1-2 \times 0.8 \mu \mathrm{m}$ with bipolar staining; $34 \mathrm{O}$-antigens and $19 \mathrm{H}$-antigens, a great number of serovars.

Source of infection (natural host range): pig, dog, cat (these species are reservoir of the serovar O3), leporids, rodents (Rattus, Ondatra, Castor), wild mammals, bats, birds, reptiles, frogs, molluscs, fish.

Animal disease: colitis in hares and pigs, in young animals sometimes septicaemia.

Transmission mode: alimentary (replicates in meat and in tins at temperature of $4^{\circ} \mathrm{C}$ - contrary to salmonellae and campylobacters; milk, water); blood transfusion (at least 17 fatalities have been described).

Human disease: yersiniosis - peracute gastroenteritis (main serovar O3, less often O8 [US $\bar{A}$ ] and O9) usually in winter period; pseudoappendicitis in $>10 \%$ cases, fever, sometimes a subsequent polyarthritis, Reiter syndrome, erythema; rarely septicaemia (1\%). For instance, an outbreak due to contaminated pork brawn occurred in Norway, 2005. 
Bio-containment: BSL-2.

Diagnosis: cultivation (faeces, pus from lymph nodes: selective media - e.g. CIN), serotypization, serology (AR, ELISA: serovar O9 of Y. enterocolitica cross-reacts with brucellae).

Treatment: in septical forms tetracycline, cotrimoxazole, doxycycline, chloramphenicol, streptomycin (but not penicillin).

Geographical distribution: largely holarctic, especially in the colder climatic zone (Scandinavia); incidence in Europe is moderately increasing.

\section{Yersinia pseudotuberculosis}

Slowly motile (at $22-25^{\circ} \mathrm{C}$, but not at $37^{\circ} \mathrm{C}$ ) ovoid to short rod $0.6-1.2 \times 0.4-0.8 \mu \mathrm{m}$ with bipolar staining; 10 serovars.

Source of infection (natural host range): cat (carrier host), rodents (reservoir), leporids, dog, beaver (Canada), ruminants, bats, birds; also soil, water (it can replicate in water at $18-20^{\circ} \mathrm{C}$ ), foods, vegetables (Y. pseudotuberculosis replicates in vegetables at $\left.4^{\circ} \mathrm{C}\right)$.

Animal disease: pseudotuberculosis (necroses and granulomas on liver, spleen, lymphatic nodes), especially in rabbit, sheep, birds; cattle, horse, dog.

Transmission mode: alimentary, less often direct contact, via conjunctivae and aerogenic; the agent has considerable tenacity in external milieu.

Human disease: pseudotuberculosis - fever, purpura, acute gastroenteritis, abdominal pain, mesenteric lymphadenitis, hepatosplenomegaly and abscesses in liver, sometimes pseudoappendicitis, bronchopneumonia; occasionally severe invasive septical illness with a considerable mortality.

Bio-containment: BSL-2.

Diagnosis: cultivation of the blood and pus from lymph nodes (at $22-25^{\circ} \mathrm{C}$ ), serology (AR), intradermal test.

Treatment: only in severe forms - ampicillin, tetracycline, streptomycin.

Geographical distribution: worldwide.

\section{Yersinia pestis}

Short Gram-negative rods $1.5 \times 0.5-0.7 \mu \mathrm{m}$ with bipolar staining, pleomorphic (occasionally thread-like, large spherical and other involution forms), non-motile, producing a capsula. A facultative intracellular parasite. The whole genome of $Y$. pestis was sequenced in 2001: it contains 4.7 million nucleotides and 3 plasmids sized 96, 70, and $10 \mathrm{kbp}$. According to phylogenetic analyses $Y$. pestis originated from $Y$. pseudotuberculosis roughly 10,000 years ago as a clone capable of transmission by fleas and lost about $13 \%$ of the original genes. The biovars of $Y$. pestis Antiqua, Medievalis, Orientalis, and Microtus differ in the ability of nitrate reduction, and glycerol and arabinose fermentation. 
Source of infection (natural host range): rodents (reservoir) - in the natural cycle gerbils and jirds (Rhombomys opimus in central Asia; Meriones libycus in north Africa and Arabia; Tatera spp.), marmots in high mountain steppes of Asia and North America (reservoir - Marmota sibirica in Mongolia, M. himalayana in China), pikas Ochotona pallasi and O. daurica in Mongolia, chipmunks Tamias spp. in California, ground squirrels (Spermophilus spp., e.g. S. beecheyi in California, S. undulatus and S. erythrogenys in Mongolia) and prairie dogs (Cynomys in USA); in the secondary urban cycle rats (Bandicota, Mastomys, Rattus, Arvicanthis); Y. pestis is able to survive for long periods in the soil of the rodent burrows (for at least 1 month) and in carrions of mammals (including man). In North America the source of human infection is occasionally cat that hunted infected rodents in a natural focus.

Animal disease: sometimes latent; but more often a general illness, e.g. in brown rats and other rodents (MID in them is about 10 cells), cats (may be severely affected), goats and camels.

Transmission mode (Fig. 8.21): fleas of the genera Xenopsylla (especially $X$. cheopsis, that after death of its rat host easily moves to man as an alternative host; also X. brasiliensis), Oropsylla (O. montana in California, the marmot flea O. silantiewi in Mongolia), Monopsyllus, Nosopsyllus (China), possibly also the human flea Pulex irritans (Tanzania). Bacteraemia in mammalian donors of agent should be at least $10^{8}$ cells $/ \mathrm{ml}$ blood. The imbibed plague bacilli replicate and form a "block" in the flea's proventriculus that

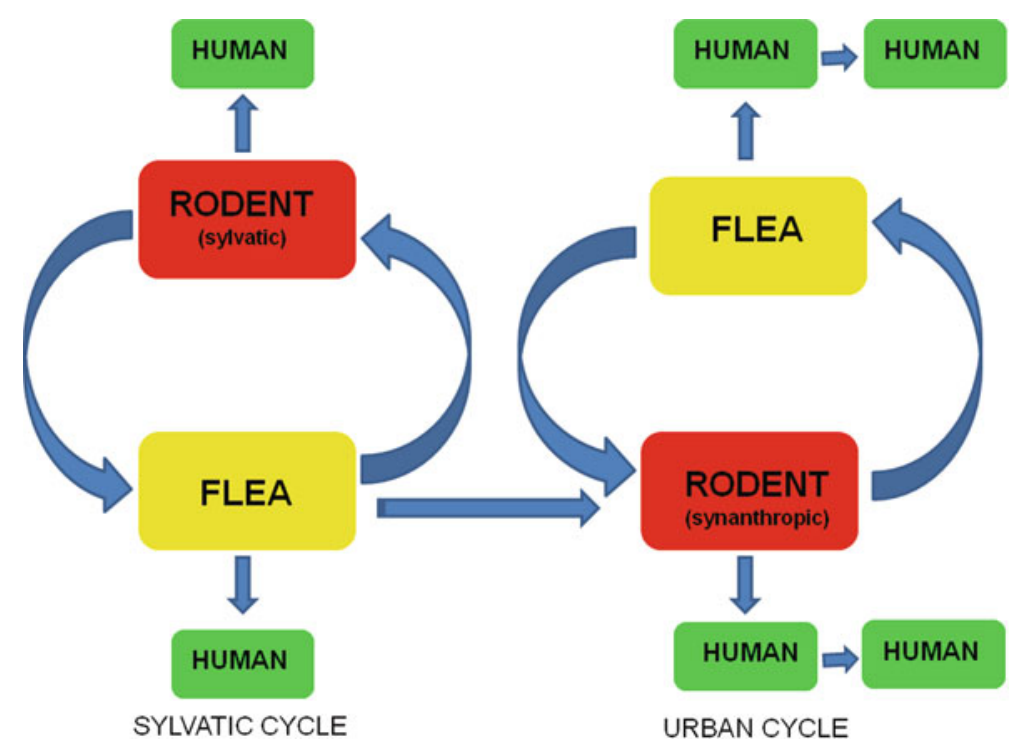

Fig. 8.21 The cycles of Yersinia pestis (drawing by Ivo Rudolf) 
limits the ability of the flea of further blood feeding (Fig. 6.14), and leads to regurgitation. TOT has not been demonstrated in fleas. Other means of plague transmission are aerogenic (person-to-person, droplets during cough) which results in the most feared epidemics, and alimentary - e.g. 18 plague cases were described after consumption of uncooked liver of infected camels in Libya and Saudi Arabia. The plague sometimes occurs as an occupational infectious disease - hunters of marmots and other rodents: e.g. 19 marmot hunters (handling infected animals or their carcasses) got plague in north China in 2004, and 8 died. An alternative (but speculative) mode for Y. pestis transmission during outbreaks might be via the body lice Pediculus humanus.

Types of disease foci: (1) primary, natural, sylvatic (the hosts of the agent are exoanthropic rodents) in steppe, semidesert or tropical forest ecosystems; (2) secondary, urban - largely in sea ports (the main hosts of the agent are rats "urban" plague).

Human disease: plague (pestis) starting with fever and fatigue. The clinical forms are bubonic (bubonic plague, with lymphadenitis: swollen and extremely painful lymph nodes called buboes), pulmonary (severe bronchopneumonia and respiratory distress syndrome - usually in the anthroponotic infection), oroglandular (after an alimentary infection, associated with abdominal pain and diarrhoea), and primarily septic (without lymphadenitis, but with meningitis, endotoxic shock, organ failure and a disseminated intravascular coagulation); occasional severe peripheral tissue necrosis and gangrene remind of medieval epithet ("Black Death"). Mean fatality rate is $2-10 \%$ in treated and $50-95 \%$ in untreated cases (the most lethal are septic and pulmonary forms). Historically, the first major epidemic of plague was reported in China in 224 BC. Later on, three pandemics of plague were recorded: (1) "Justinian's plague" in the sixth century (with roughly 100 million victims out of the 142 million infected); (2) "black death" in the fourteenth century killed nearly whole populations of Tatars and Saracens, then the disease encompassed nearly whole Europe where died one-fourth of the population (about 25 millions), and further roughly 25 millions died before in Asia and Africa; (3) "modern" plague in the nineteenth and twentieth centuries (30 million people got the disease, 12 millions of them died; the pandemic started in Hongkong after introduction from the continental China; rats and their fleas dispersed plague on ships to many seaports of Japan, India, Europe, Africa, America and Australia). According to new molecular data, probably only the biovar Orientalis of $Y$. pestis participated in all three pandemics. The last outbreak of pulmonary plague was recorded in Los Angeles in 1924, with 32 cases ( 30 were fatal). In India, there were 52 fatal cases of plague in 1994. Another outbreak with 18 cases (the bubonic form) occurred in Oran (Algeria) in 2003 (here after more than 50 years). Sporadic cases of plague still occur in a limited number of natural foci in the world. For instance, on average 10 human cases are reported annually from western USA. However according to WHO, there are 1,000-2,500 cases of plague reported each year worldwide; for instance, a total incidence of plague in 
the years 1987-2001 was 36,876 persons, and 2,847 (7.7\%) died. Recent outbreak of plague in Peru (2010) involved 31 cases including 3 deaths (the last outbreak of bubonic plague in northern Peru before was in 1994, which affected more than 1,100 persons and killed 35 of them).

Bio-containment: BSL-3.

Diagnosis: microscopy (methylene blue) and cultivation (BA, agar with gentianviolet at $27^{\circ} \mathrm{C}$ ) of the pus from enlarged lymph nodes, blood, CSF, bone marrow or sputum samples, subcutaneous inoculation of guinea pig, laboratory rat or mouse; serology (IFA, CFT, RIHA).

Treatment: streptomycin, tetracycline, doxycycline, chloramphenicol, gentamicin.

Prevention: vaccine (inactivated or attenuated - strain EV76 produced in the former Soviet Union, or heat-killed Y. pestis vaccine manufactured in Australia) produces a short-term protection; rat control in endemic areas.

Geographical distribution: natural foci of plague occur in parts of Asia (India - Photo 5.39, Burma, Iraq, central Asia, Mongolia - Photo 5.40, northern China), Africa (including Madagascar - here recently $45 \%$ of all African cases), South America and western North America (USA: Oregon, California, Nevada, Arizona, Colorado, Utah, New Mexico, and Texas).

\subsubsection{Family Pasteurellaceae [Order Pasteurellales, Class Gammaproteobacteria]}

\section{Pasteurella multocida, Mannheimia haemolytica}

Short Gram-negative rods to coccobacilli $0.5-1.2 \times 0.3-0.6 \mu \mathrm{m}$ with a marked bipolar staining, facultatively anaerobic, non-motile, forming capsula; serovars A, B, D, E in P. multocida.

Source of infection (natural host range): serovar A birds, serovars B and $\mathrm{D}$ domestic (cattle, sheep, pig, dog, cat, rabbit) and wild (rodents, hare) mammals.

Animal disease: pasteurellosis, called avian cholera in fowl - bronchopneumonia, haemorrhagic septicaemia. Asymptomatic in cats and dogs.

Transmission mode: contact (percutaneous: biting or scratching by a cat, less often a dog; insect bite or sting), sporadically alimentary or aerogenically.

Human disease: pasteurellosis (infrequent illness) - local inflammation, abscesses, lymphadenitis; respiratory illness; sometimes meningitis; rarely appendicitis.

Bio-containment: BSL-2.

Diagnosis: cultivation of pus or tissue samples (BA; does not grow on Endo agar), microscopy of the blood or tissue smears (methylene blue: bipolar staining; IF microscopy), inoculation of mouse or rabbit. 
Treatment: penicillin (higher doses); ceftriaxone, ciprofloxacin, amoxicilin, doxycycline, tetracycline, chloramphenicol, erythromycin, cotrimoxazole, cefuroxim.

Geographical distribution: worldwide, sporadic.

\subsubsection{Family Vibrionaceae [Order Vibrionales, Class Gammaproteobacteria]}

\section{(*) Vibrio cholerae}

Short motile (monotricha) slightly crescent-shaped Gram-negative rods; facultatively anaerobic; one $\mathrm{H}$ antigen, but 80 somatic $\mathrm{O}$-antigens. The serogroups pathogenic for man are O1 (with two biotypes: classical, and haemolytic "El Tor"; and with two main serotypes - Inaba and Ogawa), and O139 (Bengal); infrequently certain others called "non-O1/O139 V. cholerae".

Source of infection: man; brackish water and marine animals (crustaceans mainly Copepoda, shellfish including raw oysters, and fish) in endemic areas.

Transmission mode: alimentary (municipal or surface water, food); $V$. cholerae non-O1 quite often after consumption of raw "seafood" or street vendors' beverage. The agent survives in water for up to several weeks.

Human disease: cholera - sudden vomiting without nausea, intense longterm watery diarrhoea (excrements look like rice water and have fish odour) causing rapid dehydratation and hypovolemic shock, anuria, muscle cramps; death can occur in severe cases sometimes within 1 or 2 days. The cholera enterotoxin stimulates hyperproduction of cAMP that inhibits in the cells of intestinal villi influx of ions $\mathrm{Na}+$ and $\mathrm{Cl}-$ and at the same time stimulates hypersecretion of $\mathrm{Cl}$-and $\mathrm{HCO}_{3}$ - causing a blockade of intestinal water influx. V. cholerae non-O1 causes less severe but sometimes bloody diarrhoea and abdominal spasms. A total of 10 explosive pandemics of cholera occurred in the nineteenth and twentieth century, and most of them were initiated in the Indian subcontinent. For instance the first known pandemic of cholera (1817) started in the Gangus river valley, and spread up to southern Russia. A new serotype O139 Bengal caused first outbreak in Bangladesh in 1992, and reemerged there in 2002 (about 30,000 cases). The risk of cholera always increases during great humanitarian disasters, civil wars, in refugee camps. [Extensive anthroponotic epidemics in Zimbabwe and Tanzania occurred in 2008-2009].

Bio-containment: BSL-2.

Diagnosis: microscopy (darkfield) and cultivation of faecal samples (enrichment in alkalic peptone water and a subsequent isolation on agar medium TCBS with thiosulphate, citrate, deoxycholate and saccharose), combined with agglutination of the grown colonies. 
Prevention: inactivated oral vaccines (DUKORAL, OROCHOL); the use of packaged water in endemic areas, appropriate hygiene practices.

Treatment: intense rehydration and influx of ions; antibiotics (gentamicin, chloramphenicol, tetracycline).

Geographical distribution: tropics and subtropics. Endemic area is, e.g., Bangladesh.

\section{*Vibrio parahaemolyticus}

A halophilic marine bacterium, pathogen of fish. Pandemic strain (spreading since 1996) posses antigenic structure O3:K6.

Source of infection: marine coastal water (especially at higher temperatures, often coincidence with algal overpopulation), marine fish, crabs and oysters.

Transmission mode: alimentary ("seafood-borne disease": oysters etc.). In addition, asymptomatic human carriers of these strains have been revealed.

Human disease: vibrio gastroenteritis - an about 3-day, often bloody, diarrhoea, abdominal pains, nausea, vomiting. In Japan the agent causes about $50 \%$ of all patients with bacterial gastroenteritis. Sometimes epidemic occurrence. For instance, during a recent outbreak in Chile (2004-2007), the total number of reported cases was 10,783 (as of March 2005), making this the largest documented occurrence of $V$. parahaemolyticus diarrhoea in the world. The bacterium also causes ear and wound infections.

Bio-containment: BSL-2.

Diagnosis: cultivation of stools and food remnants (TCBS agar with 3\% $\mathrm{NaCl}$ ); serotypization, Kanagawa's test for detection of thermostable haemolysin.

Treatment: rehydration.

Geographical distribution: worldwide - coastal areas, very often in Japan and Singapore, but also other parts of southeast Asia, further India, Spain, USA and South America.

\section{*Vibrio vulnificus}

A halophilic bacterium, serious pathogen of eel.

Source of infection: coastal water, marine fish, crabs and oysters (a halophilic bacterium).

Transmission mode: contact (skin injuries, especially with fish bones), alimentary (e.g., raw oysters, fish).

Human disease: skin wound lesions with necrotizing fasciitis, primary septicaemia sometimes accompanied with a septic shock (fatality rate 30-50\%); or acute diarrhoea (fatality rate low, but in the biotype 3 up to $10 \%$ ). Usually sporadic cases, sometimes epidemics (e.g. in Israel, 1996/1997: 134 cases in fishers and fish consumers, biotype 3 ). In the Los Angeles area (California), a 
total 252 cases were reported between 1980 and 2004, and in Japan 94 cases between 1999 and 2003.

Bio-containment: BSL-2.

Diagnosis: cultivation (TCBS agar, with $3 \% \mathrm{NaCl}$ ).

Treatment: rehydration in intestinal form of the disease, antibiotics in severe cases (cephotaxin + minocycline); surgery (amputation occasionally necessary in fasciitis cases).

Geographical distribution: Mexico, southern USA, India, southeastern Asia (Taiwan), Japan, Israel, Baltic Sea, Spain.

\section{*Vibrio metschnikovii}

Source of infection: coastal marine or brackish water, fish, oysters (a halophilic microorganism).

Animal disease: pathogenic for birds, illness in birds similar to cholera.

Transmission mode: contact, alimentary.

Human disease: diarrhoea, pneumonia, infection of wounds etc. - low incidence.

Bio-containment: BSL-2.

Geographical distribution: southern USA, Southeast Asia, Germany.

\section{*Other "Non-cholera" Vibrios}

Pathogenic for man (causing wound infections or gastroenteritis) but with a lower risk, they involve Vibrio mimicus, V. fluvialis, V. furnissii, V. alginolyticus, Grimontia hollisae, and Photobacterium damselae.

\subsubsection{Family Aeromonadaceae [Order Aeromonadales]}

\section{*Aeromonas hydrophila, A. caviae}

Gramnegative rods to coccobacilli, facultatively anaerobic, usually motile (with one terminal flagellum). Some human isolates posses Shiga toxin genes (stx1, stx2) similar to those in pathogenic Escherichia coli strains.

Animal disease: pathogenic for frogs and fish.

Source of infection: water.

Transmission mode: contact, alimentary (e.g., fish). Sometimes associated with disasters (hurricanes, tsunami, earthquakes).

Human disease: gastroenteritis, bacteraemia, prostatitis, HUS. Usually sporadic cases.

Bio-containment: BSL-2.

Diagnosis: cultivation and biochemical tests.

Treatment: antibiotics in severe cases.

Geographical distribution: worldwide. 


\subsubsection{Family Campylobacteraceae [Order Campylobacterales, Class Epsilonproteobacteria]}

\section{Campylobacter jejuni (C. coli, C. laridis, C. foetus)}

Mildly bended, S-shaped to shortly screw-like Gram-negative rods $0.5-1.2 \times 0.2-$ $0.5 \mu \mathrm{m}$ with two polar flagella (amphitricha), aerobic to microaerophilic, thermophilic (optimum growth temperature $42^{\circ} \mathrm{C}$, do not grow at $<30^{\circ} \mathrm{C}$ ); 65 serovars differentiated according to $\mathrm{O}$ and $\mathrm{H}$ antigens.

Source of infection (natural host range): domestic fowl ( $>50 \%$ human cases; in broilers commonly up to $10^{7}$ cells/g) and other birds (reservoir), mammals (cattle, sheep, pig - C. coli, dog, cat); man (about $1 \%$ asymptomatic carriers); oysters, crabs; surface water (contaminated by birds).

Animal disease: usually asymptomatic, or diarrhoea (calves); fowl hepatitis. $C$. foetus causes septic abortions and fertility disturbances in sheep and cows. Transmission mode: alimentary - e.g. grilled fowl (especially chicken broilers), hamburgers ("fast-food"), processing raw meat, drinking raw milk, eating fresh cheese, water (campylobacters survive in it for $>4$ months $/ 5^{\circ} \mathrm{C}$ ); contact. Mechanical transmission by flies has been repeatedly recorded. MID for man is 500-800 cells.

Human disease: campylobacterosis (commonly used term "campylobacteriosis" is imprecise because the agent is not a "Campylobacterium", but Campylobacter), together with salmonellosis probably the most frequent zoonosis and a major health problem worldwide. After a short incubation period (usually 3-5 days) appears fever (with chills), headache, myalgia, anorexia, nausea, acute enteritis (abdominal pains are stronger than in salmonellosis), prolonged diarrhoea (sometimes bloody) for up to 10 days, pseudoappendicitis, arthritis; sometimes meningoencephalitis (C. foetus). The arthritic and neurologic sequelae of infection with $C$. jejuni involve in $<1 \%$ of patients Guillain-Barré syndrome (demyelinizing peripheral neuropathy with acute neuromuscular paralysis: symmetrical flaccid effect on limbs and loss of tendon reflexes; up to $30 \%$ cases of this polyradiculoneuritis syndrome is attributed to $C$. jejuni) or Reiter syndrome (reactive arthropathy); mortality is rare. Campylobacterosis, in contrast to salmonellosis, occurs more often sporadically than in epidemics. Incidence rate was 20-110 per 100,000 population in Europe in 1996-1999, while 7-32 in the USA (that of salmonellosis was 13-26 in the same period). In Great Britain the annual incidence of campylobacterosis attained 50,000 cases in the years 1997-1999 and surpassed that of salmonellosis (51,800 cases in 2007). A similar trend was observed in Czechland (EPIDAT Report on incidence of infectious diseases): while the mean annual incidence was 8,630 cases in the decade 1990-2000, as many as 23,480 cases (annual average; the range, 20-30 thousands) were reported in the period 2001-2008, i.e. not much 
less than in salmonellosis in the same period (annual average 25,800, the range 11,000-33,000 cases). However, this obviously increasing trend could be biased by a much better and more widespread laboratory diagnosis of campylobacterosis.

Bio-containment: BSL-2.

Diagnosis: microscopy of native stool samples in darkfield, cultivation of faeces (sampled in a special transport medium), blood or CSF on selective Preston agar with charcoal and cefoperazon or on other special agar media (Karmali; Butzler) at $42^{\circ} \mathrm{C}$ in $5-10 \% \mathrm{CO}_{2}$ and $85 \% \mathrm{~N}_{2}$; serology (ELISA, CFT), PCR. Treatment: rehydration; antibiotics should be used only in severe cases (erythromycin, tetracycline, azithromycin, clarithromycin, ciprofloxacin). Campylobacters are usually resistant to quinolones (these drugs used to be occasionally added to chicken food).

Geographical distribution: worldwide.

\subsubsection{Family Helicobacteraceae [Order Campylobacterales]}

\section{Helicobacter bizzozeronii, $\mathrm{H}$. felis}

Small, mildly bended and motile Gram-negative rods, microaerophilic. In the past, helicobacters were classified in the genus Campylobacter, and the two mentioned species formed a group Helicobacter "heilmannii", observed in animals and man. [Anthroponotic species is H. pylori, causing gastritis and peptic ulceration].

Source of infection (natural host range): $\operatorname{dog}$ (H. bizzozeronii), cat (H. felis), pig.

Animal disease: probably usually asymptomatic.

Transmission mode: alimentary or contact.

Human disease: gastritis, severe dyspeptic symptoms. Only few cases have been described (e.g., one in Finland recently).

Bio-containment: BSL-2.

Diagnosis: microscopy of native stool specimens in darkfield, cultivation from faeces (very difficult).

Treatment: unknown (metronidazole unsuccessful).

Geographical distribution: Europe, possibly worldwide.

\subsubsection{Family Leptospiraceae [Order Spirochaetales, Class Spirochaetes]}

In addition to saprophytic leptospirae (e.g., L. biflexa, L. parva) living in water, 13 spp. are regarded as pathogenic, with more than 260 serovars at present. However, the most important is one species $-L$. interrogans. 


\section{Leptospira interrogans}

A very motile obligately aerobic spirochaete $6-20 \times 0.1-0.2 \mu \mathrm{m}$ with many dense coils, two periplasmic flagella and usually hooked ends. Twenty-three serogroups with more than 200 serovars have been described in this species.

Source of infection (reservoirs) with particular serovars: brown rat (Icterohaemorrhagiae), voles and other rodents (Grippotyphosa, e.g., Microtus agrestis, $M$. arvalis: persistent infection in these species, the agent lives in renal tubules and is excreted in urine that contains up to 106 leptospirae per ml) and Sejroe (largely Mus musculus); pig (Pomona - also Apodemus agrarius, and $\overline{\text { Australis })}$, cattle (Tarassovi, Hardjoe), dog (Canicola), hedgehog and horse (Bratislava). Leptospirae are very resistant in water milieu; they can also be transported by waterbirds (from and to rice fields etc.).

Animal disease: inapparent, or (cattle, pig) abortions, meningitis and dying of some young animals.

Transmission mode: contact (percutaneous; bathing, water sports, work in water or in sewers), alimentary (water or food contaminated with rodent urine, e.g. after floods; uncooked meat of infected animals).

Human disease: leptospirosis is one of the most widespread zoonoses in the world - a flu-like illness, often with biphasic body temperature curve, aseptic meningitis and nephritis (albuminuria). The course of the disease varies according to serovar; the most severe is Weil's disease (L. interrogans Icterohaemorrhagiae) with hepatitis, icterus, myalgia, necroses, cardiac and ocular involvement, nephritis (damage to glomerulae; leptospiruria), haemorrhagies, encephalitis, and fatality rate in untreated cases 10-20\%; milder but most frequent is the "harvest fever" in central Europe (caused by L. interrogans Grippotyphosa); also other anicteric leptospiroses usually have milder symptoms, e.g. "fever of swine breeders" (L. interrogans Pomona). The incidence of leptospirosis is not very high in most countries; for instance in Czechland, on the average 82 (the range, 12-226) cases were reported annually in the years 1970-1999, while 38 (the range, 11-88) in the decade 1990-2000 (0.4 cases per 100,000 population); in France, an average of about 250 cases annually have been reported since 1980 (0.5 cases/100,000). Occupational disease: up to $30 \%$ of cases form butchers in slaughterhouses, farmers, meat inspectors, workers in sewerage system, miners, soldiers, gamekeepers, hunters, rodent control workers, sugar cane cutters, ricefield workers, animal attendants in zoological gardens and workers in sewerage system; leptospirosis also often occurs as a "recreational" (leisure time) infectious disease (about 30\% of all cases) - during outdoor activities like water sports (canoeing, swimming), or camping. Epidemics also follow natural disasters, such as floods, hurricanes or earthquakes. For instance, 167 Philippinos died from leptospirosis after storm Ondoy flooded Manila and Rizal in 2009.

Bio-containment: BSL-2. 
Diagnosis: microscopy (darkfield or phase contrast) and cultivation (EMJH [Ellinghausen-McCullough-Johnson-Harris] medium, Korthoff's fluid peptone medium supplemented with rabbit serum or bovine albumin and Tween 80, or Fletcher, Noguchi and Stuart liquid media; however, the growth of leptospirae is slow) of the blood (acute stadium) or urine (convalescence) samples; intraperitoneal inoculation of young guinea pig or hamster; serology (CFT, slide AR, IHA, ELISA; the most specific is the microagglutination-lysis test, when a viable culture is mixed with antiserum, and then observed under darkfield microscope); RFLP, ribotyping, PFGE.

Treatment: penicillin, ampicillin, doxycycline, tetracycline, streptomycin, erythromycin, ceftriaxone, cefotaxime, ciprofloxacin; haemodialysis is sometimes necessary during renal insufficiency.

Prevention: vaccination of domestic animals (cattle, pigs, dogs) is possible with serovar-specific vaccines. Vaccines for humans are produced in France (serovar Icterohaemorrhagiae), China and Japan (serovars Canicola, Icterohaemorrhagiae and Pomona).

Geographical distribution: worldwide (mainly humid ecosystems: Photo 5.38).

\subsubsection{Family Spirochaetaceae [Order Spirochaetales]}

\section{${ }^{* *}$ Borrelia recurrentis}

A spirochaete up to $30 \mu \mathrm{m}$ long, genomically very closely related to $B$. duttonii as found by DNA sequencing and molecular phylogenetic analyses. In fact, it should be regarded as a population subset or an ecotype of $B$. duttonii rather than a separate species.

Source of infection (natural host range): man.

Transmission mode: body louse (Pediculus humanus; the lice, when infected, remain infectious longlife) - by swating and rubbing in skin (or conjunctiva).

Human disease: epidemic louse-borne relapsing fever (typhus recurrens) - high fever for 3-9 days with 1-5 relapses (they are associated with the change of surface antigens of the spirochaetes and their reappearance in the blood; afebrile intervals use to be 5-10 days), chills, severe headaches, myalgia, arthralgia, abdominal pain, anorexia, hepatosplenomegaly, sometimes icterus, CNS affection (photophobia, dizziness); fatality rate in untreated cases up to $40 \%$, in treated patients $4-7 \%$. The spirochaetes in the human blood first observed and described Obermaier in 1867. Epidemic of recurrent typhus (on many occasions together with rickettsial epidemic typhus) are determined by socioeconomic factors, they occur during wars, famine and big disasters, usually in winter and early spring. Examples: in 1919-1923, epidemics in southern Europe (the Balkans) and Russia (5 million victims); recent cases and outbreaks have been reported in Ethiopia (1,000-5,000 cases annually), Sudan, Burundi, Rwanda, Uganda, China, Peru, and Russia. 
Bio-containment: BSL-2.

Diagnosis: darkfield microscopy of the blood (in the acute phase patients have up to 106 spirochaetes/ml blood), blood smear or thick drop (stained by Giemsa, IF); inoculation on suckling mice or chick embryo; cultivation in vitro is difficult (fluid BSK medium); serology (Weil-Felix reaction with antigen Proteus OXK).

Treatment: tetracycline, erythromycin, doxycycline, penicillin.

Geographical distribution: Africa (Ethiopia, Rwanda, Senegal), Asia Minor and Central Asia, foothills of the Andes.

\section{Borrelia duttonii, B. hispanica, B. crocidurae, B. persica, B. caucasica, B. latyschevi, B. hermsii, B. parkeri, B. turicatae, B. theileri, B. coriaceae, B. venezuelensis, B. mazzottii, B. miyamotoi}

A large group of relapsing fever zoonotic borreliae. B. duttonii is phylogenetically closely related to $B$. recurrentis (even more than to $B$. hispanica) and B. crocidurae.

Source of infection (natural host range): rodents (mice, hamsters, gerbils), porcupine, hedgehogs, canids and other wild mammals (armadillo, opossum, bats), and some reptiles (agama); man (B. duttonii).

Animal disease: usually inapparent course (arthritis in dogs).

Transmission mode: argasid ticks of the genus Ornithodoros (reservoir - TOT), living in burrows of medium-sized rodents and other mammals, in small caves, crevices of cottages and buildings: O. moubata (B. duttonii in eastern Africa), O. marocanus (B. hispanica), O. sonrai (B. crocidurae in western Africa), O. erraticus (B. crocidurae, B. hispanica), O. tholozani and $O$. papilidog (B. persica), O. verrucosus (B. caucasica), O. hermsi (B. hermsii), O. turicata (B. turicatae), O. rudis (B. venezuelensis), O. parkeri (B. parkeri), O. coriaceus (B. coriaceae), O. rudis (B. venezuelensis), O. tartakovskyi (B. latyschevi), O. talaje (B. mazzottii) - by blood feeding, also via coxal fluid of the soft ticks. Sometimes transmit the borreliae also ixodid ticks: Boophilus microplus (B. theileri), Ixodes persulcatus, I. ricinus and I. scapularis (B. miyamotoi).

Natural cycle: mammal $\rightarrow$ argasid $\rightarrow$ mammal.

Human disease: endemic tick-borne relapsing fever (endemic recurrens) papula, rash (in half of patients), recurrent fever with chills (2-10 relapses going on for 2-7 days as a reaction to the antigenic "shift" of the agent, afebrile periods last 2-5 days or up to several weeks), hyperhidrosis, headaches, myalgia, arthralgia, abdominal pain, cough, conjunctivitis, lymphadenopathy, miscarriage, in a part of patients also affection of CNS, myocarditis, hepatosplenomegaly; fatality rate $2-10 \%$.

Bio-containment: BSL-2.

Diagnosis: darkfield microscopy of the blood smear or a thick drop from the febrile phase (Giemsa or Wright staining, IF); intranasal (or conjunctival) 
inoculation of guinea pig or intraperitoneal inoculation of suckling mouse; serology (CFT), detection of the antigen (immunohistochemistry), cultivation (BSK medium).

Treatment: penicillin, tetracycline, erythromycin, doxycycline, chloramphenicol.

Geographical distribution: subtropics and tropics (steppe to semidesert habitats) - the Mediterranean (Pyrenean Peninsula, Greece), North Africa (B. hispanica is frequent in Morocco), tropical east Africa (Tanzania etc.), western Africa (B. crocidurae - Senegal), the Caucasus, the Near East, Central Asia (B. latyschevi - Tadjikistan, Uzbekistan), India, China, Japan, America (B. parkeri - Colorado, B. hermsii, B. turicatae, B. coriceae), Mexico (B. mazzottii).

\section{Borrelia lonestari}

A spirochaete belonging to the group of relapsing fever zoonotic borreliae.

Source of infection (natural host range): rodents.

Animal disease: not observed.

Transmission mode: bites of the Lone Star tick Amblyomma americanum.

Human disease: Southern tick associated $\underline{\text { rash }} \underline{\text { illness }}$ (STARI) - similar to erythema migrans in Lyme borreliosis (see below).

Bio-containment: BSL-2.

Diagnosis: similar as in the previous borreliae, but cultivation in vitro is difficult.

Treatment: antibiotics.

Geographical distribution: southern USA.

\section{Borrelia burgdorferi s.l.}

Spirochaetes 4-25 $\times 0.2-0.3 \mu \mathrm{m}$ with 3-10 coils and 7-11 periplasmic flagella. Genomic species ("genomovars", called often "genospecies" in discrepancy with standard bacteriological nomenclature) of B. burgdorferi s.l. (described as late as 1982), pathogenic for man, are B. burdorferi s.s. (its full genome was already sequenced), B. afzelii and B. garinii, less often B. valaisiana, B. lusitaniae, while genomic species with not yet sufficiently demonstrated pathogenicity are $B$. bissetii, B. spielmanii and a few others. In the host, some borreliae are able to produce persisting 'cystic forms' or 'blebs'.

Source of infection (natural host range): forest rodents (in Eurasia Apodemus and Myodes spp. - competent hosts and the reservoir especially for B. afzelii; in America Peromyscus leucopus), squirrels, chipmunks (e.g., Tamias stria$t u s$ ), leporids, turdid and other ground-foraging forest birds (the reservoir for B. garinii); also lizards (B. lusitaniae). 
Animal disease (dog, ruminants, horse): borreliosis with arthritis, affection of the kidneys and CNS.

Transmission mode: ixodid ticks of the Ixodes ricinus complex (reservoir TOT, TST): I. ricinus, I. persulcatus, I. pavlovskyi, I. scapularis, I. pacificus, also I. uriae (B. garinii, in seabird colonies: Photos 5.37, 7.77).

Human disease: Lyme borreliosis (LB, or Lyme disease in America, described in Old Lyme, Connecticut, in 1975) - a multisystem illness with 3 clinical phases (the course of untreated LB can remind that of the syphilis): (1) in 60$90 \%$ of infected persons appears a localized erythema (erythema migrans, EM) that was already described by Afzelius (1910) and Lipschütz (1913); (2) when not treated with antibiotics, the spirochaetes disseminate and secondary lesions may appear such as new EM lesions or lymphocytoma (lymphadenosis benigna cutis), sometimes increased temperature, headaches and myalgia, fatigue, regional lymphadenopathy, conjunctivitis, affection of CNS (meningitis), polyneuritis ( $n$. facialis - Bell's palsy, radiculoneuritis), arthralgia and carditis; (3) the late phase (usually after 2-3 years) is manifested by chronic relapsing polyarthritis affecting big joints (synovitis, erosion of cartilage), chronic neuroborreliosis (peripheral neuropathies, affection of CNS), chronic affection of the skin (acrodermatitis chronica atrophicans, ACA: described by Buchwald already in 1883). B. burgdorferi can sometimes persist in the human body for long periods (up to 10 years), e.g. in synovial fluid or in the CNS (often in changed morphological forms - cystic, circular or granular). $\mathrm{LB}$ is the most frequent ixodid tick-borne human disease in the world, with an estimated 85,000 patients annually (Europe 65,000, North America 16,500, Asia 3,500). For instance in Czechland was reported on average 3,330 (the range, 1,446-6,302) cases annually in the years 1990-2006, which presents an incidence rate of 32 (the range, 16-61) per 100,000 population (EPIDAT).

Bio-containment: BSL-2.

Diagnosis: symptoms (EM) and anamnesis (tick bites), serology (ELISA IgM and IgG, optimally with confirmation using WB; IF); isolation of spirochaetes from the skin biopsy or from CSF fluid complex BSK (or MPK) medium at $33^{\circ} \mathrm{C}$ (the growth is very slow); also electron or IF microscopy of CSF; PCR. The 'cystic forms' or 'blebs' are impossible to diagnose in darkfield microscopy.

Treatment: doxycycline, amoxicilin, penicillin, tetracycline, erythromycin, deoxymykoin in the acute phase; in the chronic phase cephalosporins (cefotaxim, ceftriaxon), or big doses of penicillin.

Prevention: suitable human vaccine against LB does not exist at present. The lately released US vaccine "LYMErix" was removed from the market several years ago. Examination by darkfield microscopy or PCR of ixodid ticks feeding on a person may be helpful - in positive cases, prophylactic treatment of the person with one or few doses of doxycycline should be recommended.

Geographical distribution: holarctic (Europe; northern Asia - including Siberia, China, Japan, Korea; and North America). For different habitats of LB agents, see Photos 5.33 to 5.37 . 


\subsubsection{Family Serpulinaceae [Order Spirochaetales]}

\section{Brachyspira pilosicoli}

An anaerobic spirochaete.

Source of infection (natural host range): pig - some strains of swine brachyspirae are pathogenic for man.

Animal disease: enteritis.

Transmission mode: contact, alimentary.

Human disease: enteritis, intestinal spirochaetosis (colon epithelium is affected) - abdominal pain, chronic (sometimes bloody) diarrhoea; the illness occurs mainly in immunosuppressed persons. In Bangladesh, often associated with cholera. Occupational risk (butchers, vets, animal breeders).

Bio-containment: BSL-2.

Diagnosis: cultivation in vitro is difficult.

Treatment: antibiotics (optimally after differential susceptibility test).

Geographical distribution: worldwide.

\subsubsection{Family Flavobacteriaceae [Order Flavobacteriales, Class Flavobacteria]}

\section{Capnocytophaga canimorsus}

Motile (gliding) Gram-negative fusiform rods to filaments, facultatively anaerobic, and fastidious.

Source of infection (natural host range): dog (a commensal in the oral cavity), less often cat, and rodents.

Animal disease: usually asymptomatic infection.

Transmission mode: percutaneous (by bites, scratches, or licking of scratched skin by pet animals).

Human disease: fever, chills, myalgia, vomiting, septicaemia (disseminated) intravascular coagulation, peripheral gangrene, purulent meningitis, endocarditis. Infection can be fatal (sepsis) especially in immunosuppressed individuals. Since the first description in 1976, about 200 human cases have been reported. Risk groups are animal keepers, breeders, veterinarians and pet owners.

Bio-containment: BSL-2.

Diagnosis: cultivation in vitro is difficult (BA or chocolate agar in the atmosphere of $5 \% \mathrm{CO}_{2}$, or anaerobically), the growth is slow; microscopy (bacteria present within neutrophils).

Treatment: penicillin G, doxycycline, imipenem, clindamycin (or other antibiotics optimally after differential susceptibility test). 
Geographical distribution: worldwide (North America, Australia, South Africa, Europe).

\subsubsection{Family Burkholderiaceae [Order Burkholderiales, Class Betaproteobacteria]}

\section{Burkholderia mallei}

A non-motile, slightly bended, strictly aerobic, polymorphic Gram-negative rod 2$5 \times 0.5-1 \mu \mathrm{m}$.

Source of infection (natural host range): equids (horse, donkey, mule), rodents (Rattus), cat, dog; man.

Animal disease: glanders (malleus) - very dangerous, acute (donkey, mule) or also chronic (horse - carriers) infectious illness of odd-toed ungulates, producing nodules and ulcerations in the respiratory tract, occasionally also skin ulcerative lesions called "farcy".

Transmission mode: percutaneous (skin abrasions) or aerogenic, less frequently alimentary, via conjunctiva. The agent is highly contagious, but its tenacity is low.

Human disease: glanders (malleus) - multiple nodules, abscesses or flat ulcers in subcutis, muscles, visceral organs and on mucosa, high fever with headaches, photophobia, lacrimation, lymphadenitis, purulent haemorrhagic bronchopneumonia and septicaemia with a high fatality rate (40-100\%) in untreated cases; less common is a chronic form.

Bio-containment: BSL-3. Considered to be a serious bioterrorist threat (against animals and humans; it was used during the WWI and WWII against military horses).

Diagnosis: microscopy and cultivation (media with 3-5\% glycerol) of the pus from scars and nasal secret; intraperitoneal inoculation of male hamster or guinea pig (scrotal oedema - so-called Strauss effect), serology (CFT, ELISA, RIHA, AR), intradermal test for hypersensitivity (malein), PCR.

Treatment: streptomycin, tetracycline, chloramphenicol, gentamicin; sulphonamides sulphadiazin, cephtazidim, carbapenem.

Geographical distribution: Asia (Mongolia, Turkey, Middle East, Iran, Pakistan), north and eastern Africa, Mexico, South America (Brazil). In the past worldwide, but eradicated from most countries, lately reemerging in some areas (e.g., Arab Peninsula).

\section{*Burkholderia pseudomallei}

A motile polymorphic aerobic rod $1.5-6 \times 0.5-1 \mu \mathrm{m}$ with bipolar staining, growing also at $42^{\circ} \mathrm{C}$. 
Source of infection: stagnant water (in rice fields, wetlands), potable water, soil/mud (a soil-borne bacterium); infrequently it occurs in rodents, sheep, cattle, pig, horse, leporids; occasionally primates, deer, dogs, cats, koalas, kangaroos, camels, crocodiles.

Animal disease: inapparent course, or acute melioidosis (in sheep).

Transmission mode: percutaneous (traumatic inoculation from the soil), less often aerogenic (inhalation of contaminated dust or water droplets, with a severe course) or alimentary (e.g. water ingested or swallowed during swimming); the agent has considerable tenacity in the milieu.

Human disease: melioidosis (pseudomalleus, also called Whitmore disease or Nightcliff gardener's disease in Australia) - fever, a fierce diarrhoea of an extraordinary frequency (20 per h), disseminated disease with multiple abscesses and granulomas (similar to those in tuberculosis) in different organs (liver, lungs, spleen, kidney, prostate) and also in subcutis, lymphadenopathy, pneumonia, sometimes also septicaemia with a very high fatality rate (80-95\%); also chronic forms occur with relapses even after a number of years (despite therapy). It is primarily an infection of humans with underlying diseases such as alcoholism, malnutrition, cirrhosis, and immunosuppression, but can also affect healthy individuals. An increased incidence of melioidosis is usually recorded in endemic areas after cyclones, heavy rains or floods. This is also a "tourist infectious disease" during the travel or adrenaline sports in wet tropical areas (importation to e.g. Europe has been repeatedly recorded).

Bio-containment: BSL-2/3.

Diagnosis: cultivation of the blood, sputum, urine and pus samples, inoculation of young male hamster or guinea pig (scrotal reaction), serology (HA, IFA, CFT, AR, ELISA).

Treatment: long-term (6-12 months) application of doxycycline + amoxicilin, or large doses of sulphonamides (cephtazidim, cotrimoxazole, imipenem, meropenem, carbapenem).

Geographical distribution: tropics and subtropics (largely wetland habitats) Southeast Asia (Singapore, Indonesia) and northern Australia; also China, Taiwan, India, Bangladesh, Iran, Madagascar, Ecuador, Peru, Brazil, and Central America (Caribbean islands).

\section{* Burkholderia cepacia Complex}

About 10 species, pathogenic are mainly B. cepacia, B. multivorans, B. cenocepacia, and B. cocovenenans.

Source of infection: soil, water, hospital environment (nosocomial infectious disease); also man.

Transmission mode: percutaneous, less often aerogenic or alimentary. 
Human disease: bronchitis to pneumonia especially in person with cystic fibrosis and vascular transplantates. B. cocovenenans causes alimentary, often fatal intoxications (2007 Java: 10 patients died due to this illness).

Bio-containment: BSL-2.

Diagnosis: in vitro cultivation of the sputum samples.

Treatment: piperacilin, cotrimoxazole, chloramphenicol, carbopenem.

Geographical distribution: worldwide.

\subsubsection{Family Neisseriaceae [Order Neisseriales, Class Betaproteobacteria]}

\section{*Chromobacterium violaceum}

Gram-negative, facultatively anaerobic rods.

Source of infection: soil and water (saprophytic growth).

Animal disease: pathogenic for birds, their illness reminds cholera.

Transmission mode: contact, alimentary.

Human disease: chromobacteriosis with fever, headache, vomiting, cutaneous, hepatic and pulmonary abscesses, sometimes fatal septicaemia. The illness is infrequent (about 150 cases have been described), but serious.

Bio-containment: BSL-2.

Diagnosis: cultivation - the bacterium produces violet pigment due to tryptofan oxidation on usual agar media.

Treatment: gentamicin, trimethoprim + sulphamethoxazole, meropenem, ciprofloxacin, chloramphenicol.

Geographical distribution: (sub)tropical regions - Asia, Australia, USA, South America (Argentina, Brazil), Africa.

\subsubsection{Family Spirillaceae [Order Nitrosomonadales, Class Betaproteobacteria]}

\section{Spirillum minus}

A short coiled pleomorphic Gram-negative rod 2-5 $\times 0.2-1 \mu \mathrm{m}$, with several (up to 7) polar flagellas (amphitricha); sometimes it forms long and disintegrating filaments.

Source of infection (natural host range): rodents (largely Rattus spp.) - they excrete the agent by saliva.

Animal disease: inapparent course.

Transmission mode: percutaneous (biting). 
Human disease: $\underline{\text { sodoku }}$ [Japanese "so" = black rat; "doku" = poison], rat-bite fever - papular rash (on face and arms), ulceration, regional lymphadenitis, high recurrent fever (usually 6-8 relapses within 2 months, but sometimes prolonged over a number of months or even years), without arthritis; fatality rate $5-10 \%$.

Bio-containment: BSL-2.

Diagnosis: intraperitoneal inoculation of laboratory rat, mouse or guinea pig (bacteraemia); darkfield microscopy, thick drop or blood smear (Giemsa stain); non-cultivable in vitro.

Treatment: penicillin, aureomycin.

Geographical distribution: eastern Asia (Japan), Australia.

\subsubsection{Family Fusobacteriaceae [Order Fusobacteriales, Class Fusobacteria]}

\section{Streptobacillus moniliformis}

A non-motile pleomorphic Gram-negative rod 1-3 $\mu \mathrm{m}$ long, sometimes producing very long and disintegrating filaments up to $100 \mu \mathrm{m}$; facultatively anaerobic.

Source of infection (natural host range): rodents (Rattus etc. - S. moniliformis is a part of their oral microflora; it is excreted via saliva and urine); cat.

Animal disease: usually asymptomatic infection, but sometimes polyarthritis, oedema of limbs and fingers.

Transmission mode: percutaneous (bites); alimentary (ingesting food or drink such as milk or water contaminated with rat excrement).

Human disease: Haverhill fever (rat-bite fever, streptobacillosis) with headache, sometimes rash of rubella type, photophobia, lymphadenitis, pharyngitis, septic mono- or polyarthritis, occasionally endocarditis and pneumonia; fevers can be recurrent for a period of months; fatality rate $10 \%$ in untreated cases.

Bio-containment: BSL-2.

Diagnosis: cultivation of the blood or synovial fluid samples (BA with serum or ascitic fluid, 5-10\% $\mathrm{CO}_{2}$ ), intraperitoneal inoculation of mouse, agglutination.

Treatment: large doses of penicillin, tetracycline (streptomycin).

Geographical distribution: worldwide but sporadic.

\section{Fusobacterium necrophorum}

Anaerobic non-motile Gram-negative rods $2-5 \times 0.5-1.5 \mu \mathrm{m}$ or filaments up to $80-$ $100 \mu \mathrm{m}$ long.

Source of infection (natural host range): domestic ruminants. 
Animal disease: necrobacillosis (necrotic lesions on limbs, diphteroid stomatitis, arthritis, abscesses on liver) in calves, lambs, piglets and young rabbits, with a high fatality rate; necrosis of genital organs in male bisons (Bialowieža, Poland).

Transmission mode: contact; tenacity of the agent is low.

Human disease: necrobacillosis - necrotic ulcers and regional lymphadenitis.

Bio-containment: BSL-2.

Diagnosis: cultivation (BA, $5 \% \mathrm{CO}_{2}$ ), intradermal inoculation of laboratory animals (mouse in the tail, rabbit in the auricle).

Treatment: penicillin + streptomycin, tetracycline, chloramphenicol, sulphonamides, locally potassium iodide; surgery.

Geographical distribution: worldwide, sporadic.

\subsubsection{Family Erysipelotrichaceae [Order "Incertae sedis", Phyllum Firmicutes]}

\section{(*) Erysipelothrix rhusiopathiae}

Non-motile pleomorphic non-sporulating Gram-positive rods $0.8-2.5 \times 0.1-0.3 \mu \mathrm{m}$.

Source of infection (natural host range): pig (E. rhusiopathiae var. suis) and other domestic and wild mammals (especially rodents $-E$. rhusiopathiae var. murisepticum), also domestic birds (turkey, chickens), fish, cuttlefish, molluscs, crabs, lobsters; water.

Animal disease: swine erysipelas with acute or chronic course (rash, septicaemia, endocarditis, arthritis), sometimes clinical disease in domestic and wild birds.

Transmission mode: contact (percutaneous at injury: occupational disease in butchers, veterinarians, fishers, pickers and shope assistants of seafood, farmers); exceptionally haematophagous arthropods (isolation of E. rhusiopathiae from ixodid ticks - experimentally confirmed mechanical (?) transmission by Ixodes persulcatus, mites, fleas and flies); considerable tenacity of the agent (in water and soil it survives at $5^{\circ} \mathrm{C}$ up to 970 days, in animal cadavers up to 9 months, it also resists higher concentrations of $\mathrm{NaCl})$.

Human disease: erysipeloid [vs. erysipel caused by Streptococcus pyogenes] characteristic erythema on hands, oedema of finger joints; infrequently a generalized (septic) form with polyarthritis and endocarditis (in that case the fatality rate is high).

Bio-containment: BSL-2.

Diagnosis: symptoms and anamnesis (haemo)cultivation (BA with glucose, 5-10\% $\mathrm{CO}_{2}$ ), subcutaneous inoculation of mouse.

Treatment: penicillin, erythromycin (tetracyclines). 
Prevention: vaccination of animals.

Geographical distribution: worldwide.

\subsubsection{Family Listeriaceae [Order Bacillales, Class Bacilli]}

\section{(*) Listeria monocytogenes (L. ivanovii)}

Short, motile ("tumbling" at lower temperatures of $20-25^{\circ} \mathrm{C}$ ), non-sporulating aerobic Gram-positive rods $0.5-2 \times 0.4-0.6 \mu \mathrm{m}$. A total of 13 serotypes have been described, in humans occur most frequently the serovars $1 / 2 \mathrm{a}, 1 / 2 \mathrm{~b}$, and $4 \mathrm{~b}$.

Source of infection (natural host range): (1) domestic and wild mammals (sheep, goat, cattle, pig, rodents), birds (corvids, gulls, fowl), and man (up to $5 \%$ of the human population are asymptomatic carriers); (2) soil, water, fodder, silage, household rubbish (L. monocytogenes grows even in fridge at $\left.4^{\circ} \mathrm{C}\right)$.

Animal disease: often inapparent infection, sometimes encephalitis, abortions (especially in sheep), and fatal septicaemia in young fowl, lambs and calves.

Transmission mode: nearly exclusively alimentary ("food-borne" infection: soft maturing cheese sorts, raw milk, smoked tongue, ham, paté, raw cabbage, salads, sea fish and other seafood), less often contact (farmers, fishers), aerogenic, via conjunctivae, and transplacentary. The agent's tenacity is remarkable: it survives at a lower temperature 1 year or more, and tolerates, e.g., $10 \% \mathrm{NaCl}$. L. monocytogenes also survives in macrophages, and its virulence is activated by contact with gastric fluid of the host.

Human disease: listeriosis - often inapparent infection. Incubation period of listeriosis is very long, on the average one month but sometimes up to 70 days. The symptoms of listeriosis are fever, headaches, chills, pharyngitis, nausea, mononucleosis, purulent meningitis (in up to a quarter of cases), encephalitis, endocarditis, granulomas and abscesses in liver, subcutis and other organs, septicaemia (bacteraemia) in half of the cases; fatality rate is up to 20-30\% (mortality usually occurs in old persons, at immunosuppression or at a large infectious dose). Characteristically, there is no enteritis. A dangerous disease is the listeriosis in pregnant women usually leading to miscarriage or pre-term delivery of babies associated with septicaemia, severe damage to CNS and visceral organs; fatality rate is high, 30-80\%. L. monocytogenes has namely a considerable tropism for the CNS, placenta and uterus of mammals. Extensive epidemics of listeriosis after consumption of contaminated food (cheese etc.) occurred in USA, Switzerland, France, Denmark and Germany (serotype 4b, a few hundreds of persons) in the years 1985-1990. A total of 1,566 persons (292 of them hospitalized) were infected with the serotype 4 b of L. monocytogenes in northern Italy in 1999; the source of infection was a maize salad with tuna fish. An increased incidence of listeriosis was also observed in UK and other European countries in 2001 and 2003. In the 
European Union the incidence of listeriosis has been growing moderately since 2004: from 1,264 total cases, to 1,427 cases in 2005, 1,583 in 2006, and 1,554 in 2007; it corresponds to the incidence rate of 0.3 per 100,000 population; the mortality rate remains high, about $20 \%$. L. ivanovii is an enteric opportunistic pathogen, causing occasionally gastroenteritis and bacteraemia in man; the isolates are indistinguishable from ruminant strains.

Bio-containment: BSL-2.

Diagnosis: cultivation of the blood, CSF, placenta samples (MPA, BA; optimally after a semi-selective replication in enrichment broth at $4^{\circ} \mathrm{C}$ and subsequently at $37^{\circ} \mathrm{C}$ ), CAMP test with Rhodococcus equi or Staphylococcus aureus, inoculation of mouse (treated with cortisone); serology less useful (CFT), PCR.

Treatment: ampicillin, azithromycin, gentamicin, large doses of penicillin plus kanamycin, tetracycline, chloramphenicol.

Geographical distribution: worldwide (less often in tropics), ubiquitous. Within Europe, listeriosis occurs more frequently in countries that produce a lot of maturing cheese (e.g. France, Switzerland, Germany, the Netherlands, Italy).

\subsubsection{Family Bacillaceae [Order Bacillales]}

\section{$\left(^{*}\right)$ Bacillus anthracis}

Greek " $\alpha \nu \theta \rho \alpha \xi$ " (anthrax) means "glowing coal" (cutaneous form of the disease is characterized by a black skin lesion). An aerobic, non-motile Gram-positive rod 4$8 \times 1-1.5 \mu \mathrm{m}$ with a mucous polyglutamate capsule and a spore located in the cell centrally, as observed already by Robert Koch in 1876 who cultivated the anthrax bacillus first. Virulence is determined by a plasmid.

Source of infection (natural host range): domestic and wild ruminants (especially cattle, sheep and goat), equids; soil (on pastures contaminated with the agent or its spores mainly in alluvial areas of rivers, or in deltas), animal products (manure, skins, fur, wool).

Animal disease: anthrax (herbivores, much less pigs and carnivores) - a peracute septic illness with a sudden death. For instance, ten thousands of livestock died in Zimbabwe in 1979-1982; about 70 cattle, horses and pigs died in Romanian Danube delta in 2000. An epizootic of bisons in the USA, 2007.

Transmission mode: contact (percutaneous, e.g. at sloughter of an infected animal), aerogenic, inoculative by biting insects or in heroin addicts (many recent cases in the UK), alimentary (water); the spores of $B$. anthracis are very resistant (in the soil they remain viable for tens of years: enzootic foci in inundated pasture areas).

Human disease: anthrax - according to the transmission mode there are the forms cutaneous (pustula maligna - a necrotic black scare); pulmonary; 
abdominal; and septic. The number of anthrax cases in the world varies between 5 and 100,000 yearly (WHO), with a considerable mortality (25$100 \%$ ) especially in non-cutaneous forms of the disease with septicaemia (the fatality rate in cutaneous forms is 5-20\%). Epidemics: e.g., Zimbabwe c. 10,000 cases in 1979-1985 (the source: infected cattle); 64 persons died in Yekaterinburg (former Sverdlovsk) after an unintentional release of B. anthracis spores in 1979 from a plant producing bacteriological weapons; 2000 Volgograd: 25 persons were infected after consuming infected meat; 2000 Romanian Danube delta: 2 persons died. In 2001, anthrax spores were used as a bacteriological weapon in letters in USA: 22 persons were infected, and 5 of them died (11 cases were the inhalational clinical form, and 11 were the cutaneous form). Occupational disease: the wool, skin and fur proceeding workers (carpets etc.), veterinarians, butchers, farmers, diggers in burial ground with the anthrax contaminated bodies.

Bio-containment: BSL-2/3. Potential bioterrorist agent (used, e.g., in USA).

Diagnosis: symptoms (in cutaneous form), blood smear (Gram, IF) with detection of capsules (Giemsa or polychromic methylene blue stain, or phase contrast); cultivation (MPA, BA: colonies have a characteristic shape caput Medusae, without haemolysis; selective media such as PLET agar inhibiting Bacillus cereus); inoculation of laboratory mouse (or guinea pig, rabbit); Ascoli's precipitation reaction (overlaying of anti-anthrax precipitation serum with the filtered extract of the tested organ); serology (IFA), PCR.

Treatment: large doses of penicillin, amoxicilin, ampicillin, penicillin combined with streptomycin; tetracycline, chloramphenicol, erythromycin.

Prevention: vaccine (immunization with a toxoid; for humans and animals, a non-virulent strain; developed Louis Pasteur in 1880, who cultivated the strain for 6 weeks $/ 42-43^{\circ} \mathrm{C}$ ); (a vaccine licensed in USA - AVA/Biothrax is applied by skin scarification). In leather manufacture, inactivation of B. anthracis spores with peracetic acid (Persteril) is used for raw materials (skins).

Geographical distribution: worldwide, but sporadic (enzootic foci) - Asia (Siberia, Turkey, Iran, Iraq), Europe (Romania, southern Russia, Hungary), Africa, South America.

\subsubsection{Family Staphylococcaceae [Order Bacillales]}

\section{(**) Staphylococcus aureus, S. epidermidis, S. haemolyticus, S. intermedius and Other spp.}

Non-motile Gram-positive cocci of about $1 \mu \mathrm{m}$, in clusters, facultatively anaerobic and producing catalase. S. aureus and S. intermedius also produce plasmacoagulase, haemolysin, and ferment mannitol. 
Source of infection (natural host range): cattle, dog (S. intermedius), pig and horse (MRSA zoonotic strains) and other mammals; man. The MRSA strains associated with livestock are called LA-MRSA.

Animal disease: mastitis in cows (an economically very important illness), dermatitis, arthritis, abscesses, and abortions.

Transmission mode: contact, alimentary (milk and dairy products: e.g., 15,000 patients were infected after drinking infected milk in Japan in 2000; meat). Occasionally nosocomial infections. Some staphylococcal strains have a considerable tenacity.

Occupational risk: farm workers.

Human disease: staphylococcosis - dermatitis (3-11\% isolates are of animal origin, e.g. sequence type $S$. aureus ST 398 in the Americas; $S$. intermedius rarely - after dog bites) and other suppurative processes, abscesses, osteomyelitis, sinusitis, respiratory illness, endocarditis, arthritis. Staphylococcal enterotoxicosis with enterotoxigenic strains (gastroenteritis) with an incubation period as short as $2-6 \mathrm{~h}$; the incidence of this illness is generally increasing. Sometimes septic shock, especially with MRSA possessing so-called superantigen; many of these strains are zoonotic, originating from domestic animals. Staphylococci (including zoonotic) produce a number of exotoxins (hyaluronidase, coagulase, $\alpha$ - and $\beta$-haemolysin, enterotoxins, exfoliatins etc.) that together with other virulence factors (peptidoglycan, protein A etc.) very strongly affect pathogenesis of the infection.

Bio-containment: BSL-2.

Diagnosis: cultivation (e.g. on BA, or selective media with mannitol and $10 \%$ $\mathrm{NaCl}$ ), coagulase test, phagotypization, molecular typization; serological detection of toxin.

Treatment: antibiotics (optimally after the susceptibility testing) - oxacilin, methicillin (but some strains are MRSA - methicillin resistant $S$. aureus), chloramphenicol, tetracycline, ampicillin, erythromycin; junction of abscesses. There is no specific therapy for staphylococcal enterotoxicosis.

Geographical distribution: worldwide.

\subsubsection{Family Streptococcaceae [Order Lactobacillales, Class Bacilli]}

\section{Streptococcus suis, S. equi ssp. zooepidemicus}

Pyogenic streptococci of the $\alpha$-haemolytic group D (S. suis), and $\beta$-haemolytic group C (S. equi ssp. zooepidemicus). Non-motile cocci $1 \mu \mathrm{m}$, arranged in chains, do not produce catalase. 
Source of infection (natural host range): domestic and wild swine (S. suis, reservoir, the agent occurs mainly on tonsils and in vagina), horse ( $S$. equi ssp. zooepidemicus).

Animal disease: purulent inflammation of upper respiratory tract, with lymphadenitis (in horse, S. equi ssp. zooepidemicus), vaginitis and uterus inflammation (mare, S. equi ssp. zooepidemicus), less often mastitis in cattle and arthritis in lambs (S. equi ssp. zooepidemicus); bronchopneumonia, endocarditis, polyarthritis, septicaemia and meningitis in swine with fatality rate up to $50 \%$ (S. suis, mainly serotype 2 ).

Transmission mode: alimentary (raw milk and cheese, undercooked pork S. suis) or aerogenic (S. equi ssp. zooepidemicus), contact (percutaneous skin injury).

Occupational risk: farmers, swine breeders, butchers, slaughterhouse workers, cooks (S. suis).

Human disease: streptococcosis (purulent meningitis, septicaemia); in S. equi ssp. zooepidemicus pneumonia, septicaemia, endocarditis, nephritis; arthritis, meningitis, osteomyelitis; in $S$. suis fever, meningitis, deafness, (poly)arthritis, uveitis, diplopia, endocarditis, pneumonia, peritonitis, septicaemia, ataxia, toxic shock syndrome, with the fatality rate about 8-20\%. The first case of human infection with S. suis was described in Denmark in 1968, later a few cases in other European countries (the Netherlands, England, Czechland, Slovakia, Sweden, Belgium, Germany, France, Hungary, Austria, Croatia, Italy, Spain, Greece), Canada, Hawaii, China (1998: 25 cases including 14 deaths), etc.; up to 2005, the total number of documented cases in the world was 410 . However, a major outbreak of $S$. suis infections occurred in Sechuan (China) with 218 severe cases (in farmers) in 2005, mostly as the toxic shock syndrome and fatality rate of 19\%. Another outbreak occurred in Vietnam, 2007, involved 45 cases (at least two patients died).

Bio-containment: BSL-2.

Diagnosis: anamnesis; cultivation (BA) of throat swabs, CSF and blood samples; biochemical tests (API 20 Strep System); serotypisation; MLST. S. suis might be misidentified as a member of the viridans group streptococci.

Treatment: penicillin.

Geographical distribution: worldwide, S. suis is more common in southeast Asia, and China sporadic in mild climatic zone (UK, the Netherlands, Germany, Czechland, Slovakia, Croatia, Greece, New Zealand, USA, Canada).

\section{Streptococcus iniae}

Non-motile streptococci $1 \mu \mathrm{m}$ in diametre, do not produce catalase. Virulence factors similar to those found in group A streptococci (S. pyogenes). 
Source of infection: fish.

Animal disease: fish pathogen (sporadic infections in tilapia, yellowtail, rainbow trout, and coho salmon as well as disease outbreaks with high mortality rates in aquaculture farms) - skin lesions, necrotizing myositis, meningoencephalitis, panophthalmitis; sometimes asymptomatical course.

Transmission mode: percutaneous (throw soft tissue injuries that may occur during the preparation of fresh fish from wet markets).

Human disease: streptococcosis, usually results in bacteraemic cellulitis of the hand, accompanied with one or more of these symptoms: endocarditis, meningitis, arthritis, sepsis, pneumonia, osteomyelitis, and toxic shock. First human case of $S$. iniae infection was reported in 1996, and since 2000, hundreds of cases have been reported from southern Asia and Australia. Most of infections occur in elderly people who commonly have underlying conditions such as diabetes mellitus, chronic rheumatic heart disease, cirrhosis, etc.

Bio-containment: BSL-2.

Diagnosis: anamnesis, cultivation (BA), PFGE, MLST (multi-locus sequence typing).

Treatment: penicillin, ampicilin, cloxacillin, cefazolin, and/or gentamycin, doxycycline, and trimethoprim/sulphamethoxazole.

Geographical distribution: probably worldwide (global distribution among fish population), largely North America, Israel, and Asia (Japan, Taiwan, China).

\section{Enterococcus faecalis (VRE, HLGRE)}

Enterococci are gut commensals in humans and other endothermic vertebrates. But the emerging vancomycin-resistant (VRE) and high-level gentamicin-resistant (HLGRE) enterococci (multilocus sequence type 16) can cause serious diseases, e.g. endocarditis.

Source of infection: some HLGRE and VRE isolates from pigs are identical with those from humans sufferring from endocarditis.

Animal disease: not observed.

Transmission mode: alimentary, by contact. Nosocomial infections.

Human disease: endocarditis, sometimes severe.

Bio-containment: BSL-2.

Diagnosis: by cultivation (BA, Slanetz-Bartley or kanamycin-aesculin-azid medium) and antibiotic sensitivity testing.

Treatment: normally combinations of antibiotic such as one affecting cell wall of bacteria (ampicillin, penicillin, or vancomycin) plus gentamicin or vancomycin. But the HLGRE and VRE strains pose now great problems. The alternative use is linesolid, quinupristin or some fluoroquinolones.

Geographical distribution: USA, Europe (e.g., Denmark), but probably also many other geographic areas. 


\subsubsection{Family Clostridiaceae [Order Clostridiales, Class Clostridia]}

\section{${ }^{*}$ Clostridium tetani}

Anaerobic slim Gram-positive rod 4-8 $\times 0.4-0.8 \mu \mathrm{m}$ with a terminal spore of the plectridium type.

Source of infection: soil (especially manured); horse and other mammals.

Animal disease: tetanus (dogs, cats and birds are resistant).

Transmission mode: by contact (percutaneous - injury: e.g., farmers, gardeners); spores are very resistant, they survive (e.g. in soil $>10$ years, and in boiling water up to $3 \mathrm{~h}$ ).

Human disease: tetanus (lockjaw); classical neurotoxicosis (main toxin is tetanospasmin, its MLD for mouse is $0.0001 \mu \mathrm{g}$; another one is tetanolysin), with the fatality rate of 30-70\%; first clinical signs are the blockade (painful contraction) of maxillary muscles, trismus (sardonic grimace) and abdominal rigidity, later convulsions of back muscles (opisthotonus: bow-shaped deflection of body to the back, leading sometimes to fractures of vertebrae and muscles), respiratory and other muscles; no fever, consciousness is conserved.

Bio-containment: BSL-2/3.

Diagnosis: clinical symptoms (tonic spasms); microscopy and anaerobic cultivation of wounded tissue, subcutaneous inoculation of mice (combined with toxin neutralization test).

Treatment: surgical treatment of the wound, application of specific human (TEGA) or horse immunoglobulin (antitoxin, antitetanic serum) within $24 \mathrm{~h}$; pulmonary ventillation or tracheotomy, myorelaxants and sedatives; penicillin and metronidazole.

Prevention: immunization with anatoxin (toxoid).

Geographical distribution: worldwide (less frequent in cold areas).

\section{${ }^{*}$ Clostridium perfringens Type A, C. septicum, C. novyi etc.}

Anaerobic Gram-positive rods 4-6×0.6-1 $\mu \mathrm{m}$, non-motile $(C$. perfringens) or motile (C. septicum, C. novyi).

Source of infection: soil (contaminated with animal excrements).

Animal disease: gastritis in lambs (C. septicum); necrotic hepatitis in sheep (C. novyi).

Transmission mode: by contact (injury, trauma - car accidents and other harms; iatrogenic).

Human disease: gas gangrene - cellulititis, myositis, fasciitis, necrosis of tissue with gas production (main toxin is lecithinase - phospholipase C); fatality rate is $30-90 \%$. A total of 35 (out of 104 infected drug-users) died of sepsis 
after syringe application of contaminated drugs in Great Britain in 2000 (C. novyi, C. perfringens).

Bio-containment: BSL-2/3.

Diagnosis: clinical symptoms, microscopy and anaerobic cultivation of the tissue, detection of lecithinase.

Treatment: surgical treatment of wounds; antibiotics (penicillins) in high doses; hyperbaric box.

Geographical distribution: worldwide.

\section{(*) Clostridium difficile, $C$. perfringens}

Anaerobic Gram-positive rods. Highly virulent ribotypes O17, O27, and $\mathrm{O} 78$ of C. difficile emerged lately.

Source of infection: soil, water, digestive tract of vertebrates (pig, cattle, poultry); man. Ribotypes of many human isolates have been shown to be identical with bovine strains.

Animal disease: calf diarrhoea, infectious enterotoxemia of domestic animals.

Transmission mode: alimentary (food-borne: e.g., undercooked and longer weaned meat, salads); nosocomial - man to man transmission (about 5\% of adults act as carriers of $C$. difficile occurring in the gut).

Human disease: intestinal clostridiosis - infection or intoxication (toxin production in the gut - enterotoxins A and B) - pseudomembraneous colitis with diarrhoea, occasionally occurrence of megacolon with risk of gut perforation and subsequent peritonitis; gastroenteritis with diarrhoea and abdomen convulsions, and quite high lethality. The disease frequently occurs after long-term treatment with various antibiotics (so-called "post-antibiotic colitis"), when $C$. difficile replicates abundantly in the gut and produces toxins (the most virulent are the strains with type $\mathrm{V}$ toxin). C. difficile forms considerable portion of bacterial enteric infections in old peoples' homes, hospices and some clinics (a hospital ecological niche); the incidence rate of this disease, sometimes called CDAD ( $C$. difficile-associated disease) has increased tenfold recently - it is an emerging nosocomial infectious disease, spreading as anthroponosis especially in North America, Europe and Singapore. People older than 65 years are very susceptible to this illness.

Bio-containment: BSL-2.

Diagnosis: anaerobic cultivation of stool samples and foods (selective media with cefoxitin and cycloserine), detection of enterotoxin in the stool and blood specimens on tissue cultures or by ELISA.

Treatment: rehydration, vancomycin, metronidazole. However antibiotic treatment increases incidence of $C$. difficile infections in healthcare settings.

Geographical distribution: worldwide, with increasing incidence in countries associated with high hygienic standard (Canada, Great Britain, Belgium, Netherlands, France, Austria). 


\section{(*) Clostridium botulinum}

An anaerobic motile rod 4-6(10) $\times 1 \mu \mathrm{m}$, with a subterminal spore.

Source of infection (natural host range): pig, ruminants, horse, poultry; fish (type E); soil, vegetables, fruit, silage.

Animal disease: botulism (death caused by diaphragm paralysis) in mammals and birds (chicken, wild waterfowl).

Transmission mode: alimentary (gastric trypsin activates the toxin) - ham, sausages, home-made tins of meat, vegetable (green beans, peas, root vegetables) and fish origin, liver pate; wound botulism (drug users).

Human disease: botulism (alimentary intoxication of humans by the types A, $\mathrm{B}, \mathrm{E}, \mathrm{F}$ and $\mathrm{G}$, the most effective neurotoxins at all causing after an incubation period of $12 \mathrm{~h}$ to several days irreversible neuromotoric paralysis by blockade of acetylcholine release on synapses, with loss of motility, diplopia, strabism, paralysis of respiratory muscles); fatality rate $15-75 \%$. MLD of botulinum toxin ("sausage poison") for man is $0.0625 \mu \mathrm{g}$. Very dangerous is infant botulism with replication of clostridia and toxin production in the gut of babies (honey as an addition to baby food is the most frequent source of C. botulinum).

Bio-containment: BSL-2.

Diagnosis: detection of botulotoxin in serum, faeces, vomitus or food residues by toxin-neutralization test on mice (ELISA is $10-100 \times$ less sensitive); cultivation of the stool or of food residues is less important.

Treatment: stomach and intestine lavage; botulinum antitoxin poly- or monovalent.

Prevention: vaccination with toxoid (in occupations with risk, and animals).

Geographical distribution: worldwide.

\subsubsection{Family Mycobacteriaceae [Order Actinomycetales, Class Actinobacteria]}

\section{Mycobacterium bovis}

Non-motile, chromophobic by Gram (but de facto Gram-positive) acidoresistant irregular rods $3.0 \times 0.3 \mu \mathrm{m}$. Diaminopimelic and mycolic acids (waxes) are included in cell wall of mycobacteria. Genomically very closely related are M. microti, M. caprae, and M. pinnipedii, jointly belonging to the M. tuberculosis complex, and occasionally transmissible to humans.

Source of infection (natural host range): cattle and other domestic (occasionally pig, cat, camel) and free-living mammals (e.g., badger Meles meles is reservoir in England; wild boar Sus scrofa is reservoir in some countries of continental Europe, African buffalo Syncerus caffer, red lechwe Kobus leche, 
greater kudu Tragelaphus strepsiceros and warthog Phacochoerus africanus in Africa; American bison Bison bison and deers in North America; or common brushtail Trichosurus vulpecula in New Zealand). Source of infection in M. microti are small terrestrial mammals (voles).

Animal disease: tuberculosis of domestic (bovine tbc) and free-living ruminants (bison in Canada), pig, cat, dog - chronic disease with tubercles in the lungs and other tissues.

Transmission mode: aerogenic, alimentary (raw milk and milk products, undercooked meat); high tenacity of the agent.

Human disease: bovine tuberculosis (clinically indistinguishable from the human tuberculosis) - pulmonary, more often extrapulmonary (urogenital, gastrointestinal, bone, meningeal or skin), slow progressive disease accompanied by fever and cervical lymphadenopathy (scrophula); incubation from 1 month up to several years. It could lead in lethal disease without sufficient therapy. Immunocompromised persons seem to be the most susceptible (e.g., with HIV infection). Zoonotic tuberculosis constitutes in different regions $3-10 \%$ of overall tuberculosis cases in humans. Disease caused by $M$. microti occurs only rarely in human (a total of 11 cases have been described), usually in patients with HIV, but also two immunocompetent patients suffered from pulmonary tuberculosis due to $M$. microti in Germany in 1999. Vaccine strain (BCG) occasionally causes postvaccination infections in immunosuppressed patients. [M. tuberculosis sensu stricto or "BK", Koch's bacillus, is causative agent of human tuberculosis (an anthroponosis)].

Bio-containment: BSL-3.

Diagnosis: X-ray computed tomography, microscopy (Ziehl-Neelsen stains of acidoresistant microbes: hot karbolfuchsin, decolouration with alcohol-3\% $\mathrm{HCl}$, another staining with malachite green or methylene blue) and difficult, up to 8-week cultivation of sputum (after treatment of the sputum samples with $4 \% \mathrm{NaOH}$ or $\mathrm{HCl}$ and subsequent neutralization), CSF, urine or pus on Löwenstein-Jensen egg medium, Šula's or Ogawa media with glutamate; also tuberculin skin test (allergic response). Multiplex PCR. Molecular identification by means of RFLP typing; inserted sequence IS6110 is characteristic for this complex.

Treatment: isoniazid + rifampicin, pyrazinamide, ethambutol; formerly streptomycin and other tuberculostatics (p-aminosalicylic acid, cycloserine, ethionamide etc.).

Prevention: attenuated vaccine $M$. bovis BCG (bacillus Calmette-Guérin: effectiveness not optimal, about $80 \%$ ); eradication programs in domestic animals. Attenuated vaccine from strain VO166 M. microti has been formerly used in Czech Republic and Great Britain. Vaccine strain (BCG) occasionally causes post-vaccination infections in immunosuppressed patients.

Geographical distribution: worldwide (but eradicated in many countries). 


\section{Mycobacterium avium Complex}

Rods $1-2 \times 0.5 \mu \mathrm{m}$. Non-pigmented mycobacteria with subspecies $M$. a. avium (causative agent of avian tuberculosis), M. a. silvaticum (the wood pigeon strains, pathogenic for wild and domestic birds), M. a. paratuberculosis (99.9\% similarity of $16 \mathrm{~S}$ rRNA with $M$. a. avium, causative agent of paratuberculosis in ruminants and other mammals), and probably non-zoonotic $M$. a. hominissuis (causes avian mycobacteriosis in pigs, cattle and other animals including human) and M. a. intracellulare (the latter is sometimes recognized as distinct species because DNA-DNA hybridization studies demonstrate that $M$. avium and $M$. intracellulare strains only share approximately 50\% similarity). A new, sapronotic mycobacterium species is *M. chimaera, closely related to M. intracellulare.

Source of infection (natural host range): poultry (M. a. avium) and other birds (rook, pheasant, sparrow, woodpigeon, feral pigeon - M. a. avium, M. a. silvaticum), pig, rarely cattle in $M$. a. avium; cattle, sheep, goat in $M$. a. paratuberculosis (also wild mammals like red deer, roe deer, mouflon, chamois, wild boar, rabbit, etc.).

Animal disease: avian tuberculosis; lymphadenitis (M. a. avium), mesenteritis (M. a. intracellulare) in pigs; Johne's disease (M. a. paratuberculosis) paratuberculosis, a veterinary important chronic hypertrophic enterititis in cattle with diarrhoea and anorexia, leading to death.

Transmission mode: alimentary (milk, less often meat), aerogenic; high tenacity of the agent in environment (M. a. avium is viable in water for about 113-417 days, in humid excrements up to 400 days; M. a. paratuberculosis usually survives in excrements of cattle and also in the soil up to 1 year). M. chimaera is present in water-cooling towers and circuits, the transmission is aerogenic.

Human disease: avian tuberculosis - pulmonary lesions, lymphadenitis, gastrointestinal lesions, after dissemination even fatal; incidence is increasing (frequent in immunocompromised persons with AIDS, etc.). Lesions are typically without necroses, calcification and fibroses. M. a. paratuberculosis: paratuberculosis, perhaps also Crohn's disease (multifactorial chronic granulomatous enteritis or ulcerative colitis with relapses). Infectious aetiology of Crohn's disease has not been proven reliably until present, however an association with paratuberculosis exists: presence of antibodies and enhanced detection of $M$. a. paratuberculosis in the patients. The prevailing opinion is that Crohn's disease has a multifactorial aetiology.

Bio-containment: BSL-2.

Diagnosis: as in the previous species; microscopy and histopathology; cultivation seems difficult, PCR detection, serology (CFT) in M. a. paratuberculosis. Molecular differentiation within the M. avium complex is based on DNA fingerprinting by RFLP typing-inserted DNA sequences IS900 (present only in M. a. paratuberculosis), IS901/902 (only present in M. a. avium) and IS1245 (in M. a. avium and M. a. hominissuis). 
Treatment: difficult (number of strains are resistant) and long-term (at least 24 months), relapses of the diseases occur quite often. Therapy with isoniazid + rifampicin, pyrazinamide, ethambutol.

Geographical distribution: M. a. avium worldwide. M. a. paratuberculosis in New Zealand, Australia, Europe (Great Britain, France, Netherlands, Denmark, Germany, Belgium, Italy, Austria, Switzerland, Czechland, etc.), USA. M. chimaera has been detected in Germany and Oman.

\section{*Mycobacterium kansasii}

First described as "yellow bacilli"; "atypical", slow-growing "non-tuberculous" saprophytic mycobacteria, however potentially pathogenic for human. Optimum growth temperature is $30-33^{\circ} \mathrm{C}$, but it is capable of growth at $40-45^{\circ} \mathrm{C}$.

Source of infection: industrial, technical, and tap water (forming biofilms in water piping, shower heads, aquaria), soil and dust; amoebae, cockroaches, and some mammals (goat, monkey, dog, cattle).

Transmission mode: aerogenic, alimentary; iatrogenic.

Human disease: mycobacteriosis - chronic pulmonary disease (risk factor for acquiring infection: occupational exposure in coal miners; e.g., in Ostrava (Czechland), a total of 1,034 patients were recorded in 1968-1999), more often in immunocompromised persons (AIDS, chronic bronchitis, history of tuberculosis, pneumoconiosis, or people with lung parenchyma damage). Extrapulmonary forms of the disease involve skin lesions, cervical lymphadenitis, and uterine infections. A case of fatal septicaemia was also demonstrated. During an epidemiological survey in Spain in 2000-2003, a total of 598 cases were identified, with distinct clinical (mainly pulmonary) manifestation in $74 \%$ of them.

Bio-containment: BSL-2.

Diagnosis: see other mycobacteria; the optimum temperature for its in vitro isolation ranges between 30 and $33^{\circ} \mathrm{C}$.

Treatment: clarithromycin + ciprofloxacin.

Geographical distribution: worldwide.

\section{*Mycobacterium xenopi}

"Atypical", mostly slow-growing "non-tuberculous" saprophytic mycobacteria, however potentially pathogenic for human; maximum growth temperature is between 42 and $45^{\circ}$ C. M. xenopi was found to be more heat-resistant than Legionella pneumophila.

Source of infection: potable warm water systems (households, hospitals, dental units), animals (domestic and wild pig, cat, cattle), soil (?).

Transmission mode: aerogenic, alimentary. 
Human disease: mycobacteriosis - chronic pulmonary and extrapulmonary disease, especially in immunocompromised persons (AIDS). Usually sporadic disease, but outbreaks of pulmonary mycobacteriosis due to M. xenopi may rarely occur.

Bio-containment: BSL-2.

Diagnosis: see other mycobacteria.

Treatment: clarithromycin + ciprofloxacin.

Geographical distribution: worldwide.

\section{*M. marinum, M. haemophilum}

"Atypical" photochromogenic (M. haemophilum is not photochromogenic), mostly slow-growing "non-tuberculous" saprophytic mycobacteria, however potentially pathogenic for human. M. haemophilum demands special growth conditions (ferric ions).

Source of infection: water (aquaria - water, plants, sediment, biofilm, aquatic invertebrates, swimming pools, coal mine water, surface water, sewage, drinking water systems, soil, peat); fish (M. marinum - this species is also ichtyopathogenic); fish (zebrafish), insects, reptiles in M. haemophilum.

Transmission mode: by contact - skin injury.

Human disease: mycobacteriosis - skin disease characterized by rash, tubercles and raddish scabs 2 weeks after infection, then granulomas, nodules and ulceration evolve (M. marinum: "swimming pool granuloma" or "fish tank granuloma"); often in fish breeders and aquarists - usually after injury also M. haemophilum. Typical location of skin lesions are elbows, knees, lower legs, hands and fingers in amateur fishermen and aquarists; cervical lymphadenitis was recorded mostly in Dutch children (M. haemophilum).

Bio-containment: BSL-2.

Diagnosis and therapy: biopsy of damaged tissue for laboratory testing (culture, histology); optimum growth temperature for in vitro isolation ranges between 30 and $33^{\circ} \mathrm{C}$; for other methods see previous species.

Geographical distribution: worldwide.

\section{*Mycobacterium abscessus, M. chelonae, M. fortuitum, M. phocaicum}

"Atypical", mostly fast-growing "non-tuberculous" saprophytic mycobacteria, however potentially pathogenic for human; optimum temperature growth usually at $28-30^{\circ} \mathrm{C}$.

Source of infection: drinking water (hospital water supplies, showers, cooling towers, consumption of ice, ground water (M. chelonae, M. fortuitum), treated surface water, biofilms (bronchoscopes, dialysers)).

Transmission mode: aerogenic, alimentary, iatrogenic. Some of these mycobacteria can survive in amoebae. 
Human disease: mycobacteriosis - chronic pneumonia, abscesses; most infections that have been reported are nosocomial - contaminated instruments and fluids (in conjuction with cardiac bypass, dialysis, plastic surgery, postinjection abscesses), or in immunocompromised patients (AIDS etc.).

Bio-containment: BSL-2.

Diagnosis: see other mycobacteria; patterns of large restriction fragments by PFGE, patterns of mycolic acids separated by HPLC, sequencing of $16 \mathrm{~S}$ rRNA genes (gold standard).

Treatment: klaritromycin + ciprofloxacin, sulphonamides, cefoxitin, amikacin, ciprofloxacin, imipenem in $M$. fortuitum, tetracycline.

Geographical distribution: worldwide.

\section{*Mycobacterium ulcerans}

This microorganism evolved by means of ecological specialization from M. marinum (reduction of genome caused by DNA deletions from 6.6 to $5.8 \mathrm{Mb}$ ), as found by molecular analyses. Ten genotypes associated with geographical origin have been recognised until today. It does not grow at temperatures above $32^{\circ} \mathrm{C}$.

Source of infection: aquatic environment (stagnant water in swamps, less in rivers): aquatic plants (Echinochloa pyramidalis), insects and snails, small fish and other vertebrates (koala, possum and captive alpaca in Australia).

Transmission mode: by contact (injury) and inoculative (mechanically by aquatic insects and mosquitoes - during one Autralian outbreak in 2004 several strains of $M$. ulcerans were detected in mosquitoes in the endemic area).

Human disease: "Buruli ulcer" (Bairnsdale or Searls's ulcer in Australia, Kumusi ulcer in Papua New Guinea) - a chronic necrotizing infection of the skin, deep dermal and subcutaneous tissue, and bone. The clinical forms are either localized (nodules, minor ulcer, major ulcer) or disseminated (plaques, oedematous form, metastatic disease-may enter lymphatic system or bloodstream). Incidence in endemic areas is high; for instance in Benin, 2,598 new and recurrent cases were reported and treated in the years 2003 2005. Annual number of new cases is estimated in Africa as at least 4,000. Higher rates of infection with $M$. ulcerans are usually documented after flooding.

Bio-containment: BSL-2.

Diagnosis: see other mycobacteria.

Treatment: rifampicin, moxifloxacin, amikacin; in severe cases surgical excision of large ulcers, even amputations, are necessary.

Geographical distribution: tropics and subtropics - Africa (Angola, Benin, Burkina Faso, Cameroon, Democratic Republic of Congo, Gabon, Ghana, Guinea, Ivory Coast, Liberia, Nigeria, Sierra Leone, Sudan, Togo, Uganda), less often in (sub)tropical areas of Asia, Australia and Papua New Guinea, and Central and South America. 


\section{(*) Mycobacterium leprae}

This microbe has lost at least 2,000 genes after adaptation to human host (drastic reduction of genome, whose complete sequence of nucleotides has been described recently).

Source of infection: human; water (peatbogs and bryophytes growing in the surroundings of waterfalls and on fiord slopes with south exposition in Norway, Portugal, southern USA etc.), open water bodies (bathing) and contamination of clothes by washing in streams in endemic regions; systemic leprosy occurs occasionally in feral ninebanded armadillos.

Transmission mode: airborne - human to human transmission (thus anthroponosis); according to J. Kazda a L.W. Irgens transmission from environment either by ingestion of infected water (conducted by wood pipe-lines downhills in Norway) or by direct contact (scratches, from mother to child) is also possible.

Human disease: leprosy (Hansen's disease) - a chronic infection characteristic by skin, $\overline{\text { nervous }}$ or even general manifestations, without appropriate treatment leading in mutilations.

Bio-containment: BSL-2.

Treatment: long-term (6-12 months), with a combination of rifampicin, Dapsone, and clofazimine.

Diagnosis: microscopy of skin scrapings (staining according to Ziehl-Neelsen); it does not grow in vitro, while replicating in armadillo Didelphis and "nude" laboratory mice.

Geographical distribution: formerly worldwide, very limited at present.

\subsubsection{Family Corynebacteriaceae [Order Actinomycetales]}

\section{Corynebacterium ulcerans, C. pseudotuberculosis}

Short irregular Gram-positive rods $1-3 \times 0.4 \mu \mathrm{m}$, aerobic and non-motile.

Source of infection (natural host range): cattle, sheep, horse, dog, cat, pig and monkey (only C. ulcerans), occasionally other mammals; human.

Animal disease: pneumonia, abscesses and lymphadenitis.

Transmission mode: alimentary (raw milk), percutaneous (biting by monkey), by contact. Occupational risk in farmers and vets.

Human disease: pharyngitis (C. ulcerans) similar to diphtheria, wound infections (C. pseudotuberculosis) and regional lymphadenitis, pneumonia; a rare disease.

Bio-containment: BSL-2.

Diagnosis: cultivation of swabs (resp. smears) originated from wounds, milk (BA, selective agar with tellurate, Loeffler medium slants, 
Lowenstein-Jensen agar, some other media for isolation of mycobacteria);

inoculation of guinea pigs; detection of toxin.

Treatment: erythromycin, antitoxin.

Prevention: vaccination (diphtheric toxoid).

Geographical distribution: Great Britain, but obviously worldwide.

\subsubsection{Family Actinomycetaceae [Order Actinomycetales]}

\section{Arcanobacterium pyogenes}

Syn. Corynebacterium pyogenes. Slim Gram-positive rods fragmenting gradually into small coccobacilli, aerobic and non-motile.

Source of infection (natural host range): cattle, sheep, goat, pig, horse and other mammals; human.

Animal disease: abscesses, polyarthritis, lymphadenitis.

Transmission mode: by contact (via biting).

Human disease: purulent wound infections after biting and painful regional lymphadenitis, arthritis, sometimes sepsis; a quite rare disease.

Bio-containment: BSL-2.

Diagnosis: cultivation of scumming from wounds (BA, selective agar with tellurate); inoculation of guinea pig; detection of toxin.

Treatment: erythromycin.

Geographical distribution: probably worldwide.

\subsubsection{Family Nocardiaceae [Order Actinomycetales]}

\section{${ }^{*}$ Rhodococcus equi}

Synonym Corynebacterium equi. Pleomorphic coryneform Gram-positive, partially acidoresistant, aerobic cocci to rods (coccobacilli), forming short filaments with symptoms of branching.

Source of infection: soil enriched with faeces of domestic mammals ( $R$. equi is a commensal of their gastrointestinal tract), in which is the rhodococcus able to replicate.

Animal disease: acute or chronic bronchopneumonia with lung abscesses (sometimes lethal) of foals; lymphadenopathy in pigs.

Transmission mode: aerogenic, less often alimentary, contact with horses.

Human disease: rhodococcosis - fever, nausea, vomiting, diarrhoea, dizziness, granulomatous bronchopneumonia (opportunistic infection in immunocompromised patients), often lethal (fatality rate about 50\%); sometimes lymphadenitis and abscesses in the CNS, liver, kidneys and other organs, skin lesions in immunocompetent persons (mainly childrens) after injury may 
also occur. In patients with AIDS, the disease is often chronic and recurrent, because R. equi can survive intracellularly in macrophages. Incidence is quite low, about 200 cases have been described since the disease description in 1967.

Bio-containment: BSL-2.

Diagnosis: cultivation of blood, sputum, bronchial and tissue samples (BA, Czapek-Dox agar; pink colonies) and detection of "equi" factor (CAMP test: synergistic haemolysis against Staphylococcus aureus and other bacteria: partial haemolysis of S.a. turns into complete in proximity of R.e. colonies due to the effect of rhodococcal cholesteroloxidase); histopathology, acidoresistant staining (partially acidoresistant).

Treatment: long-term (2-9 months or even lifelong treatment) - rifampicin, vancomycin, erythromycin, gentamicin, imipenem, carbapenem, meropenem (often in combination of two drugs, e.g. sulbactam + cefoperazone; confirmation of in vitro sensitivity is needed).

Geographical distribution: probably worldwide.

\section{${ }^{*}$ Nocardia asteroides, $N$. brasiliensis}

Aerobic filaments of mycelial character, fragmenting into pleomorphic Grampositive rods to cocci, forming also aerial mycelium on solid substrates.

Source of infection: soil, plant remnants.

Animal disease: nocardiosis - infection of subcutis and lymph nodes (dog, cat), sporadically mastitis and pneumonia in cattle.

Transmission mode: aerogenic, percutaneous.

Human disease: nocardiosis pulmonary, cutaneous (actinomycetoma), visceral, even CNS affection (by dissemination from lungs, about $30 \%$ of cases); fatality rate $80 \%$ in CNS forms.

Bio-containment: BSL-2.

Diagnosis: microscopy of sputum, pus or CSF (Gram and acidophilic staining), cultivation (BA etc.); X-ray computed tomography.

Treatment: cotrimoxazole, sulphonamides in long-term usage; some strains sensitive to ampicilin, amikacin, cephalosporines and other antibiotics; surgical intervention (abscesses).

Geographical distribution: worldwide, however sporadic; $N$. brasiliensis occurs more frequently in (sub)tropics.

\subsubsection{Families Thermomonosporaceae, Streptomycetaceae [Order Actinomycetales]}

\section{*Actinomadura madurae, A. pelletieri, Streptomyces somaliensis}

Source of infection: soil, vegetation.

Animal disease: (actino)mycetoma. 
Transmission mode: percutaneous (injury - thorns etc.).

Human disease: actinomycetoma predominantly on lower extremities. It is distinguishable from (eu)mycetoma caused by fungi.

Bio-containment: BSL-2.

Diagnosis: microscopy of pus or biopsy (Gram and acidophilic staining), cultivation, X-ray examination.

Treatment: cotrimoxazole, streptomycin, amikacin; surgical intervention.

Geographical distribution: America, Africa, Arabic Peninsula - semiarid habitats in areas with dry and warm climate.

\subsubsection{Family Dermatophilaceae [Order Actinomycetales]}

\section{Dermatophilus congolensis}

Facultatively anaerobic microbe with heavily septate hyphae, dividing both transversally and longitudinaly, forming characteristic packages of Gram-positive coccoid cells; motile cells (spores) are also recognized, $0.3-0.5 \mu \mathrm{m}$.

Source of infection (natural host range): cattle, sheep, horse, mammals in ZOO, less often dog and cat (soil source of infection is disputable).

Animal disease: dermatophilosis (cutaneous streptothrichosis, sometimes falsely regarded as mycotic dermatitis) - pustules and papules, occasionally lethal (mainly in tropics). Symptoms increase in severity with infestation of the affected animal by ticks of the genus Amblyomma.

Transmission mode: percutaneous (by direct contact); possibly also by arthropods (ticks - Amblyomma variegatum, diptera - mechanically).

Human disease: dermatophilosis - acute to chronic pustules and ulcers on hands.

Bio-containment: BSL-2.

Diagnosis: microscopy of epidermal scales (Giemsa staining), histology, cultivation (without antibiotics - BHI, BA, while SGA not).

Treatment: streptomycin, penicillin.

Geographical distribution: areas with humid climate in Africa, Australia, USA and Europe (Scotland, the Netherlands, Bulgaria).

\subsection{Fungi}

In this chapter filamentous (with mycelium) and yeastlike eukaryotic microorganisms are dealt with. The taxonomic nomenclature of fungi differentiates two forms according to their life cycle: the anamorph is the asexual phase with asexual spores (conidia), or sterile; the teleomorph is the sexual phase with fruiting bodies and sexual spores (ascospores, basidiospores or zygospores). The complex of all developmental forms of the fungus (i.e., the teleomorph including all its anamorphs) is regarded as a holomorph, the binomical determination of which is identical with a teleomorph. 
Fungi (micromycetes) that are pathogenic for vertebrates cause two basic clinical syndromes of mycoses: (1) superficial diseases with lesions on the skin and its adnexes (nails, hairs etc.), and (2) visceral (deep, organ, systemic) diseases. Among the most common agents causing skin mycoses belong dermatophytes of the teleomorph genus Arthroderma (with the anamorphs Trichophyton, Epidermophyton and Microsporum); these dermatomycoses are differentiated according to the source of infection being antropophilic (corresponding to the adjective "anthroponotic" used throughout this book), zoophilic (corresponding to the adjective "zoonotic"), and geophilic (corresponding to the adjective "sapronotic"). Noteworthy, many agents causing visceral mycoses are dimorphic: their saprophytic phase is filamentous, but the parasitic phase appearing in tissues of warm-blooded vertebrates is morphologically entirely different - most often yeast-like, or spheruliform.

Among the first signs indicating that a patient could be suffering from a visceral mycosis is usually failure of antibiotic therapy (especially when virus infections have been excluded). Immunocompromised patients (AIDS, cancer, malnutrition, post-transplantal and post-operation stages are among the main risk factors) present the highest risk of acquiring visceral mycoses.

Several microfungi (especially of the genera Aspergillus, Fusarium, etc.) produce mycotoxins often causing dangerous mycotoxicoses affecting diverse organs of vertebrates (including man): liver, intestine, CNS, kidneys or the reproductive system.

The traditional diagnosis of fungal diseases is based on microscopic examination of samples originating from lesions (crusts, scrapings, scales of skin and nails) after their partial digestion in $10 \% \mathrm{KOH}$. The microscopy is supplemented with cultivation of the samples, frequently on Sabouraud agar (SGA) with chloramphenicol and cykloheximide simultaneously at temperatures $25-30^{\circ} \mathrm{C}$ and $37^{\circ} \mathrm{C}$ for up to 2-3 weeks; some yeasts or visceral fungi require richer media such as BA or BHI agar. The macroscopic pattern of the fungal colony and microscopic morphology of the fungus is evaluated. In addition, biochemical identification sets are generally used for the determination of pathogenic yeasts (Candida spp., Cryptococcus neoformans). Histological examinations are also applied in invasive visceral mycoses (e.g. histoplasmosis, blastomycosis) - the sections are usually stained according to Schiff's or the more specific Grocott and Gomori methods. Serological examinations of the patients include RDPA, counter immunoelectrophoresis, agglutination and ELISA. Latex agglutination or ELISA is generally used for the detection of antigen. Molecular biology methods (PCR) have evolved into progressive diagnostic tools in recent years.

\subsubsection{Family Arthrodermataceae [Order Onygenales, Class Ascomycetes]}

\section{Trichophyton mentagrophytes, T. quinckeanum, T. erinacei}

Teleomorph: Arthroderma benhamiae. 
Source of infection (natural host range): rodents (mouse, black rat, brown rat, muskrat, nutria, squirrel), hedgehog (Erinaceus europaeus, Atelerix albiventris - T. erinacei) and other mammals.

Animal disease: inapparent course, or trichophytia (favus) in some animals.

Transmission mode: by direct or indirect contact (zoophilic dermatophytes).

Human disease: dermatophytosis (trichophytia, favus).

Bio-containment: BSL-2.

Diagnosis: symptoms; microscopy of affected epidermis, hair and nails samples (preparate in $10 \% \mathrm{KOH}$ ), cultivation (SGA with chloramphenicol and cykloheximide).

Treatment: topical fungicide ointments; oral fungicides (griseofulvin, itraconazole).

Prevention: vaccine for animals, e.g. Trichopelen (Czech Bioveta).

Geographical distribution: worldwide.

\section{Trichophyton verrucosum}

Source of infection (natural host range): cattle (a typical zoophilic dermatophyte).

Animal disease: trichophytia (ringworm).

Transmission mode: by contact (arthrospores in hairs may be viable for more than 1 year).

Human disease: dermatophytosis (trichophytia).

Bio-containment: BSL-2.

Diagnosis: see prior species.

Treatment: griseofulvin perorally, topically imidazoles (econazole, ketoconazole etc.), Hexadecyl spray.

Prevention: vaccine Trichoben (Bioveta) for calves.

Geographical distribution: worldwide.

\section{Trichophyton simii}

Teleomorph: Arthroderma simii.

Source of infection (natural host range): primates, occasionally domestic fowl.

Animal disease: dermatophytosis.

Transmission mode: by contact.

Human disease: dermatophytosis (trichophytia).

Bio-containment: BSL-2.

Diagnosis and therapy: see prior species.

Geographical distribution: India, Africa, South America (Brazil).

\section{Microsporum canis}

Teleomorph: Arthroderma otae. 
Source of infection (natural host range): cat, dog.

Animal disease: inapparent course (symptomless carriage), or else dermatophytosis.

Transmission mode: by contact (zoophilic dermatophyte).

Human disease: dermatophytosis.

Bio-containment: BSL-2.

Diagnosis and therapy: see prior species.

Prevention: vaccine Biocan M (Bioveta).

Geographical distribution: worldwide.

\section{Microsporum persicolor}

Teleomorph: Arthroderma persicolor.

Source of infection (natural host range): rodents, insectivores (hedgehog).

Animal disease: sporadically dermatophytosis.

Transmission mode: by contact.

Human disease: dermatophytosis (microsporia).

Bio-containment: BSL-2.

Diagnosis and therapy: see prior species.

Geographical distribution: Europe, Canada.

\section{*Microsporum gypseum, M. fulvum}

Teleomorph: Arthroderma gypsea, A. fulva, A. incurvata.

Source of infection: soil (a typical geophilic dermatophyte).

Animal disease: sporadic dermatophytosis.

Transmission mode: contact with contaminated soil (e.g., in gardeners).

Human disease: dermatophytosis (microsporia).

Bio-containment: BSL-2.

Diagnosis and therapy - see prior species.

Geographical distribution: worldwide.

\subsubsection{Family Gymnoascaceae [Order Onygenales]}

\section{*Coccidioides immitis, C. posadasii}

Closely related species. The teleomorph has not yet been recognized, but according to recent molecular studies of the anamorph it could belong to this family (because the close genomic relationship with Uncinocarpus reessi, a gymnoascacean fungus). A dimorphic fungus: filamentous form with arthrospores 4-6×2-3 $\mu \mathrm{m}$; but spherules with diameter $20-60 \mu \mathrm{m}$ containing endospores $2-5 \mu \mathrm{m}$ in the tissues. 
Source of infection: soil in arid and semi-arid areas (mainly in and around rodent burrows).

Animal disease: inapparent course or bronchopneumonia.

Transmission mode: aerogenic - by inhalation of arthrospores (released from the soil during strong winds, dusty storms, ground works, earthquakes), highly contagious agent. Frequent infections in persons occurring in natural foci of the disease (farmers, military personnel, tourists). Positive intradermal tests have been found in inhabitants of endemic areas at high prevalence rates (up to $90 \%$ ).

Human disease: coccidioidomycosis - primarily febrile pulmonary disease with skin erythema; about $60 \%$ of cases are subclinic, but dissemination (progressive form) may occur in approximately $1 \%$ of patients with the fungus dissemination into different organs including skin, bones or CNS, with fatality rate of $40 \%$ in the treated disseminated form. Very severe course of infection is described in immunocompromised patients; increased susceptibility and higher risk for disseminated coccidioidomycosis is also observed in Philippinos, Afroamericans and Indians. A total of 4,500 new cases have been recorded in California in 1992 (majority of cases in San Joaquin Valley: the disease is sometimes called "Valley fever" in America). Human incidence rate in the area of Tulare (California) has been evaluated as $41 / 100,000$ population in 1991 , and up to $54 / 100,000$ population in Arizona during 1998-1999. A total of c. 900 out of 5,300 inmates at the central Californian "Pleasant Valley State Prison" contracted coccidioidomycosis in the years 2004-2006, and at least 12 died from it. The whole California reported c. 3,000 cases of Valley fever in 2006. Several imported cases of coccidioidomycosis have been reported annually in Europeans returning from a visit to endemic US areas (probably underreported due to underdiagnosis).

Bio-containment: BSL-3 (a high risk of laboratory-acquired infection).

Diagnosis: microscopy (biopsy, sputum, pus, CSF: spherules), serology (CFT, precipitation), intradermal test (coccidioidin); cultivation (SGA).

Treatment: amphotericin B, ketoconazole (not in the CNS forms), fluconazole, itraconazole, triazole.

Prevention: vaccine, but insufficiently available.

Geographical distribution: southwestern USA (xerothermic, mainly desert areas - California, Nevada, Arizona, Utah, New Mexico and south Texas), Mexico, less often semiarid areas of Central and South America (e.g. Venezuela, Brazil).

\subsubsection{Family Ajellomycetaceae [Order Onygenales]}

\section{${ }^{*}$ Histoplasma capsulatum, H. duboisii}

Teleomorph: Ajellomyces capsulatus. A dimorphic fungus with a filamentous conidial form in the saprophytic phase, while a yeast-like budding form in the host's tissue; intracellular parasite. 
Source of infection: soil with droppings of birds (starling, American blackbirds, cowbirds, oilbird) and bats in their communal roosting sites and gathering places (caves, parks, groves etc.).

Animal disease: inapparent course or lymphoreticulitis, enteritis; sporadically death in dogs. A closely related species, H. farciminosum, causes histoplasmosis in horses.

Transmission mode: aerogenic (ground works on bird roosting sites, demolition of old buildings, visits of caves contaminated with bat and oilbird droppings).

Human disease: histoplasmosis - primarily a pulmonary disease characterized with pneumonia and lymphadenopathy, similar to tuberculosis (lung calcification), with a good prognosis; however, generalized or progressive clinical forms (approximately 1\% of all cases) may result in hepatosplenomegaly, the CNS infection, ulceration of digestive tract and other severe manifestations with high lethality. Pulmonary histoplasmosis might be chronic, and with relapses. The course of disease is severe in immunocompromised patients (e.g., AIDS) as well. H.d. is the agent of African histoplasmosis with predominantly subacute course and formation of cutaneous or bone granulomas without lung affection.

Bio-containment: BSL-3.

Diagnosis: chest X-ray, microscopy (Gram and Giemsa staining) and cultivation of sputum or tissue lesions (SGA, $25-30^{\circ} \mathrm{C}$ : production of characteristic conidia, and demonstration of conversion of isolate $\mathrm{M} \rightarrow \mathrm{Y}$ at $37^{\circ} \mathrm{C}$ ), liver or lung biopsy in severe forms (microscopy-silver stain); serology (RDPA, ELISA, CFT, IFA, latex agglutination), intradermal test (histoplasmin retrospective demonstration of exposition).

Treatment: amphotericin B, ketoconazole itraconazole.

Geographical distribution: nearly worldwide. $H$. capsulatum is relatively frequent in the USA (natural foci are present in e.g. the basin of Mississippi and Ohio rivers), Central and South America, Africa, India and southeastern Asia (e.g., first indigenous case reported in Taiwan in 2005), while infrequent occurrence has been reported in Europe where, e.g., 118 cases were documented in 1995-1999 (but only eight obviously autochthonous in Italy, UK, Turkey and Germany); H. duboisii has been recorded in Africa only.

\section{*Bastomyces dermatitidis}

Teleomorph: Ajellomyces dermatitidis. A dimorphic fungus: normally filamentous, but yeast-like forms are produced in the tissue at $37^{\circ} \mathrm{C}$.

Source of infection: soil; dog (equids). The specific reservoir (ecological niche) unknown, but the fungus habitat seems to be associated with waterways in North America.

Animal disease: ulcerative changes.

Transmission mode: aerogenic, by contact. 
Human disease: (North American) blastomycosis - pulmonary, occasionally cutaneous, mucocutaneous as well as generalized (disseminated) forms with high lethality.

Bio-containment: BSL-2/3.

Diagnosis: chest X-ray, microscopy of sputum, pus and biopsy (yeast-like forms), cultivation (SGA), serology (RDPA, ELISA).

Treatment: amphotericin B, ketoconazole itraconazole.

Geographical distribution: North America. Some cases reported in Africa, Asia and Europe.

\section{*Emmonsia crescens}

Teleomorph: Ajellomyces crescens. A dimorphic fungus, very closely related to genera Histoplasma and Blastomyces (based on 18S rRNA sequences), with small spherical to oval conidia $(2-3 \mu \mathrm{m})$ in saprophytic phase, and with thick-walled spherules called adiaspores with a diameter up to 500 (700) $\mu \mathrm{m}$ (whereas close species Emmonsia parva, with unproven pathogenicity for man, has spherules only up to $40-50 \mu \mathrm{m}$ ) in the lung tissue; granulomas.

Source of infection: soil (rhizosphere), burrows of mammals.

Animal disease: pulmonary emmonsiosis (adiasporomycosis, adiaspiromycosis) of mammals, mainly in rodents. Emmonsiosis caused by E. parva occurs in steppe and desert rodents.

Transmission mode: aerogenic (from the soil). Enhanced occupational risk of infection (farmers and persons handling with soil-diggers).

Human disease: pulmonary emmonsiosis (adiasporomycosis, adiaspiromycosis), occasionally with fever, cough, dyspnoea, exceptionally with lethal course. A number of severe cases of the disease have been recorded in Brazil and Argentina. Several benign cases have been documented in the former Czechoslovakia.

Bio-containment: BSL-2.

Diagnosis: chest X-ray, lung biopsy (adiaspores), cultivation (SGA).

Treatment: amphotericin B, ketoconazole.

Geographical distribution: worldwide (E. crescens).

\section{*Paracoccidioides brasiliensis}

Teleomorph: supposedly Ajellomyces (based on 18S rRNA sequences of the anamorph). A dimorphic fungus: filamentous, but multipolarily budding yeast-like form in the tissue.

Source of infection: soil, plant substrates. A number of isolates have been obtained from the armadillo Dasypus novemcinctus in Brazil; armadillos obviously play an important role in the ecology of this fungus (hosts, reservoir?), but they are uncertain as the source of human infection. 
Transmission mode: aerogenic (farmers), by contact, alimentary.

Human disease: paracoccidioidomycosis (South American blastomycosis) the fungus invades either nasal and oral mucosa and skin (mucocutaneous form: ulcerative granulomas) or predominantly lymphatic system (the lymphatic form), eventually disseminate (the visceral form, affecting the lungs, intestine, liver).

Bio-containment: BSL-2/3.

Diagnosis: microscopy (tissue, pus, sputum), biopsy, cultivation (SGA); RDPA.

Treatment: amphotericin B, ketoconazole.

Geographical distribution: South and Central America.

\section{${ }^{*}$ Lacazia loboi}

The fungus is genomically closely related to $P$. brasiliensis.

Source of infection: marine ecosystem - vegetation, soil; dolphins (?).

Transmission mode: contact - percutaneous (skin traumatized by plant thorns or insect bites).

Animal disease: lobomycosis in bottlenose dolphins (Tursiops truncatus, T. aduncus) and Guiana dolphin (Sotalia guianensis).

Human disease: lobomycosis - chronic, granulomatous lesions on the skin and in subcutaneous tissues, similar as in dolphins.

Bio-containment: BSL-2.

Diagnosis: microscopy (biopsy and histopathology: spherical, thick-walled and yeast-like cells in the tissue); the organism has not yet been cultured in vitro.

Treatment: surgery; clofazimine with or without itraconazole, and with fluorocytosine.

Geographical distribution: coastal areas in the South and Central America, less common in southern USA (Florida, Carolina), South Africa, and exceptionally in Europe.

\subsubsection{Family Ophiostomataceae [Order Ophiostomatales]}

\section{${ }^{*}$ Sporothrix schenckii}

Teleomorph: Ophiostoma. A dimorphic fungus: filamentous, but usually yeast-like forms occur in the tissue.

Source of infection: soil, plant remnants, cornstalks, woody plants; sphagnum moss, less often cat.

Animal disease: furunculous dermatitis, lymphadenitis, sometimes disseminated form. 
Transmission mode: by contact (injury by thorns and splinters; or scratching/biting by infected cats: 24 cases in Brazil); less often aerogenic (an outbreak in South-African mines).

Human disease: sporotrichosis - chronic pyogenic granulomatous disease of the skin, subcutis, mucosa and lymph nodes. A number of cases of miners in South African (diamond and gold) mines (occupational disease). A total of 41 human cases have been reported in Australia between 2000 and 2003 (the source was contaminated hay). In 1998, a large epidemic occurred in Brazil which continued to 2004 with a total of 759 human cases (the source were infected cats).

Bio-containment: BSL-2.

Diagnosis: microscopy (biopsy), cultivation (SGA).

Treatment: potassium iodide (very effective by local use), amphotericin B (in disseminated forms, i.v. application).

Geographical distribution: mainly (sub)tropics (America, Africa), Australia, rarely in Europe.

\subsubsection{Family Eurotiaceae [Order Eurotiales]}

\section{Penicillium marneffei}

It is the only dimorphic (yeast-like arthrospores in the tissue) and pathogenic species of Penicillium.

Source of infection (natural host range): large bamboo rat (Rhizomys sumatrensis) and lesser bamboo rat (Cannomys badius).

Animal disease: inapparent course.

Transmission mode: aerogenic.

Human disease: disseminated penicillosis (high fever, skin lesions, intestinal, pulmonary etc. symptoms), more often in immunocompromised patients (HIV); very high fatality rate $-75 \%$ in treated patients, $100 \%$ in untreated cases.

Bio-containment: BSL-2/3, highly contagious.

Diagnosis: microscopy and cultivation of samples from biopsy.

Treatment: amphotericin B, itraconazole.

Geographical distribution: Southeast Asia, India (state Manipur), China (Guangdong province).

\section{*Aspergillus fumigatus, A. flavus}

Source of infection: plant remnants, hay, compost (thermotolerant fungi).

Animal disease: pulmonary aspergillosis (pneumomycosis); abortion of cattle; aflatoxicosis. 
Transmission mode: aerogenic; alimentary intoxicosis (aflatoxins: A. flavus contaminated nuts, dried tropical fruit, succade etc.).

Human disease: pulmonary aspergillosis (A. fumigatus), or aspergilloma (colonization of lung cavities with mycelium, encapsulation); secondary dissemination and invasion of the kidney, heart (endocarditis), CNS and paranasal cavities (granulomas), spleen, liver, stomach, mainly in immunocompromised persons (e.g., with tuberculosis, AIDS or cancer); allergic aspergillosis (hypersensitivity in persons with atopic eczema and asthma, then "syndrome of maltster's lungs" - infection originates from barley); aspergillosis is the most frequent human visceral mycosis, with fatality rate of about $10 \%$; further aflatoxicosis (especially A. flavus - aflatoxins damage the liver and are cancerogenic).

Bio-containment: BSL-2.

Diagnosis: microscopy (sputum, biopsy), cultivation (SGA without cycloheximide, Czapek-Dox agar), serology (RDPA, latex agglutination, electrophoresis, ELISA), intradermal test.

Treatment: potassium iodide, amphotericin B, ketoconazole, itraconazole, voriconazole and newly posaconazole; surgical resection of wounded tissue (aspergilloma); corticosteroids (allergic aspergillosis).

Geographical distribution: worldwide.

\subsubsection{Family Hypocreaceae [Order Hypocreales]}

\section{${ }^{*}$ Fusarium solani, F. oxysporum, F. moniliforme}

Characteristically curved (crescent-shaped) septal macroconidia. Teleomorphs belong to ascomycetous genera Gibberella, Nectria etc.

Source of infection: plant remnants.

Animal disease: fusariosis; also fusariotoxicosis (the toxins are fumonisine B1, zearalenone, deoxynivalenon - they damage nervs, kidney, lungs, heart, uterus).

Transmission mode: by contact (percutaneously, via conjuctivae).

Human disease: fusariosis - keratomycosis (ocular infection), onychomycosis. For instance, about 100 cases of Fusarium keratitis were reported in people using contact lenses from Singapore and Hongkong in 20052006, and a similar disease (130 cases) was notified in USA in the same 2 years.

Bio-containment: BSL-2.

Diagnosis: microscopy of the affected tissue, cultivation.

Treatment: amphotericin B.

Geographical distribution: worldwide. 


\subsubsection{Order Dothideales [Class Ascomycetes]}

\section{*Madurella mycetomi, M. grisea, Scedosporium apiospermum, Exophiala jeanselmei, Leptosphaeria senegalensis, Phialophora spp., Neotestudina rosatii}

Teleomorph of S. apiospermum is Pseudallescheria boydii (Microascaceae). Teleomorph of L. senegalensis belongs to Dothideales.

Source of infection: soil, plants.

Animal disease: maduromycosis.

Transmission mode: by contact, percutaneously (injury) - people working on fields (farmers).

Human disease: maduromycetoma, eumycetoma (mycotic mycetoma) - granulomas and abscesses with fistula in skin and subcutis, rarely on bones, sometimes dissemination; it is necessary to differentiate this disease from mycetoma caused by actinomycetes (i.e. actinomycetoma).

Bio-containment: BSL-2.

Diagnosis: microscopy (pus from fistula, biopsy), cultivation (SGA without cycloheximide etc.).

Therapy (less effective): ketoconazole, amphotericin $\mathrm{B}$; resection of the affected tissue.

Geographical distribution: tropics and subtropics (America, Africa, Asia, Australia).

\subsection{8 “Family” Dematiaceae [Class Hyphomycetes]}

Teleomorphs mostly unknown at present.

\section{*Phialophora verrucosa, Fonsecaea compacta, F. pedrosoi, Cladophialophora carrionii, Exophiala jeanselmei, Rhinocladiella aquaspersa}

Teleomorphs unknown at present.

Source of infection: plant remnants.

Animal disease: chromoblastomycosis.

Transmission mode: by contact (percutaneously) - farmers.

Human disease: chromoblastomycosis (chromomycosis) - chronic disease of skin and subcutis characterized by verrucose lesions covered with scurfs (mainly on extremities, especially on legs).

Bio-containment: BSL-2.

Diagnosis: microscopy of the tissue (typical brown spherical multiseptate cells and brown hyphae), cultivation (SGA). 
Treatment: amphotericin B, fluorocytosine.

Geographical distribution: worldwide, predominantly areas with warm climate.

\section{* Exophiala dermatitidis, E. spinifera, Ochroconis gallopavum, Phialophora spp., Cladophialophora bantiana, Scedosporium proliferans, Ramichloridium mackenziei, Drechslera spp., Curvularia spp., Alternaria alternata, Aureobasidium pullulans}

Teleomorphs, if known, belong to ascomycetes.

Source of infection: plant remnants.

Animal disease: phaeohyphomycosis.

Transmission mode: percutaneous.

Human disease: phaeohyphomycosis - nonspecific solitary lesions in subcutis, rarely inside the affected tissue, keratitis, occasionally a disseminated infection (cerebral abscesses).

Bio-containment: BSL-2.

Diagnosis: microscopy of wounded tissue, cultivation (SGA).

Treatment: amphotericin B, ketoconazole; surgical intervention (excision).

Geographical distribution: worldwide.

\section{${ }^{*}$ Hortaea (Exophiala) werneckii}

Source of infection: soil (a halophilic saprophyte).

Animal disease: unknown.

Transmission mode: percutaneous.

Human disease: tinea nigra palmaris - dark lesions localized on the palm of one or both hands, occasionally on the sole.

Bio-containment: BSL-2.

Diagnosis: microscopy of wounded tissue, cultivation (SGA).

Treatment: ketoconazole.

Geographical distribution: only in tropical and subtropical areas.

\section{*Acremonium kiliense, A. recifei, Scytalidium hyalinum}

Source of infection: plant remnants.

Animal disease: hyalohyphomycosis.

Transmission mode: percutaneous.

Human disease: hyalohyphomycosis - usually ulceratose and nodulose lesions in skin and subcutis, keratitis (an uncommon disease).

Bio-containment: BSL-2.

Diagnosis: microscopy of wounded tissue, cultivation (SGA).

Treatment: ketoconazole.

Geographical distribution: worldwide. 


\subsubsection{Family Mucoraceae [Order Mucorales, Class Zygomycetes]}

\section{*Absidia corymbifera, Apophysomyces elegans, Rhizopus oryzae, Rhizomucor pusillus, Mucor spp.}

Source of infection: dead plant remnants and other abiotic substrates.

Animal disease: mucormycosis of gastrointestinal tract, abortion.

Transmission mode: alimentary, aerogenic.

Human disease: mucormycosis (zygomycosis) - cutaneous (wound infections), gastrointestinal, cranial, pulmonary or disseminated; the most serious and often fatal is rhinocerebral zygomycosis (spreading from nasal mucosa through nasal cavities and orbit into the brain; predisposition factors: diabetes, leukaemia, lymphoma, uraemia, immunosuppression).

Bio-containment: BSL-2.

Diagnosis: microscopy (biopsy: wide non-septate hyphae), cultivation (SGA); often diagnosed only post mortem at autopsy.

Treatment: amphotericin B (itraconazole, posaconazole, caspofungin); iron chelation, hyperbaric oxygen, cytokine therapy; resection of the necrotic tissue.

Geographical distribution: worldwide, ubiquitous.

\subsubsection{Family Entomophthoraceae [Order Entomophthorales]}

\section{*Basidiobolus ranarum, Conidiobolus spp., Entomophthora}

Source of infection: plant remnants, water, soil; reptiles, amphibians.

Animal disease: entomophthoromycosis (phycomycosis).

Transmission mode: by contact (traumatic inoculation), exceptionally also alimentary.

Human disease: entomophthoromycosis (phycomycosis) - subcutaneous and mucosal (nasal) tumor-like changes.

Bio-containment: BSL-2.

Diagnosis: microscopy (biopsy: hyphal elements sparse and often fragmentary; occasional septations within hyphae - contrary to mucormycosis), cultivation.

Treatment: potassium iodide, amphotericin B.

Geographical distribution: worldwide, but mostly in Africa, India and South America.

\subsubsection{Family Filobasidiaceae [Order Filobasidiales (Sporidiales), Class Heterobasidiomycetes]}

\section{*Cryptococcus neoformans, $C$. gattii}

Teleomorph: Filobasidiella neoformans. C. neoformans complex produces spherical yeast-like cells $2-10 \mu \mathrm{m}$ with a mucopolysaccharide capsule; serotypes A to D. 
Earlier regarded as two closely related subspecies of $C$. neoformans, today they are treated as separate species; $C$. gattii corresponds to the serotypes B and C. The species $C$. neoformans is now differentiated into two variants: $C$. neoformans var. grubyi (serotype A), and C. neoformans var. neoformans (serotype $\mathrm{D}$, and hybrid serotype AD).

Source of infection: soil contaminated with bird droppings in C. neoformans (faeces of pigeons and some ornamental birds present a nutritive substrate for the growth of serotypes $\mathrm{A}, \mathrm{D}$, and $\mathrm{AD}$; pigeon excreta may contain as many as about $5.10^{7}$ of $C$. neoformans cells per gram). In C. gattii, some species of trees (mainly eucalyptus) - leaves, bark, wood chips, decaying wood or rhizosphere present the fungus habitat, i.e. reservoir of infection.

Animal disease: cryptococcosis - pneumonia, meningitis; C. gattii: disease in koalas, cats, dogs, ferrets, and other mammals.

Transmission mode: aerogenic by inhalation; less often by contact. Increased risk of transmission in persons, who come in contact with contaminated bird (largely feral pigeon) droppings (occupational exposure: road sweepers, workers on roofs, lofts and garrets, diggers).

Human disease: cryptococcosis - chronic to subacute pulmonary, skin or disseminated form (meningoencephalitis), with the fatality rate about 20-30\%; often in patients suffering from AIDS and other immunocompromising diseases. Small discrete nodules, scarification and encapsulation of focuses similar to those in tuberculosis may occasionally occur in pulmonary form of the disease. While cryptococcosis caused by $C$. neoformans commonly occurs in immunocompromised patients, the disease due to $C$. gattii largely occurs in immunocompetent persons and causes headache, persistent cough, shortness of breath, and the case fatality rate is about $9 \%$. For instance, 272 cases (as of July 2010) of predominantly pulmonary cryptococcosis caused by $C$. gattii highly virulent genotype VGII have been reported on the Vancouver Island and adjacent inland areas in British Columbia (Canada) since 1999; and after 2003, the disease has spread to northwest U.S. states Washington, Oregon, California and Idaho, causing 60 human cases (15 ended in death), as reported in July 2010, in 2005-2006.

Bio-containment: BSL-2.

Diagnosis: microscopy (Indian ink negative preparation for detection of capsula) and cultivation (SGA or selective agar with Guizotia) of CSF, pus, urine, prostatic excretion; serological demonstration of polysaccharide antigen in CSF (CFT, latex test, ELISA).

Treatment: combination of amphotericin B (the most effective is its liposomal form) + flucytosine, ketoconazole, fluconazole.

Geographical distribution: C. neoformans worldwide. C. gattii is mainly distributed in tropical and subtropical areas: Australia, New Zealand, Papua New Guinea, southeastern Asia, India, central and southern Africa, South America (Brazil), Mexico, south California, but lately also in British 
Columbia and northwestern USA, occasionally southern and central Europe (Spain, France, Italy, Greece, Austria, Germany).

\subsubsection{Order Ustilaginales [Class Heterobasidiomycetes]}

\section{$\left({ }^{* *}\right)$ Pneumocystis jirovecii (Synonym P. carinii)}

Formerly classified within Protozoa, but the rRNA analysis revealed close similarity with smut fungi.

Source of infection (natural host range): brown rat and other rodents, domestic animals (dog); man (there are anthroponotic and zoonotic strains of this species).

Animal disease: inapparent course (sometimes massive findings of cysts in lungs of rabbits and rodents).

Transmission mode: aerogenic, by contact.

Human disease: pneumocystosis, often in immunocompromised persons (leukaemia, AIDS, malnutrition); generally subfebrile. [Interstitial pneumonia of newborns is an anthroponosis with fatality rate $0 \overline{-40 \% \text { (aetiology }}$ of this disease was elucidated by Vaněk and Jírovec in 1952)].

Bio-containment: BSL-2.

Diagnosis: microscopy of sputum (IF determination of antigen), bronchoalveolar lavage, lung biopsy. Giemsa or silver staining of the samples: mature "cysts" (in fact basidia) are 5-8 $\mu \mathrm{m}$ in diameter with double wall and contain eight uninuclear bodies, "sporozoites" (in fact basidiospores), and spots of eosinophilic substance with aggregations of very small spherical forms 1-4 $\mu \mathrm{m}$, called "trophozoites"; PCR, sometimes serology (CFT, IFA).

Treatment: cotrimoxazole, trimethoprim + sulphamethoxazole, pentamidine, sulphadiazine + pyrimethamine.

Geographical distribution: worldwide.

\subsubsection{Family Pythiaceae [Order Peronosporales, Class Oomycetes]}

\section{*Pythium insidiosum}

A plant pathogen (in nature, biflagellate zoospores attach the water plants), not a true fungus.

Source of infection: water.

Animal disease: a cutaneous and pulmonary disease in domestic mammals (cat, dog, horse, cattle), with chronic ulcerated lesions on the limbs, chest, and abdomen. 
Transmission mode: by contact (skin abrasions - e.g., in rice fields).

Human disease: pythiosis - ocular form (keratitis), cutaneous and subcutaneous form, and arterial form with chronic ulcers on the legs, ischaemia, thrombosis of major arteries, and necrosis (fatality rate of c. $40 \%$ in the arterial form).

Bio-containment: BSL-2.

Diagnosis: microscopy (biopsy: hyaline, thin-walled hyphae and hyphal fragments resembling zygomycetes - the cell walls are non-parallel), cultivation (e.g., SGA), serology (IFA, immunodiffusion).

Treatment: surgery combined with antifungal compounds (amphotericin B, azoles); in ocular pythiosis, keratoplasty is usually necessary but not always it is successful.

Geographical distribution: tropical and subtropical regions (reported from Thailand, Malaysia, Australia, New Zealand, Haiti).

\subsection{Protozoa}

\subsubsection{Family Trypanosomatidae [Order Kinetoplastida, Class Kinetoplasmidea]}

\section{Trypanosoma cruzi}

Flagellates of two genotypes (I and II), and two life forms: trypomastigotes $15-20 \times 2 \mu \mathrm{m}$ with an undulating membrane (operates as a traction propeller) and kinetoplast (a specialized mitochondrium near the base of flagellum); replicating spherical amastigotes $(2-6 \mu \mathrm{m})$ occur in the tissue (RES and muscle cells) most of them generate new trypomastigotes that disseminate via the blood.

Source of infection (natural host range): dog, cat, pig, goat, cattle, rabbit, rodents (Neotoma spp. woodrats, guinea pig), armadillo (Dasypus), opossum (Didelphis albiventris, reservoir), punaré (Trichomys apereoides, reservoir), racoon (Procyon), bats, monkeys; man (sporadically).

Animal disease (dog): chronic myocarditis, meningoencephalitis, anaemia.

Transmission mode: bites of tropical haematophagous insects - kissing bugs (Reduviidae): Triatoma infestans, T. sanguisuga, Rhodnius prolixus, Meccus longipennis, T. rubida (USA), etc. (trypanosomes develop in their gut and are rubbed with the bugs' excrements into wounds or conjunctiva). Other means of transmission include blood transfusion, organ transplantation, or congenital infection in childbirth, and also alimentary route - 58 food-borne human cases were reported in Brazil in 2007 and 2009, after eating "acai", a food prepared from palm tree of the family Aracaceae (fruit, juice). A similar large outbreak also occurred in children from a school in Venezuela: the palm 
juice was apparently contaminated with triatomid bugs (their excrements). Most of the Amazonian population consumes acai juice daily.

Feral cycle: exoanthropic (wild) mammals $\rightarrow$ kissing bugs $\rightarrow$ mammals.

Domestic cycle: synanthropic mammals, human $\rightarrow$ kissing bugs.

Peridomestic cycle: involves domestic animals.

Human disease: Chagas disease, American trypanosomiasis - a chronic general disease with cardiomyopathy and CNS affection, it starts after an 1-2 week incubation period; initial symptoms include occuloglandular syndrome called Romaña's sign in 50\% of infected people (one-sided conjuctivitis and swelling of the eyelid), enlargement of regional lymph nodes, irregular febrile periods, myalgia; the fatality rate is about $8 \%$ in children (myocarditis, meningoencephalitis), lower lethality in adults. Chronic forms (sometimes latent period of 10-20 years): heart arhythmia, dilatation of heart, esophagus ("megaoesoophagus"), gut ("megacolon”), and hepatosplenomegaly; the death usually occurs after perforation ("explosion") of the dilated gut or heart. An estimated 18 million persons are infected in Central and South America, and 15,000-50,000 of them die annually. For example, an average of $50 \%$ of Bolivian population was infected in 1994, while the infection rate in endemic areas was as high as $80-90 \%$. Morbidity due to Chagas disease in Honduras was estimated as 5,172/100,000 population in 1996. In 2007, a major alimentary outbreak occurred in Venezuela (1,000 exposed persons; $75 \%$ of the cases were observed among students 16 -year old or younger; $75 \%$ were symptomatic, $28 \%$ with cardiac involvement, one child died of acute myocarditis). The disease is ancient: DNA of $T$. cruzi was detected in 4,500-9,000-year old mummies of Indians.

Bio-containment: BSL-2.

Diagnosis: microscopy - blood smear, thick drop (Giemsa staining: flagellar promastigote or trypomastigote phase), biopsy (lymphatic glands: oval unflagellar amastigote phases in tissues and cells of RES - pseudocysts $5 \times 1.5 \mu \mathrm{m}$ with spherical nucleus and rod-shaped blepharoblasts), intraperitoneal inoculation of infected blood to guinea pig (or to Syrian hamster or suckling mouse), cultivation (enriched BA, so-called NNN agar NovyMcNeal-Nicoll), serology (CFT, RIHA, IFA, ELISA, latex agglutination), intradermal test. Xenodiagnostics may also be used (uninfected kissing bugs from a laboratory rear are fed on patients, after 3-4 weeks the gut content of the bugs is examined for trypanosomes).

Treatment (difficult - no satisfactory drugs are available): nitrofurfuryliden (Nifurtimox), benzonidazole, isometamidium derivates.

Prevention: vaccine unavailable. Control of triatomine bugs (spraying the walls and roofs of houses, self-protection).

Geographical distribution: Central and South America, Mexico, southern USA (a rare human disease in Arizona - a total of 7 cases were reported, but $41 \%$ of kissing bugs collected in Tucson were found infected with T. cruzi in 2006; occasionally also Louisiana, Texas, Tennessee, and California). 


\section{Trypanosoma brucei}

Flagellates (15)20-30(40) $\times 1.5-3 \mu \mathrm{m}$ with an undulating membrane. Two subspecies: zoonotic $\boldsymbol{T}$. $\boldsymbol{b}$. rhodesiense, and anthroponotic ${ }^{* *} \boldsymbol{T}$. $\boldsymbol{b}$. gambiense, formerly classified as two separate species. They are characteristic by their "immune evasion" strategy due to antigenic variation within a host, caused by switching of surface glycoproteins (VSG genes are responsible).

Source of infection (natural host range): in $T$. $b$. rhodesiense antelopes (Tragelaphus, Sylvicapra), cattle, sheep, goat, swine, dog, African buffalo, warthog, elephant, rhinoceros, hippopotamus, lion, hyena and other wild mammals (reservoir), crocodile (T. b. rhodesiense); in T. b. gambiense mainly human; but also domestic pig, dog, cattle, sheep, putty-nosed monkey (Cercopithecus nictitans), some rodents and carnivores.

Animal disease: inapparent course [the closely related $T$. b. brucei causes disease of cattle called "nagana", and T. congolense fever of cattle named "gambia"; these both trypanosomes are however non-pathogenic for man].

Transmission mode: tsetse flies (via infected saliva: Glossina morsitans [savannah ecosystem] - T. b. rhodesiense; G. palpalis and G. tachinoides [river habitats] - T. b. gambiense). Tsetse flies remain infective during their whole life (up to 6 months), the development of trypanosomes into infective stage takes about (12)20-30(40) days.

Human disease: African sleeping disease, African trypanosomiasis (EastAfrican - T. $b$. rhodesiense, or West-African - T. b. gambiense) - irregular high fever attacks without chills, hyperhidrosis, headache, tachycardia, respiratory distress, skin rash, anaemia at the beginning, later lymphadenitis (swollen lymph nodes in the hind trianguloid part of the neck and axillae, called Winterbottom's sign), swollen eyelids, oliguria, neurologic signs (hypersensitivity for touch, hypersomnia, meningoencephalitis); hepatosplenomegaly; acute course (2-4 months: $T$. $b$. rhodesiense) or chronic (1-6 years: T. b. gambiense). Fatality rate is high (90\%), 1-2 years after primary infection without specific therapy the patient dies. Re-emergence of the disease has occurred during last years (DR Congo etc.: T. b. gambiense). The annual incidence of sleeping sickness in sub-Saharan Africa is estimated as 50,000-70,000 cases.

Bio-containment: BSL-2.

Diagnosis: microscopy - thick drop, blood smear, smear of punctate from swollen lymphatic nodes or from CSF (native preparation, Giemsa, IF), serology (IFA, CFT, AR, RDPA, ELISA, RIHA), cultivation (agar), inoculation of laboratory rat or mouse (T. b. rhodesiense); novel molecular techniques such as PCR and proteome fingerprinting.

Treatment: pentamidine (ineffective in late-stage of disease, less effective against $T . b$. rhodesiense) in acute phase or as a prophylaxis; suramin (a trypanocidal agent that is however toxic, causing degeneration of the liver, 
kidney and adrenal glands), arsenic preparates (toxic) in chronic phase (CNS affection) such as melarsoprol (the effectivity of this drug has been reduced by some $20 \%$ in the last years as demonstrated in DR Congo), tryparsamide, and the latest $\alpha$-difluoromethylornithine (DFMO), being relatively nontoxic and effective in combination with nifurtimox even in therapy of the CNS phase of the disease.

Geographical distribution: tropics of East Africa - savannah (T. b. rhodesiense), West and Central Africa - river basins (T. b. gambiense); both subspecies occur simultaneously only in Uganda.

\section{Leishmania major, L. tropica, L. aethiopica, L. donovani, L. infantum, L. mexicana, L. braziliensis, L. chagasi, L. peruviana, L. killicki}

Flagellates without the undulating membrane (promastigote form 14-25 $\times 1.5$ $3 \mu \mathrm{m}$, of the morphological type of "leptomonas"), intracellular parasites of RES cells (dividing spherical amastigote form 2-5-5 $\times 1.5-2 \mu \mathrm{m}$, with a nucleus and kinetoplast) of vertebrates. The taxonomy of leishmaniae is complicated: L. donovani complex includes three species (L. donovani, L. infantum, and L. chagasi); $L$. mexicana complex also encompasses three main species (L. mexicana, L. amazonensis, and L. venezuelensis); L. tropica; L. major; L. aethiopica; and the subgenus Viannia is represented with four main species: L. (V.) braziliensis, L. (V.) guyanensis, L. (V.) panamensis, and L. (V.) peruviana.

Source of infection (natural host range): in cutaneous leishmaniasis rodents (gerbils Rhombomys, Meriones, Psammomys in desert; rats Mastomys, Arvicanthis, etc. in savannah; punaré Trichomys apereoides in Brazil, hyrax Procavia capensis in Jordan, Israel and Kenya), sloth, kinkajou, also dog, jackal, fox, goat, buffalo, cattle, horse and human in urban cycle; in visceral leishmaniasis canines, rats (Rattus rattus), opposums and man in the urban cycle.

Animal disease: ulcerative inflammations accompanied with changes on skin and mucosa; in dogs also marked cachexia, alopecia, depigmentation of nose and overgrown claws in the cutaneous form, and swollen lymph nodes, ocular signs, epistaxis and kidney failure in the visceral form; canine leishmaniasis is an emerging zoonotic infection in the Mediterranean region, and in North America.

Transmission mode: sandflies (Phlebotomus in the Old World; Lutzomyia in the Americas) - by biting and infectious excrements; in the vector, there are flagellar forms (leptomonas), replicating in its midgut. Within the human host, the promastigote forms are ingested by macrophages where they change into amastigote forms. Main vectors of leishmaniasis in the Mediterranean region are e.g., P. neglectus, P. perfilievi, P. perniciosus, P. papatasi (L. major), $P$. arabicus; elsewhere in the world $P$. martini, $P$. orientalis, $P$. chinensis, P. sergenti (L. tropica), P. duboscqui (L. major), P. alexandri, P. argentipes, 
P. longipes (L. aethiopica), P. pedifer (L. aethiopica), Lutzomyia longipalpis, L. olmeca (L. mexicana), L. wellcomei (L. braziliensis), L. carrerai (L. braziliensis). New experimental molecular data surprisingly indicate possible transmission of $L$. infanti by the dog tick Rhipicephalus sanguineus, including TOT in the tick (Exp. Parasitol. 125: 184-185, 2010). The transmission of leshmaniasis is also possible from man to man by the blood transfusion, inoculation (sharing contaminated needles), or congenitally.

Cycles: exoanthropic (deserts, savannah, tropical rainforests), and synanthropic (domestical, urban).

Human disease: leishmaniasis, a chronic disease with incubation period from a week up to 1 year (sometimes longer in visceral form) and manifesting in several clinical forms: (1) visceral (L. infantum, L. donovani ["kala-azar"], in America L. chagasi) - disease of RES, with fever, hepatosplenomegaly, lymphadenopathy, cachexia, thrombocytopenia, anaemia, leucopenia, hyperglobulinemia and if untreated with a high fatality rate; (2) cutaneous with skin ulcers on face, arms, and legs (L. tropica causes anthroponotic urban form, L. major causes zoonotic form, L. aethiopica - restricted to East Africa; in America L. mexicana causes a disseminated cutaneous form Chicler's ulcer, destructive disease of otis media, L. donovani in Sri Lanka, L. peruviana - Uta disease); two types of cutaneous leishmaniosis are differentiated: (a) urbanus ("dry form") - dry ulcer, late ulcerating, with synanthropic cycle (L. tropica, L. aethiopica); (b) rusticus ("wet form") large acute ulcer, early necrotizing, with exoanthropic cycle (L. major). (3) The mucocutaneous "espundia" form occurs in America and affects nasopharyngeal mucosa with deforming lesions and tissue damage in the mouth, ears or nose that resemble leprosis (L. braziliensis). Leishmania/HIV coinfection is getting an emerging problem worldwide. According to WHO report, 12 million people in the world suffer from leishmaniasis, the number of new cases is about 1.5 million annually and c. 50,000 die. In Africa, the mean yearly number of new cases of visceral leishmaniasis is 19,000 24,000 . However, 100,000 persons died during a massive outbreak of visceral leishmaniasis in south Sudan between the years 1985 and 1987. Ninety percent of visceral leishmaniasis cases occur in Bangladesh, Brazil, India, Nepal and Sudan; 90\% of mucocutaneous leishmaniasis occurs in Bolivia, Brazil and Peru; and 90\% of cutaneous leishmaniasis occur in Afghanistan, Iran, Saudi Arabia, Syria, Brazil and Peru. The annual incidence in Europe (southern only) is much lower, about 750 cases. Asymptomatic infections of humans with Leishmania are 30-100 times more frequent than the clinical cases. DNA of L. donovani was amplified from ancient Egyptian and Nubian mummies 4,000 years old.

Bio-containment: BSL-2.

Diagnosis: microscopy of amastigotes (biopsy - lymph nodes, scrapings, smears: amastigote forms $2.5-5 \times 2 \mu \mathrm{m}$, replicating in monocytes, macrophages; punctate from spleen or sternum in visceral leishmaniasis), cultivation (NNN agar), inoculation of mouse or Syrian hamster (ears, tail), 
serology (IFA, ELISA or haemagglutination test in visceral and mucocutaneous leishmaniasis), intradermal test, PCR.

Treatment: derivatives of pentavalent antimony (natrium stilbogluconate, megluminantimonate, pentamidine), pyrimethamine (Daraprim), amphotericin B (the most effective in liposomal form in visceral leishmaniasis: AmBiosome) or ketoconazole (in cutaneous leishmaniasis), cycloguanyl, miltefosine, $\mathrm{N}$-chlorotaurin (L. donovani); local injections of ulcers, cryotherapy.

Prevention and risk avoidance: human vaccine unavailable at present. LEISHMUNE vaccine is the first licensed vaccine against canine visceral leishmaniasis caused by L. donovani in Brazil. Skin collar with deltametrine can be helpful in dogs. Dog travel to and from endemic areas poses a risk of introducing L. infantum to other areas.

Geographical distribution: (semi)arid areas - steppe and savannah in Asia (L. tropica, L. major, L. donovani), Africa (L. tropica, L. aethiopica, L. donovani) and the Mediterranean (L. tropica, L. infantum); forest ecosystems in Central and South America (L. mexicana, L. donovani, L. braziliensis, L. peruviana, L. chagasi). L. major and L. killicki occur in Tunisia. In 1999, an outbreak of canine leishmaniasis caused by L. infantum was reported in New York, and several other outbreaks followed in Foxhound kennels across the USA. For typical habitats of sandflies, see Photos 5.43 and 5.44 .

\subsubsection{Family Hexamitidae [Order Diplomonadida, Class Trepomonadea]}

\section{(**) Giardia lamblia (Synonyms G. intestinalis, G. duodenalis)}

The two life forms are an inactive ovoid cyst $(8-15 \times 7-10 \mu \mathrm{m})$ and active trophozoite - bilaterally symmetrical pyrifom-shaped flagellate 9-21×6-12 $\mu \mathrm{m}$ with four pairs of flagella, two nuclei and an adhesive disc. Several genomic types: A (anthroponotic), B (zoonotic), and C-G (animal types probably not transmissible to humans). The protozoan was first described by Lambl in 1859, as Cercomonas.

Source of infection (natural host range): human; less often rodents (castor, muskrat etc.), dog; rabbit, sheep; contaminated water (e.g., swimming pools). However, the importance of zoonotic transmission in human giardiosis is not clear.

Animal disease: inapparent course.

Transmission mode: alimentary (water contaminated with resistant cysts of rodents), by contact, mechanically via houseflies (Musca domestica) and cockroaches; high contagiosity. The cysts have considerable tenacity, and are resistant even to common disinfectants.

Human disease: giardiosis - intestinal form (giardiae adhere on intestine and duodenum mucosa): enteritis with abdominal pain, nausea, fatigue, 
diarrhoea, loss of weight and hepatobiliary damage. Extensive epidemics occurred in the 1970s (St. Petersburg, New York, Colorado). Giardiosis is a quite frequent human disease. For instance, the average annual incidence of giardiosis in Czechland was 645 in the period of 1990-2000, and Giardia was prevalent in about $1 \%$ adults and $4-5 \%$ children at the same time.

Bio-containment: BSL-2.

Diagnosis: microscopy of fresh stool using flotation method (4-nucleic cysts; flagellar trophozoites are usually present only in watery stool or in duodenal fluid), enteroscopy.

Treatment: albendazole, metronidazole, mepakrin, furazolidone; acridine dihydrochloride, paromomycin.

Geographical distribution: worldwide.

\subsubsection{Family Vahlkampfiidae [Order Schizopyrenida, Class Heterolobosea]}

\section{*Naegleria fowleri}

Amoeabe free-living in warm water, e.g. in indoor warmed swimming pools (the amoebae are able of replication in sand filters of the pool system). One isolate was recovered from a hot geyser in Yellowstone Park (USA), and a number of others from artesian wells in Arizona. They have two morphological forms: amoeba (a trophozoite) and a dormant cyst stage.

Source of infection: water (warm or warmed-up surface and well water).

Transmission mode: inhalatory - throw nasopharynx mucosa (amoeabe invade into brain along the olfactory nerves, and divide rapidly in the CNS); the cysts are extremely resistant in external milieu.

Human disease: primary amoebic meningoencephalitis (naegleriosis) - purulent, acute (3-10 days), causing haemorrhagic necrosis of the brain; the fatality rate is up to $100 \%$ even in young, immunocompetent persons. First epidemics have been observed in Australia in 1965 and also in Czechland in 1962-1965; in the latter country, 16 swimmers died, and other two fatal cases were recorded until 1984.

Bio-containment: BSL-2.

Diagnosis: microscopy of CSF under warm conditions (motility of trophozoites with a diameter of 7-20 $\mu \mathrm{m}$ ) or smear (IF), cultivation. Often misdiagnosed as a bacterial meningoencephalitis.

Treatment: amphotericin B (intravenously or intrathecally administered), in combination with fluconazole (or ketoconazole) and rifampicin.

Geographical distribution: probably worldwide, but only sporadic cases (Australia, New Zealand, USA, Czechland, Great Britain, Belgium). 


\subsubsection{Family Acanthamoebidae [Class Lobosea, Phyllum Rhizopoda]}

\section{*Acanthamoeba castellanii, A. polyphaga}

Amoebae (trophozoites $c .30 \mu \mathrm{m}$; cysts $10 \mu \mathrm{m}$ ) free-living in soil and water (cooling, waste, or contaminated seawater - these amoebae are frequently used as waste indicators in polluted water, sometimes they are more specific indicators of pollution than coliform bacteria or Clostridium perfringens). Interestingly, these protozoa may serve as obligatory hosts of legionellae and novel endosymbiont species Protochlamydia naegleriophila (isolated from immunocompromised patient with pneumonia).

Source of infection: water (thermal, polluted), soil.

Transmission mode: inhalatory (nasal mucosa, airways), per conjuctivae (contact lenses), even percutaneously (skin scratches). The cysts are very resistant to dessication, freeze and most disinfectants (including chlorination). Interestingly, Francisella tularensis survives in the cysts of A. castellanii for at least 3 weeks, and this amoeba can also harbour Legionella pneumophila for a long period or may serve as obligatory host of this bacterium.

Human disease: two main clinical forms - amoebic keratitis (usually in carriers of contact lenses), and chronic granulomatous amoebic meningoencephalitis (dissemination of the agent into CNS; most frequently in immunocompromised patients - e.g., with AIDS). Cutaneous lesions (hard erythematous nodules, papules or ulcers) caused by Acanthamoeba have also been described in immunocompromised persons.

Bio-containment: BSL-2.

Diagnosis: microscopy of CSF, scraping or rinsing slide prepartions from cornea (IF), confocal microscopy of corneal tissue, serology (IFA), cultivation (on 1.5\% non-nutrient agar plates with a growth of e.g. Escherichia coliamoebae feed on the bacteria, or on cell cultures - amoebae cause cytopathic effect), PCR.

Treatment: amphotericin B, sulphonamides, dibromopropamide (liniment), izothionate (eye drops), neomycin, itraconazole, miltefosine (alcylphosphocholin).

Geographical distribution: worldwide, ubiquitous.

\subsubsection{Family Leptomixidae [Class Lobosea]}

\section{*Balamuthia mandrillaris}

Large amoebae free-living in the soil, 50-60 $\mu \mathrm{m}$ in diameter, forming thick-walled cysts $15-30 \mu \mathrm{m}$. First isolated from the brain of a mandrill baboon Papio sphinx that died of encephalitis in San Diego Zoo, 1989. 
Source of infection: soil.

Transmission mode: inhalatory (nasal mucosa, airways), percutaneously (skin scratches), organ (kidney) transplantation (2 cases in USA); cysts are very resistant in external milieu.

Human disease: granulomatous amoebic encephalitis, a rare infection with lesions on skin and in nasopharynx, further hemipareses, ataxia, seizures, personality disturbances. A total of about 150 cases have been reported (half of them in the USA - predisposition was found in Hispanic and immunocompromised persons) since discovery of the disease in 1990, usually fatal. First case in Europe: Czechland, 1995.

Bio-containment: BSL-2.

Diagnosis: CT scan of the brain, microscopy of CSF, print (scraping, rinsing) of lesions (IF), cultivation, PCR; often post mortem.

Treatment: amphotericin B, sulphonamides, dibromopropamide (ointment), neomycin, itraconazole.

Geographical distribution: worldwide, sporadic.

\subsubsection{Family Thecamoebidae}

\section{* Sappinia pedata}

Two morphological forms as in other free-living amoebae (trophozoite and cyst stage).

Source of infection: soil contaminated with ruminant faeces.

Transmission mode: probably inhalatory.

Human disease: amoebic encephalitis, a rare infection (only one case has been described - in USA, 2001 - the patient was an immunocompetent and previously healthy man).

Bio-containment: BSL-2.

Diagnosis: as with other free-living amoebae.

Treatment: the patient survived after surgical excision of the tumour-like mass in the brain, and subsequent treatment with azithromycin, itraconazole, flucytosine and pentamidine.

Geographical distribution: worldwide.

\subsubsection{Family Eimeriidae [Order Eucoccidiida, Class Coccidea, Phyllum Apicomplexa]}

\section{*Cyclospora cayetanensis}

Source of infection: vegetables, herbs, strawberries, lettuce, red pepper, garlic, basil etc. 
Transmission mode: food-borne and water-borne.

Human disease: protracted and relapsing diarrhoea (incubation period about 7 days) in children and adults; often as a traveller diarrhoea. First cases were diagnosed in the late 1970s, and the first outbreak was reported from USA and Canada in 1995. Recently, a total of 29 cases were described in British Columbia (Canada) in 2007.

Bio-containment: BSL-2.

Diagnosis: microscopy of oocysts in stool samples (staining by Lugol's iodine, safranin, or Ziehl-Neelsen), PCR.

Treatment: cotrimoxazole (sulphamethoxazole + trimethoprim), pyrimethamine, ciprofloxacin.

Geographical distribution: probably worldwide, but predominantly in tropical and subtropical areas.

\subsubsection{Family Sarcocystidae [Order Eucoccidiida, Class Coccidea]}

\section{Sarcocystis bovihominis, S. suihominis (= S. miescheriana), S. lindemanni}

They have a two-host parasitic cycle: intermediate hosts are herbivores, with the tissue cysts (sarcocysts) containing thousands of cystozoites (merozoites, bradyzoites) in muscles; sexual stage of the cycle occurs in definitive (final) hosts - carnivores (gametogony generates oocysts with sporocysts and sporozoites). Humans and other primates can serve as definitive hosts for both $S$. bovihominis and S. suihominis.

Source of infection (natural host range): domestic animals (cattle $-S$. bovihominis, pig - suihominis).

Animal disease: whitish cysts in muscles, called Miescher's vesicles; in rodents so-called M-organism in the brain.

Transmission mode: alimentary (water or food contaminated faeces from unknown carnivore or omnivore in muscular form of infection; or raw or undercooked meat in intestinal form of infection).

Human disease: sarcosporidiosis (sarcocystosis) with two clinical forms: intestinal coccidiosis, when human serves as the definitive host ( $S$. bovihominis, S. suihominis), with nausea, anorexia, vomiting, abdominal pain, diarrhoea, tachycardia; and infrequent muscular coccidiosis (human serves as the intermediate host), with fever, musculoskeletal pain, rash, subcutaneous swelling, cardiomyopathy, occasional necrosis and atrophia of muscles, tongue, larynx, oesophagus, diaphragma, etc. (S. lindemanni).

Bio-containment: BSL-2.

Diagnosis: flotation of stool and microscopy (oocysts 20-33×10-16 $\mu \mathrm{m}$ with 2 sporocysts $15 \times 9 \mu \mathrm{m}$ each, containing 4 sporozoites) in intestinal form; biopsy in muscular form, serology (CFT). 
Treatment: cotrimoxazole, furazolidone, albendazole, sulphonamides (Biseptol) + pyrimethamine (but with unclear outcomes sometimes), anti-inflammatories; no specific treatment in muscle coccidiosis.

Geographical distribution: worldwide, endemic, with predominance in the tropical areas.

\section{Toxoplasma gondii}

Forms: (1) (acute stage) vegetative crescent-shaped trophozoites (endo- or tachyzoites) 4-7×2-4 $\mathrm{mm}$; in cell vacuoles often 2-32 together, forming "pseudocysts"; (2) (chronic stage) spherical tissue cysts 50-300 $\mu \mathrm{m}$ with thick membrane in muscles, heart, diaphragma and brain, with 1,000-60,000 crescent-shaped bradyzoites (cystozoites); (3) oocysts $(10-12 \mu \mathrm{m})$ discovered in the 1970s, result of sexual reproduction of gametocytes (gametogony) occurring only in the gut epithelium of definitive, final host. Sporulation (excitation) occurs only after excretion of immature oocysts, 2 sporocysts with 4 sporozoites $8 \times 2 \mu \mathrm{m}$ may be released from each oocyst. There are four genomic lineages of T. gondii.

Source of infection (natural host range): cat and other felids (Felis, Lynx) are definitive hosts of $T$. gondii (they become infected with meat of intermediate host such as rodents containing bradyzoites, and excrete oocysts in faeces during 1-3 weeks: about $1 \%$ of cats in Europe); intermediate hosts (only with asexual stages of parasite, cysts) are rodents (reservoir: TOT), lagomorphs (e.g., 80\% of domestic rabbits in Czechland have antibodies to T. gondii and $25 \%$ cysts), pig (cysts in 1-2\%, antibodies in 10\%), sheep (cysts in 5\%, antibodies in 40-60\%), cattle, and occasionally birds. In addition to the domestic cycle (domestic cat-mouse) there is a sylvatic cycle, observed e.g. in French Guyana: highly virulent strains of $T$. gondii circulate between free-living jaguars (Panthera onca, the definitive host) and their prey (intermediate hosts deer, armadillos, pacas, peccaries). Sources of human infection is undercooked meat from hunted game and water containing oocysts excreted by wild cats.

Animal disease: inapparent course, sometimes fever or even fatal infection (hen, pig, rabbit, hare in winter, rarely cat); abortions in sheep, goats, and pigs (Japan).

Transmission mode: alimentary (rare meat: bloody steaks or slightly smoked meat with tissue cysts, fruit, vegetables, water and soil contaminated with cat excrements containing oocysts, which are very resistant - they survive in cat faeces and water for up to 17 months); transplacentary. Among risk areas are children playgrounds with sand, which are visited by cats excreting the oocysts.

Human disease: toxoplasmosis - pantropic chronic disease: (1) congenital toxoplasmosis (infection of foetus $\rightarrow$ pathologic gravidity [acquiring infection during second trimester seems to be critical], miscarriage, chorioretinitis + 
hydrocephalus, microcephalia + CNS calcification of the foetus); (2) postnatal or acquired toxoplasmosis (lymphadenopathy, cough, fever, headache, fatigue, sometimes meningoencephalitis, chorioretinitis, various mental disorders), often latent (activation in AIDS patients etc.; 80-90\% of infection cases are asymptomatic). Toxoplasmosis is a common zoonosis in many countries. For instance, on average 1,008 (range, 670-2,049) clinical cases were reported annually in Czechland in the years 1990-2000, and about 30\% population revealed antibodies to $T$. gondii.

Diagnosis: serology (CFT, ELISA, Sabin-Feldman's test, which encompasses staining of trophozoites with alkaline methylen blue in the presence of examined sample and so-called activator, that is accesoric factor of native human serum; in the presence of antibodies have trophozoites characteristic original crescent-like shape with uncoloured plasma and blue nucleus, while without presence of antibodies they round off and stain deep blue; further serological tests include IFA, RDPA, RIHA etc.), intraperitoneal inoculation of mouse (and histology of its brain), biopsy of lymph nodes (IF, Giemsa: trophozoites have blue plasma, nucleus is red, plasmatic granula are red-brown); X-ray examination (calcification of the brain in newborns).

Bio-containment: BSL-2.

Treatment: sulphonamides for long-term usage, sulphadiazine (sulphamethoxydin) + pyrimethamine (Daraprim); antibiotics - spiramycin (during gravidity), clindamycin and doxycycline.

Prevention: necessary in pregnant women (avoid contact with raw or undercooked meat, cats). Vaccine unavailable.

Geographical distribution: worldwide (very often in tropics).

\subsubsection{Family Cryptosporidiidae [Order Eucoccidiida, Class Coccidea]}

\section{Cryptosporidium parvum, C. felis, C. canis, C. muris, C. suis, C. meleagridis}

Forms: oval trophocytes $2-4 \mu \mathrm{m}$ in the epithelial cells of respiratory and gastrointestinal tract of vertebrates including man. One-host developmental cycle (monoxenic), the host excretes oval thick-walled oocysts (sporocysts, with 4 sporozoites) about $5 \mu \mathrm{m}$.

Source of infection (natural host range): cattle (C. parvum bovine genotype II - a number of cases: e.g. major part of human isolates from Switzerland, also occurrence in Great Britain and Czechland), deer (C. parvum deer genotype), horse, sheep, cat (C. felis $>30$ cases), pig (C. parvum pig genotype), rodents (C. parvum, $C$. muris - sporadic cases), rabbit (C. parvum rabbit genotype), $\operatorname{dog}(C$. canis), birds ( $C$. meleagridis - several human cases); oysters and sea mussels; water - even after sand filtration. [Anthroponotic 
C. parvum genotype I, nowadays classified as a separate species $C$. hominis, is the principal causative agent of human cryptosporidiosis].

Animal disease: enteritis mainly in young animals (e.g., calves). Pathogenicity for animals was proven only in 1955, earlier the cryptosporidia were regarded as nonpathogenic, commensal species of many animals.

Transmission mode: alimentary (ingestion of oocysts excreted in faeces) mainly by water "water-borne" - largely the $C$. parvum bovine genotype 2 , but also food-borne; several epidemics reported after swimming in a pool (source: swimming pool filter backwash - 2000 Columbus, Ohio: 137 sick persons, 2000 Mallorca - a hotel swimming pool: 50 British tourists) or water parks. MID for man is only 30 oocysts, which are extremely resistant to chlorine (they survive at least $2 \mathrm{~h}$ in $5 \%$ of hypochlorite), they also survive in rotifers (Rotifera), and could be mechanically transmitted by house-flies. Many outbreaks of cryptosporidiosis occur in North America and Europe, and it is often unclear whether humans or animals are the source of contamination.

Human disease: cryptosporidiosis - enteritis lasting 1-2 weeks with fever, headache, abdominal pain, vomiting and diarrhoea (up to 20 times daily); relapses may occur. Disease was first described in 1976, it is common in immunocompromised persons e.g., with HIV infection $(C$. parvum, $C$. felis, C. canis, C. meleagridis, C. muris). A huge epidemic was recorded in Milwaukee (Wisconsin) in 1993: 403,000 persons were affected, 4,000 of them had to be hospitalised; the infection was spread by municipal water supply, which was contaminated by excrements of cattle.

Bio-containment: BSL-2.

Diagnosis: flotation of stool (in saturated solution of saccharose) and microscopy (oocysts), staining of stool specimens according to Miláček, or IF; serology (ELISA), PCR-RFLP.

Treatment: less effective - sulphonamides (trimethoprim + sulphomethoxazole, pyrimethamine), spiramycin, paromomycin; symptomatic treatment (Endiaron, Reasec).

Geographical distribution: worldwide.

\subsubsection{Family Plasmodiidae [Order Haemosporida, Class Haematozoea, Phyllum Apicomplexa]}

\section{${ }^{* *}$ Plasmodium falciparum, $P$. vivax, $P$. ovale, $P$. malariae}

The developmental cycle is complicated, it involves alteration of asexual replication (schizogony) and sexual development (sporogony). From a mosquito female, where sporogony takes place, sporozoites migrate into vertebrate blood during mosquito feeding, and in liver (cells of the RES) they change to cryptozoites (EE stadium, exoerythrocytic), and by schizogony into merozoites; the latter are released into blood and form trophozoites (ring-like forms) in erythrocytes; new merozoites arise

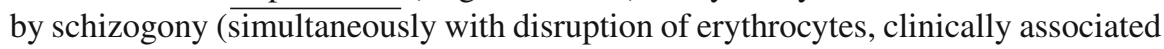


with fever), but also part of trophozoites remain in erythrocytes and change themselves on micro-(male) and macro-(female) gametocytes. Only these last stages could infect the vector mosquito again, where they transform in micro- and macrogametes, which form ookinete (zygota) after copulation that penetrates the mosquito midgut and produce oocysts in body cavity; sporozoites then form in the oocysts and migrate into salivary glands. Gametogony and sporogony occur thus exclusively in the mosquito vector.

Source of infection (natural host range): man (thus de facto anthroponosis).

Transmission mode: anopheline mosquitoes Anopheles maculipennis s.l. (south Europe - An. atroparvus, An. labranchiae), An. sacharovi (Asia Minor and Turkey), An. gambiae (Africa), An. darlingi and An. albimanus (South and Central America), group An. leucosphyrus and An. culicifacies (southeast Asia); iatrogenic (blood transfusion).

Human disease: malaria with several forms: tropica ( $P$. falciparum, the most serious - particularly in children and gravid women), tertiana ( $P$. vivax, $P$. ovale), and quartana ( $P$. malariae): period of schizogonic erythrocyte cycle is $48 \mathrm{~h}$ ("3-day malaria"), $72 \mathrm{~h}$ in P. malariae ("4-day malaria"); frequent relapses. Symptoms: chills, fever, hyperhidrosis, splenomegaly, sometimes affection of CNS (then often fatal course). WHO 1990: a total of 270 million people affected worldwide, approximately 1 million of which die annually. A total of about 20,000 cases of malaria are imported annually to Europe of them about 8,000 caused by $P$. falciparum. For example 918 cases in Germany in 1999 (60\% formed tourists travelling to endemic areas). Air carriage of infected mosquitoes in airplanes can result in "airport malaria" (infection of human via mosquito biting in the surroundings of airport or during intermediate landing; >100 cases have been registered since 1977, only in Paris 25 cases).

Bio-containment: BSL-2.

Diagnosis: microscopy - blood smear or thick drop (Giemsa), serology (IFA, RIHA, etc.). ELISA is a suitable serologic marker for detecting malaria in areas of low endemicity (lack of sensitivity of commonly used methods).

Treatment: chloroquine ( $P$. falciparum nowadays generally resistant; meflochine is an alternative), amodiachin, quinine + doxycycline, atebrin (uneffective on gametocytes); primaquine (only against parasites in liver and gametocytes); clindamycin, tetracycline.

Prophylaxis and prevention: proguanil, meflochine, chloroquine (resistance), pyrimethamine; repellents, and mosquito bed nets. Effective vaccine unavailable at present.

Geographical distribution: tropics and subtropics. Malaria has been extensively spreaded in Europe before WWR2 (commonly found in Mediterranean and Balkan) as well as in North America. Some hypotheses reveal that malaria could be one of the major causes of the fall of Roman Empire in fifth century. Italians controlled malaria in the neighbourhood of Rome by desiccation of lowland wetlands only in 1929. For some typical habitats of malaria, see Photos 5.45-5.48. 


\section{Plasmodium knowlesi, P. simium, P. cynomolgi}

Zoonotic species causing malaria in man.

Source of infection (natural host range): simian reservoir - monkeys (Aotus, Callithrix, Macaca fascicularis, M. nemestrina, Alouatta) and higher primates (chimpanzee, gorilla, orang-utan).

Animal disease: simian malaria.

Transmission mode: mosquitoes (Anopheles latens, An. cracens).

Human disease: simian malaria - previously regarded as a rare zoonosis, first described in 1965 ( $P$. knowlesi). Up to 2004, only 5 cases ( $P$. knowlesi) were reliably described. However, a number of additional cases have been reported since 2004 (at least 7, a few fatal) and it seems that simian malaria is an underdiagnosed (or misdiagnosed as classical human malaria), widely distributed disease in tropical countries.

Bio-containment: BSL-2.

Diagnosis: $P$. knowlesi is morphologically very similar to $P$. malariae and $P$. falciparum which could result in misidentification. Modern rapid diagnostic tests (e.g. OptiMAL-IT for detection of parasite lactate dehydrogenase, or even PCR) could produce cross-reactive or nonspecific results and miss P. knowlesi in the blood samples.

Treatment: identical to human malaria.

Geographical distribution: largely in forested areas of southeast Asia (Thailand, Malaysia, Sarawak, Borneo, Myanmar, Singapore, Vietnam, China - Yunnan province, the Philippines); rare in West Africa, Brazil.

\subsubsection{Family Babesiidae [Order Piroplasmida, Class Haematozoea, Phyllum Apicomplexa]}

\section{Babesia divergens, B. microti, B. duncani (WA1), B. venatorum (EU1)}

Very small $(1.5-2.0 \mu \mathrm{m})$ erythrocyte parasites of vertebrates (intermediate hosts) with final progression in ticks, their former designation piroplasmas has derived from latin "pirus" = pear, and it describes their characteristic shape in erythrocytes. According to up-to-date molecular studies (based on 18S rRNA gene sequencing) it has been shown that mentioned species are phylogenetically related more closely to the genus Theileria than to typical representatives of the genus Babesia, so we could expect some nomenclatural changes in foreseeable future.

Source of infection (natural host range): rodents of the genera Microtus and Peromyscus (B. microti), cattle (B. divergens), roe deer (B. microti, B. venatorum), and some other mammals.

Animal disease: piroplasmosis (babesiosis) with fever, haemoglobinuria, icterus, and haemolytic anaemia causing major losses in cattle (B. divergens, B. major, B. bigemina); haemolytic dog anaemia (B. canis). 
Transmission mode: ixodid ticks Ixodes scapularis (B. microti), I. ricinus ( $B$. microti, B. venatorum - TST and TOT confirmed, B. divergens, B. duncani), further Rhipicephalus, Dermacentor, Haemaphysalis, mainly by nymphs (inside them are present "vermicules" up to $16 \mu \mathrm{m}$ long); blood transfusion (a number of cases have been recorded in the USA).

Human disease: babesiosis - the course similar to malaria, with incubation period about 1-12 months: high fever (chronic in untreated patient) with chills, hyperhidrosis, exudation, fatigue, anorexia, headache, myalgia, cough, arthralgia, hepatosplenomegaly, icterus, haemolytic anaemia, thrombocytopenia, increased level of lactate dehydrogenase and bilirubin, haemoglobinuria, kidney failure. The most susceptible are splenectomized or otherwise immunocompromised people (B. divergens $-c$. 40 fatal cases in Europe: the former Yugoslavia, Scotland, Russia, France, Spain, Finland); B. microti is responsible for severe disease even in immunocompetent persons with spleen; fatality rate is about $5 \%$. Often longlife carriage of the parasite (blood of these persons can not be used for transfusion). Only few cases of the disease caused by $B$. venatorum have been recorded recently in Europe (Italy, Austria, Germany - all in splenectomized persons), and two symptomatic infections with B. microti (Switzerland - autochthonous, and Czechland - import from USA).

Bio-containment: BSL-2.

Diagnosis: blood smear, thick drop (Giemsa staining: oval or ring-like forms resembling trophozoites of Plasmodium falciparum in erythrocytes, sometimes in characteristic shape of Maltese cross); intraperitoneal inoculation of golden hamster; serology (IFA, ELISA; cross-reaction with Plasmodium), PCR of blood samples.

Treatment: a combination of quinine + clindamycin, the alternative is atovaquone + azithromycin; blood transfusion if necessary.

Geographical distribution: North America (except of B. divergens; B. duncani in west USA), Europe (except of $B$. duncani): Ireland, Scotland, France (B. venatorum), Switzerland, Italy, Austria, Germany (B. venatorum), Slovenia, Serbia, Russia, Czechland (evidence of B. microti in Ixodes ricinus ticks), Poland (B. venatorum), Finland (B. divergens), and China (B. microti). For a natural focus of babesiosis due to B. microti, see Photo 5.49 .

\subsubsection{Family Balantidiidae [Order Trichostomatida, Class Litostomatea, Phyllum Ciliophora]}

\section{Balantidium coli}

The largest pathogenic protozoan and only one medically important ciliate: it forms motile trophozoites 50-300×40-70 $\mu \mathrm{m}$, and spherical cysts 50-65 $\mu \mathrm{m}$.

Source of infection (natural host range): pig, wild boar (reservoir: a commensal of the swine intestine); occasionally rats, monkeys, dog. 
Animal disease: inapparent course.

Transmission mode: alimentary (cysts from pigs present in water).

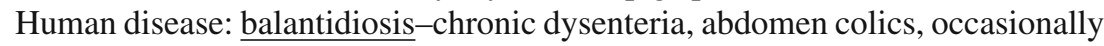
peritonitis.

Bio-containment: BSL-2.

Diagnosis: microscopy of stool (motile trophozoites, less often cysts), biopsy (scraping of intestinal mucosa), serology (IFA, immobilization).

Treatment: tetracycline, metronidazole, hydroxychinolin, sulphaguanidine.

Geographical distribution: worldwide (more frequently in tropics and subtropics), a sporadic occurrence.

\subsection{Other Eucaryotic Microorganisms}

\subsubsection{Algae}

\section{Family Chlorellaceae [Order Chlorellales, Class Chlorophyceae]}

\section{*Chlorella spp.}

Unicellular green spherical algae, reproducing asexually by internal septation, producing up to 20 "endospores" within the parent "sporangium".

Source of infection: river water.

Animal disease: unknown.

Transmission mode: by contact with traumatized skin.

Human disease: chlorellosis - a rare disease (only one case has been described up to now) with wound lesion draining a greenish-yellow exudate.

Bio-containment: BSL-2.

Diagnosis: microscopy of the tissue (endosporulating cells 6-9 $\mu \mathrm{m}$, with bright green granules), culture.

Treatment: surgical, amphotericin B.

Geographical distribution: USA (the human case).

\section{*Prototheca wickerhamii, P. zopfii}

Oval to spherical cells, morphologically similar to Chlorella, but without the green pigment.

Source of infection: grass, soil, water; animals.

Animal disease: unknown.

Transmission mode: by contact (traumatic inoculation).

Human disease: protothecosis - an uncommon disease (but more frequent than chlorellosis), with skin lesions (nodules, papules), occasionally disseminated 
(algaemia demonstrated) and then fatal (especially in immunocompromised patients).

Bio-containment: BSL-2.

Diagnosis: microscopy of the tissue (endosporulating hyaline cells 5-15 $\mu \mathrm{m}$ ), culture.

Treatment: surgical excision of localized lesions, antifungal agents (amphotericin B, itraconazole, fluconazole, ketoconazole).

Geographical distribution: worldwide.

\subsubsection{Blastocystea [Class Opalinata, Phyllum Chromista]}

\section{(**) Blastocystis hominis}

Formerly classified in Protozoa. A total of 9 genomic types have been differentiated, based on 18S rRNA analyses. Four morphologic forms (ontogenetic phases) are present: vacuolar form (2-200 $\mu \mathrm{m}$, with central vacuole); granular form (forming of granules in central vacuole); amoebic form (motile, with pseudopodia); thick-walled cyst (very resistant).

Source of infection (natural host range): human; less often pig, horse, cattle, brown rat, opossum, chickens (some animal genotypes have zoonotic potential - especially those from cattle and pig).

Animal disease: usually asymptomatic infection.

Transmission mode: alimentary (ingestion - water, food), tenacity of cysts is quite high - they resist even gastric fluid.

Human disease: blastocystosis - usually an acute gastrointestinal disease with diarrhoea, anorexia, abdominal pains and convulsions, sometimes with fever and fatigue.

Bio-containment: BSL-2.

Diagnosis: microscopy, serology (ELISA), PCR, cultivation is also possible.

Treatment: metronidazole.

Geographical distribution: worldwide, with majority in (sub)tropical countries.

\subsubsection{Microsporidia [Order Microsporida, Class Microsporea, Phyllum Microspora]}

Microsporidia, formerly classified as protozoans, are more related to fungi, but belong systematically probably to a separate class of microorganisms based on rRNA analyses. They are obligate intracellular parasites of invertebrates (especially arthropods) and vertebrates (mainly fishes) with ontogenic phases: schizogony (by another name merogony: binary fission in so-called parasitophoric vacuoles of host cells) and sporogony, followed by the release of spores from split host cell. Ovoid to pyriform small spores are very characteristic $(1.5-4.0 \times 1.0-3.0 \mu \mathrm{m})$ and contain 
one (Encephalitozoon, Enterocytozoon etc.) or two nuclei (binucleate), a posterior vacuole (polaroplast) and ejection apparatus (a tubulous fibre, which is in the caudal part of spores helically stranded into 4-8 coils and after swelling of the polaroplast ejectable throw frontal polar cap); sporoplasma with nucleus is dislodged into host cell after the fibre ejection. Microsporidia do not contain mitochondria, only their residua (called mitosomes). The microsporidian Nosema bombycis is the first discovered microbial parasite of insects, namely of silkworm Bombyx mori (Louis Pasteur studied this disease). Except for the species mentioned below, zoonotic transmission of other microsporidia to man has been demonstrated only rarely, especially in the genera Pleistophora, Tachipleistophora, Brachiola and Vittaforma.

\section{(**) Encephalitozoon cuniculi, E. intestinalis, E. hellem}

Source of infection (natural host range): human; rarely rabbit (E. cuniculi I), hare (E. intestinalis, E. hellem), rodents (E. cuniculi II), dog (E. cuniculi III), parrots (E. hellem), waterfowl (E. hellem, E. intestinalis).

Animal disease: usually asymptomatic infection. Sometimes clinical illness can be observed in rabbits, rodents (laboratory), carnivores (canine encephalitis-nephritis syndrome), and monkeys (usually in young animals).

Transmission mode: ingestion (surface water or groundwater, food) or inhalation, by contact, man-to-man transmission (male homosexuality). Tenacity of spores, which are excreted via urine and by expectorating, is quite high. The parasite can naturally persist in the human population and environment (even for several months under humid conditions).

Human disease: microsporidiosis (intestinal, respiratory and ocular form) chronic diarrhoea, frequently in immunocompromised persons (AIDS, organ transplantations), occasionally even necrosis of intestine, gallbladder, liver, kidney, pancreas, paranasal cavities and respiratory tract may evolve in these patients. Ocular microsporidiosis may occur in patients which were exposed to pet birds (oral or ocular autoinoculation throw contaminated fingers).

Bio-containment: BSL-2.

Diagnosis: light and transmission electron microscopy, IF and histochemical staining of samples (urine, stool and sputum), PCR, RFLP, sequencing; serology (IFA, ELISA: occasionally ambiguous outcomes).

Treatment: albendazole (only against E. intestinalis), metronidazole, cotrimoxazole, fumagilin.

Geographical distribution: worldwide.

\section{(**) Enterocytozoon bieneusi}

Very small spores, $1.5 \times 1.0 \mu \mathrm{m}$. A number of genotypes have been distinguished (A to $\mathrm{K}$ at present), and some of them are zoonotic. 
Source of infection (natural host range): man (the genotype B); zoonotic transmission of some genotypes to humans has been occasionally confirmed e.g., from cat, pig, dog, rabbit, rhesus monkey, cattle, goat, llama, wild mammals (beavers, foxes, muskrats, otters, raccoons), and recently also birds (chickens, pigeons).

Animal disease: usually asymptomatic infection.

Transmission mode: see Encephalitozoon.

Human disease: microsporidiosis - chronic diarrhoea and malabsorption syndrome mainly in persons suffering from AIDS (worlwide mean prevalence of E. bieneusi used to be $15-30 \%$ in those persons); less often in immunocompetent persons, usually as so-called traveller's diarrhoea. Mostly asymptomatic in humans: e.g., $10 \%$ of healthy persons have been found seropositive in Czechland.

Bio-containment: BSL-2.

Diagnosis and treatment: similar as for Encephalitozoon.

Geographical distribution: worldwide, sporadic.

\section{Brachiola (Nosema) algerae}

A microsporidian parasite of anopheline mosquitoes.

Source of infection (natural host range): probably infected mosquitoes and other insects.

Transmission mode: mosquito bites?

Human disease: 3 human cases have been described, also in immunocompetent persons: corneal lesions; a cutaneous nodule; lesions in muscle tissue.

Bio-containment: BSL-2.

Diagnosis and treatment: similar as for other microsporidia.

Geographical distribution: probably worldwide, but sporadic.

\subsubsection{Dermocystida [Class Mesomycetozoea, Phyllum Choanozoa]}

\section{${ }^{*}$ Rhinosporidium seeberi}

Systematic classification of this species was unclear for long times. However, it was found (based on 18S rRNA analyses) that rhinosporidia do not belong either to fungi, where were assigned formerly, or to typical protozoa; they are more closely related to animals. From phylogenetic point of view they are very close to the genera Dermocystidium and Amphibiocystidium, whose representatives are parasites of fishes and amphibians; corresponding order Dermocystida belongs to microbial Protista of the class Mesomycetozoea (earlier Ichthyosporea). 
Source of infection: stagnant or lacustrine water.

Animal disease: rhinosporidiosis of fishes, amphibians and rarely waterfowl. It has been also documented in many mammalian species, including cats, dogs, cattle, and horse (equine cases are infrequent but have been reported from the southern United States, South America, South Africa, and Great Britain).

Transmission mode: inhalation, ingestion, per conjunctivae from water (swimming), or percutaneous (injury).

Human disease: rhinosporidiosis - chronic granulomatous polyps of raspberry appearance in nasal mucosa, nasopharynx, and conjuctiva. Occupational risk: farmers working on rice fields.

Bio-containment: BSL-2.

Diagnosis: macro- and microscopy, biopsy, histology ( $R$. seeberi forms big thick-walled spherical sporangia in the affected tissue with diameter 50$1,000 \mu \mathrm{m}$ containing endospores $5-10 \mu \mathrm{m}$ ); cultivation impossible.

Treatment: surgical excision of the lesions, but recurrences are common.

Geographical distribution: tropical areas (Sri Lanka, India, southeast Asia, South America), rare autochthonous cases recorded also in Egypt, Turkey, Congo, Canada and USA (Florida), and Europe (UK). 